\title{
Innovative techniques for extending the range and node limits in Bluetooth based wireless sensor networks.
}

\author{
by \\ Matthew James Fraser \\ BEng. (Hons) Griffith University
}

Submitted in fulfilment of the requirements of the degree of Master of Philosophy 


\begin{abstract}
Wireless sensor networks for sports monitoring applications support data collection from multiple sensor platforms. Important network requirements are data throughput, range, low power operation and number of sensor nodes. The Bluetooth specification has been designed for low power, medium data rate, cable replacement solutions and as such is useful for wireless sensor networks. However it has a limitation of a maximum number of eight active devices per Bluetooth network (Piconet).

This project investigates and extends the capability of the Bluetooth specification and increases the usefulness for sensor applications. This has been achieved by utilising a combination of a network of Bluetooth wireless access points and innovative Bluetooth network structures. The Bluetooth network structures were created and controlled via the fixed Bluetooth access points, which act as root nodes for the network structures. The developed network structures for wireless sensing applications were Piconet Sharing, Scatternet Tree, Piconet Tree and Multiple Piconet. These network structures all allow more than seven Bluetooth devices to communicate to a fixed Bluetooth access point. A MATLAB based simulation tool was developed to compare the theoretical throughput per node for each network structure and validated experimentally.

Bluetooth hardware was used to test the performance of the proposed network structures with current Bluetooth hardware and software implementations. Several limitations were discovered with the test Bluetooth hardware used and this impacted on the performance of the Bluetooth network structures. All network structures were shown (via data extrapolation) to provide the throughput per node required for the specific wireless sensing application.

The developed network of Bluetooth root nodes combined with the developed network structures provided a complete solution suitable for wireless sensor applications. The same principles developed for Bluetooth based wireless sensor networks can be applied to other wireless applications that require a greater number of nodes than standard Bluetooth Piconets can provide. The developed network structures expand the applications that the Bluetooth Specification is suitable for.
\end{abstract}




\section{Acknowledgements}

There are many people to thank for assistance throughout this work and the completion of this thesis. Without the following people this project could not have been as successful as it was.

Thanks must go to my primary supervisor Dr Daniel James for the opportunity to do this project. Danny provided general guidance throughout the project and was especially helpful in constant reminders in writing my thesis. My secondary supervisor Professor David Thiel must also be thanked for his general project guidance and proof reading of this thesis.

Mr Neil Davey consistently provided technical help and moral support throughout the project. Neil provided invaluable Windows programming advice, help with embedded driver issues and was always available to bounce ideas off. Without Neil's assistance the project would not have been as successful as it was. Dr David Rowlands also provided valuable technical advice including help with driver issues, open source software and Unix based operating systems. Mr Leon Gourdeas provided valuable hardware advice throughout the project.

Simon Graham, Mark Shultze, Nathan Robinson, Rebecca Todd and Wayne Uroda must all be thanked for their help with on-field testing. Without their help the testing procedure would have taken much longer, resulting in many more hours in the hot sun.

Finally thanks must go to the CRC for MicroTechnology, The Center for Wireless Monitoring and Applications and the School of Microelectronic Engineering Griffith University for the opportunity to undertake this work. I owe a great debt of thanks for the support this team of people have shown me. 


\section{Statement of Originality}

This work has not previously been submitted for a degree or diploma in any university. To the best of my knowledge and belief, the thesis contains no material previously published or written by another person except where due reference is made in the thesis itself.

Signed:

Matthew James Fraser

Date: 


\section{Table of Contents}

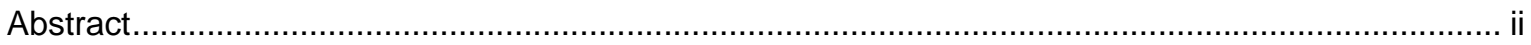

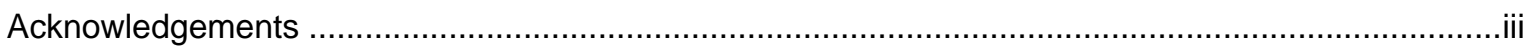

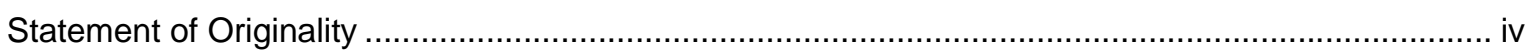

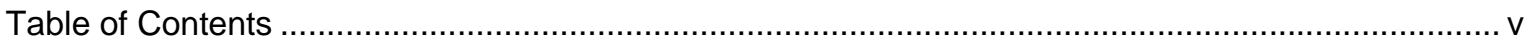

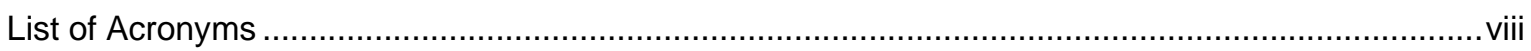

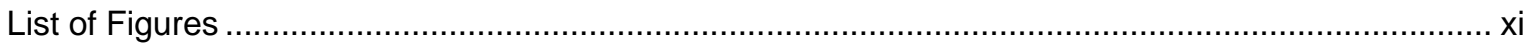

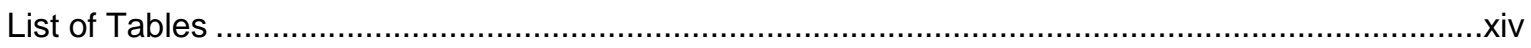

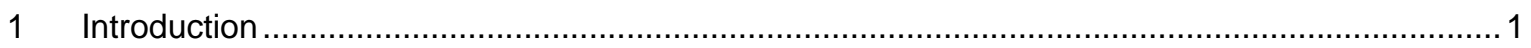

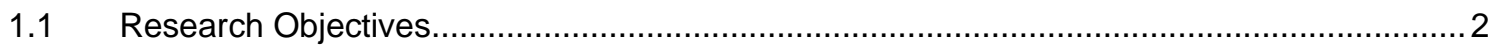

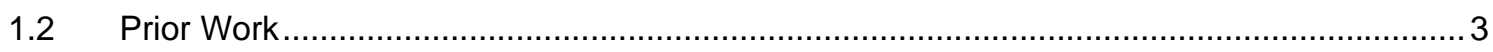

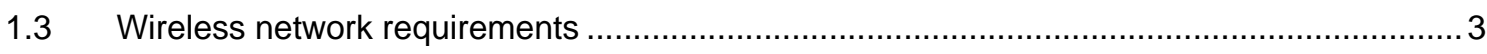

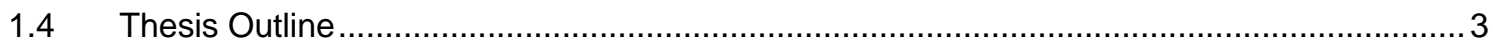

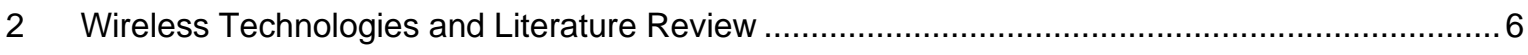

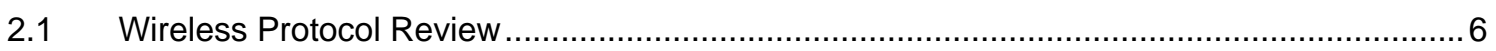

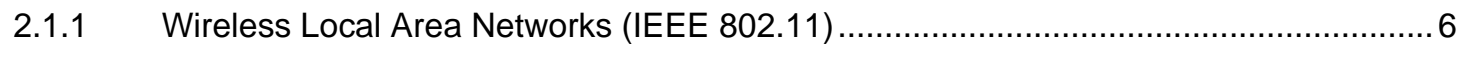

2.1.2 Wireless Personal Area Networks (IEEE 802.15) ................................................... 8

2.1.3 Wireless Metropolitan Area Networks (IEEE 802.16) .............................................. 13

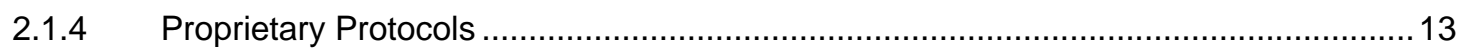

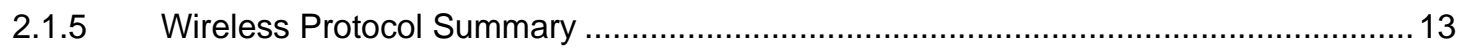

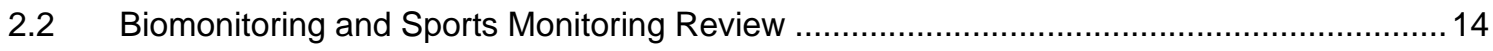

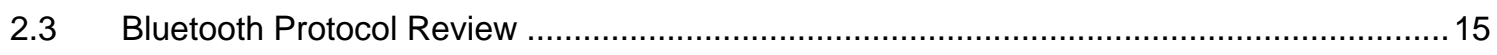

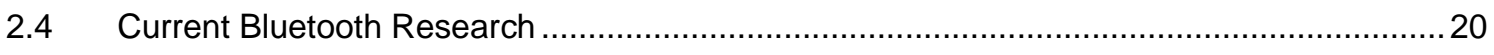

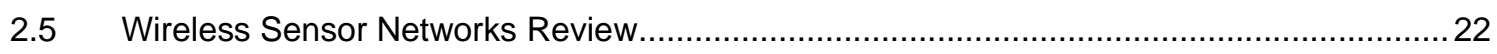

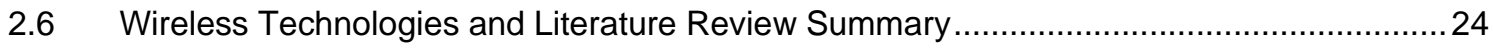

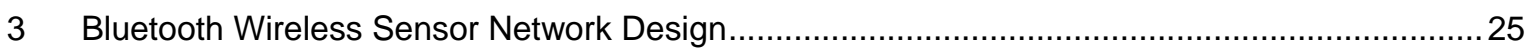

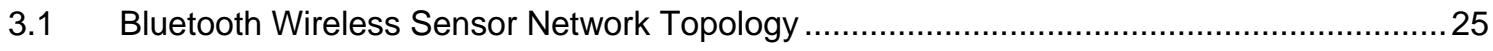

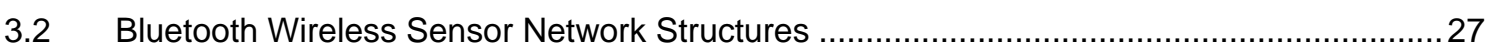

3.2.1 Standard Piconet Bluetooth Network Structure .................................................... 27

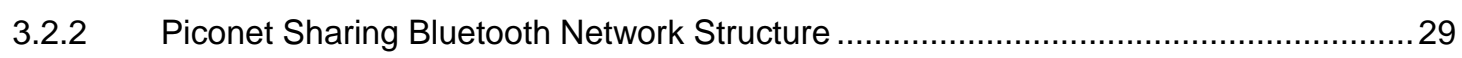

3.2.3 Scatternet Tree Bluetooth Network Structure ........................................................ 33

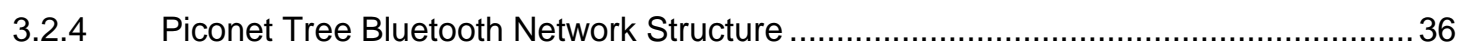

3.2.5 Multiple Piconet Bluetooth Network Structure ........................................................... 37

3.3 Bluetooth Wireless Sensor Network Design Summary ……............................................ 38

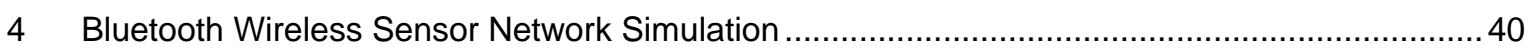




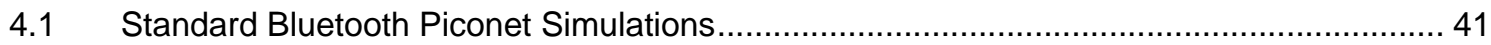

4.1.1 Standard Piconet Bluetooth Network Structure Simulation ................................... 41

4.1.2 Bluetooth Piconet Coexistence Simulation ................................................... 48

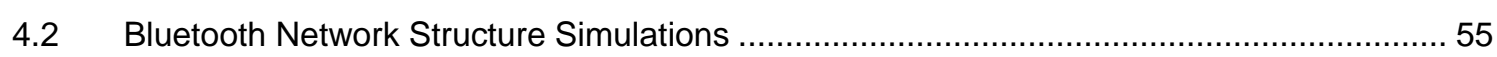

4.2.1 Piconet Sharing Bluetooth Network Structure Simulation.................................. 56

4.2.2 Scatternet Tree Bluetooth Network Structure Simulation .........................................6 66

4.2.3 Piconet Tree Bluetooth Network Structure Analysis ....................................... 76

4.2.4 Multiple Piconet Bluetooth Network Structure Analysis ......................................... 77

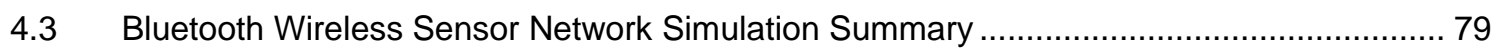

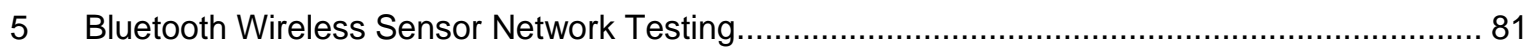

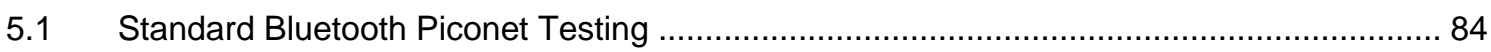

5.1.1 Bluetooth Wireless Sensor Node Testing .......................................................... 85

5.1.2 Root Node Created Standard Piconet Bluetooth Network Structure Testing .............. 90

5.1.3 Mobile Node Created Standard Piconet Bluetooth Network Structure Testing ........... 92

5.2 Developed Bluetooth Network Structure Testing ....................................................... 93

5.2.1 Piconet Sharing Bluetooth Network Structure Testing ...................................... 93

5.2.2 Scatternet Tree Bluetooth Network Structure Testing .......................................... 100

5.2.3 Piconet Tree Bluetooth Network Structure Testing .......................................... 105

5.2.4 Multiple Piconet Bluetooth Network Structure Testing .......................................... 109

5.3 Bluetooth Wireless Sensor Network Testing Summary ........................................... 114

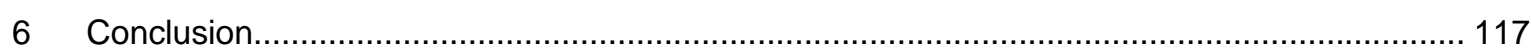

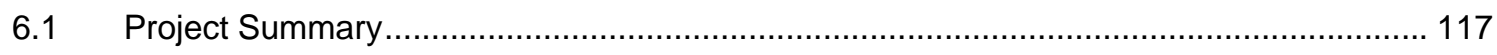

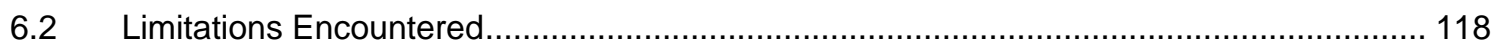

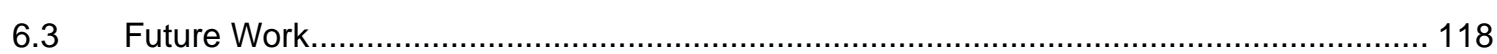

6.4 Bluetooth Wireless Sensor Network Recommendations .......................................... 119

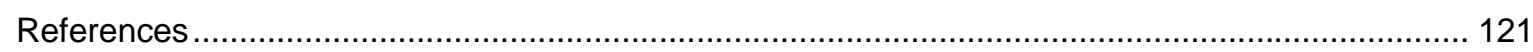

Appendix A: Standard Piconet Bluetooth Network Structure Simulation Code .......................... 127

Appendix B: Bluetooth Piconet Coexistence Simulation Code ............................................ 132

Appendix C: Piconet Sharing Bluetooth Network Structure Simulation Code .......................... 138

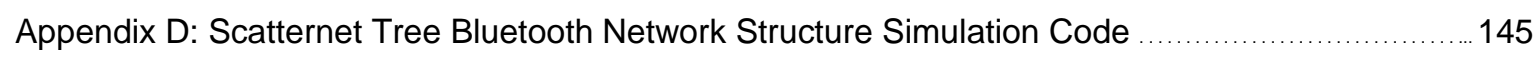

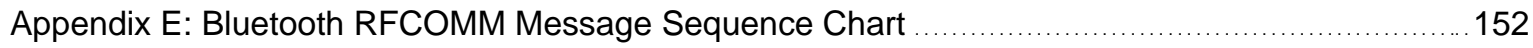

Appendix F: Bluetooth Piconet Sharing Message Sequence Chart .................................... 153

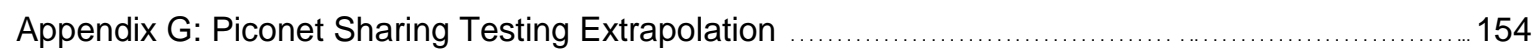

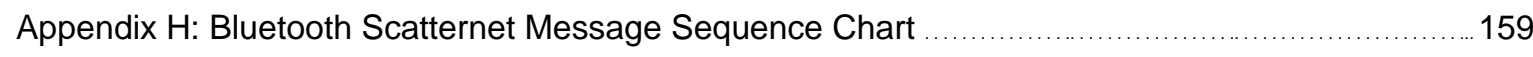

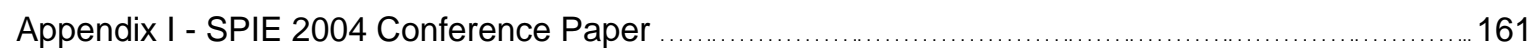


Appendix J: SPIE 2005 Conference Paper

Appendix K: The Japan Society of Mechanical Engineers Conference Paper

190 


\section{List of Acronyms}

This document contains many acronyms. For ease of reading the most commonly used acronyms have been tabulated as Table i, Table ii and Table iii.

\begin{tabular}{|l|l|}
\hline Acronym & Definition \\
\hline PAN & Personal area network \\
\hline LAN & Local Area Network \\
\hline WAN & Wide Area Network \\
\hline WLAN & Wireless Local Area Network \\
\hline WPAN & Wireless Personal Area Network \\
\hline IEEE & Institute of Electrical and Electronics Engineers \\
\hline ECMA & European Computer Manufacturers Association \\
\hline FHSS & Frequency Hopped Spread Spectrum \\
\hline DSSS & Direct Sequence Spread Spectrum \\
\hline MAC & Media Access Controller \\
\hline CSMA/CA & Carrier Sense Multiple Access Collision Avoidance \\
\hline TDMA & Time Division Multiple Access \\
\hline TDD & Time Division Duplex \\
\hline RTS & Request to Send \\
\hline CTS & Clear to Send \\
\hline OSI & Open Systems Interconnect \\
\hline CCK & Complimentary Code Keying \\
\hline BPSK & Bi-polar Phase Shift Keying \\
\hline QPSK & Quadrature Phase Shift Keying \\
\hline QAM & Quadrature Amplitude Modulation \\
\hline OFDM & Orthogonal Frequency Division Multiplexing \\
\hline SIG & Special Interest Group \\
\hline CSR & Cambridge Silicon Radio \\
\hline DEVs & IEEE 802.15.3 Standard Devices \\
\hline PNC & IEEE 802.15.3 Piconet coordinator \\
\hline UWB & Ultra-WideBand (UWB) \\
\hline & \\
\hline
\end{tabular}

Table i: List of Acronyms 


\begin{tabular}{|l|l|}
\hline Acronym & Definition \\
\hline MB-OFDM & Multi Band Orthogonal Frequency Division Multiplexing \\
\hline DS-UWB & Direct Sequence Ultra-WideBand \\
\hline USB & Universal Serial Bus \\
\hline WUSB & Wireless Universal Serial Bus \\
\hline EIRP & Effective Isotropically Radiated Power \\
\hline O-QPSK & Offset Quadrature Phase Shift Keying \\
\hline RFD & IEEE 802.15.4 Reduced Function Devices \\
\hline FFD & IEEE 802.15.4 Full Function Devices \\
\hline NWK & ZigBee Network Layer \\
\hline APL & ZigBee application layer \\
\hline IP & Internet Protocol (IP) \\
\hline WAP & Wireless Access Protocol (WAP) \\
\hline GPS & Global Positioning System (GPS) \\
\hline POLL & Bluetooth poll packet (POLL) \\
\hline EDR & Bluetooth Extended Data Rate (EDR) \\
\hline DMx & Bluetooth Data Medium Packets of length x (DMx) \\
\hline DHx & Bluetooth Data High Packets of length x (DHx) \\
\hline RSSI & receive strength signal indicator (RSSI) \\
\hline ISM Band & Industrial Scientific and Medical Frequency Band (ISM band) \\
\hline BD_ADDR & Unique Bluetooth Address (BD_ADDR) \\
\hline AM_ADDR & Bluetooth Active Member Address (AM_ADDR) \\
\hline PM_ADDR & Bluetooth Parked Member Address \\
\hline AR_ADDR & Bluetooth Access Request Address \\
\hline AFH & Bluetooth Adaptive Frequency Hopping \\
\hline PSK & Phase Shift Keying \\
\hline RF & Radio Frequency \\
\hline HCI & Host Controller Interface \\
\hline LMP & Bluetooth Link Manager Protocol \\
\hline L2CAP & Bluetooth Logical Link Control and Adaption Protocol \\
\hline SDP & Bluetooth Service Discovery Protocol \\
\hline SPP & Bluetooth Serial Port Profile \\
\hline
\end{tabular}

Table ii: List of Acronyms 


\begin{tabular}{|l|l|}
\hline Acronym & Definition \\
\hline APR & Bluetooth Access Point Roaming (APR) \\
\hline M & Bluetooth Piconet Master Node(M) \\
\hline S & Bluetooth Piconet Slave Node (S) \\
\hline M/S & Bluetooth Piconet Master and Slave Node (M/S) \\
\hline ACL & Bluetooth Asynchronous Connection-Less Connection (ACL) \\
\hline SCO & Bluetooth Synchronous Connection-Oriented Connection (SCO) \\
\hline SBC & Single Board Computer (SBC) \\
\hline WinCE & Windows Compact Edition (WinCE) \\
\hline BC02 & Cambridge Silicon Radio BlueCore02 Bluetooth Radio (BC02) \\
\hline
\end{tabular}

Table iii: List of Acronyms 


\section{List of Figures}

Figure 1: A Typical Wireless Sensor Network

Figure 2: Bluetooth network structures; (a) single Piconet structure, (b) master / slave bridging node

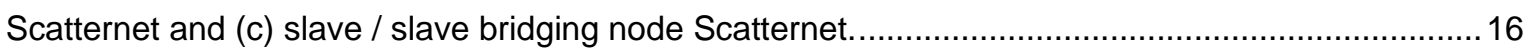

Figure 3: Bluetooth theoretical baseband and quoted RFCOMM throughput per slave $[1,29] \ldots \ldots \ldots . .17$

Figure 4: Bluetooth Stack as outlined by the Bluetooth Specification [1] ........................................ 19

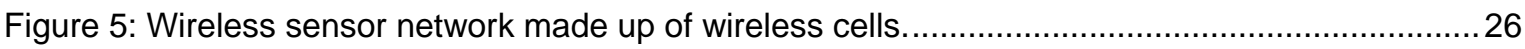

Figure 6: Standard Bluetooth Piconets; (a) Point to Point Connection and (b) Point to Multi-Point

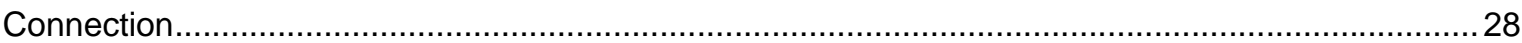

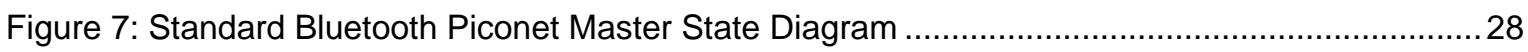

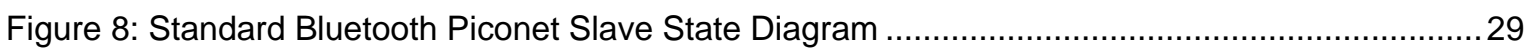

Figure 9: Piconet Sharing Bluetooth Network Structure; (a) Group 1 is Active and Group 2 is Parked

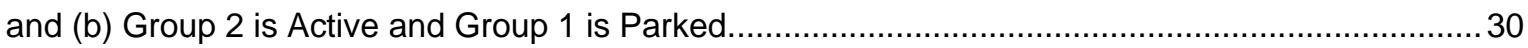

Figure 10: Bluetooth Piconet Sharing Master State Diagram ......................................................... 31

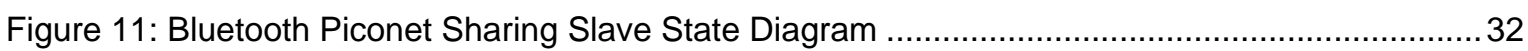

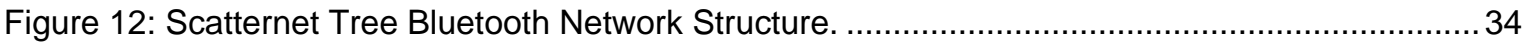

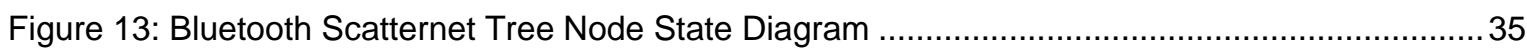

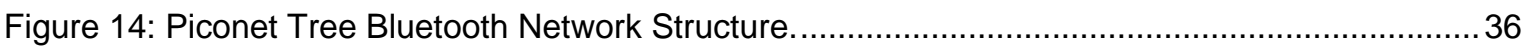

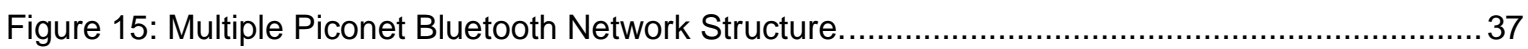

Figure 16: Example of Channel Access on a Bluetooth Piconet with Two Active Slaves..................... 42

Figure 17: Standard Bluetooth Piconet Network Structure Simulation Main Flow Diagram ................44

Figure 18: Standard Bluetooth Piconet Network Structure Simulation Flow Diagram ...........................45

Figure 19: Sample Output for Standard Bluetooth Piconet Simulation ............................................. 46

Figure 20: Example Standard Piconet Simulation Throughput per Slave Output ...............................47

Figure 21: Standard Bluetooth Piconet Simulation Network Throughput Results............................... 48

Figure 22: Bluetooth Piconet Coexistence Simulation Main Flow Diagram ....................................... 51

Figure 23: Bluetooth Piconet Coexistence Simulation Flow Diagram ............................................. 52

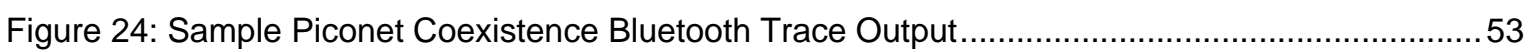

Figure 25: Sample Piconet Coexistence Simulation Throughput per Slave Output..............................54

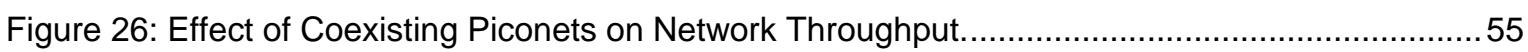

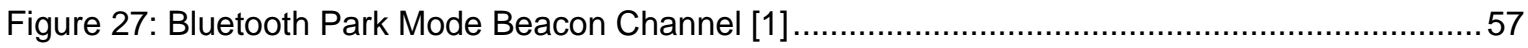

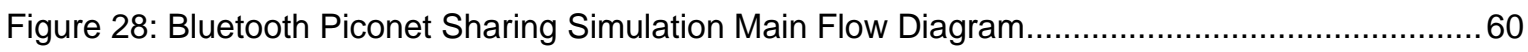

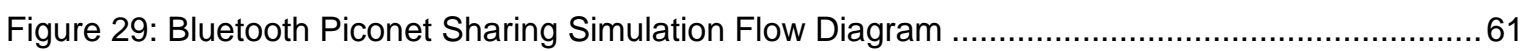

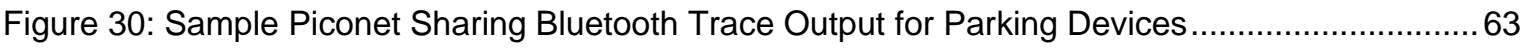

Figure 31: Sample Piconet Sharing Bluetooth Trace Output for Park Beacon ..................................... 63 
Figure 32: Sample Piconet Sharing Bluetooth Trace Output for Unparking Devices with Parking Beacon 64

Figure 33: Sample Piconet Sharing Simulation Throughput per Slave Output.

Figure 34: Average Throughput Results for Bluetooth Piconet Sharing Simulation for Differing

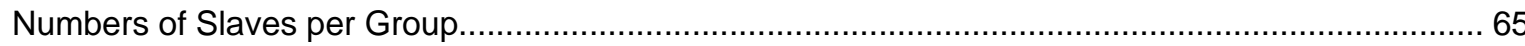

Figure 35: Average Throughput Results for Bluetooth Piconet Sharing Simulation ............................. 66

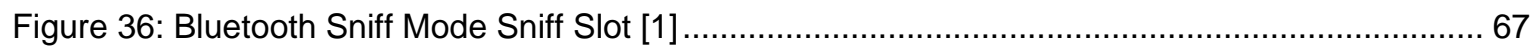

Figure 37: Bluetooth Scatternet Tree Structure Simulation Main Flow Diagram .................................. 69

Figure 38: Bluetooth Scatternet Tree Structure Simulation Flow Diagram...................................... 70

Figure 39: Example Scatternet Tree Bluetooth Trace Output.................................................... 72

Figure 40: Example Bluetooth Scatternet Tree Structure with Optimum Sniff Timeout; (a) Throughput per Node Output and (b) Master / Slave Node Data Buffer .............................................................. 73

Figure 41: Example Bluetooth Scatternet Tree Structure with Too Large Sniff Timeout; (a)

Throughput per Node Output and (b) Master / Slave Node Data Buffer ........................................ 73

Figure 42: Example Bluetooth Scatternet Tree Structure with Too Small Sniff Timeout; (a)

Throughput per Node Output and (b) Master / Slave Node Data Buffer ........................................ 74

Figure 43: Average Throughput Results for Bluetooth Scatternet Tree Structure Simulation.............. 75

Figure 44: Throughput Results for Bluetooth Piconet Tree Network Structure Simulation ................. 77

Figure 45: Throughput Results for Multiple Piconet Network Structure Simulation............................. 78

Figure 46: Bluetooth Hardware Used in Wireless Sensor Testing; (a) Bluetooth Root Node [64] and

(b) Wireless Sensor Node (Radio Only) [65]

Figure 47: Bluetooth Root Node and Wireless Sensor Node Stack Architecture (a) Single Board

Computer and Bluetooth Module ( $\mathrm{HCl}$ Firmware) and (b) Sensor Platform and Bluetooth Module (RFCOMM Firmware)

Figure 48: Bluetooth Device Connection and Reconnection Testing ............................................ 87

Figure 49: Bluetooth Unparking Times for Varying Park Beacon Lengths .......................................... 88

Figure 50: Throughput Drop Off with Increases Communication Range ......................................... 90

Figure 51: Measured Throughput per Slave for Root Node Created Standard Piconet...................... 91

Figure 52: Measured Throughput per Slave for Bluetooth Module Created Standard Piconet ........... 92

Figure 53: Piconet Sharing Node Extrapolation (a) Extrapolated Piconet Sharing Structure and (b)

Actual Tested Piconet Sharing Structure 94

Figure 54: Extrapolated Bluetooth Piconet Sharing Network Structure Minimum Throughput............ 98

Figure 55: Effect of Average Piconet Sharing Throughput of Piconet Sharing Group Active Time..... 99

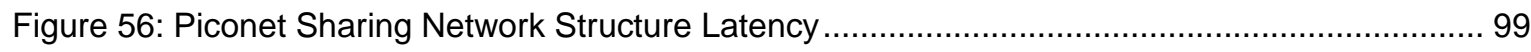

Figure 57: Extrapolated Bluetooth Scatternet Tree Network Structure Minimum Throughput ........... 104 
Figure 58: Bluetooth Piconet Tree Stack Architecture (a) Piconet Tree Bridging Node Stack Architecture and (b) Piconet Tree Sensing Node Stack Architecture 105

Figure 59: Extrapolated Bluetooth Piconet Tree Network Structure Minimum Throughput ............... 109

Figure 60: Bluetooth Multiple Piconet Network Structure Stack Architecture ................................. 110

Figure 61: Extrapolated Bluetooth Multiple Piconet Network Structure Minimum Throughput ...........113

Figure 62: Proposed Advanced Host Control Interface Bluetooth Stack Layer .................................. 114 


\section{List of Tables}

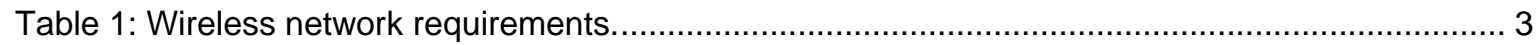

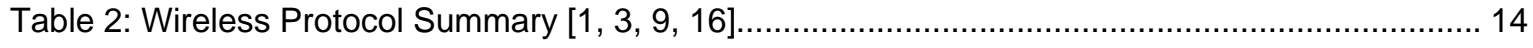

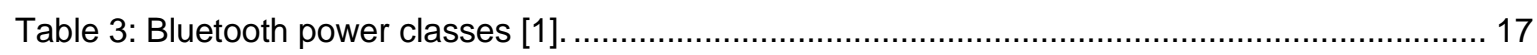

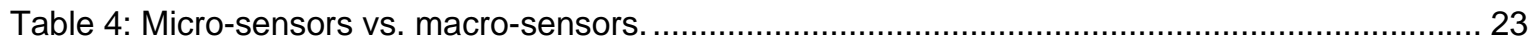

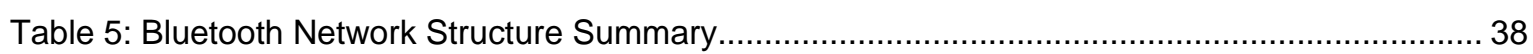

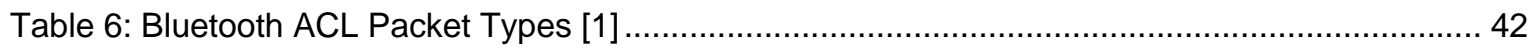

Table 7: Standard Bluetooth Piconet Network Structure Simulation Parameters................................ 45

Table 8: Comparison Between Simulation Results and Bluetooth Specification ................................. 47

Table 9: Bluetooth Piconet Coexistence Simulation Parameters ....................................................... 52

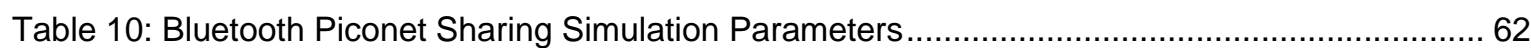

Table 11: Bluetooth Scatternet Tree Simulation Parameters ……............................................. 71

Table 12: Bluetooth Scatternet Tree Network Structure Possible Node Combinations ........................ 75

Table 13: Bluetooth Piconet Tree Network Structure Possible Node Combinations ............................. 76

Table 14: Bluetooth Multiple Piconet Network Structure Possible Node Combinations ....................... 78

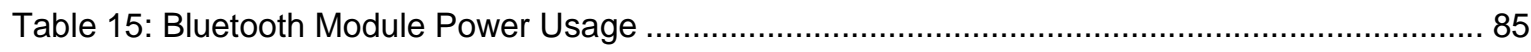

Table 16: Bluetooth Module Power Usage in Bluetooth Low Power States ......................................... 86

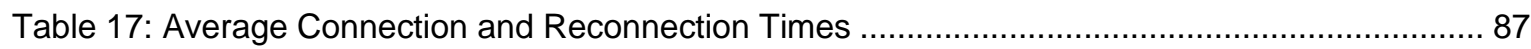

Table 18: Average Unparking Times for Varying Park Beacon Lengths ............................................ 89

Table 19: Extrapolated Piconet Sharing Hardware Testing Results............................................... 95

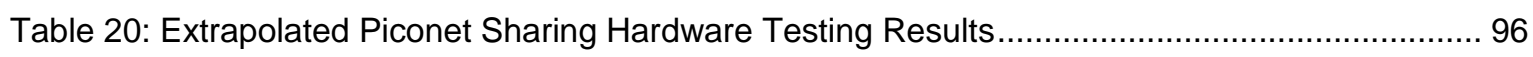

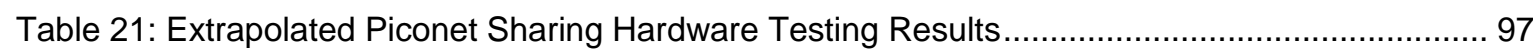

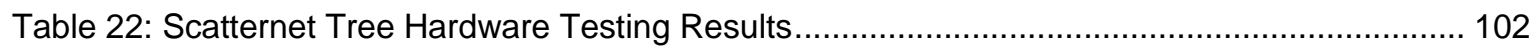

Table 23: Extrapolated Scatternet Tree Hardware Testing Results .............................................. 103

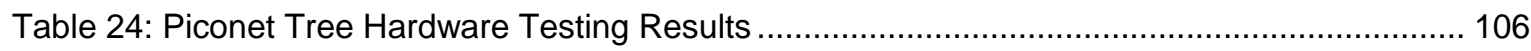

Table 25: Extrapolated Piconet Tree Hardware Testing Results.................................................... 107

Table 26: Extrapolated Piconet Tree Hardware Testing Results................................................ 108

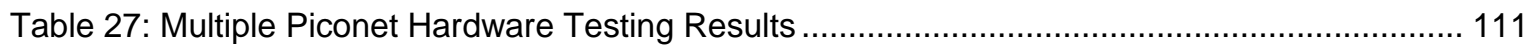

Table 28: Extrapolated Multiple Piconet Hardware Testing Results.............................................. 112 


\title{
Innovative techniques for extending the range and node limits in Bluetooth based wireless sensor networks.
}

\author{
by \\ Matthew James Fraser \\ BEng. (Hons) Griffith University
}

Submitted in fulfilment of the requirements of the degree of Master of Philosophy 


\section{Removal Notice}

Appendices I, $\mathrm{J}$ and $\mathrm{K}$ have been removed from the digital version of this thesis for copyright reasons.

Citations:

Appendix I

- M. J. Fraser, D. A. James and D. V. Thiel, Wireless Network Topology for

Monitoring Mobile Agents, Proceedings of SPIE vol 5651 Biomedical Applications of

Micro- and Nanoengineering II, Sydney, NSW, 2004

Appendix J

- M. J. Fraser, D. A. James and D. V. Thiel, Innovative techniques for extending the range and node limits in Bluetooth-based wireless sensor networks, Proceedings of SPIE vol 6035 Microelectronics: Design, Technology and Packaging II, Brisbane, QLD, 2005

Appendix K

- D. A. James and M.J. Fraser, The application of wireless technology and sensors for sporting applications, The Japan Society of Mechanical Engineers (Bioengineering Division), Sports Engineering Symposium, Kanazawa, 2006 


\begin{abstract}
Wireless sensor networks for sports monitoring applications support data collection from multiple sensor platforms. Important network requirements are data throughput, range, low power operation and number of sensor nodes. The Bluetooth specification has been designed for low power, medium data rate, cable replacement solutions and as such is useful for wireless sensor networks. However it has a limitation of a maximum number of eight active devices per Bluetooth network (Piconet).

This project investigates and extends the capability of the Bluetooth specification and increases the usefulness for sensor applications. This has been achieved by utilising a combination of a network of Bluetooth wireless access points and innovative Bluetooth network structures. The Bluetooth network structures were created and controlled via the fixed Bluetooth access points, which act as root nodes for the network structures. The developed network structures for wireless sensing applications were Piconet Sharing, Scatternet Tree, Piconet Tree and Multiple Piconet. These network structures all allow more than seven Bluetooth devices to communicate to a fixed Bluetooth access point. A MATLAB based simulation tool was developed to compare the theoretical throughput per node for each network structure and validated experimentally.

Bluetooth hardware was used to test the performance of the proposed network structures with current Bluetooth hardware and software implementations. Several limitations were discovered with the test Bluetooth hardware used and this impacted on the performance of the Bluetooth network structures. All network structures were shown (via data extrapolation) to provide the throughput per node required for the specific wireless sensing application.

The developed network of Bluetooth root nodes combined with the developed network structures provided a complete solution suitable for wireless sensor applications. The same principles developed for Bluetooth based wireless sensor networks can be applied to other wireless applications that require a greater number of nodes than standard Bluetooth Piconets can provide. The developed network structures expand the applications that the Bluetooth Specification is suitable for.
\end{abstract}




\section{Acknowledgements}

There are many people to thank for assistance throughout this work and the completion of this thesis. Without the following people this project could not have been as successful as it was.

Thanks must go to my primary supervisor Dr Daniel James for the opportunity to do this project. Danny provided general guidance throughout the project and was especially helpful in constant reminders in writing my thesis. My secondary supervisor Professor David Thiel must also be thanked for his general project guidance and proof reading of this thesis.

Mr Neil Davey consistently provided technical help and moral support throughout the project. Neil provided invaluable Windows programming advice, help with embedded driver issues and was always available to bounce ideas off. Without Neil's assistance the project would not have been as successful as it was. Dr David Rowlands also provided valuable technical advice including help with driver issues, open source software and Unix based operating systems. Mr Leon Gourdeas provided valuable hardware advice throughout the project.

Simon Graham, Mark Shultze, Nathan Robinson, Rebecca Todd and Wayne Uroda must all be thanked for their help with on-field testing. Without their help the testing procedure would have taken much longer, resulting in many more hours in the hot sun.

Finally thanks must go to the CRC for MicroTechnology, The Center for Wireless Monitoring and Applications and the School of Microelectronic Engineering Griffith University for the opportunity to undertake this work. I owe a great debt of thanks for the support this team of people have shown me. 


\section{Statement of Originality}

This work has not previously been submitted for a degree or diploma in any university. To the best of my knowledge and belief, the thesis contains no material previously published or written by another person except where due reference is made in the thesis itself.

Signed:

Matthew James Fraser

Date: 


\section{Table of Contents}

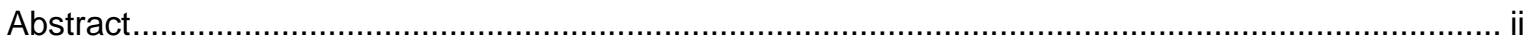

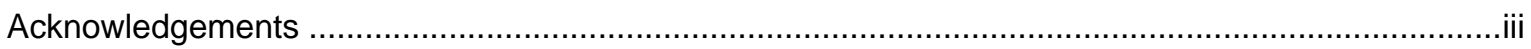

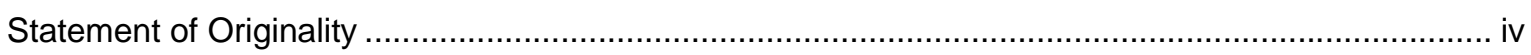

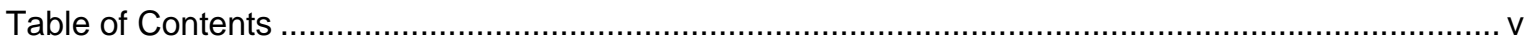

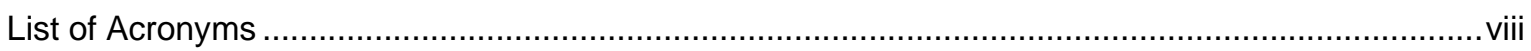

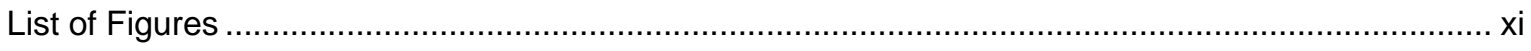

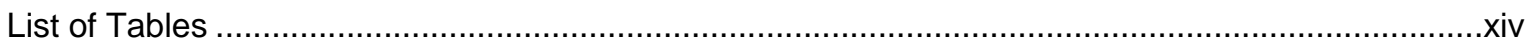

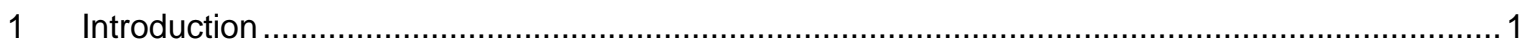

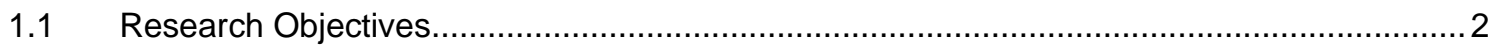

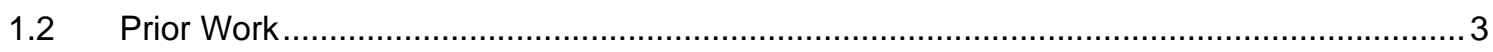

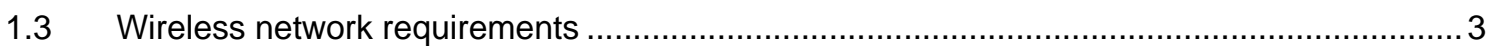

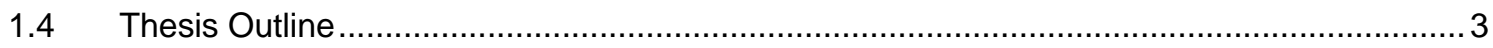

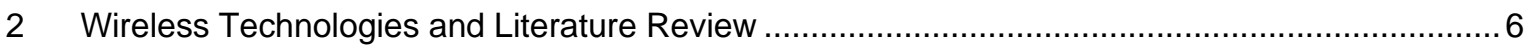

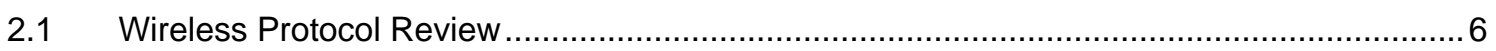

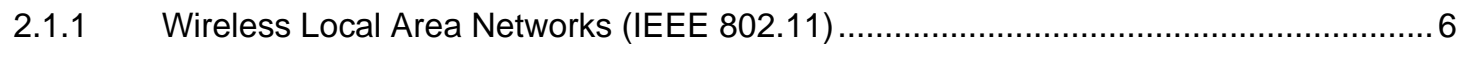

2.1.2 Wireless Personal Area Networks (IEEE 802.15) ................................................... 8

2.1.3 Wireless Metropolitan Area Networks (IEEE 802.16) .............................................. 13

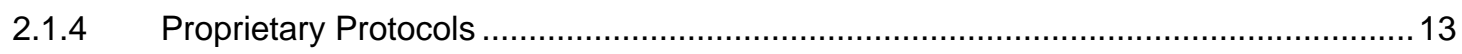

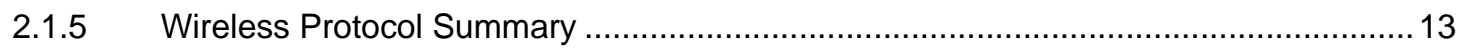

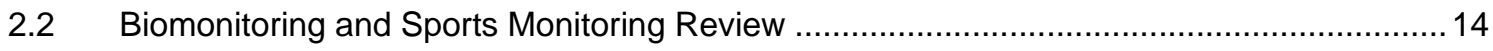

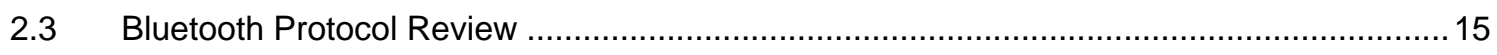

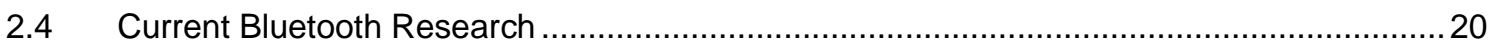

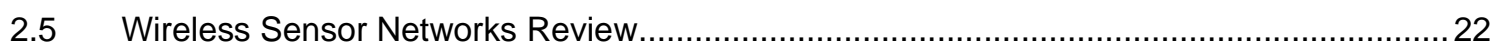

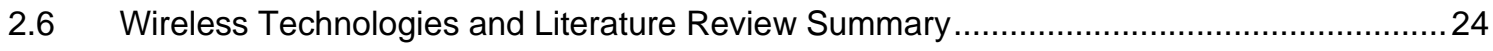

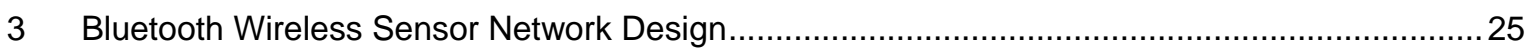

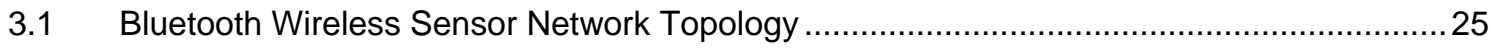

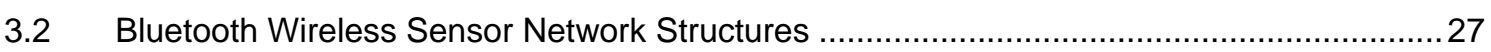

3.2.1 Standard Piconet Bluetooth Network Structure .................................................... 27

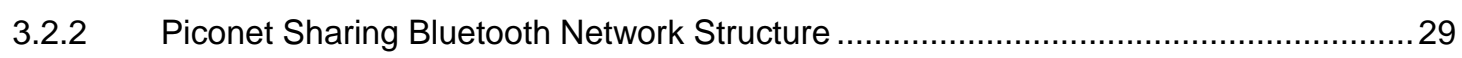

3.2.3 Scatternet Tree Bluetooth Network Structure ........................................................ 33

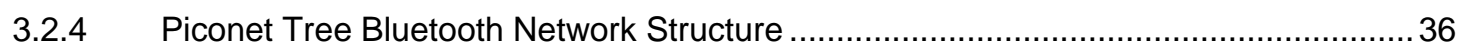

3.2.5 Multiple Piconet Bluetooth Network Structure ........................................................... 37

3.3 Bluetooth Wireless Sensor Network Design Summary ……............................................ 38

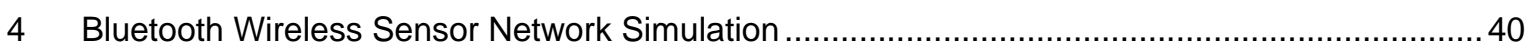




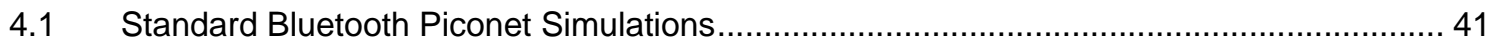

4.1.1 Standard Piconet Bluetooth Network Structure Simulation ................................... 41

4.1.2 Bluetooth Piconet Coexistence Simulation ................................................... 48

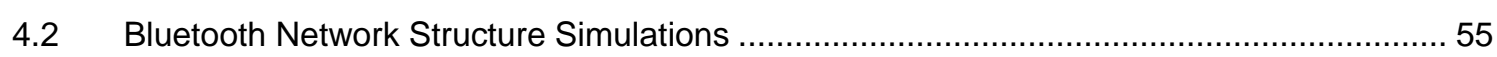

4.2.1 Piconet Sharing Bluetooth Network Structure Simulation.................................. 56

4.2.2 Scatternet Tree Bluetooth Network Structure Simulation .........................................6 66

4.2.3 Piconet Tree Bluetooth Network Structure Analysis ....................................... 76

4.2.4 Multiple Piconet Bluetooth Network Structure Analysis ......................................... 77

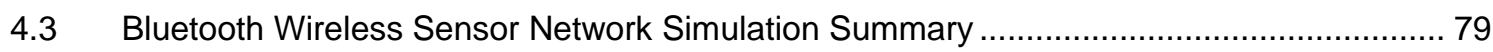

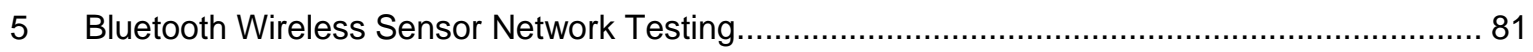

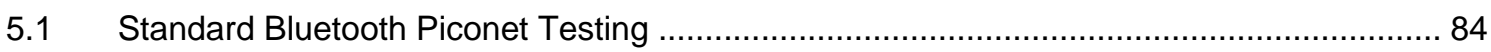

5.1.1 Bluetooth Wireless Sensor Node Testing .......................................................... 85

5.1.2 Root Node Created Standard Piconet Bluetooth Network Structure Testing .............. 90

5.1.3 Mobile Node Created Standard Piconet Bluetooth Network Structure Testing ........... 92

5.2 Developed Bluetooth Network Structure Testing ....................................................... 93

5.2.1 Piconet Sharing Bluetooth Network Structure Testing ...................................... 93

5.2.2 Scatternet Tree Bluetooth Network Structure Testing .......................................... 100

5.2.3 Piconet Tree Bluetooth Network Structure Testing .......................................... 105

5.2.4 Multiple Piconet Bluetooth Network Structure Testing .......................................... 109

5.3 Bluetooth Wireless Sensor Network Testing Summary ........................................... 114

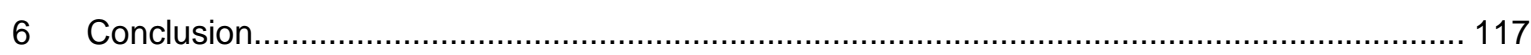

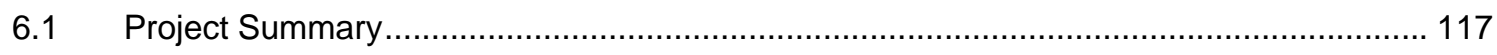

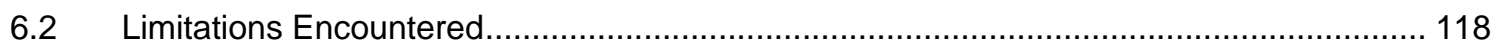

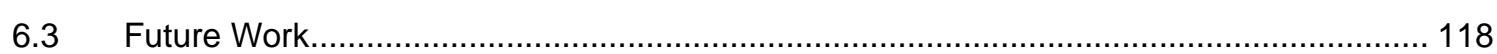

6.4 Bluetooth Wireless Sensor Network Recommendations .......................................... 119

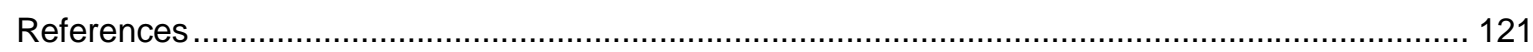

Appendix A: Standard Piconet Bluetooth Network Structure Simulation Code .......................... 127

Appendix B: Bluetooth Piconet Coexistence Simulation Code ............................................ 132

Appendix C: Piconet Sharing Bluetooth Network Structure Simulation Code .......................... 138

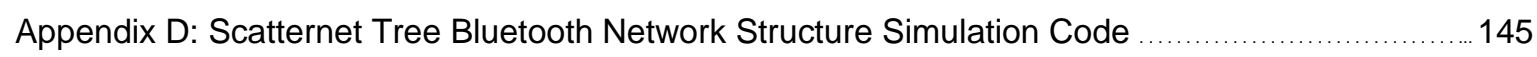

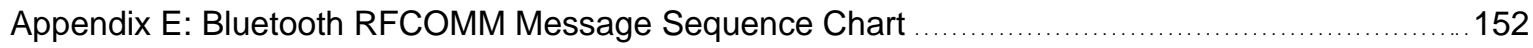

Appendix F: Bluetooth Piconet Sharing Message Sequence Chart .................................... 153

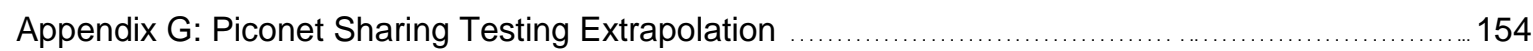

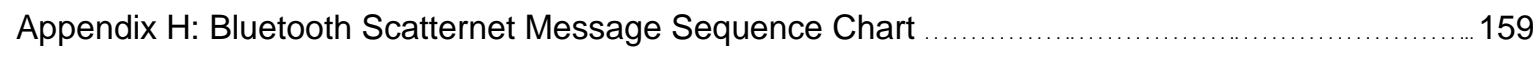

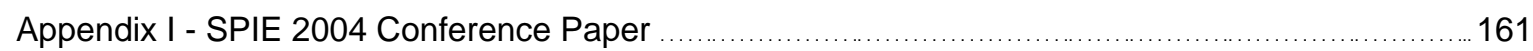


Appendix J: SPIE 2005 Conference Paper

Appendix K: The Japan Society of Mechanical Engineers Conference Paper

190 


\section{List of Acronyms}

This document contains many acronyms. For ease of reading the most commonly used acronyms have been tabulated as Table i, Table ii and Table iii.

\begin{tabular}{|l|l|}
\hline Acronym & Definition \\
\hline PAN & Personal area network \\
\hline LAN & Local Area Network \\
\hline WAN & Wide Area Network \\
\hline WLAN & Wireless Local Area Network \\
\hline WPAN & Wireless Personal Area Network \\
\hline IEEE & Institute of Electrical and Electronics Engineers \\
\hline ECMA & European Computer Manufacturers Association \\
\hline FHSS & Frequency Hopped Spread Spectrum \\
\hline DSSS & Direct Sequence Spread Spectrum \\
\hline MAC & Media Access Controller \\
\hline CSMA/CA & Carrier Sense Multiple Access Collision Avoidance \\
\hline TDMA & Time Division Multiple Access \\
\hline TDD & Time Division Duplex \\
\hline RTS & Request to Send \\
\hline CTS & Clear to Send \\
\hline OSI & Open Systems Interconnect \\
\hline CCK & Complimentary Code Keying \\
\hline BPSK & Bi-polar Phase Shift Keying \\
\hline QPSK & Quadrature Phase Shift Keying \\
\hline QAM & Quadrature Amplitude Modulation \\
\hline OFDM & Orthogonal Frequency Division Multiplexing \\
\hline SIG & Special Interest Group \\
\hline CSR & Cambridge Silicon Radio \\
\hline DEVs & IEEE 802.15.3 Standard Devices \\
\hline PNC & IEEE 802.15.3 Piconet coordinator \\
\hline UWB & Ultra-WideBand (UWB) \\
\hline & \\
\hline
\end{tabular}

Table i: List of Acronyms 


\begin{tabular}{|l|l|}
\hline Acronym & Definition \\
\hline MB-OFDM & Multi Band Orthogonal Frequency Division Multiplexing \\
\hline DS-UWB & Direct Sequence Ultra-WideBand \\
\hline USB & Universal Serial Bus \\
\hline WUSB & Wireless Universal Serial Bus \\
\hline EIRP & Effective Isotropically Radiated Power \\
\hline O-QPSK & Offset Quadrature Phase Shift Keying \\
\hline RFD & IEEE 802.15.4 Reduced Function Devices \\
\hline FFD & IEEE 802.15.4 Full Function Devices \\
\hline NWK & ZigBee Network Layer \\
\hline APL & ZigBee application layer \\
\hline IP & Internet Protocol (IP) \\
\hline WAP & Wireless Access Protocol (WAP) \\
\hline GPS & Global Positioning System (GPS) \\
\hline POLL & Bluetooth poll packet (POLL) \\
\hline EDR & Bluetooth Extended Data Rate (EDR) \\
\hline DMx & Bluetooth Data Medium Packets of length x (DMx) \\
\hline DHx & Bluetooth Data High Packets of length x (DHx) \\
\hline RSSI & receive strength signal indicator (RSSI) \\
\hline ISM Band & Industrial Scientific and Medical Frequency Band (ISM band) \\
\hline BD_ADDR & Unique Bluetooth Address (BD_ADDR) \\
\hline AM_ADDR & Bluetooth Active Member Address (AM_ADDR) \\
\hline PM_ADDR & Bluetooth Parked Member Address \\
\hline AR_ADDR & Bluetooth Access Request Address \\
\hline AFH & Bluetooth Adaptive Frequency Hopping \\
\hline PSK & Phase Shift Keying \\
\hline RF & Radio Frequency \\
\hline HCI & Host Controller Interface \\
\hline LMP & Bluetooth Link Manager Protocol \\
\hline L2CAP & Bluetooth Logical Link Control and Adaption Protocol \\
\hline SDP & Bluetooth Service Discovery Protocol \\
\hline SPP & Bluetooth Serial Port Profile \\
\hline
\end{tabular}

Table ii: List of Acronyms 


\begin{tabular}{|l|l|}
\hline Acronym & Definition \\
\hline APR & Bluetooth Access Point Roaming (APR) \\
\hline M & Bluetooth Piconet Master Node(M) \\
\hline S & Bluetooth Piconet Slave Node (S) \\
\hline M/S & Bluetooth Piconet Master and Slave Node (M/S) \\
\hline ACL & Bluetooth Asynchronous Connection-Less Connection (ACL) \\
\hline SCO & Bluetooth Synchronous Connection-Oriented Connection (SCO) \\
\hline SBC & Single Board Computer (SBC) \\
\hline WinCE & Windows Compact Edition (WinCE) \\
\hline BC02 & Cambridge Silicon Radio BlueCore02 Bluetooth Radio (BC02) \\
\hline
\end{tabular}

Table iii: List of Acronyms 


\section{List of Figures}

Figure 1: A Typical Wireless Sensor Network

Figure 2: Bluetooth network structures; (a) single Piconet structure, (b) master / slave bridging node

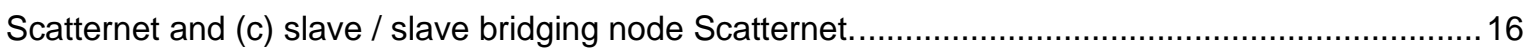

Figure 3: Bluetooth theoretical baseband and quoted RFCOMM throughput per slave $[1,29] \ldots \ldots \ldots . .17$

Figure 4: Bluetooth Stack as outlined by the Bluetooth Specification [1] ........................................ 19

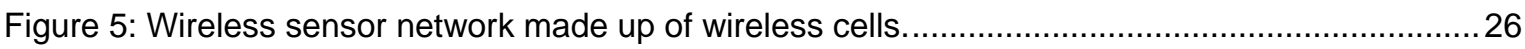

Figure 6: Standard Bluetooth Piconets; (a) Point to Point Connection and (b) Point to Multi-Point

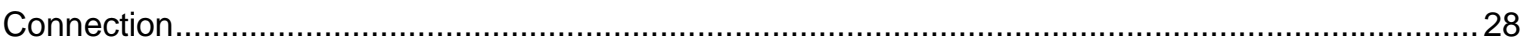

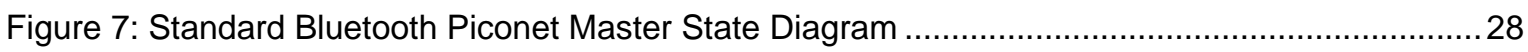

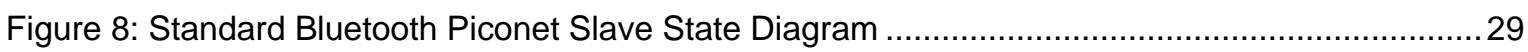

Figure 9: Piconet Sharing Bluetooth Network Structure; (a) Group 1 is Active and Group 2 is Parked

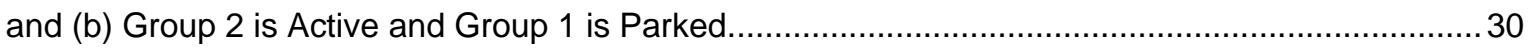

Figure 10: Bluetooth Piconet Sharing Master State Diagram ......................................................... 31

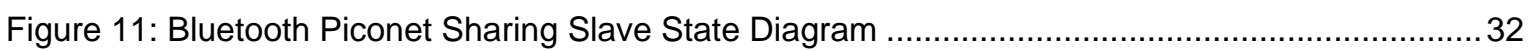

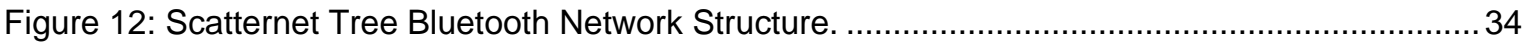

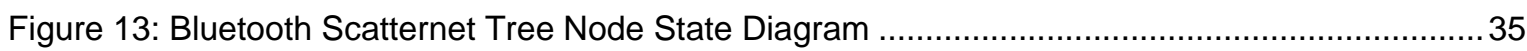

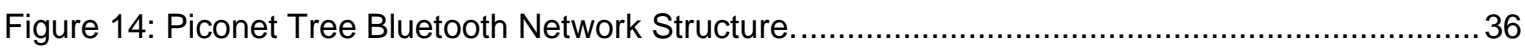

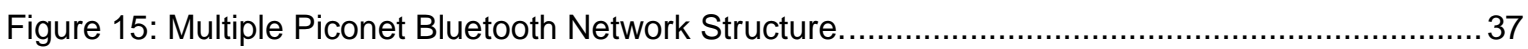

Figure 16: Example of Channel Access on a Bluetooth Piconet with Two Active Slaves..................... 42

Figure 17: Standard Bluetooth Piconet Network Structure Simulation Main Flow Diagram ................44

Figure 18: Standard Bluetooth Piconet Network Structure Simulation Flow Diagram ...........................45

Figure 19: Sample Output for Standard Bluetooth Piconet Simulation ............................................. 46

Figure 20: Example Standard Piconet Simulation Throughput per Slave Output ...............................47

Figure 21: Standard Bluetooth Piconet Simulation Network Throughput Results............................... 48

Figure 22: Bluetooth Piconet Coexistence Simulation Main Flow Diagram ....................................... 51

Figure 23: Bluetooth Piconet Coexistence Simulation Flow Diagram ............................................. 52

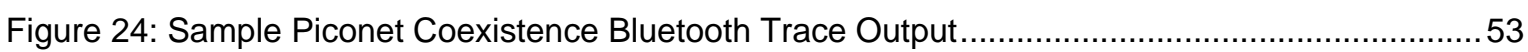

Figure 25: Sample Piconet Coexistence Simulation Throughput per Slave Output..............................54

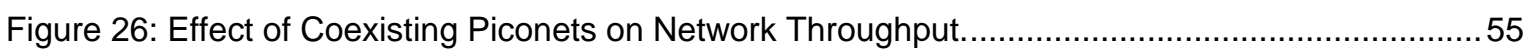

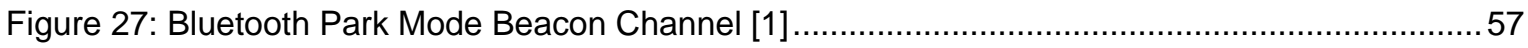

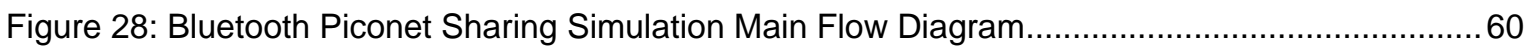

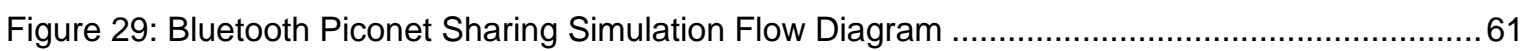

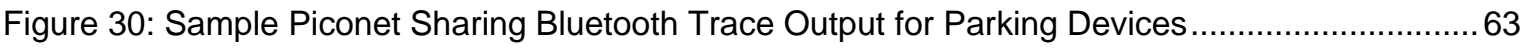

Figure 31: Sample Piconet Sharing Bluetooth Trace Output for Park Beacon ..................................... 63 
Figure 32: Sample Piconet Sharing Bluetooth Trace Output for Unparking Devices with Parking Beacon 64

Figure 33: Sample Piconet Sharing Simulation Throughput per Slave Output.

Figure 34: Average Throughput Results for Bluetooth Piconet Sharing Simulation for Differing

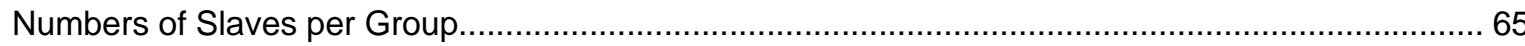

Figure 35: Average Throughput Results for Bluetooth Piconet Sharing Simulation ............................. 66

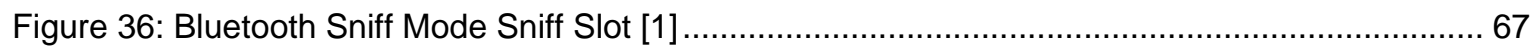

Figure 37: Bluetooth Scatternet Tree Structure Simulation Main Flow Diagram .................................. 69

Figure 38: Bluetooth Scatternet Tree Structure Simulation Flow Diagram...................................... 70

Figure 39: Example Scatternet Tree Bluetooth Trace Output.................................................... 72

Figure 40: Example Bluetooth Scatternet Tree Structure with Optimum Sniff Timeout; (a) Throughput per Node Output and (b) Master / Slave Node Data Buffer .............................................................. 73

Figure 41: Example Bluetooth Scatternet Tree Structure with Too Large Sniff Timeout; (a)

Throughput per Node Output and (b) Master / Slave Node Data Buffer ........................................ 73

Figure 42: Example Bluetooth Scatternet Tree Structure with Too Small Sniff Timeout; (a)

Throughput per Node Output and (b) Master / Slave Node Data Buffer ........................................ 74

Figure 43: Average Throughput Results for Bluetooth Scatternet Tree Structure Simulation.............. 75

Figure 44: Throughput Results for Bluetooth Piconet Tree Network Structure Simulation ................. 77

Figure 45: Throughput Results for Multiple Piconet Network Structure Simulation............................. 78

Figure 46: Bluetooth Hardware Used in Wireless Sensor Testing; (a) Bluetooth Root Node [64] and

(b) Wireless Sensor Node (Radio Only) [65]

Figure 47: Bluetooth Root Node and Wireless Sensor Node Stack Architecture (a) Single Board

Computer and Bluetooth Module ( $\mathrm{HCl}$ Firmware) and (b) Sensor Platform and Bluetooth Module (RFCOMM Firmware)

Figure 48: Bluetooth Device Connection and Reconnection Testing ............................................ 87

Figure 49: Bluetooth Unparking Times for Varying Park Beacon Lengths .......................................... 88

Figure 50: Throughput Drop Off with Increases Communication Range ......................................... 90

Figure 51: Measured Throughput per Slave for Root Node Created Standard Piconet...................... 91

Figure 52: Measured Throughput per Slave for Bluetooth Module Created Standard Piconet ........... 92

Figure 53: Piconet Sharing Node Extrapolation (a) Extrapolated Piconet Sharing Structure and (b)

Actual Tested Piconet Sharing Structure 94

Figure 54: Extrapolated Bluetooth Piconet Sharing Network Structure Minimum Throughput............ 98

Figure 55: Effect of Average Piconet Sharing Throughput of Piconet Sharing Group Active Time..... 99

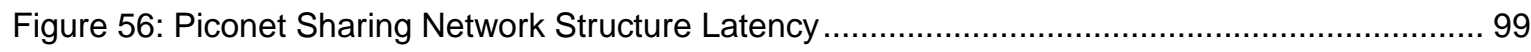

Figure 57: Extrapolated Bluetooth Scatternet Tree Network Structure Minimum Throughput ........... 104 
Figure 58: Bluetooth Piconet Tree Stack Architecture (a) Piconet Tree Bridging Node Stack Architecture and (b) Piconet Tree Sensing Node Stack Architecture 105

Figure 59: Extrapolated Bluetooth Piconet Tree Network Structure Minimum Throughput ............... 109

Figure 60: Bluetooth Multiple Piconet Network Structure Stack Architecture ................................. 110

Figure 61: Extrapolated Bluetooth Multiple Piconet Network Structure Minimum Throughput ...........113

Figure 62: Proposed Advanced Host Control Interface Bluetooth Stack Layer .................................. 114 


\section{List of Tables}

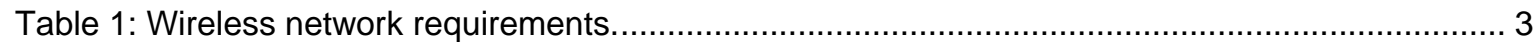

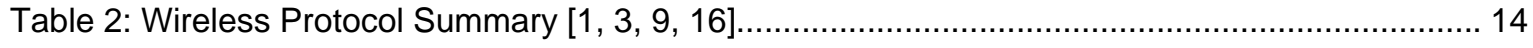

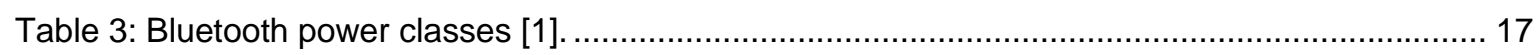

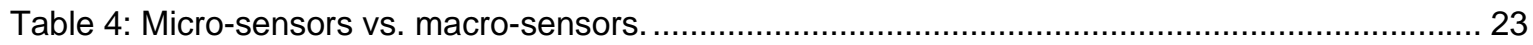

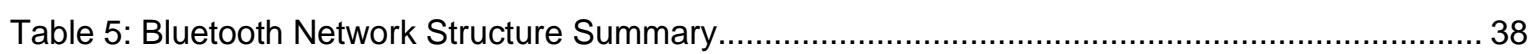

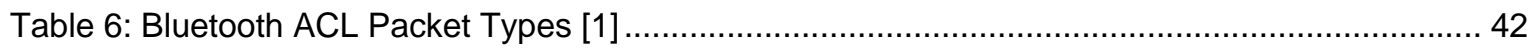

Table 7: Standard Bluetooth Piconet Network Structure Simulation Parameters................................ 45

Table 8: Comparison Between Simulation Results and Bluetooth Specification ................................. 47

Table 9: Bluetooth Piconet Coexistence Simulation Parameters ....................................................... 52

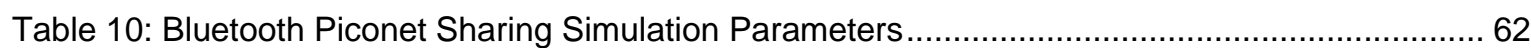

Table 11: Bluetooth Scatternet Tree Simulation Parameters ……............................................. 71

Table 12: Bluetooth Scatternet Tree Network Structure Possible Node Combinations ........................ 75

Table 13: Bluetooth Piconet Tree Network Structure Possible Node Combinations ............................. 76

Table 14: Bluetooth Multiple Piconet Network Structure Possible Node Combinations ....................... 78

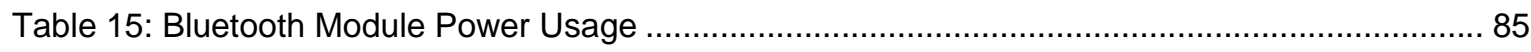

Table 16: Bluetooth Module Power Usage in Bluetooth Low Power States ......................................... 86

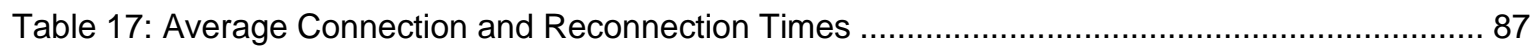

Table 18: Average Unparking Times for Varying Park Beacon Lengths ............................................ 89

Table 19: Extrapolated Piconet Sharing Hardware Testing Results............................................... 95

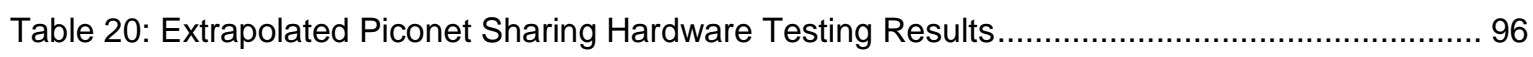

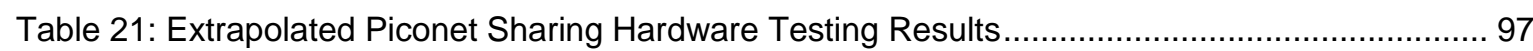

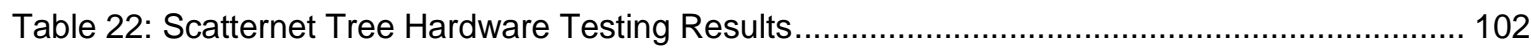

Table 23: Extrapolated Scatternet Tree Hardware Testing Results .............................................. 103

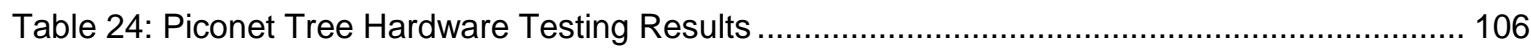

Table 25: Extrapolated Piconet Tree Hardware Testing Results.................................................... 107

Table 26: Extrapolated Piconet Tree Hardware Testing Results................................................ 108

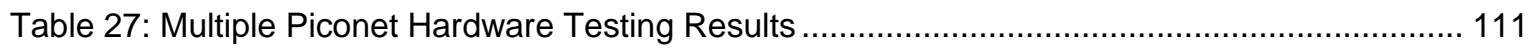

Table 28: Extrapolated Multiple Piconet Hardware Testing Results.............................................. 112 


\section{Introduction}

Wireless networks allow communication between nodes which are unable to be networked via traditional wired means. This may be due to the large cost in setting up a wired infrastructure or the nodes may be mobile making wires impossible. A wireless network of sensor nodes can be deployed quickly and easily, allowing near real time feedback of collected data. A wireless network appropriate for sensing applications must be able to handle the required data throughput, required range, support the required number of nodes and use a minimum amount of power to conserve battery life. The wireless sensor network developed in this thesis is for sport monitoring applications. Creating a wireless network of sensor platforms allows athletes and coaches near real time feedback on any number of sporting parameters. This means that coaches have a chance to correct an athlete's technique on the fly and to also have a record of an athletes training session without having to manually record the athletes movements. A wireless sensor network allows sensor platforms to record performance data with synchronisation between data sets for many athletes. When sensor nodes are deployed on mobile objects, the wireless network must handle node dropouts and reconfigure itself for the constantly changing network.

Wireless sensor networks are different from traditional wireless ad-hoc networks. Ad-hoc networks are designed to be created "on the fly" with any number of unknown nodes and are typically used for data transfer between any of the nodes. Wireless sensor networks are typically deployed with a known number of nodes, with the nodes being known prior to network initialization. The nodes usually don't need to communicate with each other, the aim of sensor networks is to collate the data in a central location for analysis. Figure 1 shows a typical wireless sensor network with mobile sensor nodes, fixed wireless access points, a high speed data backbone and a central data server. The Bluetooth Wireless sensor network was designed with these things in mind, to ensure optimum network performance.

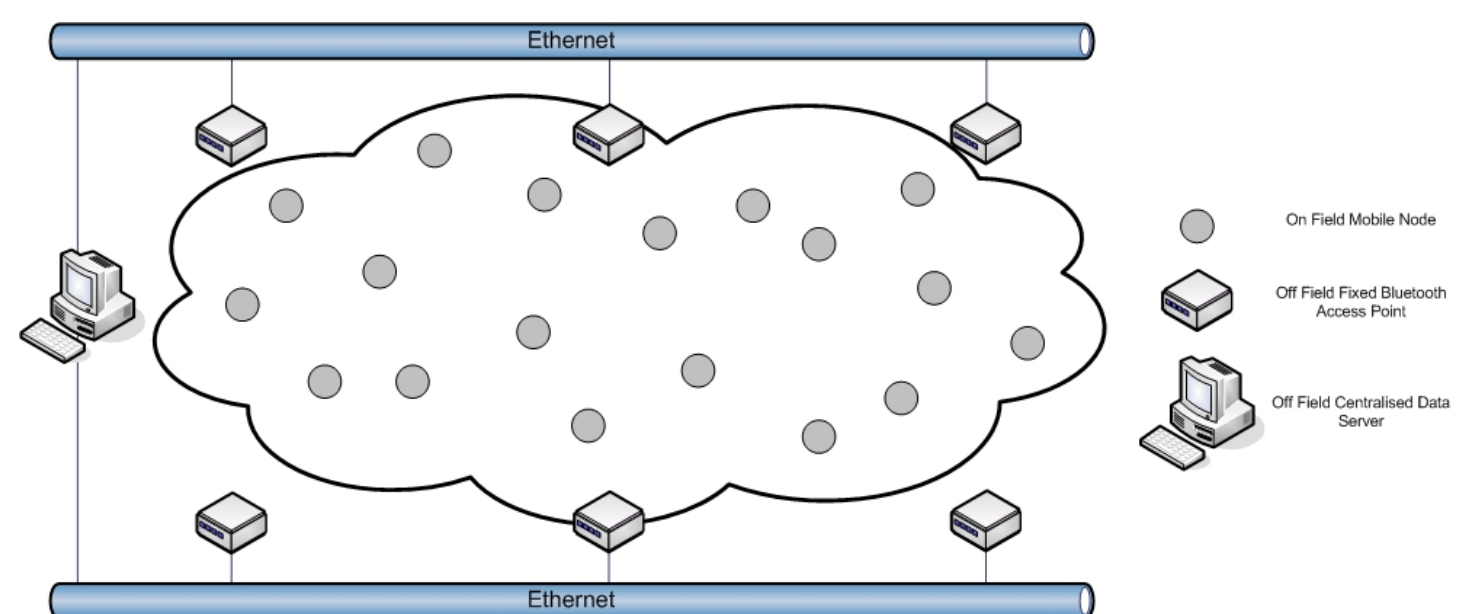

Figure 1: A Typical Wireless Sensor Network

- 1 - 
The Bluetooth Specification was created to provide a cable replacement solution for fixed and mobile devices [1]. It provides a number of upper layer profiles that provide automatic service discovery of a device's capabilities and ensures interoperability between devices designed by different manufacturers. A major draw back of the Bluetooth technology for wireless sensor networks is the limitation of eight active devices on a Bluetooth network. The focus of this research was to develop a series of Bluetooth based network structures implemented with a number of fixed access points. This solution creates a viable Bluetooth based wireless sensor network for sports monitoring applications. Building the wireless sensor network using the Bluetooth Specification gives the advantages of readily available and inexpensive hardware, an already established robust communications protocol and compatibility with existing Bluetooth devices (personal digital assistants, mobile phones and Bluetooth access points). The major disadvantage of using the Bluetooth Specification is being constrained to a maximum of seven active slaves (eight devices including the master) in a Bluetooth network (Piconet).

\subsection{Research Objectives}

The main objective of this research was the delivery of a wireless network of multiple sensor nodes for monitoring large numbers of mobile agents built upon the Bluetooth Specification. The Bluetooth Specification has been selected for the wireless sensor network to remain compatible with existing hardware and previously implemented wireless monitoring solutions. Increasing the node limits of the existing Bluetooth based wireless monitoring equipment will expand the usefulness of these devices, allowing them to be used in a larger number of monitoring scenarios.

Standard Bluetooth networks (Piconets) only supports up to eight active devices. This research provided several Bluetooth network structures to support more than 40nodes. These structures allowed the Bluetooth Specification to be used for the desired team sports monitoring application.

A literature review of current wireless network protocols was completed, along with current research into wireless networks and wireless sensor networks.

The produced wireless sensor network was designed to be immediately implemented for use in athlete and sports monitoring. For this reason considerations like currently available hardware capabilities and vendor stack implementations were investigated. This ensured that the proposed Bluetooth network structures were able to be implemented with current Bluetooth hardware and software.

The delivered wireless network provided an adequate data rate for the application, was able to support many wireless nodes and was a low power solution. Novel Bluetooth network structures were developed to satisfy all of these requirements. These structures were simulated, implemented and tested with currently available versions of the Bluetooth Specification. 


\section{$1.2 \quad$ Prior Work}

The wireless sensor network has been developed for use with an existing integrated microprocessor sensor platform used for sports monitoring applications [2]. Wireless networking of this platform allowed near real time analysis of the collected data. The platform was made up of a microprocessor that sampled on-board accelerometers and logs the acquired data to an attached flash memory card. The three channels of acceleration data are typically logged with a 10 bit resolution 150 times a second (a total of $4500 \mathrm{bps})$. The developed wireless sensor network was required to support this data rate. Networking these sports monitoring platforms also allowed multiple athlete monitoring, as the network provides a method for data synchronisation across all nodes.

\subsection{Wireless network requirements}

Typically ad-hoc wireless networks are designed for data communications between any of the nodes. Wireless networks of sensor nodes require the data to be collated at a single fixed receiving node for analysis and storage. These two different scenarios have different solutions. Typically the configuration in ad-hoc networks is handled by the wireless nodes themselves. This process can be computationally intensive and means that the nodes themselves can never enter an energy saving low power state. The proposed network topology used the fixed receiving nodes to configure the network, as they typically have greater processing power than the wireless nodes. The fixed receivers also had knowledge of all the wireless nodes and there was no need to search for additional nodes before making changes to the topologies layout. A summary of the wireless network requirements is given in Table 1.

\begin{tabular}{|l|l|}
\hline Network Property & Metwork Requirement \\
\hline Number of nodes & Medium battery life (at least 4 hrs of operation) \\
\hline Power Consumption & Medium data rate (sensor nodes require 4500 bps) \\
\hline Data Rate & Large area to be monitored (at least $5000 \mathrm{~m} 2)$ \\
\hline Range & Near real time monitoring required \\
\hline Data Access &
\end{tabular}

Table 1: Wireless network requirements.

\section{$1.4 \quad$ Thesis Outline}

This thesis consists of five sections that entail the body of work completed on the Bluetooth based wireless sensor networks. Chapter 2 presents an introduction to available wireless standards and examines their usefulness in wireless sensor networks. The certified IEEE wireless local area network (WLAN) and wireless personal area network (WPAN) standards were reviewed along with several IEEE standards that are still in development. A literature review of current research assessed published - 3 - 
research on wireless sensor networks, research currently undertaken to improve the Bluetooth specification and specific implementations of the Bluetooth specification. Version 1.1 of the Bluetooth specification is summarised and the key features that affect Bluetooth's implementation in wireless sensor networks are highlighted. Differences between Bluetooth version 1.1, 1.2 and 2.0 are also explained.

Chapter 3 outlines the developed Bluetooth systems and structures for wireless sensor networks. Developed structures include Piconet Sharing, Scatternet Tree Structure, Piconet Tree Structure and Multiple Radio Root Nodes. Implementation of these Bluetooth structures into a complete wireless sensing system has been described, including the use of multiple root nodes and Bluetooth hand off schemes.

Bluetooth simulations were created to demonstrate the performance of the proposed Bluetooth structures. A complete description of the MATLAB based Bluetooth simulations can be found in Chapter 4 along with a list of assumptions made, validation methods and simulation results. Conclusions drawn from the simulation results demonstrate how to optimise the Bluetooth sensor network under different conditions.

The developed structures were implemented with existing Bluetooth hardware and the results are included in Chapter 5. An initial overview of the hardware used and initial hardware testing demonstrates additional limitations that are not inherit in the Bluetooth specification. A description of each of these structures follows, including testing conditions and testing assumptions made. Testing results are presented for each structure and conclusions drawn about their usefulness in wireless sensor networks.

Chapter 6 concludes the thesis with an overview of results and conclusions made throughout the document. Possible future work has been described, including switching the operating system on the root nodes and methods for customising the Bluetooth stack for wireless sensing applications. Finally recommendations are made about which structures are viable with currently available Bluetooth hardware for use in wireless sensor networks.

\subsection{Research Outcomes}

This body of work produced several original research outcomes that were published in several conference papers.

\subsubsection{Original Contributions}

The major original contributions of this body of work are:

- A cutdown MATLAB simulation of communications on a Bluetooth Piconet

- Thorough testing of selected Bluetooth hardware, exposing undocumented limitations in Bluetooth hardware implementation 
- Designed, simulated and experimentally tested four novel Bluetooth Network Structures

- Proposed Advanced Host Control Interface (AHCl) Bluetooth stack layer that allows multiple Bluetooth radios.

\subsubsection{List of Publications}

The following publications have been written from this body of work:

- M. J. Fraser, D. A. James and D. V. Thiel, Wireless Network Topology for Monitoring Mobile Agents, Proceedings of SPIE vol 5651 Biomedical Applications of Micro- and Nanoengineering II, Sydney, NSW, 2004

- M. J. Fraser, D. A. James and D. V. Thiel, Innovative techniques for extending the range and node limits in Bluetooth-based wireless sensor networks, Proceedings of SPIE vol 6035 Microelectronics: Design, Technology and Packaging II, Brisbane, QLD, 2005

- D. A. James and M.J. Fraser, The application of wireless technology and sensors for sporting applications, The Japan Society of Mechanical Engineers (Bioengineering Division), Sports Engineering Symposium, Kanazawa, 2006 


\section{Wireless Technologies and Literature Review}

The following review of literature provides a background for this body of work in wireless networks and sensor networks. Although the Bluetooth specification was mandated as the wireless standard for the sensor network, for completeness an overview of the currently available wireless protocols was conducted. The Bluetooth Specification has been outlined along with an overview of research that has previously been undertaken involving the Bluetooth specification. A review of current research into wireless biomonitoring and sports monitoring was undertaken and has been presented.

\subsection{Wireless Protocol Review}

This body of work was focused on creating a feasible Bluetooth based wireless sensor network, however there are a number of alternate wireless specifications and protocols commercially available. The Institute of Electrical and Electronics Engineers (IEEE) Standards Association has three different wireless network standards; IEEE 802.11 Wireless, IEEE 802.15 Wireless Personal Area Networks (WPANs) and IEEE 802.16 Broadband Wireless Metropolitan Area Networks. The following wireless protocols are currently being used for wireless sensor networks. Highlights of some emerging technologies that are set to be used in wireless sensor networks in the future have also been included.

\subsubsection{Wireless Local Area Networks (IEEE 802.11)}

In 1997 the IEEE ratified the 802.11 wireless standard for wireless local area networks (WLAN). The original IEEE 802.11 standard had two alternate physical layers based on frequency hopped spread spectrum (FHSS) and direct sequence spread spectrum (DSSS) both operating in the $2.4 \mathrm{GHz}$ ISM band. The major draw back to the original wireless standard was the lack of interoperability between the two different physical layers and was limited to a maximum data rate of 2 Mbps. These problems were rectified with the release of the IEEE 802.11b amendment in 1999. IEEE 802.11a was released shortly afterward operating in the $5.8 \mathrm{GHz}$ band allowing greater data throughput. IEEE $802.11 \mathrm{~g}$ was released in 2003 and shares the same frequency band as IEEE 802.11b. IEEE 802.11g uses a more advanced modulation scheme allowing a similar throughput to IEEE 802.11a while remaining backwards compatible with IEEE 802.11b [3].

The media access method (MAC) used in all IEEE 802.11 network types is described as carrier sense multiple access collision avoidance (CSMA/CA). In a CSMA/CA wireless network, a node senses if other devices are transmitting before transmitting data. If the media is currently in use by another device, the node waits for the media to become available before transmitting. Request to send (RTS) and clear to send (CTS) CSMA/CA handshaking packets help eliminate the potential "hidden node" problem. Even If a $-6-$ 
node can't hear another node transmitting it will still back off transmitting when it receives another nodes CTS packet.

IEEE 802.11 networks use the IEEE standard 48 bit addressing scheme that compiles with the guidelines set out by the IEEE Computer Society [4]. The IEEE 802.11 standards define layers 1 and 2 (Physical and Medium Access Control layers) of the Open Systems Interconnect (OSI) model and the upper layers are identical to IEEE 802.3 and 802.5 Ethernet standards. This means that the IEEE 802.11 standard is inherently supported by most personal computing operating systems.

\subsubsection{IEEE 802.11b}

The IEEE 802.11b amendment was implemented to overcome the problems with the original IEEE 802.11 and gained popularity as wireless computing started to become popular. IEEE 802.11b operates in the 2.4 GHz ISM band and has a theoretical baseband throughput of $11 \mathrm{Mbps}$ per channel, with a typical application level throughput of about 4 Mbps [3]. Typically IEEE 802.11b networks have a range of 100 meters with omnidirectional antennas. The physical layer of IEEE 802.11b uses complimentary code keying (CCK) modulated on to one of 14 separate channels. These channels overlap and have an approximate bandwidth of $22 \mathrm{MHz}$. To achieve maximum throughput with an IEEE 802.11b network channels 1,6 and 11 can be used by three separate IEEE 802.11b access points. These channels typically don't overlap (depending on the transmit power on each channel) and a maximum total of 33 Mbps baseband throughput can be achieved for the total wireless network [3]. IEEE 802.11b wireless network interface cards are available from almost any computer store and hardware development modules and single chip solutions are also readily available for use in embedded systems.

\subsubsection{IEEE 802.11a}

The IEEE 802.11a amendment was ratified shortly after IEEE 802.11b. IEEE 802.11a physically operates in the 5.8Ghz ISM band [3]. This has the advantage of operating in a relatively empty unused band as most other wireless technologies (IEEE 802.11b, IEEE 802.11g, Bluetooth, IEEE 802.15.4, etc) utilise the $2.4 \mathrm{GHz}$ ISM band. A disadvantage at operating at such a high frequency is that the signal along the path is attenuated by obstacles such as walls and furniture. This means additional access points may be needed to cover the same space as a network operating in the $2.4 \mathrm{GHz}$ band. The baseband data is modulated using bi-polar phase shift keying (BPSK), quadrature phase shift keying (QPSK) or quadrature amplitude modulation (QAM) using orthogonal frequency division multiplexing (OFDM) on 52 sub carriers per channel. The maximum possible baseband throughput per channel is 54 Mbps, with an average of approximately $20 \mathrm{Mbps}$ application level throughput usually achieved. There are a total of twelve non-overlapping channels each with a bandwidth of $20 \mathrm{MHz}$. Eight of these channels are dedicated to indoor communications and the remaining four are for point to point communications. 
These channels can be utilised by eight multiple access points to obtain an overall maximum baseband throughput of $432 \mathrm{Mbps}[3,5]$. IEEE 802.11a was less popular due to the inherit disadvantages of using a higher frequency, poor initial implementations and the fact that IEEE $802.11 \mathrm{~b}$ already had such a firm hold on the market.

\subsubsection{IEEE 802.11g}

In 2003 the third IEEE 802.11 radio standard was ratified, IEEE 802.11g. IEEE 802.11g operates in the same $2.4 \mathrm{GHz}$ ISM frequency band as IEEE $802.11 \mathrm{~b}$ and products are backwards compatible with IEEE 802.11b devices. IEEE 802.11g utilises OFDM in a similar manner as IEEE 802.11a and has a similar theoretical baseband throughput of $54 \mathrm{Mbps}$ per channel. As IEEE 802.11g uses the same channels as IEEE 802.11b it has three non-overlapping channels. If these channels are utilized by three different access points an implemented IEEE 802.11g network can have a maximum total throughput of $162 \mathrm{Mbps}$ [5]. In practice IEEE $802.11 \mathrm{~g}$ has a greatly reduced throughput due to coexistence issues in the already heavily populated $2.4 \mathrm{GHz}$ ISM band with Bluetooth, IEEE 802.11b, cordless telephones and microwave ovens. Some IEEE $802.11 \mathrm{~g}$ products come with proprietary modifications to the standard that improve throughput. An example of this is the Super $G$ technology implemented by the integrated circuit manufacturer Atheros [6]. Theoretical baseband data rates of up to $108 \mathrm{Mbps}$ can be achieved by using a combination of reducing the packet overhead, further compressing the data, using fast frames (bundling two data frames into one) and by using channel bonding to utilise two IEEE 802.11g channels at once. Channel bonding also increases the maximum range of transmission [6]. Similar technologies are implemented by other chip manufacturers.

\subsubsection{Wireless Personal Area Networks (IEEE 802.15)}

The IEEE 802.15 working group was established for the development and ratification of standards in wireless personal area networking (WPAN) [7]. The working group was originally broken up into four task groups Bluetooth (IEEE 802.15.1), coexistence issues (IEEE 802.15.2), WPAN High Rate (IEEE 802.15.3) and WPAN Low Rate (IEEE 802.15.4). Subsequently after the ratification of the IEEE 802.15.3 and 802.15.4 standards, several additional subtasks were formed. IEEE 802.15.3a WPAN Alternate High Rate task was formed to ratify an alternate physical layer to the IEEE 802.15.3 standard based on ultrawide band technology. IEEE 802.15.4a WPAN Alternate Low Rate task was formed to ratify an alternate physical layer. Both the IEEE 802.15.3b and 802.15.4b subtasks are aimed at producing ongoing improvements to the standard [7].

WPANs are made up of small networks of devices referred to as Piconets. A Piconet is an adhoc network of personal area devices that is confined to a small area (typically 10 meters in all directions) [8]. This is 
in contrast to local area networks (LANs) and wide area networks (WANs) that cover a much larger area (hundreds of meters) and are usually planned (WPANs are typically adhoc networks).

\subsubsection{Bluetooth (IEEE 802.15.1)}

In 1998 Ericsson, Nokia, IBM, Toshiba and Intel formed the Bluetooth Special Interest Group (SIG) dedicated to providing interoperability between Bluetooth devices [9]. Bluetooth is being hailed as the cable replacement solution for short range local networks. Bluetooth operates in the $2.4 \mathrm{GHz}$ ISM band and provides a baseband throughput of $723.2 \mathrm{kbps}$. Bluetooth uses frequency hopped spread spectrum (FHSS) as its coexistence technology, dividing the ISM band into 79 channels which it hops between at a rate of 1600 hops per second [1].

There are three radio power classes of Bluetooth devices; class 1 long range devices have a maximum radiated power of $100 \mathrm{~mW}$ (approximately 100 meters range), class 2 normal range devices have a maximum radiated power of $2.5 \mathrm{~mW}$ (approximately 10 meters range) and class 3 short range devices have a maximum radiated power of $1 \mathrm{~mW}$ (approximately 1 meter range).

Bluetooth Piconets consist of one device acting as a master and up to seven devices acting as active slaves. The master controls communication on the network using a time division polling scheme. The slaves on the network are unaware of each other and can only transmit data to the master [1]. Bluetooth has a number of defined profiles that ensure interoperability between devices by different manufacturers. Bluetooth modules are readily available for easy integration into embedded systems and there are a number of single chip Bluetooth solutions available. The majority of the Bluetooth solutions contain one of the BlueCore range of single chip Bluetooth solutions made by Cambridge Silicon Radio (CSR) [10].

\subsubsection{High Rate Wireless Personal Area Networks (IEEE 802.15.3)}

The IEEE 802.15.3 task group was established to develop a WPAN standard that provides a solution for applications that require a higher data rate solution than Bluetooth (IEEE 802.15.1) can provide. Adhoc node discovery and a Piconet structure are still a requirement of this standard and this differentiates it from WLAN specifications. The physical layer operates in the $2.4 \mathrm{GHz}$ ISM band and supports two different channel plans. The first uses four channels for high density applications and the other uses three bands for better coexistence with IEEE 802.11b. The maximum baseband data rate for each channel is $55 \mathrm{Mbps}$, using trellis code modulation. IEEE 802.15.3 coexists in an already heavily utilised frequency band and has been designed with multiple techniques to aid in coexistence including dynamic channel selection, link quality indicators and a channel plan that minimises overlap [8].

The IEEE 802.15.3 Piconet is made up of two kinds of devices standard devices (DEVs) and a device acting as the Piconet coordinator (PNC). The PNC provides a beacon to the Piconet, manages power

$$
\text { - } 9 \text { - }
$$


save modes and access to the Piconet. All communications on an IEEE 802.15.3 Piconet using a peer to peer communications style, but are controlled via the PNC's superframe. The superframe divides communications on the Piconet into a contention access period where communications are CSMA/CA and a channel time allocation period where communication occurs in a time division multiple access (TDMA) scheme. Devices in the Piconet can also request to form their own dependant Piconet, which relies on the parent PNC to allocate channel time. A Piconet which doesn't have any dependant Piconets is referred to as a dependant Piconet [8].

\subsubsection{Alternate High Rate Wireless Personal Area Networks (IEEE 802.15.3a)}

The IEEE 802.15.3a working group was formed to provide an alternate physical layer for the IEEE 802.15.3 specification that supports data rates appropriate for imaging and multimedia. There were two proposed physical layers both based on ultra-wideband (UWB) technologies; WiMedia's multi band orthogonal frequency division multiplexing (MB-OFDM) and the UWB forum's direct sequence ultrawideband (DS-UWB). The IEEE 802.15.3a task force has been trying to decide on both of these standards for over two years and in early 2006 the task force was disbanded due to no conclusion being able to be reached. Both UWB technologies support a maximum baseband throughput of up to 480 Mbps, making the technologies suitable for media streaming applications and wireless cable replacement for Universal Serial Bus (USB) 2.0 and Firewire devices [11, 12].

The WiMedia alliance is backed by some of the biggest technology industry companies such as Microsoft, Sony, Phillips, and Nokia to name just a few [11]. Prominent industry special interest groups (SIG) such as the Bluetooth SIG and the Wireless USB (WUSB) Promoter Group have also joined the WiMedia alliance, with the WUSB specification being built upon the WiMedia MAC and Physical layer and the Bluetooth SIG is expected to do the same.

Two of the founding members of the UWB Forum were Freescale and Motorola, but it seems that after the backing of the WiMedia specification by the Bluetooth SIG and the WUSB Promoter Group they have left the UWB Forum to focus solely on cable free USB applications [13]. Only time will tell how this development will effect the UWB Forum's ability to compete with the heavily backed WiMedia Alliance.

While the IEEE 802.15.3a working group was unable to ratify an alternate high rate physical layer based on one of these two technologies, the European Computer Manufacturers Association (ECMA) in December 2005 ratified ECMA-368 High Rate Ultra Wideband PHY and MAC Standard. This standard is based heavily on the WiMedia Alliance's MB-OFDM specification. The ECMA-368 standard defines the UWB spectrum over the frequency range $3.1 \mathrm{GHz}$ to $10.6 \mathrm{GHz}$ and the splits the spectrum into 5 band groups. Each band group is made up of three bands (except the last band group which is made up of 2 bands), with each band having a bandwidth of $528 \mathrm{MHz}$. Each band uses a total of 110 sub-carriers to 
transmit the information (100 data carriers and 10 guard carriers). The standard also outlines optional requirements for providing ranging and location awareness functionality. This is achieved by measuring the propagation delay between two devices and multiplying by the speed of light. Where ranging is supported a minimum accuracy of $60 \mathrm{~cm}$ or better must be achievable. The ECMA-368 MAC layer provides functionality similar to the IEEE 802.15.3 MAC with both TDMA and CSMA functionality being supplied by the networks superframe [14].

There are a number of licensing concerns with the implementation of UWB that will inevitably delay the specification from appearing in consumer electronics in the short term. As both UWB technologies use the already utilised bandwidth of $3.1 \mathrm{GHz}$ to $10.6 \mathrm{GHz}$, many people are concerned about the interference UWB based devices will have on other devices operating in this band. In 2002 the FCC approved a spectral mask for the operation of UWB devices defining the maximum effective isotropically radiated power (EIRP) allowed for UWB as $-41.3 \mathrm{dBm}$ per $\mathrm{MHz}$ [15]. While this enables UWB devices to operate in the United States of America legally, a number of other countries are insisting that UWB chip manufacturers provide further spectral masks in their radios that will null out certain frequency bands deemed to be the most likely to suffer from UWB interference.

\subsubsection{Low Rate Wireless Personal Area Networks (IEEE 802.15.4)}

The IEEE 802.15.4 standard for low data rate communications was created for low data rate, low cost and long battery life. IEEE 802.15.4 operates with 27 individual channels in three unlicensed frequency bands, one channel at $868 \mathrm{MHz}, 10$ channels in the $915 \mathrm{MHz}$ band and 16 channels in the $2.4 \mathrm{GHz}$ ISM band. The $868 \mathrm{MHz}$ and $915 \mathrm{MHz}$ bands use DSSS and BPSK modulation, with a maximum baseband data rate of $20 \mathrm{kbps}$ per channel ( $868 \mathrm{MHz}$ band) and $40 \mathrm{kbps}$ per channel (915 MHz band). Communication in the $2.4 \mathrm{GHz}$ band uses a 16-ary quasi-orthogonal modulation technique and the chip sequence is modulated onto the carrier using offset quadrature phase shift keying (O-QPSK). Each channel in the $2.4 \mathrm{GHz}$ band has a maximum baseband data rate of $250 \mathrm{kbps}$ [16]. Channels in IEEE 802.15.4 MHz do not overlap as they do in the IEEE 802.11 b and 802.11g standards.

The MAC method used is CSMA/CA which is the same as used in IEEE 802.11 networks; however there is the provision to allocate guaranteed time slots to nodes with time critical data. This is achieved through an optional super frame similar to that implemented in IEEE 802.15.3. The addressing scheme used is either a 16 bit short address or the standard IEEE 64 bit addressing scheme [17]. Devices in an IEEE 802.15.4 network are described as reduced function devices (RFD) or full function devices (FFD). The network is created by a FFD acting as the PAN coordinator node. Network topologies are either a star configuration with all data being routed through the PAN coordinator (similar to Bluetooth networks) or communications occur on a peer to peer basis. FFDs can communicate with the PAN coordinator, other 
FFD coordinator nodes or RFDs. RFDs can only communicate with FFDs. This reduced functionality of RFDs allows for reduced power operation and allows for implementation on a device with limited resources [16]. It is through these flexible network topologies that mesh networking is possible with IEEE 802.15.4 devices. The ZigBee alliance has defined the upper layer network for the discovery, creation and manipulation of IEEE 802.15.4 based mesh networks.

\subsubsection{ZigBee Wireless Sensor Networks (IEEE 802.15.4 based)}

The ZigBee Alliance released version 1.0 of the ZigBee specification in December 2004. The specification outlines the upper network layers of the OSI model for a self healing wireless mesh sensor network built upon the IEEE 802.15.4 MAC and physical layers. The ZigBee Network (NWK) Layer sits atop the IEEE 802.15.4 MAC layer and provides routing of packets through the mesh network using either tree routing or table routing modes. The NWK layer provides the 16 bit unique network device address for use in data routing. This limits the total number of possible nodes throughout a ZigBee network to 65536. The application (APL) layer sits above the NWK layer and provides functions such as device and service discovery, binding management, node management and supports the various sensors endpoints (such as sensors and actuators). The APL layer does this through the application support sub-layer (APS), application framework (AF) and ZigBee device object (ZDO) sublayers [18].

There are three types of ZigBee devices; the ZigBee coordinator (ZC) which controls the Piconet, ZigBee routers $(Z R)$ which are able to route data through the network and ZigBee end devices (ZED) which are limited in functionality. The ZC and ZRs are IEEE 802.15.4 full function devices (FFD) and the ZED is a reduced function device (RFD). In typical ZigBee implementations if possible the mobile devices are implemented as ZEDs to conserve the battery life of the device. A battery life of years is typical with a single alkaline battery [19].

Recently single chip solutions have become available with the microcontroller and the radio being integrated onto a single chip. ZigBee has been designed as a low cost radio network technology and estimated cost to the manufacturer of adding ZigBee to a device is a under US\$2 per device [19].

When the IEEE 802.15.4a alternate physical is ratified it is expected that the ZigBee specification will be modified (if necessary) to make use of it's inherit advantages, such as precision ranging.

\subsubsection{Alternate Low Rate Wireless Personal Area Networks (IEEE 802.15.4a)}

The IEEE 802.15.4a task group was formed to provide a communications standard that provides high precision ranging capability (less than 1 meter accuracy) and high aggregate throughput as well as the already defined targets of IEEE 802.15.4. IEEE 802.15.4a is still under development and has not been ratified. Two optional physical layers have been confirmed, a chirp spread spectrum (CSS) based radio 
operating in the $2.4 \mathrm{GHz}$ band and a UWB impulse radio. Only the UWB physical implementation will provide the high accuracy ranging [20].

\subsubsection{Wireless Metropolitan Area Networks (IEEE 802.16)}

The IEEE 802.16 working group was established in 1999 to provide broadband wireless access as a "last mile" solution to the user. IEEE 802.16 standard was ratified in 2001 and specifies operations in the 2-66 $\mathrm{GHz}$ band. There are three separate physical layers that are designed to operate in different frequency bands within this range. With frequency bands of operation being so large data rates in excess of 120 Mbps are possible [21]. Worldwide Interoperability for Microwave Access (WiMax) is the certification mark for devices that pass the IEEE 802.16 standards. WiMax products are gradually becoming more common in the market today. As IEEE 802.16 is desired for high data rate long distance applications it is not suitable for implementing wireless sensor networks.

\subsubsection{Proprietary Protocols}

There are many proprietary radios available that are suitable for wireless sensor networks and don't fall within the IEEE 802 group of wireless standards. Chip manufacturers such as Nordic, Chipcon and Freescale produce various low power radios in various unlicensed bands. The risk of using proprietary radio standards to implement a wireless sensor network solution is that support and supply of radios can be limited as only one company produces them. The IEEE and other ratified standards are passed by members from many companies which ensure that the specification is robust and usually ensures that many vendor options are available.

\subsubsection{Wireless Protocol Summary}

While a lot of the above standards seem ideal for wireless sensor networks some are either incomplete or hardware is not currently available for them. A summary of the most viable wireless technologies for wireless sensor networks can be seen below in Table 2 . 


\begin{tabular}{|c|c|c|c|c|c|}
\hline Network Property & $\begin{array}{l}\text { IEEE } \\
\mathbf{8 0 2 . 1 1 b}\end{array}$ & \begin{tabular}{|l|} 
IEEE \\
$\mathbf{8 0 2 . 1 1 a}$
\end{tabular} & $\begin{array}{l}\text { IEEE } \\
\mathbf{8 0 2 . 1 1 g}\end{array}$ & $\begin{array}{l}\text { Bluetooth I } \\
\text { IEEE 802.15.1 }\end{array}$ & $\begin{array}{l}\text { ZigBee I } \\
\text { IEEE 802.15.4 }\end{array}$ \\
\hline Frequency Band & $2.4 \mathrm{GHz}$ & $5.8 \mathrm{GHz}$ & $2.4 \mathrm{GHz}$ & $2.4 \mathrm{GHz}$ & $2.4 \mathrm{GHz}$ \\
\hline Technology & DSSS & $\begin{array}{l}\text { OFDM / } \\
64 Q A M\end{array}$ & $\begin{array}{l}\text { OFDM / } \\
\text { DSSS }\end{array}$ & FHSS / GFSK & O-QPSK \\
\hline $\begin{array}{l}\text { Required System } \\
\text { Resources }\end{array}$ & $1 \mathrm{MB}+$ & $1 \mathrm{MB}+$ & $1 \mathrm{MB}+$ & $250 \mathrm{~KB}+$ & $4 \mathrm{~KB}-32 \mathrm{~KB}$ \\
\hline $\begin{array}{l}\text { Maximum Baseband } \\
\text { Throughput }\end{array}$ & $11 \mathrm{Mbps}$ & $54 \mathrm{Mbps}$ & $54 \mathrm{Mbps}$ & $723.2 \mathrm{kbps}$ & $250 \mathrm{kbps}$ \\
\hline Approximate Range & $\begin{array}{l}100 \\
\text { meters }\end{array}$ & $\begin{array}{l}100 \\
\text { meters }\end{array}$ & $\begin{array}{l}100 \\
\text { meters }\end{array}$ & $\begin{array}{l}100 \text { meters } \\
\text { (Class } 1)\end{array}$ & 75 meters \\
\hline Power Usage & Medium & Medium & Medium & Low & Very Low \\
\hline $\begin{array}{l}\text { Maximum Nodes per } \\
\text { network }\end{array}$ & $\begin{array}{l}254 \text { per } \\
\text { IP } \\
\text { subnet }\end{array}$ & $\begin{array}{l}254 \text { per } \\
\text { IP } \\
\text { subnet }\end{array}$ & $\begin{array}{l}254 \text { per } \\
\text { IP } \\
\text { subnet }\end{array}$ & $\begin{array}{l}8 \text { per Piconet } \\
\text { (Multiple } \\
\text { Piconets form a } \\
\text { Scatternet) }\end{array}$ & $\begin{array}{l}65536 \text { per } \\
\text { ZigBee } \\
\text { Network } \\
\text { (Coordinator) }\end{array}$ \\
\hline
\end{tabular}

Table 2: Wireless Protocol Summary $[1,3,9,16]$

All of these wireless technologies are currently available as development kits and OEM modules. For wireless sensor networks that need high data rates per node IEEE 802.11 based technologies best satisfy the requirements. Wireless sensor networks for sports monitoring applications typically require much lower data rates per node and require around 40 nodes per network with a battery life of around 10 hours. Wireless personal area technologies such as Bluetooth and IEEE 802.15.4 solutions make ideal technologies for wireless sensor networks.

\subsection{Biomonitoring and Sports Monitoring Review}

Sports technology is an expanding industry with increasing resources being pooled in technology research to help the athlete swim faster, jump higher or run that little bit faster. Biomonitoring of patients' vital signs can give an early warning detection of a life threatening event and can save lives. These monitoring platforms use different kinds of sensors and signal processing techniques to convert the sensor data into meaningful results that can be utilised by a physician or an athlete's coach. Delivering this information back to a central station allows near real time monitoring, data synchronisation of multiple sensor platforms and many other benefits. T. F. Budinger conducted a review of the benefits of biomonitoring with wireless communications [22]. He highlights many sensor techniques for patient monitoring that can be applied to sports monitoring applications. He suggests several wireless 
communication protocols that are useful for long range communications (eg WAP) and short range communications (Bluetooth and HomeRF).

Accelerometer based sensor platforms are a popular area of research in the field of sports science. A system for measuring physical activity has been outlined in [23] which classifies daily physical activity as a combination of five basic gaits, five primary postures, and five limb movements and transitions. These classifications of physical activity are made from a platform containing several accelerometer sensors placed on the subject's foot, thigh and chest. Combining this system with an appropriate wireless network and wide area network interface allows remote monitoring of the subjects activities. The SMART Sensor Project [24] defines a wireless sensor platform design that follows the OSI network model. It proposes using the Bluetooth or ZigBee standards as the physical radios for wireless communications. The wireless analysis system presented in [25] and [26] uses accelerometer sensor data gathered with a sensor platform to characterise multi-limb motion and analyse the gait of athletes in sporting applications. The data in both applications is transmitted back to a central PC for analysis using a Bluetooth cable replacement solution.

Bluetooth communications have also been utilised in rower monitoring [27]. Global Positioning System (GPS) radios are used to determine the acceleration of the boat and Bluetooth communications have been used to transmit the data to the shore. Bluetooth communications can help negate the significant multipath effects that can be experienced over a flat surface like water due to its frequency hopped nature.

Bluetooth communications are being used and evaluated in various sports and biomonitoring applications. If these applications are to be successfully scaled to large numbers of sensor nodes, techniques need to be implemented to increase the number of active devices participating in Bluetooth networks. If these techniques are not implemented, the applications that utilise Bluetooth communications will be limited to seven wireless sensors on the Bluetooth based wireless sensor network.

\subsection{Bluetooth Protocol Review}

Bluetooth has been designed for, and is traditionally used in, cable replacement applications. It operates using a master / slave system where one node acts as a master connecting to a number of slaves to form a Piconet. The master controls communications on the Piconet, allowing the slaves to communicate using a Time Division Duplex (TDD) scheme. A slave can only communicate on the Piconet once the master has sent it a data or a poll packet (POLL). The slaves on the Piconet are unaware of each other and can only transfer data to the master. Bluetooth Piconets are made up of a master and a maximum of seven active slaves. Multiple Piconets can be connected together using bridging nodes, which 
communicate and route data between each Piconet. These Bluetooth structures are called scatternets. Bridging nodes can either be a slave in both Piconets or a master in one Piconet and a slave in another [1]. Figure 2 below shows various Bluetooth network structures defined by the standard.

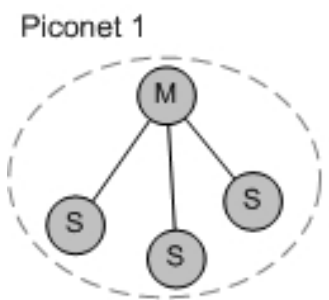

(a)

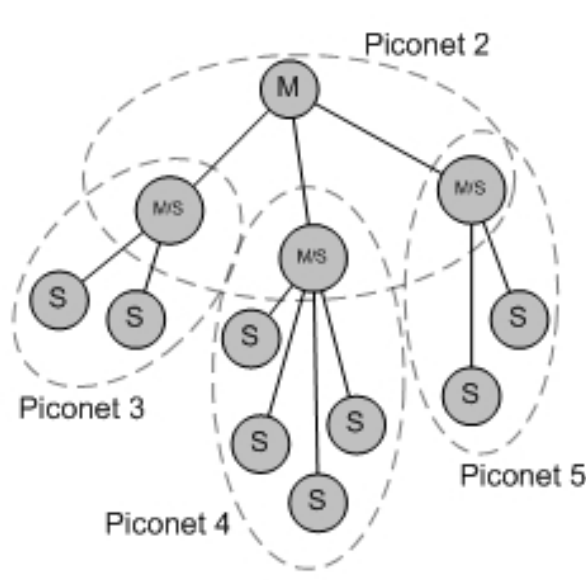

(b)

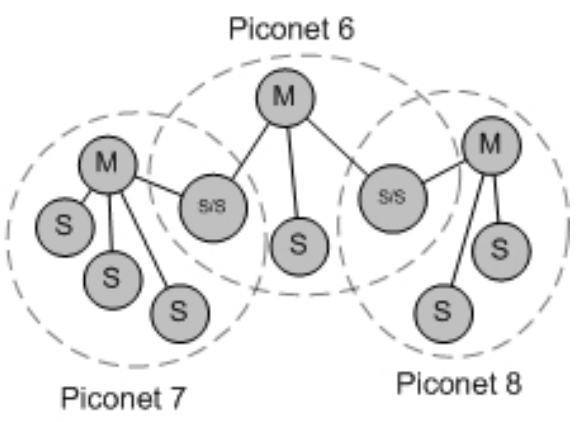

(c)

Figure 2: Bluetooth network structures;

(a) single Piconet structure, (b) master / slave bridging node Scatternet and (c) slave / slave bridging node Scatternet.

While Scatternets are described in the Bluetooth specification, however their implementation is not defined. As such Scatternet creation algorithms are an ongoing area of Bluetooth research. Many Bluetooth hardware manufacturers state that their products do not support Scatternet functionality.

Up to version 1.2 of the Bluetooth specification uses Gaussian frequency shift keying and can support an asymmetric data rate of $723.2 \mathrm{kbps}$ (using DH5 packets). Bluetooth version 2 supports Extended Data Rate (EDR) which uses a number of phase shift keyed based modulation schemes and can provide an asymmetric data rate of 2178.1 kbps (using 3-DH5 packets) [28]. Cambridge Silicon Radio (CSR) quotes that the maximum RFCOMM throughput for their Bluetooth hardware and stack implementation is approximately 380 kbps [29]. Figure 3 shows the theoretical baseband throughput per slave using DH5 packets and the RFCOMM throughput per slave based on a maximum throughput of $380 \mathrm{kbps}$. 


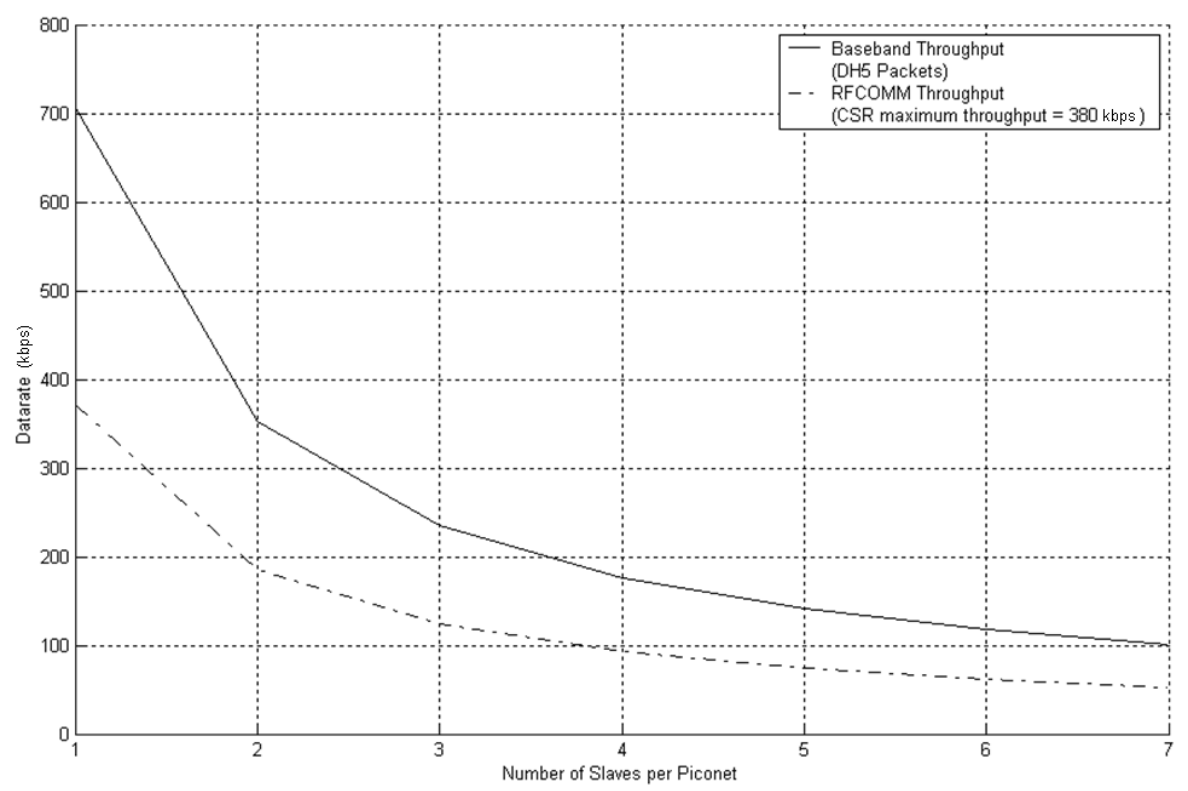

Figure 3: Bluetooth theoretical baseband and quoted RFCOMM throughput per slave [1,29].

There are three radio classes of Bluetooth devices each having a different maximum level of radiated power, and therefore can transmit over different path lengths. Bluetooth devices can also support a receive strength signal indicator (RSSI) measurement. This provides the node a means to automatically control the power level. Table 3 shows the three Bluetooth power classes.

\begin{tabular}{|l|l|r|}
$\begin{array}{l}\text { Bluetooth } \\
\text { Power Class }\end{array}$ & \multicolumn{1}{l}{$\begin{array}{l}\text { Radiated Power } \\
\text { Maximum }\end{array}$} & \multicolumn{1}{l|}{$\begin{array}{l}\text { Approximate } \\
\text { Range }\end{array}$} \\
\hline Class 1 & $100 \mathrm{~mW}(20 \mathrm{dBm})$ & 100 meters \\
\hline Class 2 & $2.5 \mathrm{~mW}(4 \mathrm{dBm})$ & 10 meters \\
\hline Class 3 & $1 \mathrm{~mW}(0 \mathrm{dBm})$ & 1 meters \\
\hline
\end{tabular}

Table 3: Bluetooth power classes [1].

Bluetooth uses the $2.4 \mathrm{GHz}$ unlicensed ISM band for transmission using the frequencies $2.4 \mathrm{GHz}$ to $2.4835 \mathrm{GHz}$. To coexist in this band Bluetooth uses a frequency hopped spread spectrum (FHSS) signal. The bandwidth is broken up into 79 channels, which the Piconet hops between at a rate of 1600 hops per second. The hopping sequence for each Piconet is different and is based upon the unique Bluetooth Address (BD_ADDR) of the master. If the hop sequence falls on an occupied hop channel, the data sent is lost and must be retransmitted. This means that the more interference from other wireless networks or other Bluetooth Piconets the lower the data rate. The current phase in the hopping sequence is defined by the master's onboard clock. Standard Bluetooth Piconet communications are further explained in Chapter 4.1 Standard Bluetooth Piconet Simulation of this document. 
As the Bluetooth communications channel is constantly frequency hopping, a master that wishes to discover and connect to a slave must find where the slave currently is in the hopping pattern. To do this the master frequency hops twice as fast and sends twice as many packets making it more likely that the slave will be listening on a given frequency channel when the master is transmitting. To discover Bluetooth devices in range the master performs an inquiry procedure. This is a lengthy process and can take up to 10.24 seconds to return a result (if the desired number of responses is not reached) [1]. Once a device knows the BD_ADDR of another device (either through the inquiry procedure or through user interaction) it can page the other device and form a connection. The device that forms the connection becomes the master of the Piconet. The connection is made by using the BD_ADDR of the slave device to calculate the page hopping sequence. It then estimates the current position in the hopping sequence and attempts to make a connection. The phase estimate of the hopping sequence can be completely wrong and can cause a delay in the connection of the two devices. However if the approximate phase is known, either from a recent inquiry or connection, then the connection process will be much faster. This inquiry process of device discovery requires the slave to be in an inquiry scan state listening for the master's inquiry message. Similarly the process of a device connection requires the slave to be in a page scan state listening for the master's page message.

Once connection has been established the Slaves are sent to one of four states; active, sniff, hold or park. These can be used to conserve the battery life of the node if communications can be reduced or suspended. In active mode the device communicates actively in the Piconet. Sniff mode reduces the amount of time the device is communicating in the Piconet. Hold mode suspends communication on the Piconet. Slaves in the previous three states are still considered one of the seven active slaves on the Piconet. When a device is in park mode data and voice communications are suspended on the Piconet and the device is no longer considered one of the seven active devices. In this manner more than eight devices can be part of a Bluetooth Piconet [1]. Further information regarding the Bluetooth sniff and park modes are given in Chapter 4.2.1 Piconet Sharing Bluetooth Network Structure Simulation and Chapter 4.2.2 Scatternet Tree Bluetooth Network Structure Simulation of this thesis.

The Bluetooth hardware and software used in this project conform to the Bluetooth version 1.1 Specification. During the project both the Bluetooth version 1.2 and 2.0 specifications were finalised by the Bluetooth SIG. The major improvements to the core Bluetooth functionality is adaptive frequency hopping (AFH) and faster connection times. Bluetooth ADF allows the radio to avoid channels that have been identified as occupied. This allows Bluetooth to coexist much better with IEEE $802.11 \mathrm{~b}$ and $802.11 \mathrm{~g}$ networks. The specification allows the number of hop channels used by the Bluetooth Piconet to be reduced from 79 to as low as 20 [30]. This allows the Piconet to avoid IEEE 802.11b and 802.11g channels which are $22 \mathrm{MHz}$ wide. Bluetooth version 2.0 introduced extended data rate (EDR) functionality. This uses phase shift keyed (PSK) based modulation schemes to provide a faster data rate - 18 - 
than standard Bluetooth communications. To preserve backwards compatibility with Bluetooth versions 1.1 and 1.2 the header of the EDR packets is modulated with GFSK and the data payload is modulated with the PSK modulation scheme [28]. All Bluetooth specifications are backwards compatible with previous versions of the specification. Figure 4 shows the Bluetooth stack as described in the Bluetooth specifications.

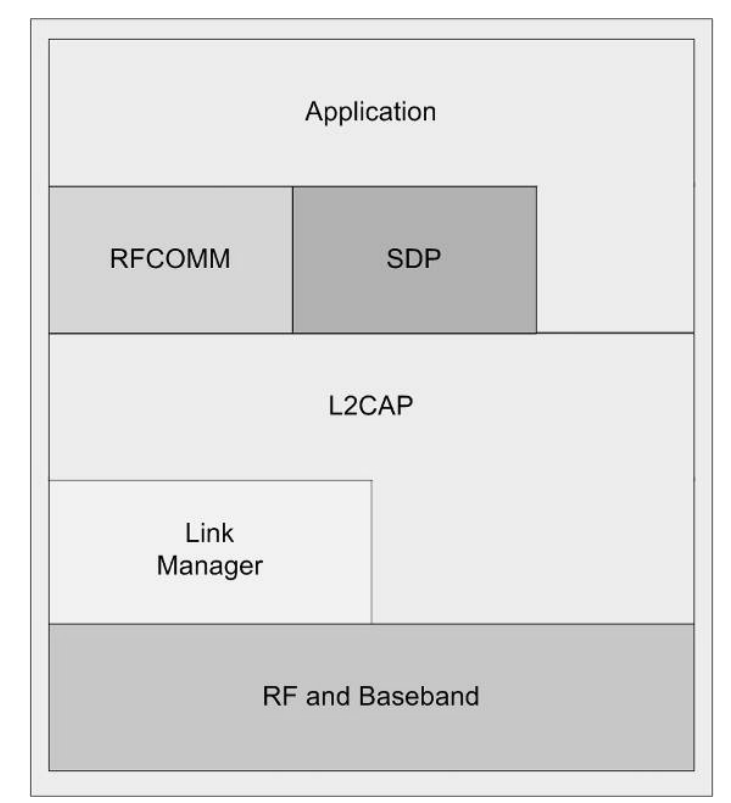

Figure 4: Bluetooth Stack as outlined by the Bluetooth Specification [1].

The Radio Frequency (RF) layer of the Bluetooth specification covers the physical $2.4 \mathrm{GHz}$ radio characteristics, the implementation of the modulation schemes and the hopping channel arrangement. The Baseband layer covers the majority of lower layer Bluetooth communications including frequency hop channel selection, communications channel control, network addressing, error correction and packet formats. The Bluetooth Link Manager Protocol (LMP) controls communications on the Piconet including the authentication, pairing, encryption, Baseband packet type and the switching of the four Bluetooth connection states. The Logical Link Control and Adaption Protocol (L2CAP) is responsible for the segmentation, reassembly and multiplexing of higher level data packets. It allows high level Bluetooth protocols to utilise much larger data packets than the Baseband packets provide and directs the incoming Baseband data to the correct upper layer application. The Service Discovery Protocol (SDP) provides the mechanism where Bluetooth devices can discover each other and the services that they are providing. Examples of Bluetooth SDP profiles that a device can support are Dial-up Networking Profile, Headset Profile, and Serial Port Profile (SPP). Conforming to specific Bluetooth SDP profiles ensures interoperability between Bluetooth devices from different manufacturers. The RFCOMM protocol provides serial port emulation over the L2CAP protocol. The SPP uses an RFCOMM based connection to provide 
a virtual serial port between two Bluetooth devices. RFCOMM / SPP based connections are being used in the wireless sensor network application as the sensor data is outputted by the wireless sensor platforms as a serial data stream. The Link Manager and the Baseband layers must also be used to change the Bluetooth connection states where required.

\subsection{Current Bluetooth Research}

The majority of research being conducted on the Bluetooth specification can be divided into several groups; inter-Piconet scheduling, application profile advancements, access point handoff schemes, Bluetooth positioning, Scatternet creation algorithms. Inter-Piconet scheduling deals with optimising communications within the Piconet, which optimises Piconet efficiency. As changing the existing Piconet scheduling modifies the Bluetooth specification, this research direction was not pursued. Only solutions that are achievable within the existing Bluetooth standards and Bluetooth hardware were considered. A lot of previous work has been undertaken into new application profiles and application profile development. The majority of this work does not relate to Bluetooth wireless sensor networks and has not been reviewed in depth in this thesis.

Bluetooth handoff schemes relate to devices being passed between Bluetooth access points with minimal loss of service. This is especially important in voice applications. If a sensor node loses network connection for an extended period of time the data buffers will overflow and data will be lost. Handoff schemes ensure that this loss of service period is as short as possible. A Bluetooth handoff scheme is presented in [31] for enhanced mobility between access points using Bluetooth based IP communications. This method of handoff between Bluetooth devices is the simplest with the disconnected node performing an inquiry for access points in range, connecting to the access point, preforming a master / slave swap and reconnecting the upper layer communications. This method requires the lengthy Bluetooth Inquiry procedure which delays the handoff between access points significantly. Experimental results from this research showed that Baseband layer handoff times of 5 seconds were possible using this method. Reconnecting the upper IP layer communications took significantly longer as the TCP/IP protocol employs an exponential back off on each unsuccessful retransmission event. Experimentally it took greater than 20 seconds for reconnection of upper layer communications to occur. This delay is unsuitable the intended wireless sensor network application. The hand off mechanisms proposed in [32] and [33] don't utilise the Bluetooth Inquiry procedure. Devices are handed off between Bluetooth access points by passing device information such as Bluetooth Addresses and timing information so that a device can be immediately Paged without having to perform the lengthy Inquiry discovery procedure. Simulation results in [33] show that a handoff time of less than $20 \mathrm{~ms}$ is possible with this scheme. The hand off scheme in [34] uses $\mathrm{HCl}$ buffer in Bluetooth devices to buffer the upper layer data so that the upper IP layers detect no loss of TCP/IP connection. This approach removes 
the large delays seen in [35] but can lead to buffer overflows and loss of data if the onboard buffer length is not large enough to accommodate the hand off delays. The Bluetooth Personal Area Network (PAN) working group developed the Personal Area Network Profile that specifies Ethernet Encapsulation, Single Piconet IP based networks, master data forwarding and Bluetooth network access points [36]. A future goal of the working group is to develop a specification for Bluetooth Access Point Roaming (APR). The PAN-APR profile is an extension on the PAN profile that specifies how Bluetooth access points perform handoff procedures. The final profile specification has not been released yet, but revolves around Bluetooth access points exchanging device addresses and timing information to allow Bluetooth inquiry procedures to be removed from the handoff process.

The Bluetooth specification includes a received signal strength indicator (RSSI) that can be used to approximate the distance between two Bluetooth devices. Bluetooth positioning takes the RSSI value, calculates the distance between devices and triangulates the device position [37]. By reducing the device power levels, approximate positioning can be achieved by which fixed Bluetooth access points are currently in range [38]. These projects have had limited success with Bluetooth tracking and have not produced definitive results.

Bluetooth Scatternets have attracted a lot of research as the Bluetooth specification fails to describe them adequately. A main area of research is Scatternet creation algorithms. Scatternets can either be formed using slave / slave bridging nodes, master / slave bridging nodes or a combination of both. This body of work focused on creating a Bluetooth wireless sensor network that requires all data to be collated in a central location. A tree structure built from master / slave bridging nodes best satisfied this and therefore Scatternet creation algorithms built around slave / slave bridging nodes [39, 40,41] were not considered for use in this implementation. The two phase Scatternet creation algorithm described in [42] suggested creating a control Scatternet for the routing of control information throughout the Scatternet and creating dedicated Piconet or Scatternets for the transmission of data between nodes. It was suggested that the control Scatternet could park the slaves connected so that more than eight devices could be connected on a single Piconet. This formation protocol required the continuous creation and destruction of Bluetooth networks which placed significant overheads on the network. It is best suited for ad hoc situations for data sent in bursts. The Bluenet Scatternet creation scheme uses a three phase procedure for Scatternet creation [43]. Network creation begins with all devices Inquiring to discover neighbouring devices. All devices then begin Page Scanning while Paging the discovered neighbouring devices. If a device is successfully connected to by another device it stops page scanning. The remaining devices that aren't connected in the first two stages then connect to their neighbours, forming the Scatternet bridging nodes. This procedure gives no control over the Scatternet formation, resulting in unpredictable network topologies. If there aren't enough remaining devices left after the second phase the Scatternet would be segmented. It is possible that devices remain unconnected after the Scatternet connection phase is over. 
There is also no control over the number Bluetooth Piconets formed. For the highest throughput this number should be kept as low as possible. The Bluetrees Scatternet creation algorithm [44] provides the best Scatternet based solution for wireless sensor networks. The Scatternet is created by a designated Blueroot node which connects to it's adjacent neighbours. These neighbours then connect to their unconnected neighbours forming a master / slave bridging node based Scatternet tree. Bluetrees aren't appropriate for most ad hoc network situations as the entire Scatternet network traffic must be routed through the Blueroot node. This makes the use of Bluetrees especially useful for wireless sensor networks as network route discovery is no longer required. Communications need only be past upwards through the Scatternet towards the Bluetooth access point acting as the Blueroot node.

The use of Bluetooth wireless technology is being considered by the IEEE P1451.5 Wireless Sensor Working Group as the solution for wireless industrial sensor networks [45]. The RF wireless technologies working group (P1073.0.1.1) of the IEEE 1073 Point of Care Medical Device Communication Industry Group has also been considering using Bluetooth as part of the technical report on RF wireless solutions for point to point medical communications [46]. As Bluetooth technology is becoming a popular choice in wireless sensor networks techniques are needed to increase the node limits in Bluetooth networks.

\subsection{Wireless Sensor Networks Review}

Much of the recent research in wireless sensor networks focuses on implementing large scale networks of Micro-sensors $[47,48,49]$. These wireless networks provide a low data rate, have a low transmission range and thousands of nodes can be implemented in a single network. The collected data from each node itself may not be accurate, but the combined data from a number of nodes can present an accurate representation of the monitored system. An example of micro-sensors is the mote platform originally developed at University of California, Berkeley (Berkeley UC) [50,51, 52]. These micro-sensor motes typically run a cut down operating system called TinyOS developed at Berkley [53]. Motes are typically used in large scale network deployments, which can have node numbers in the hundreds. Micro-sensor platforms are typically used in applications such as asset tracking and industrial monitoring.

The research presented in this thesis focused on implementing a wireless sensor network for macrosensors. Macro-sensors provide a higher data rate than micro-sensors, have a higher transmission range, lower battery life and are typically implemented in much smaller numbers. The targeted sensor platform was designed by the CRC for Microtechnology, the Center for Wireless Monitoring Applications and Griffith University [2]. These platforms are typically used in low numbers and typically used in sports monitoring applications for gathering athlete performance data. These macro-sensor platforms require a larger bandwidth than that available from the Berkeley motes and other micro-sensors. Table 4 shows a comparison of micro-sensors with higher bandwidth macro-sensors. 


\begin{tabular}{|l|l|l|}
\hline Network Property & Micro-sensors & Macro-sensors \\
\hline Number of nodes & Large sensor networks (up to thousands) & Small and medium sized networks \\
\hline Power Consumption & Very small (battery life measured in years) & Low to medium (battery life hours) \\
\hline Data Rate & Very low to low & Medium to high \\
\hline Communication Range & Low & Medium to high \\
\hline Network Connections & Multi-hop & Single and multi-hop \\
\hline
\end{tabular}

Table 4: Micro-sensors vs. macro-sensors.

Wireless sensor networks can route data back to the receiving unit via a number of other nodes (multihop network). If a routing node loses connection (either by moving out of range or temporarily becoming unreachable), the network must find a new path for the data to be transmitted. If nodes frequently lose connection more time is needed to establish new transmission paths. This causes transmission buffers to overflow and data to be lost. As the proposed network environment will suffer frequent temporary connection drop outs, it was important to minimise the time required to reconfigure the network. One method to achieve this was to limit the network to single hop connections only. While this meant fewer nodes can be connected to a fixed receiver, during a network dropout only the specific node is affected and therefore network reconfiguration is simple. When multi-hop connections were necessary, the maximum number of multi-hops to the receiver was limited by the network. This limitation reduced the complexity of network reconfiguration.

There are two main Bluetooth based hardware sensor platforms; the Intel Imote [54] and the BTnode developed by the Swiss Federal Institute of Technology (ETHZ) [55]. The BTnode version 2 consists of an Atmel ATMega 128 processor and an Ericsson Bluetooth module. Scatternet functionality was not supported by the Ericsson chipset. Version 3 of the BTnode changed the Bluetooth hardware to a Zeevo (bought by Broadcom in 2005) chipset that supports Scatternet capabilities. The Intel Imote features a Zeevo module that has an integrated ARM based processor, Bluetooth radio, SRAM and Flash memory. Both of the sensor platforms software was based upon the TinyOS operating system that is widely used in wireless sensor networks. These Bluetooth hardware sensor platforms have been used in many Bluetooth wireless sensor research projects.

The Bluetooth wireless sensor research presented in [56] used version 2 of the BTnode to test and evaluate Bluetooth wireless sensor networks. As the Ericsson chipset does not support Scatternet functionality, two Bluetooth physical layers were used to enable multi-hop networking topologies. The Scatternet creation algorithm used was based on the Bluetree creation algorithm [57]. Time division multiplexing was used at the application level to poll each of the sensor nodes for data. A Bluetooth based deployment support network was presented in [58]. This Bluetooth Scatternet was designed to provide a support network to wireless sensor network nodes allowing code testing and debug information 
to be received from the sensor nodes while being tested in a similar environment to the intended deployment. BTnode version 3 devices were used to form the support Scatternet. The Scatternet creation method is a basic inquire and connect style algorithm that creates a basic Master / Slave Scatternet tree. Scatternets of over 70 nodes have been demonstrated with this hardware.

\subsection{Wireless Technologies and Literature Review Summary}

The wireless technologies presented in this review have been included for completeness and provide an alternate to the mandated Bluetooth Specification based wireless sensor network. Several wireless biomonitoring and sports monitoring research projects have been reviewed that are using or evaluating the Bluetooth Specification. To allow Bluetooth based wireless sensor networks to monitor more than seven active sensor nodes at a time, additional Bluetooth network structures are required to be developed.

The review into existing relevant research involving the Bluetooth Specification presented existing Bluetooth handoff techniques and Scatternet creation algorithms. The proposed wireless sensor network Bluetooth structures build upon this research which is mainly simulation based. The major outcome of this body of work is to produce viable Bluetooth network structures that are able to be implemented with existing Bluetooth hardware. This added an additional constraint of hardware and software limitations to the design process. 


\section{Bluetooth Wireless Sensor Network Design}

The design of the Bluetooth wireless sensor network was spilt into two parts; the Bluetooth networks themselves and the overall sensor network topology. The overall sensor network topology includes Bluetooth access points, high speed data backbone and the central data repository. The network topology is explained including suitable high speed network types and hand off mechanisms for use between Bluetooth access points. Wireless sensor cells were created to assist in access point handoff. Four Bluetooth network types were proposed for Bluetooth based wireless sensor networks allow more than seven sensor nodes to exist in each wireless sensor cell. Piconet Sharing and Scatternet Tree Bluetooth Structures are based around nodes sharing the time they are active in the Piconet and Piconet Tree and Multiple Piconet Root Node Structures rely on nodes utilising multiple Bluetooth radios.

The Piconet Sharing, Piconet Tree and Multiple Piconet Root Node Structures have been developed in this body of work and represent novel techniques for extending the node limits in Bluetooth networks within the Bluetooth Specification. The Scatternet Tree Bluetooth Structure was developed based upon the work presented in [44].

The Bluetooth Network Structures were designed using the Bluetooth version 1.1 specification [1]. The network structures presented still apply to devices utilising additional features that comply with version 1.2 and version $2.0+$ EDR of the Bluetooth stack. With all current Bluetooth specifications the maximum number of active slaves in a Piconet is seven. This is too few for the desired sports monitoring wireless sensor application.

\subsection{Bluetooth Wireless Sensor Network Topology}

The Bluetooth wireless sensor network topology is built around a number of fixed Bluetooth access points networked using a traditional wired system (eg Ethernet), a high speed IP based powerline communications standard (eg HomePlug) or high speed IP based non interfering wireless communications (eg IEEE 802.11a). The Bluetooth access points collect the data from the Bluetooth network and collate the data at a centralised location for display and analysis. Each of the access points acts as a root node for the created Bluetooth network structure and controls the network handling the network initialisation, reconfiguration and data retrieval from the wireless nodes. Having a number of networked access points allows a larger range to be monitored and allows more wireless nodes to be connected to the wireless sensor network. Each of the Bluetooth networks has been limited to a single hop network, meaning that all wireless nodes are in range of the current Bluetooth access point. Ensuring that the access point is in range of all sensor nodes connected to the Bluetooth structures 
makes network configuration an easier task and simplifies handoff between each of the fixed root nodes. By using directional antennas and controlling the transmitted power of the Bluetooth access point, wireless cells can be created in the wireless sensor network. These wireless cells assist in hand off between the fixed root nodes and help to reduce radio interference with the adjacent access points in the wireless sensor network topology. Figure 5 shows a wireless sensor network made with multiple fixed root nodes each with a wireless cell.
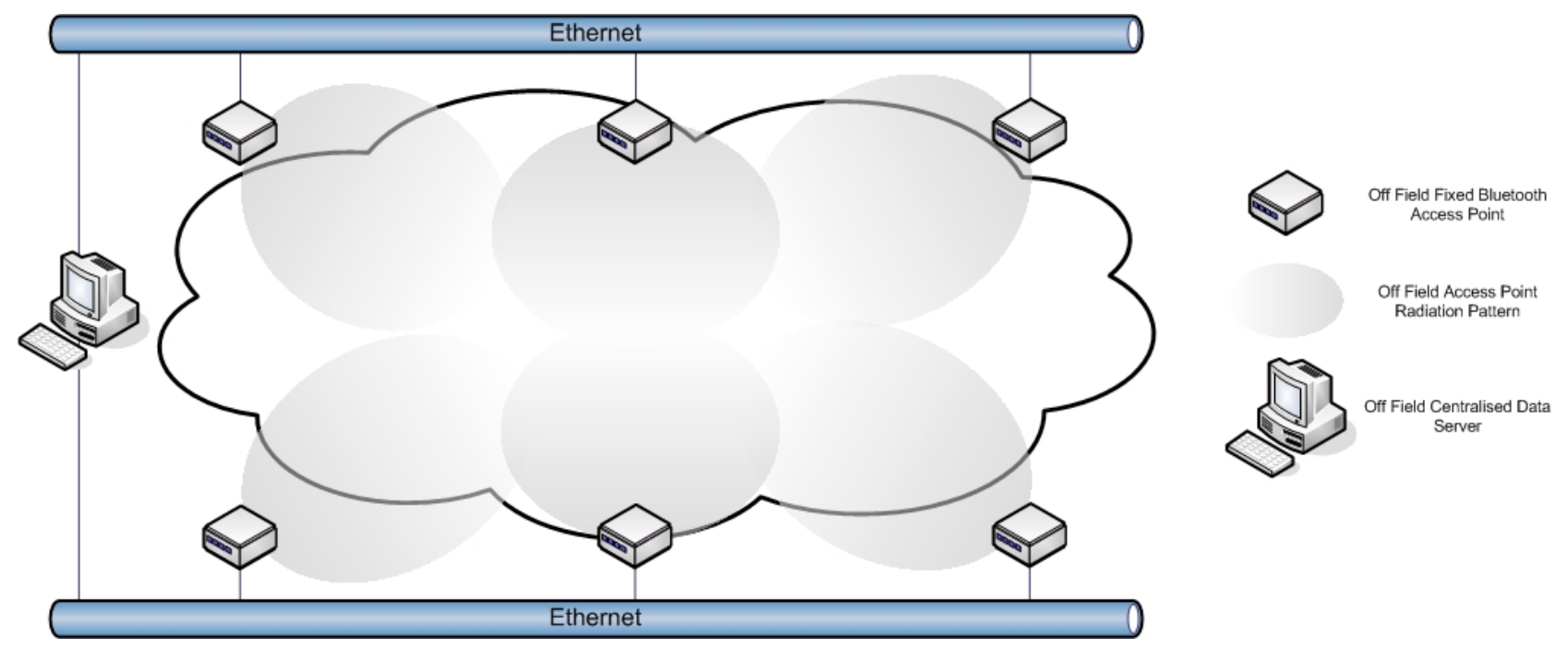

Figure 5: Wireless sensor network made up of wireless cells.

Due to the lengthy inquiry process in Bluetooth version 1.1 it is not practical to search for a device every time it needs to be connected. Constant network drop outs as the nodes move from one area to the next require a hand off procedure between the fixed root nodes. Passing mobile devices information between access points allows another access point to connect to a device without first having to perform the Bluetooth discovery process.

A soft handoff procedure occurs when the node doesn't loose a connection to the network at any time. For voice and media streaming applications this is essential and for data applications it is preferred. Soft handoff can be achieved using the RSSI value of the Bluetooth link. Once the RSSI falls below a preset threshold the mobile node is programmed to start page scanning awaiting a connection from other access points. The current access point instructs the surrounding access points to attempt to connect to the node by sending the mobile nodes BD_ADDR and phase information. Once another connection has been made to the mobile node, it can determine which signal is strongest (using RSSI) and disconnect the more distant access point. Once the hand off procedure is complete, the newly connected access 
point instructs the others to stop attempting to connect to the mobile device. This hand off procedure requires the mobile node to act as a slave in multiple Piconets, which requires Scatternet capabilities.

This soft handoff method can be implemented with a standard Bluetooth Piconet structure, however if a more complicated Bluetooth structure involving multiple Piconets is implemented then a soft hand-off procedure may not be possible. Complex Bluetooth structures put a major strain on the limited resources of the mobile nodes with many nodes already acting as M/S bridges, $\mathrm{S} / \mathrm{S}$ bridges or time sharing their active status in Piconets. An alternative to a soft hand off procedure is a hard hand off procedure where the root nodes only attempt to connect to the mobile node once it has disconnected from the network. This means that the mobile node will be disconnected from the network until reconnection is achieved. As long as the node becomes reconnected in a relatively short period of time no data will be lost. A future goal of the Bluetooth Personal Area Network (PAN) working group is develop a specification for Bluetooth Access Point Roaming (APR) [59]. The PAN-APR profile is still in the draft stages but is reported to specify handoff mechanisms between Bluetooth access points similar to those described above. The hand off mechanisms centre around passing the Bluetooth address and phase information between Bluetooth access points.

\subsection{Bluetooth Wireless Sensor Network Structures}

The proposed Bluetooth network structures exist within each of the wireless sensor cells created by the Bluetooth access points. Each Bluetooth access point is the Bluetooth structure's root node to the high speed backbone and the central data server. Each proposed Bluetooth network structure is explained with benefits and limitations highlighted. These network structures are based on version 1.1 of the Bluetooth specification, but are relevant and compatible with the later versions of the Bluetooth specification. The Bluetooth access points act as the master of each Bluetooth network structure connecting to each of the wireless nodes in turn. The Bluetooth access point can either receive device information from the high speed backbone (either the central data sever or another access point) or can perform periodic inquiries to locate unconnected nodes. A summary of the proposed Bluetooth network structures is then presented to determine which best suit the wireless sensor network application.

\subsubsection{Standard Piconet Bluetooth Network Structure}

Bluetooth Piconets can have a maximum of seven active slaves on the network. Using standard Bluetooth Piconets for each wireless cell limits the number of wireless sensor nodes per cell to seven. Figure 6 shows two standard Bluetooth Piconets; a point to point link with one active slave and a point to multipoint link with seven active slaves. 


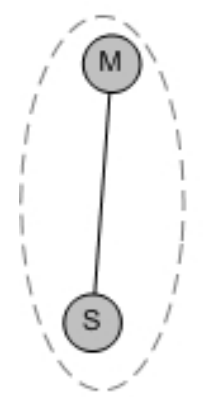

(a)

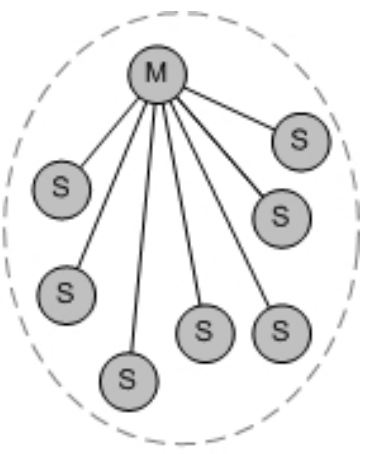

(b)

Figure 6: Standard Bluetooth Piconets;

(a) Point to Point Connection and (b) Point to Multi-Point Connection

If a slave temporarily loses connection to an access point or moves from one wireless cell to another either a soft or hard Bluetooth handoff procedure can be performed. The state diagram for the master of a standard Bluetooth Piconet appears below in Figure 7.

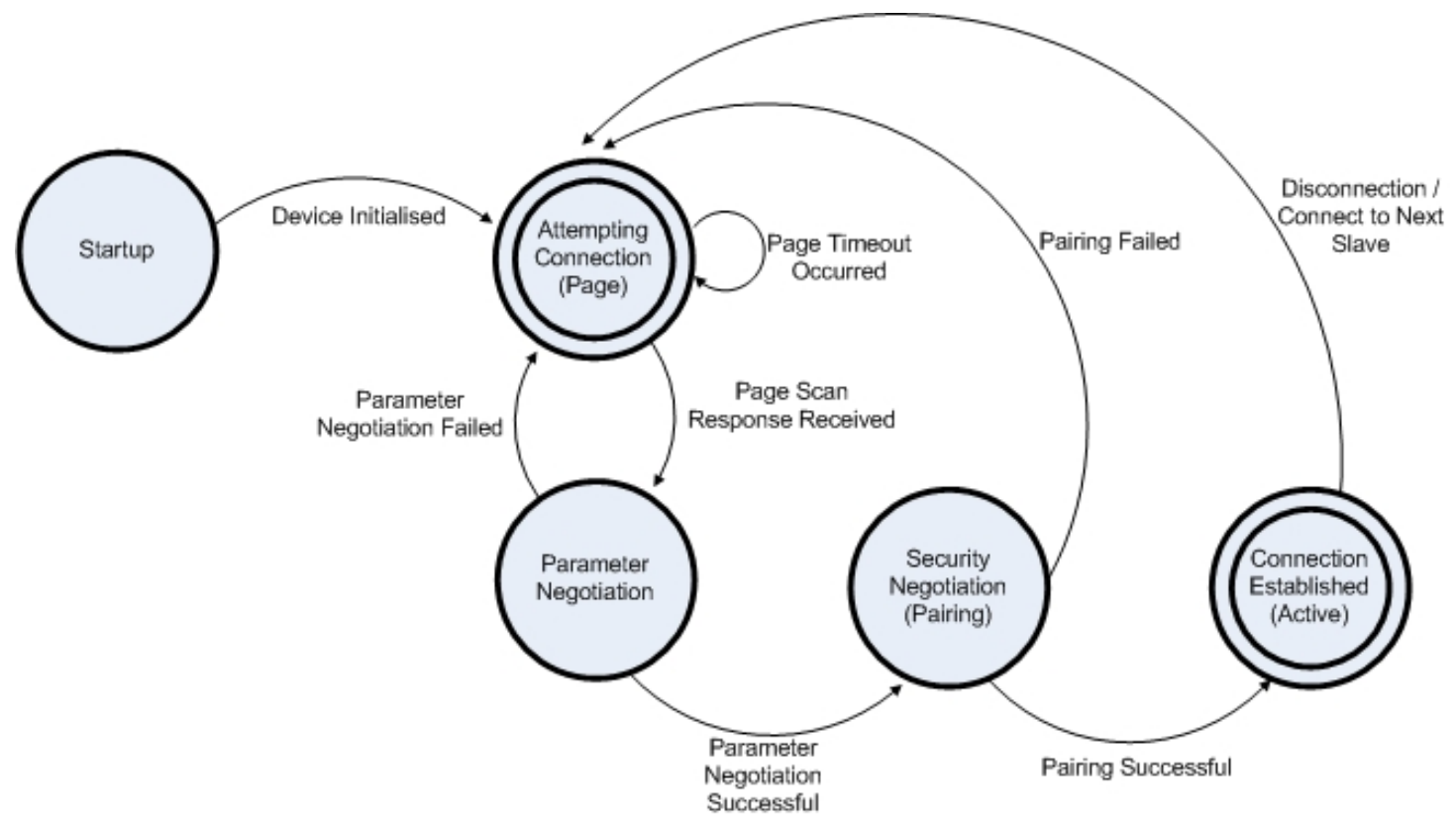

Figure 7: Standard Bluetooth Piconet Master State Diagram

Wireless slave nodes simply await connection from the Bluetooth access point by entering the page scan state. Once a successful connection has been achieved they begin transmitting their sensor data back to the access point. When the device is disconnected from the network, it returns to the page scan state awaiting reconnection from either the same access point, or another access point. Figure 8 is the state diagram of a standard Bluetooth Piconet slave. 


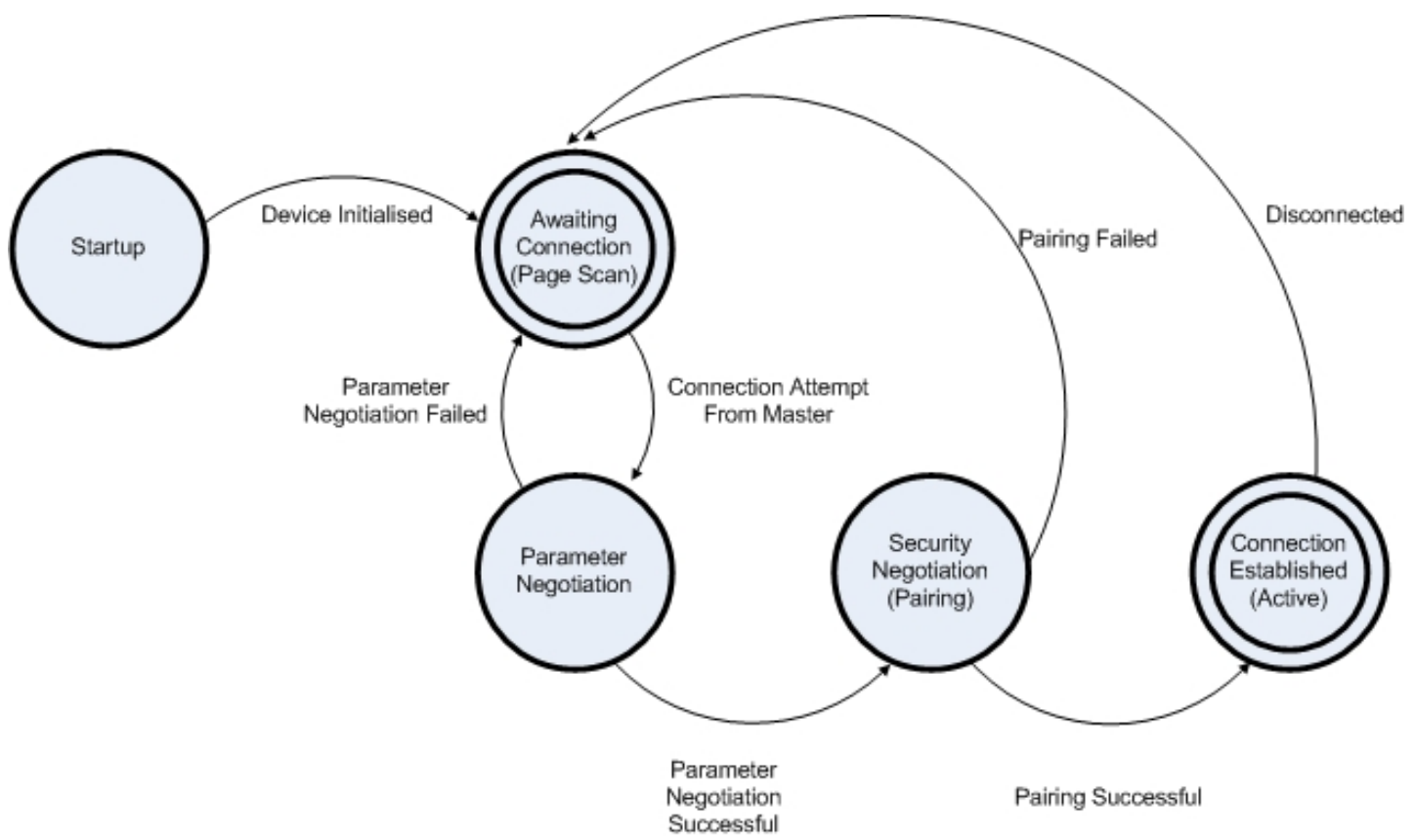

Figure 8: Standard Bluetooth Piconet Slave State Diagram

This Bluetooth network structure is the simplest and is the best choice for wireless sensor networks that only require a few nodes or where node density is low. More than seven wireless sensor nodes can be monitored by adding additional Bluetooth access points to the Bluetooth wireless sensor network. Bluetooth network structures that connect directly to the access point require the least network maintenance when network drop outs occur. This makes the Standard Bluetooth Piconet Structure suitable for environments where network drop outs are frequent.

A standard Bluetooth Piconet is only suitable for wireless sensing applications where less than seven sensor platforms need to monitored in a given area. This number can be extended by using multiple Bluetooth Access Points. The problem when only using this approach is when monitoring mobile devices (such in most sport monitoring applications) node densities may grow larger than the number of free Bluetooth AM_ADDRs. This results in unconnected wireless sensor platforms.

\subsubsection{Piconet Sharing Bluetooth Network Structure}

Bluetooth Piconets can contain only seven active devices because there is a maximum of seven active member address (AM_ADDR) per Piconet [1]. However when a connected device enters the park state it gives up it's AM_ADDR while still remaining synchronized to the piconet's frequency hopping pattern. This enables the slave to become active on the Piconet again relatively quickly and resume transmitting data. The Piconet Sharing Network Structure operates by sharing the seven AM_ADDRs between the slaves connected on the Piconet using the Bluetooth park mode. By rotating the connected slave devices 
between active and park states in groups, the TDMA communications on the Piconet is extended beyond the maximum seven active devices. However each device is limited to burst transmissions while it is active. Figure 9 is an example of the Piconet Sharing Network Structure using seven devices per Piconet Sharing Group and using two Piconet Sharing Groups. This configuration allows a maximum of fourteen devices to communicate per Bluetooth access point. The solid lines in the figure represent an active Bluetooth connection and the dashed lines represent a device in the park state.

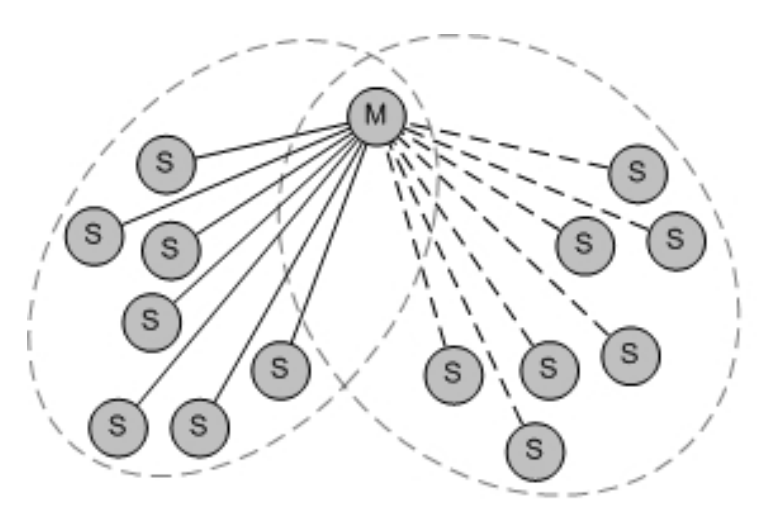

(a)

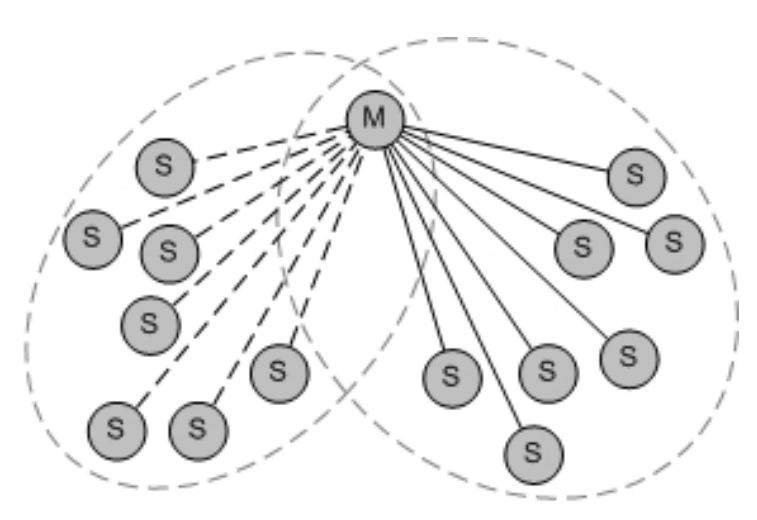

(b)

Figure 9: Piconet Sharing Bluetooth Network Structure;

(a) Group 1 is Active and Group 2 is Parked and (b) Group 2 is Active and Group 1 is Parked.

Piconet Sharing is controlled by the access point which acts as the master device for the Bluetooth Structure. The master connects to each slave and then parks the device. Once all devices are connected and in the park state the master periodically unparks each Piconet sharing group for a preset time. A state diagram of a Piconet Sharing Structure master is shown below in Figure 10. 


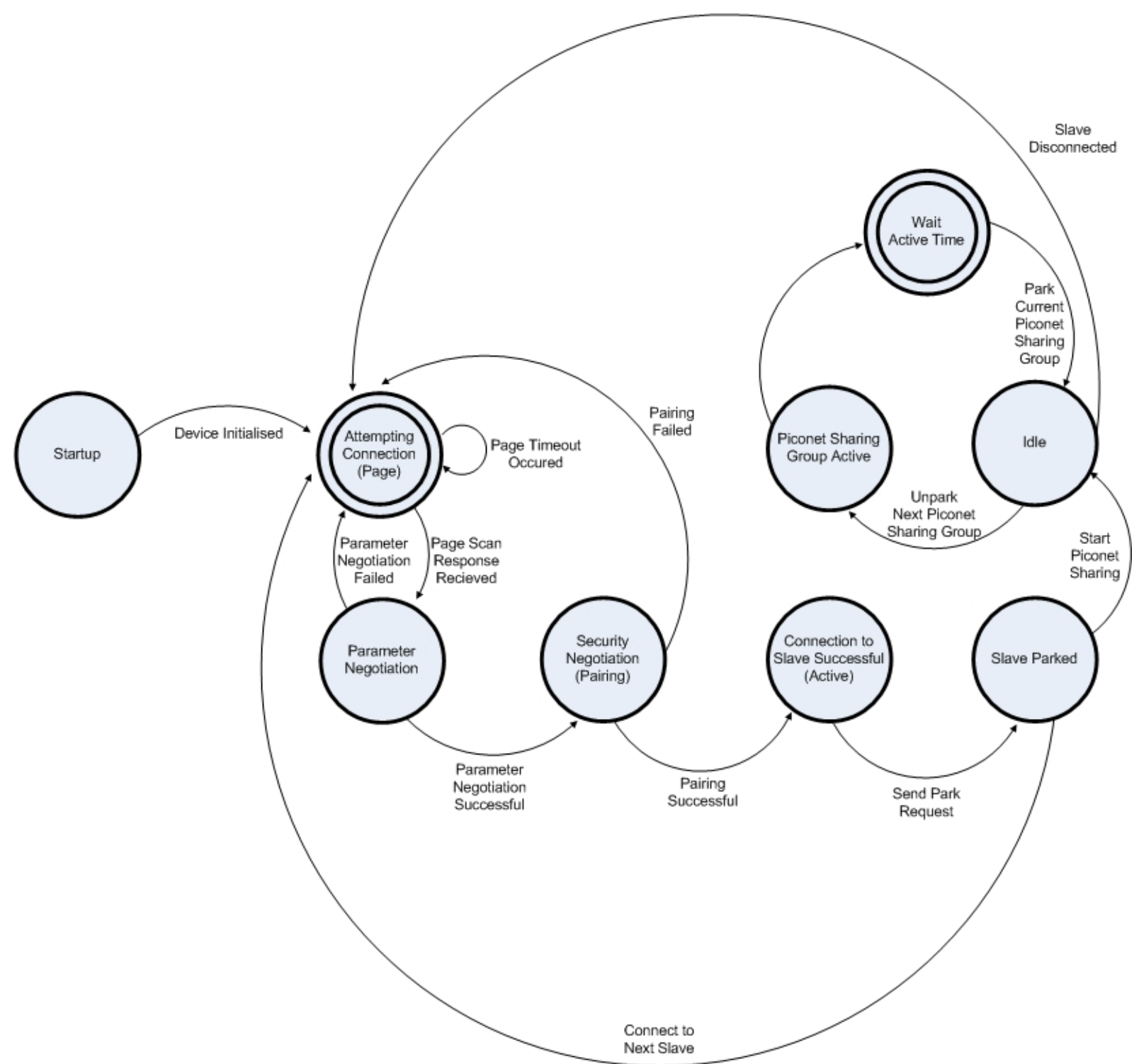

Figure 10: Bluetooth Piconet Sharing Master State Diagram

Wireless sensor nodes in the Piconet Sharing Network Structure only require standard Bluetooth slave functionality with the park low power state enabled. Figure 11 is the state diagram for the slave sensor node in the Piconet Sharing Network Structure. 


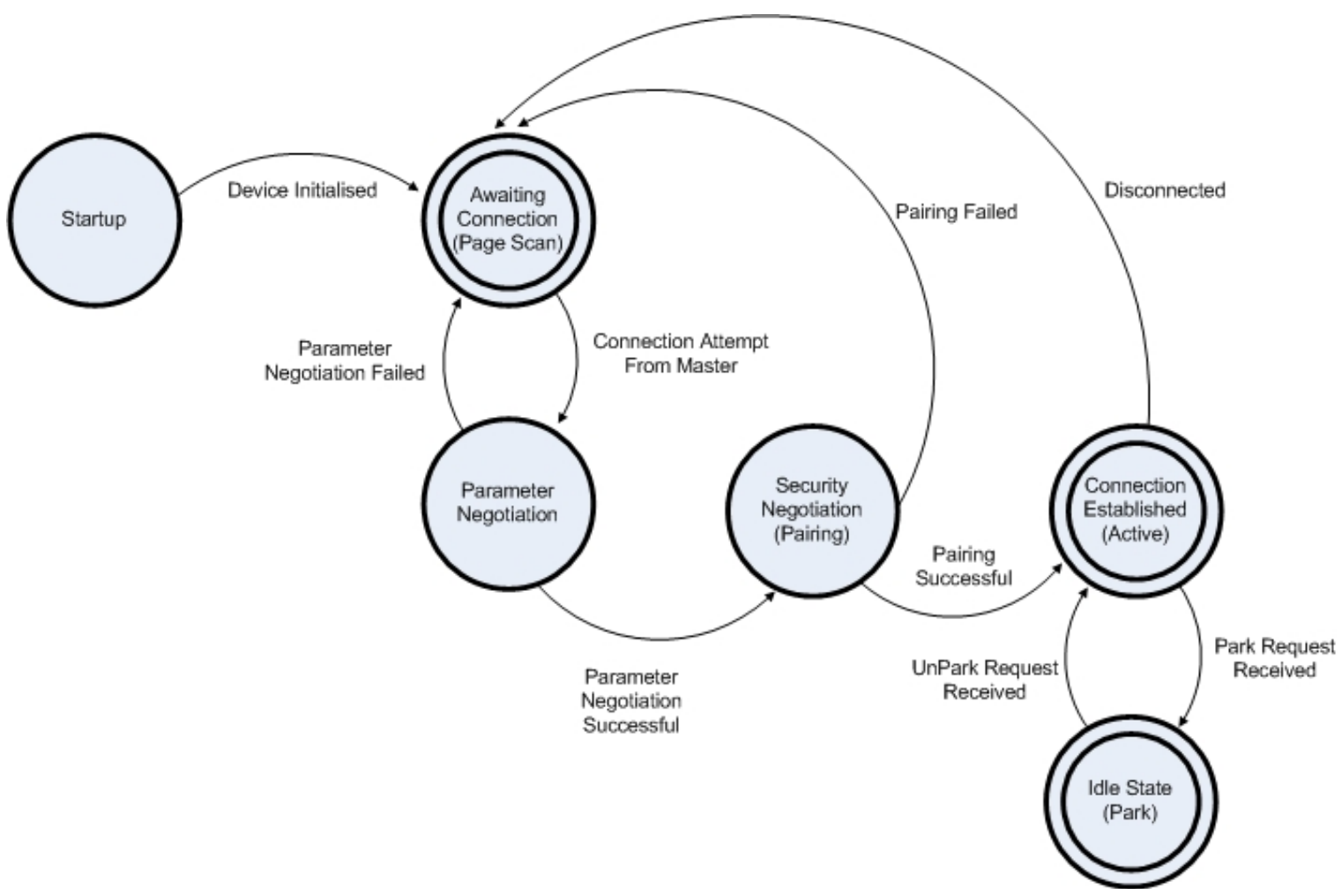

Figure 11: Bluetooth Piconet Sharing Slave State Diagram

In a high impact environment with mobile wireless sensor nodes the wireless network must be able to handle frequent drop outs and subsequently reconnect to the affected nodes. As each node in the Piconet Sharing Network Structure is only responsible for its own data, node reconnection is relatively trivial. As it is necessary to reconnect to disconnected nodes, the number of active slaves must always be less than the maximum of seven. This will ensure that there is at least one free AM_ADDR available to allocate to the disconnected node. Less than seven active slaves also allows sensor nodes that are approaching their data buffer limit to request to be unparked to transmit their data.

The wireless sensor nodes are swapped from being active to the park state in groups. The major disadvantage of Piconet sharing is that transmission in the network is burst data and there is a significant latency time between bursts. As the slaves can't communicate while parked they need to wait their turn for their group to become active. If the groups are configured to remain active for a large amount of time, parked nodes must be able to buffer the data collected from the sensor platform. An advantage of the Bluetooth Piconet Sharing Network Structure is that a Piconet Sharing enabled access point can connect to standard Bluetooth devices (that have park enabled) with no extra software required on the mobile wireless device. 
The Piconet Sharing network structure utilises a single hop radio link and is therefore suitable for wireless sensing applications that suffer frequent node drop outs (eg high impact sports monitoring). The wireless sensor nodes in the Piconet Sharing network structure suffer large latencies between transmission periods. This structure is only suitable for wireless sensing applications where the wireless sensor platforms are able to sufficiently buffer the data between transmissions.

\subsubsection{Scatternet Tree Bluetooth Network Structure}

A Bluetooth Scatternet is formed by nodes that are sharing their time between two different Piconets [1]. These devices become bridging nodes allowing data to be communicated between Piconets, allowing data transfer on the Scatternet. There are two types of bridging nodes in a Bluetooth Scatternet; master / slave bridging nodes (M/S) and slave / slave (S/S) bridging nodes. An Ad hoc Scatternet for evenly distributed data transfer among the nodes is usually formed using a combination of $M / S$ and $S / S$ bridging nodes. A large number of network topologies can be created using both types of bridging nodes and can be tailored to specific applications. Wireless sensor networks are designed to collect data from various wireless sensor nodes and transmit the information to a centralised server for analysis and storage. Creating a Scatternet structure from M/S bridging nodes alone allows a tree structure to be formed with the root node becoming the destination of all data on the network. This makes routing the sensor data through the network a simple task of repeatedly passing the data packets upwards through the Scatternet tree. For this reason the Scatternet used in this work used M/S bridging nodes only.

The Scatternet Tree Network Structure is created by the Bluetooth access point acting as a root node connecting to a number of wireless sensor nodes, and then instructing these nodes to connect to the remaining unconnected nodes. In doing this the root node creates a Scatternet using M/S bridging nodes. The root node controls the creation of the Scatternet and can manipulate the Scatternet using command messages passed down the Scatternet tree. After all nodes in the Scatternet are connected the $\mathrm{M} / \mathrm{S}$ bridging nodes should be placed into the Bluetooth sniff state. This ensures that the time the bridging node spends in each Piconet is shared correctly and network throughput is optimum. Figure 12 is an example a Scatternet Tree Network Structure. 


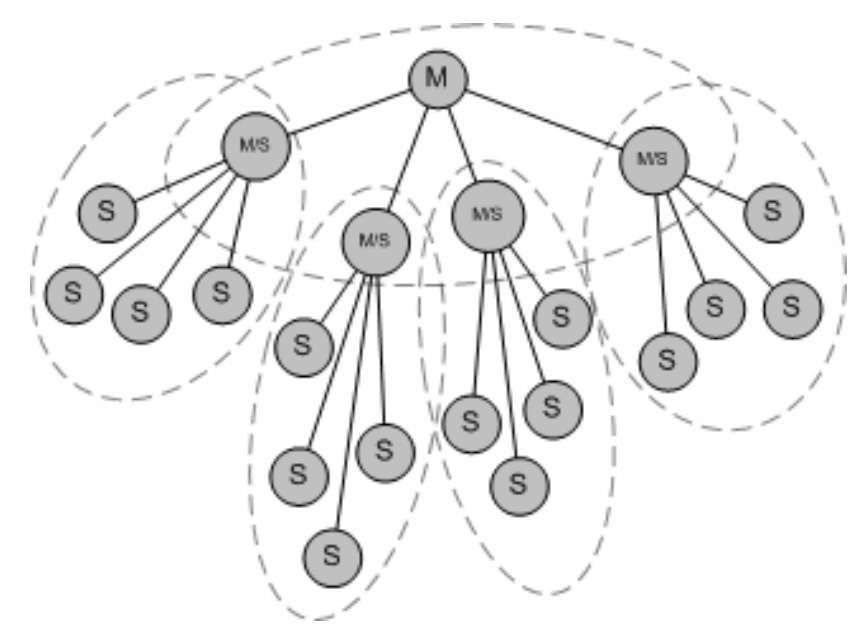

Figure 12: Scatternet Tree Bluetooth Network Structure.

The Bluetooth access point controls the Bluetooth Scatternet Tree telling each device to connect or disconnect from other nodes as needed. For a device to be added to the Scatternet tree it needs to be in range of the root node so it can be successfully discovered (if using an inquiry discovery method) or connected to (if using a Bluetooth access point hand off scheme). The Bluetooth access point itself is a standard Bluetooth device that passes application level Scatternet commands to the nodes in the Scatternet tree. The state diagram of a standard Bluetooth Piconet master was previously illustrated in Figure 7.

Each mobile sensor node has the flexibility to be configured either as a bridging node or slave depending on how the network is structured. The access point root node controls the Scatternet structure by telling each device to connect or disconnect from the Scatternet. This allows the network structuring to be handled by the root node which typically has a much higher processing power and greater knowledge of the current tree network structure. The Scatternet Bluetooth nodes are a combination of Bluetooth master and slave devices and function as shown in the state diagram shown in Figure 13. 


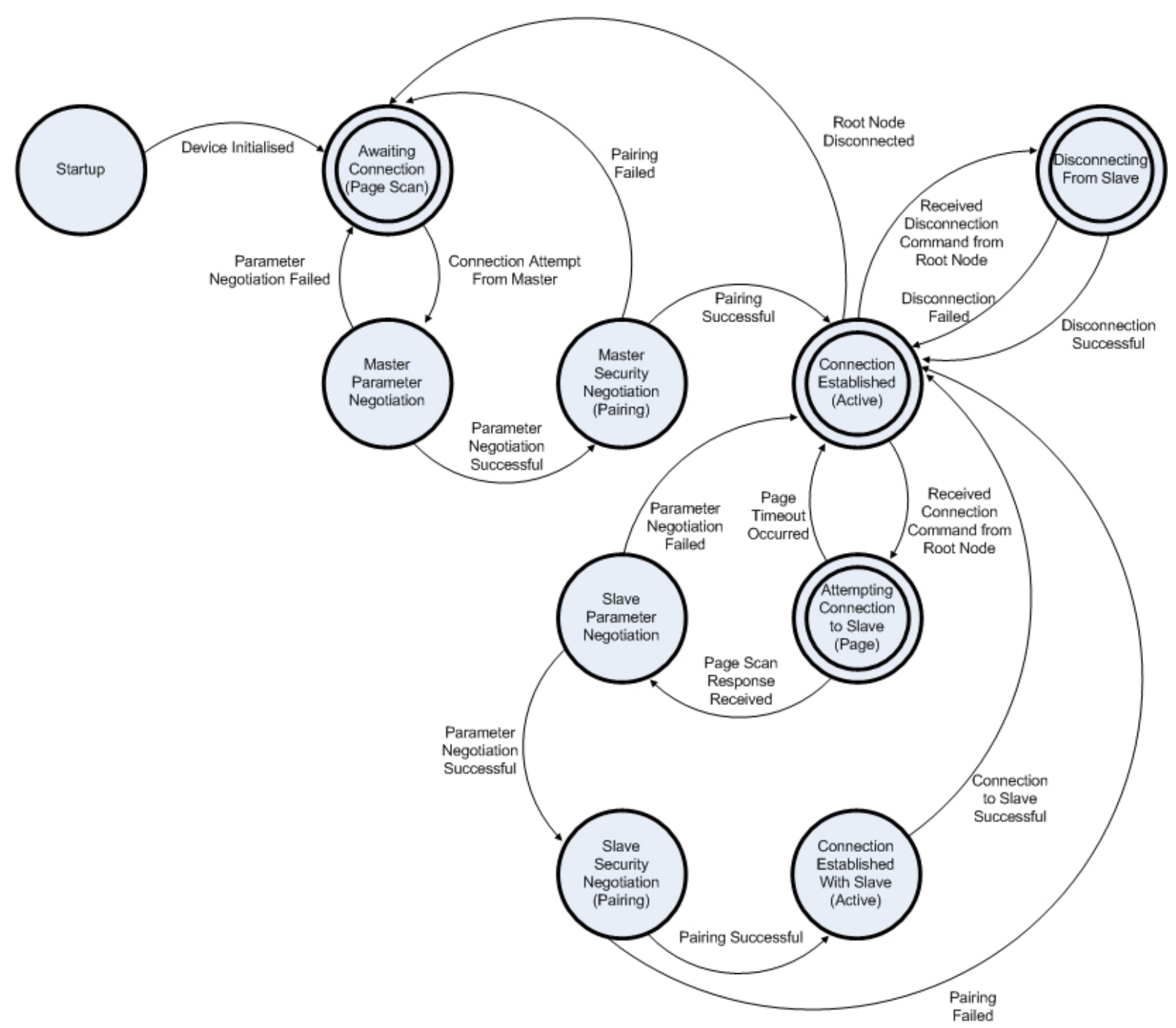

Figure 13: Bluetooth Scatternet Tree Node State Diagram

Using a single Bluetooth radio per mobile node is computationally intensive (as the nodes have limited resources) and limits the possible throughput. If a connection is lost between a bridging node and the root node, the slaves of the bridging node also lose their network path to the root node. For this reason Scatternet structures are not suitable for an environment where frequent drop outs occur, because of the large amount of network reconfiguration required. The advantage of this network structure is that as each device is constantly active on the Scatternet, the data is received as close to real time as possible.

The Scatternet Tree network structure is most appropriate for wireless sensing applications that only suffer infrequent node drop outs. This is due to the relatively complex task of restructuring the tree network. 


\subsubsection{Piconet Tree Bluetooth Network Structure}

Using multiple Bluetooth radios for bridging nodes allows a tree structure to be created, without the need for a Bluetooth radio be a member of multiple Piconets. The Piconet Tree Structure is made up of three types of nodes; the root node, bridging nodes and sensor nodes. The root node is a single board computer with Bluetooth radio acting as a master to connect to the bridging nodes. The bridging nodes consist of two Bluetooth radios and route data between the sensor nodes and the root node. The bridging nodes cannot collect data from a microsensor platform. Sensor nodes consist of a microsensor platform and a Bluetooth radio to send the collected data to the routing nodes. Figure 14 is an example of a Piconet Tree Network Structure.

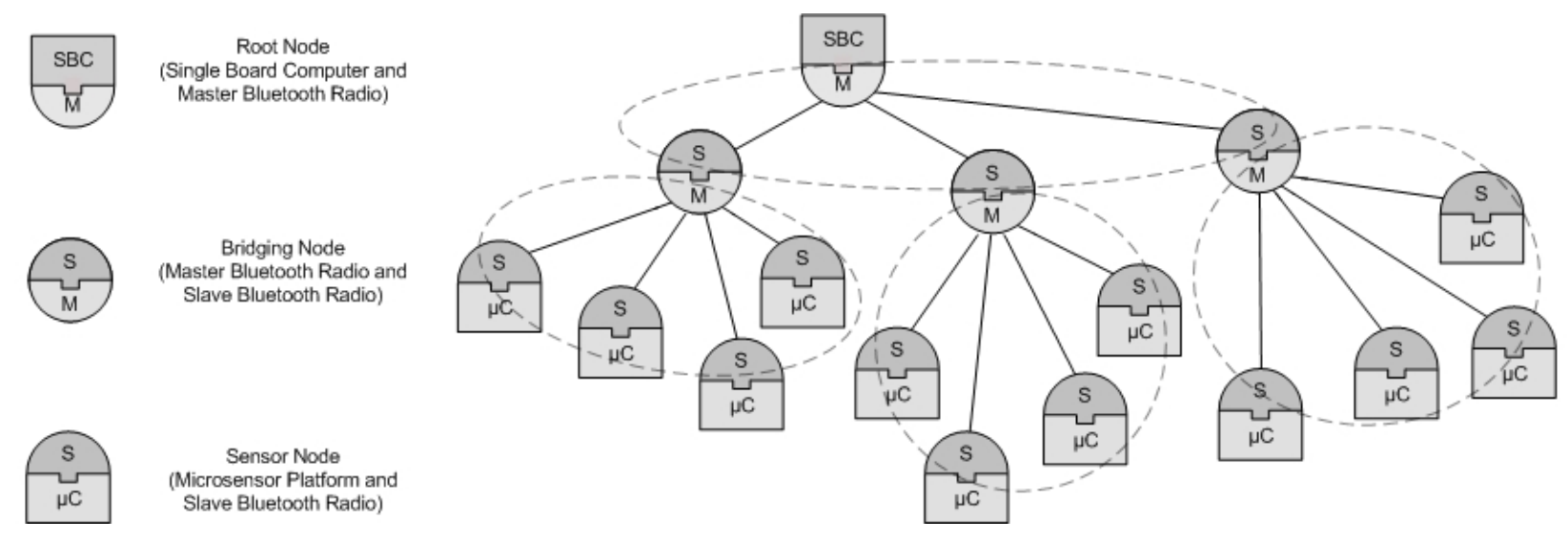

Figure 14: Piconet Tree Bluetooth Network Structure.

This Piconet Tree Structure can support a higher throughput than the Scatternet Tree Structure as it is less resource intensive for each Bluetooth device. It is created and maintained via the same method as the Scatternet Tree Structure, with the root node sending commands to each of the bridging nodes. All Bluetooth devices in the Bluetooth Piconet Tree Network Structure are standard Bluetooth devices. The bridging nodes in the network structure will have a much smaller battery life than the other nodes as they contain two Bluetooth radios. As only standard Bluetooth Piconets are used in this structure, standard Bluetooth power saving methods can be easily implemented. For example Bluetooth devices would remain parked and only unpark themselves when there is data to be sent. State diagrams of standard Bluetooth master and slave devices are shown in Figure 7 and Figure 8 respectively.

Due to the multi-hop nature of the Piconet Tree network structure it is best suited to applications that have a low instances of network drop outs. This Bluetooth structure is useful when wireless sensor nodes remain grouped together, as is the case when multiple sensors are attached to a single object or athlete. 


\subsubsection{Multiple Piconet Bluetooth Network Structure}

By adding multiple Bluetooth radios to the Bluetooth Access Point the number of Bluetooth sensor nodes per wireless cell can be extended beyond the Bluetooth maximum. Multiple Bluetooth radios allow the access point to create multiple Bluetooth Piconets, each having up to a seven active slaves at a time. Figure 15 shows the multiple radio root node.

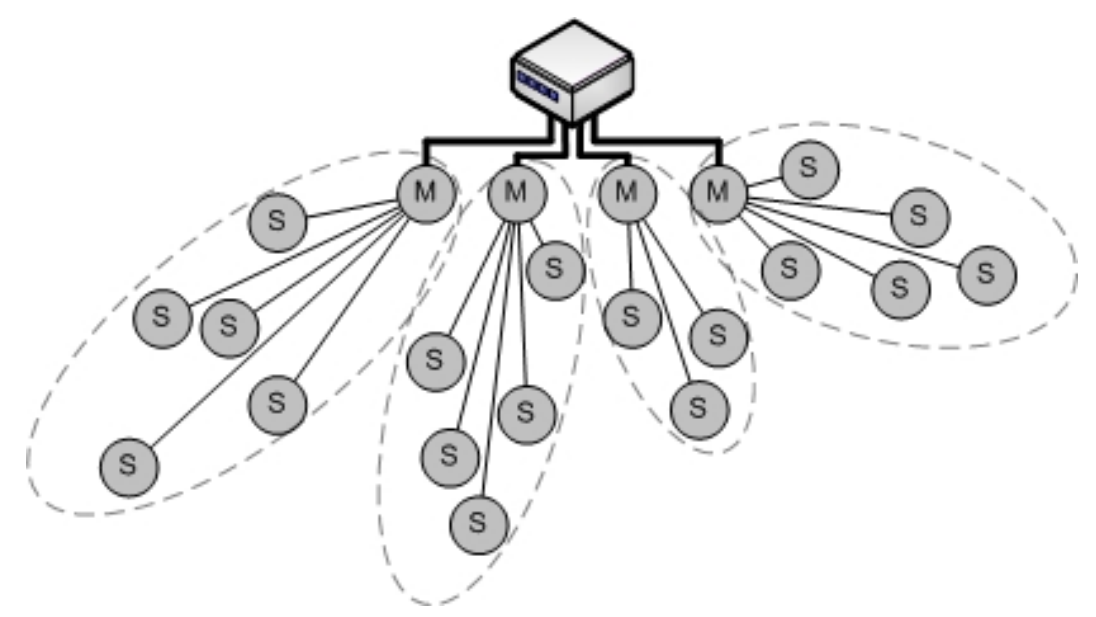

Figure 15: Multiple Piconet Bluetooth Network Structure.

All Bluetooth devices in this structure are standard Bluetooth devices and their state diagrams appear in Figure 7 and Figure 8. As the access point contains multiple radios, interference between Piconets will have a measurable effect on each Piconets throughput. This effect has been modelled in Chapter 4.1.2 Bluetooth Piconet Coexistence Simulation. To conserve the device's battery life slaves in this structure remain parked until they are required to transmit data. Some Bluetooth stack implementations only support a single Bluetooth radio per device. In this scenario the upper layers of the Bluetooth stack can be implemented externally in an embedded microcontroller and controlled via serial commands. This method could incur a Piconet throughput limitation due to the limitations of the microcontroller used.

The Multiple Piconet Bluetooth network structure has the potential to be the best overall solution for wireless sensor network sports monitoring applications. It only relies on a single hop wireless link and therefore node dropouts are easily handled. Creating a multiple Piconets in the same spatial area creates the opportunity for interference between Piconets. The number of Bluetooth radios added to the root node must be chosen to keep network interference to a minimum and not decrease the entire network structures throughput..

However the Bluetooth Access Point used must be modified to include the additional Bluetooth radios. Depending on the operating system and Bluetooth stack used for the access point this could be a 
complex task. The modification of the Bluetooth Access Point stack architecture is discussed further in chapter 5.2.4 Multiple Piconet Bluetooth Network Structure Testing.

\subsection{Bluetooth Wireless Sensor Network Design Summary}

A comparison of the described Bluetooth Network Structures is given in Table 5. Throughput for each of the proposed network structures was compared using the Bluetooth Simulation tools presented in Chapter 4 Bluetooth Wireless Sensor Network Simulation.

\begin{tabular}{|c|c|c|c|c|c|c|}
\hline Network Property & $\begin{array}{l}\text { Standard } \\
\text { Bluetooth } \\
\text { Piconet } \\
\text { Network } \\
\text { Structure }\end{array}$ & $\begin{array}{l}\text { Piconet } \\
\text { Sharing } \\
\text { Bluetooth } \\
\text { Network } \\
\text { Structure }\end{array}$ & $\begin{array}{l}\text { Scatternet } \\
\text { Tree } \\
\text { Bluetooth } \\
\text { Network } \\
\text { Structure }\end{array}$ & $\begin{array}{l}\text { Piconet Tree } \\
\text { Bluetooth } \\
\text { Network } \\
\text { Structure }\end{array}$ & $\begin{array}{l}\text { Multiple } \\
\text { Piconet } \\
\text { Bluetooth } \\
\text { Network } \\
\text { Structure }\end{array}$ & $\begin{array}{l}\text { Requirement } \\
\text { for Wireless } \\
\text { Sensor } \\
\text { Network }\end{array}$ \\
\hline $\begin{array}{l}\text { Maximum Sensing } \\
\text { Nodes Per Network } \\
\text { Structure }\end{array}$ & 7 & $\begin{array}{l}\text { Limited only } \\
\text { by Required } \\
\text { Throughput }\end{array}$ & $\begin{array}{l}56 \text { (Two Tree } \\
\text { levels) }\end{array}$ & $\begin{array}{l}49 \text { (Two Tree } \\
\text { levels) }\end{array}$ & $\begin{array}{l}28 \text { (Access Point } \\
\text { Using } 4 \\
\text { Bluetooth } \\
\text { Radios) }\end{array}$ & 40 Nodes \\
\hline $\begin{array}{l}\text { Required Wireless } \\
\text { Node Resources }\end{array}$ & Low & Low & High & Low & Low & Low \\
\hline $\begin{array}{l}\text { Requires } \\
\text { Modification to } \\
\text { Bluetooth Access } \\
\text { Point }\end{array}$ & No & No & No & No & Yes & Preferably No \\
\hline $\begin{array}{l}\text { Requires } \\
\text { Modification to } \\
\text { Bluetooth Wireless } \\
\text { Nodes }\end{array}$ & No & $\begin{array}{l}\text { No } \\
\text { (Park Mode } \\
\text { Required) }\end{array}$ & Yes & No & No & If Required \\
\hline Power Usage & Low & Low & $\begin{array}{l}\text { Medium } \\
\text { (M/S nodes) }\end{array}$ & $\begin{array}{l}\text { High } \\
\text { (Routing Nodes) }\end{array}$ & Low & Low \\
\hline $\begin{array}{l}\text { Major Structure } \\
\text { Advantage }\end{array}$ & $\begin{array}{l}\text { Simple } \\
\text { Implementation }\end{array}$ & Single Hop & $\begin{array}{l}\text { Nodes } \\
\text { Always Active }\end{array}$ & $\begin{array}{l}\text { Simple } \\
\text { Implementation }\end{array}$ & $\begin{array}{l}\text { Simple } \\
\text { Implementation }\end{array}$ & \\
\hline $\begin{array}{l}\text { Major Structure } \\
\text { Limitation }\end{array}$ & $\begin{array}{l}\text { Number of } \\
\text { Nodes }\end{array}$ & $\begin{array}{l}\text { Network } \\
\text { Latency }\end{array}$ & $\begin{array}{l}\text { Resource } \\
\text { Intensive }\end{array}$ & $\begin{array}{l}\text { Requires } \\
\text { dedicated sensor } \\
\text { routing nodes }\end{array}$ & $\begin{array}{l}\text { Possible } \\
\text { Modification of } \\
\text { Bluetooth Stack }\end{array}$ & \\
\hline
\end{tabular}

Table 5: Bluetooth Network Structure Summary. 
Table 5 shows that a standard Bluetooth Piconet does not fulfil the node requirements for the designed wireless sensor network for sports monitoring applications. The proposed network structures are required to make the Bluetooth Specification suitable for wireless sensor network purposes.

The Piconet Sharing network structure shares the active member addresses of the Bluetooth Piconet between the connected slaves. The only requirement of this network structure is that the Bluetooth hardware used supports park mode. The Bluetooth Access Point acting as the root node must support functionality to place the devices into park mode and must be able to park up to 30 devices on a single Piconet (as per the Bluetooth Specification). The limiting factor of the Piconet Sharing network structure is the long latencies between active communications on the Piconet. As long as the sensor node is able to buffer the sensor data for the required length of time, this network structure is suitable for wireless sensor networking applications.

The Scatternet Tree network structure forms a tree based network structure by connecting multiple Piconets with bridging nodes to form a Scatternet. This network structure forms a multi-hop network and relies on the bridging nodes to relay the sensor data from the daughter Piconet. If the bridging node loses connection to the Bluetooth Access Point then each device on the Piconet also loses its network connection path. For this reason the Scatternet Tree Structure is best suited to sports monitoring wireless sensor applications were node drop outs are infrequent. The wireless sensor nodes require a large amount of modification to support Scatternet operation. Scatternet operation is resource intensive and will result in a reduced battery life for the bridging nodes.

The Piconet Tree is a similar tree structure to the Scatternet Tree except the bridging nodes are made up of two Bluetooth radios. This reduces the complexity of the software implementation while still allowing an adequate number of sensor nodes to communicate on a Bluetooth network structure. The bridging nodes in this structure only route data back to the access point root node, they cannot support a wireless sensor platform. These non-sensing nodes are the main disadvantage of the Piconet Tree network structure. As this is a tree structure it works best with wireless sensing applications were node drop outs are infrequent. The Piconet Tree network structure is ideal for sports monitoring applications were multiple sensor platforms are attached to an athlete, the sensor platforms relay the data to the bridging node which retransmits the data to the wireless access point route node.

The Multiple Piconet network structure is created by attaching multiple Bluetooth radios to the Bluetooth Access Point. In this manner multiple Piconets can be created and controlled by the root node. This network structure has the potential to give the highest throughput per device of all the proposed network structures. It however requires the Bluetooth Access Point to be modified to support additional Bluetooth radios. 
The Piconet Sharing, Piconet Tree and Multiple Piconet Root Node Structures have been developed in this body of work and represent novel techniques for extending the node limits in Bluetooth networks within the Bluetooth Specification. The Scatternet Tree Bluetooth Structure was developed based upon previously published work [44].

\section{Bluetooth Wireless Sensor Network Simulation}

There are several commercial and open source Bluetooth simulation tools available. Bluehoc is an open source extension for the widely used Network Simulator (NS) open source network simulation software [60]. This software allows the user to create a simple Bluetooth Piconet and measure basic Bluetooth communications statics. An extension to the Bluehoc simulator is called Bluescat that provides limited Scatternet functionality. A commercial product that was considered is called OPNET which provides network simulation functionality [61]. Suitetooth is a simulation module for OPNET that allows communications on standard Bluetooth Piconets to be simulated [62]. Rather than be constrained by the limitations of these simulation tools, a custom program was written in MATLAB [63]. The developed simulation tools were written to compare the theoretical performance of the Bluetooth network structures for wireless sensor network applications. To create the Bluetooth simulations various assumptions were made based upon the Bluetooth specification. The Bluetooth simulations are based upon version 1.1 of the Bluetooth Specification [1].

The purpose of the Bluetooth simulations was to determine the maximum throughput per wireless sensor node for the various Bluetooth Network Structures described in 3 Bluetooth Wireless Sensor Network Design. Those sections of the Bluetooth specification which do not affect network throughput were not implemented in the simulations. Thus Bluetooth frequency hopping, inquiry and paging procedures, radio characteristics and non-Bluetooth interfering noise were not implemented. While frequency hopping was not implemented, the effect of coexisting Piconets was examined by assuming that the Bluetooth frequency hopping sequence is completely random. The Bluetooth network structures are at the proof of concept stage and interference from non-Bluetooth devices has been assumed as negligible. Verification and validation of the developed Bluetooth simulation was completed by producing Bluetooth packet traces from each simulation. These packet traces were compared to the Bluetooth specification to ensure compliance within the assumptions made. Hardware limitations were not considered in these Bluetooth network simulations. Bluetooth hardware limitations are discussed in Chapter 5 Bluetooth Wireless Sensor Network Testing.

The four Bluetooth simulation tools created were; 4.1.1 Standard Piconet Bluetooth Network Structure Simulation, 4.1.2 Bluetooth Piconet Coexistence Simulation, 4.2.1 Piconet Sharing Bluetooth Network Structure Simulation and 4.2.2 Scatternet Tree Bluetooth Network Structure Simulation. Results for the -40 - 
4.2.3 Piconet Tree Bluetooth Network Structure and the 4.2.4 Multiple Piconet Bluetooth Network Structure were calculated from these simulations as they are formed from standard coexisting Bluetooth Piconets. Results from the standard Bluetooth Piconet and Piconet coexistence simulations were used to determine network throughput for these two network structures. Relevant sections of the Bluetooth Specification have been explained for each simulation and compared with the simulated functionality.

Network throughput per node was calculated for each of the Bluetooth network structures under a number of scenarios. Each simulation highlighted various aspects of each structure that needed to be considered in implementation.

\subsection{Standard Bluetooth Piconet Simulations}

A Bluetooth simulation tool was written for standard Piconet communications to verify that the simulation methodology was valid and functioned correctly based upon the Bluetooth Specification. Once the results correlated with those found in the specification, the simulation functionality was expanded. The effect of lost packets due to coexisting Piconets was also examined. Experiments were conducted with Bluetooth packet types to determine the effects of coexisting Piconets on Bluetooth network throughput.

\subsubsection{Standard Piconet Bluetooth Network Structure Simulation}

A communication channel in Bluetooth Piconets is frequency hopped and broken into communication time slots accessed using a time division duplex scheme. Each frequency channel is hopped between at 1600 hops per second and each communication time slot is $625 \mu \mathrm{s}$. Each communication time slot occurs on a different hop frequency (if single slot Bluetooth packets are used on the Piconet). The Piconet master transmits on even numbered time slots and the slaves in the Piconet transmit on the odd number time slots. Slave devices can only transmit on the Piconet once they receive a packet from the master (this packet can either be a data packet or a POLL packet). There are two types of Bluetooth packets Asynchronous Connection-Less (ACL) and Synchronous Connection-Oriented (SCO). SCO packets are used for time bounded data like voice and have the ability to reserve slots on the Piconet to ensure a suitable quality of service. ACL packets are sent asynchronously when there is no SCO traffic. SCO packets were not required in the wireless sensor network and have therefore not been implemented in this simulation. There are three types of ACL packets Data Medium (DMx) rate, Data High (DHx) rate and AUX1 packets. DMx packets contain forward error correction (FEC) and therefore have a smaller maximum payload than DHx packets which have no error correction. DMx packets contain blocks of 10 information bits encoded into a 15bit codeword. This allows the FEC on the receiver to recover single bit errors and detect two bit errors in each codeword. Both DMx and DHx packets contain cyclic redundancy checks to verify data integrity. AUX1 packets contain no error checking or error correction. These 
packets were unsuitable for the intended application and so were not implemented in the simulation. DMx and DHx packets can occupy more than a single Bluetooth time slot. These packets continue to be transmitted on the current frequency hopping channel until the total packet has been sent. The Bluetooth hopping sequence continues hopping, skipping the missed Bluetooth hopping channels. Figure 16 shows an example of channel access on a Bluetooth Piconet with two active slaves and Table 6 is a summary of the implemented ACL packet types.

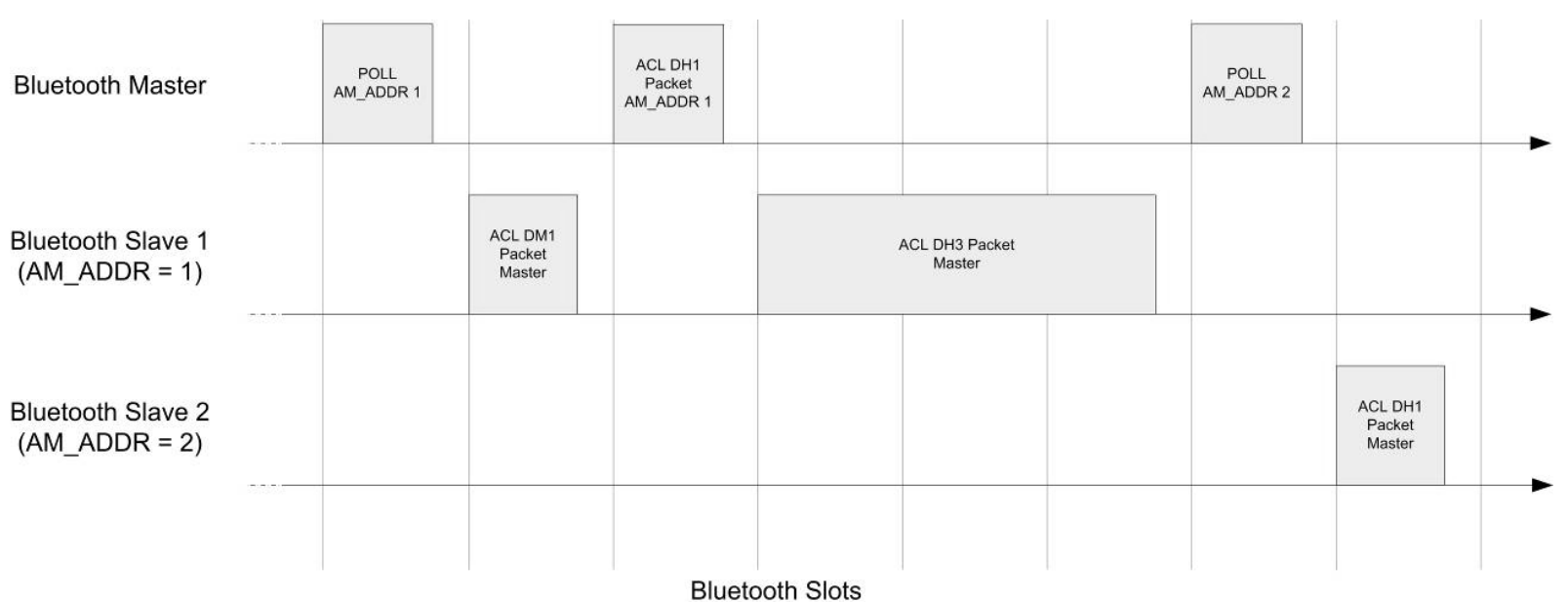

Figure 16: Example of Channel Access on a Bluetooth Piconet with Two Active Slaves

\begin{tabular}{|c|c|c|c|c|c|c|}
\hline $\begin{array}{l}\text { Bluetooth } \\
\text { Packet } \\
\text { Type }\end{array}$ & $\begin{array}{l}\text { Number } \\
\text { of Time } \\
\text { Slots }\end{array}$ & $\begin{array}{l}\text { Payload } \\
\text { Header } \\
\text { (Bytes) }\end{array}$ & $\begin{array}{l}\text { User } \\
\text { Payload } \\
\text { (Bytes) }\end{array}$ & $\begin{array}{l}\text { Forward } \\
\text { Error } \\
\text { Correction } \\
\text { (FEC) }\end{array}$ & $\begin{array}{l}\text { Cyclic } \\
\text { Redundancy } \\
\text { Check } \\
\text { (CRC) }\end{array}$ & $\begin{array}{l}\text { Asymmetric } \\
\text { Maximum } \\
\text { Data Rate } \\
\text { (kbps) }\end{array}$ \\
\hline$\overline{\mathrm{DM} 1}$ & 1 & 1 & $0-17$ & $2 / 3$ & Yes & 108.8 \\
\hline$\overline{\mathrm{DH} 1}$ & 1 & 1 & $0-27$ & No & Yes & 172.8 \\
\hline DM3 & 3 & 2 & $0-121$ & $2 / 3$ & Yes & 387.2 \\
\hline $\mathrm{DH} 3$ & 3 & 2 & $0-183$ & No & Yes & 585.6 \\
\hline DM5 & 5 & 2 & $0-224$ & $2 / 3$ & Yes & 477.8 \\
\hline DH5 & 5 & 2 & $0-339$ & No & Yes & 723.2 \\
\hline
\end{tabular}

Table 6: Bluetooth ACL Packet Types [1]

The following limitations and assumptions were used in the development of this Bluetooth Piconet simulation:

- The Bluetooth communication link is never lost.

This simulation was designed to calculate the maximum theoretical throughput of the Bluetooth

Piconet. Inquiry, Paging and connection loss was not included. 
- There is no noise or interference.

The simulation assumes a perfect environment where path loss and interfering devices do not exist. Directional antennas are being used on each of the root nodes which should help to minimise the effect of interfering devices.

- There is no frequency hopping.

As this was designed for a perfect environment frequency hopping has no effect on network throughput and has therefore not been included.

- Data is only generated by slaves on the Piconet.

In the desired application, the master will only be receiving data from the wireless sensor nodes acting as slave devices. For this reason the master never transmits data packets to the slaves, only POLL packets are transmitted to each of the connected slaves.

Figure 17 and Figure 18 are the flow charts of the Standard Bluetooth Piconet Network Structure Simulation. Simulation parameters such as simulation length, the number of slaves in the Piconet and the ACL packet type for each slave are all configurable. These simulation parameters are summarised in Table 7. Appendix A contains the full source code of the developed simulation software for Bluetooth Piconet communications for different packet types. Figure 19 is a sample trace for a standard Bluetooth Piconet with seven slaves for a variety of Bluetooth packet types. Slaves 1 and 7 are transmitting DM1 packets, Slave 2 is transmitting DH1 packets, Slave 3 is transmitting DM3 packets, Slave 4 is transmitting DH3 packets, Slave 5 is transmitting DM5 packets and Slave 6 is transmitting DH5 packets. 


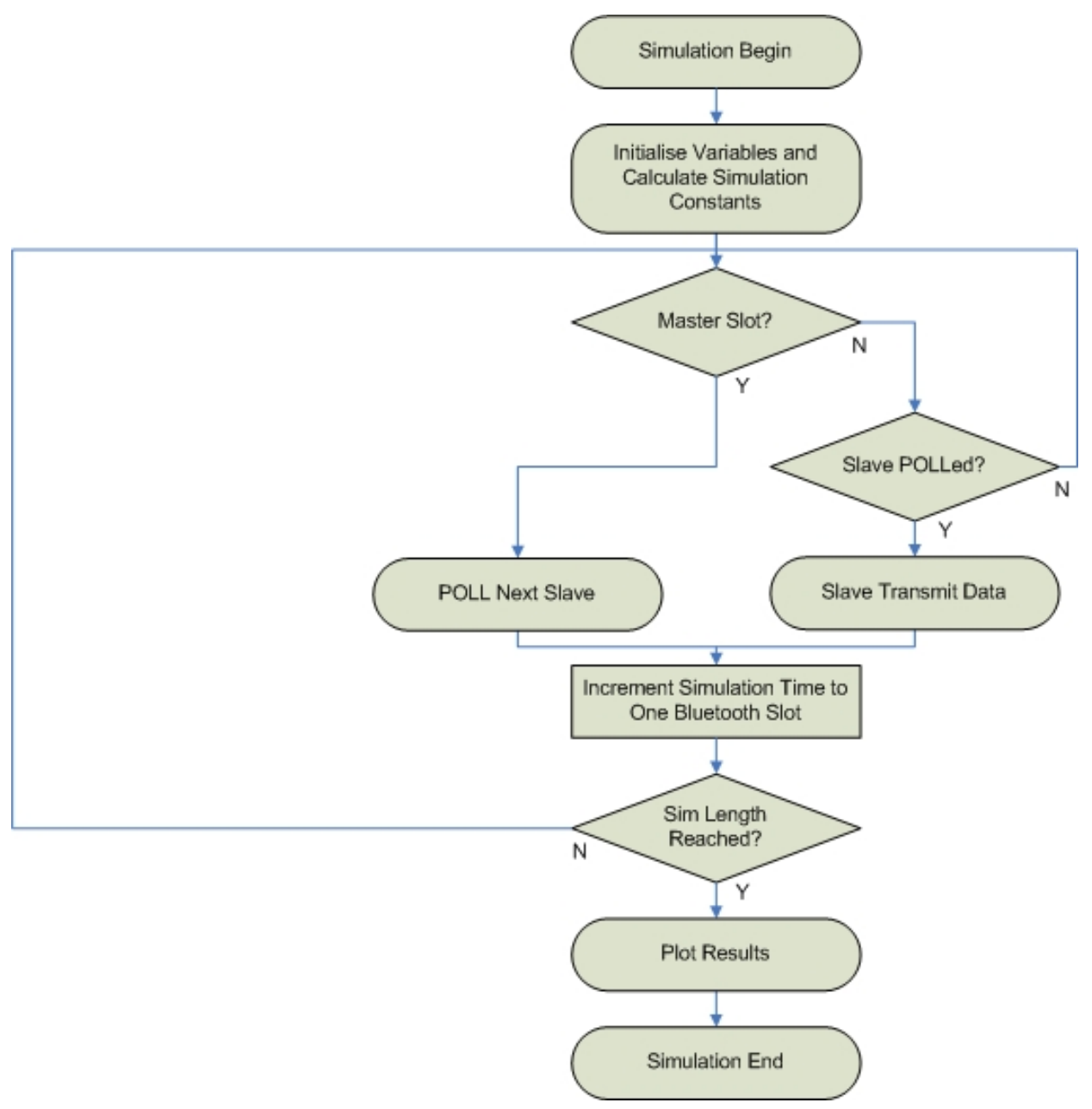

Figure 17: Standard Bluetooth Piconet Network Structure Simulation Main Flow Diagram 

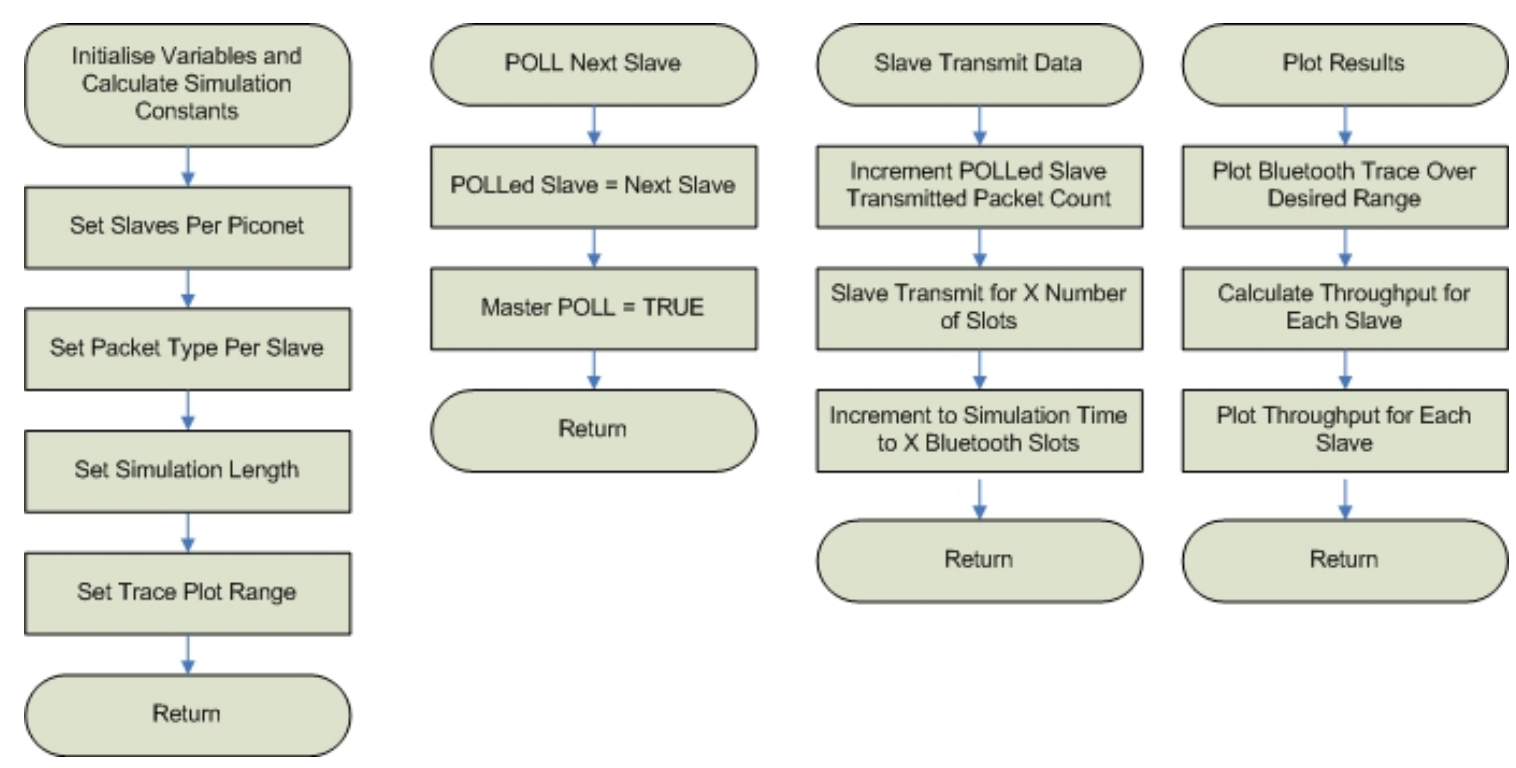

Figure 18: Standard Bluetooth Piconet Network Structure Simulation Flow Diagram

\begin{tabular}{|l|l|l|}
\hline Simulation Parameter & \multicolumn{1}{|l|}{ Description (units) } & Default Value \\
\hline no_slots & Length of Simulation (slots) & 48000 \\
\hline no_slaves & $\begin{array}{l}\text { Number of Slaves per Piconet } \\
\text { (devices) }\end{array}$ & 7 \\
\hline slave_packet_type(X) & $\begin{array}{l}\text { ACL Packet Type Transmitted } \\
\text { by Slave X (ACL Packet Type) }\end{array}$ & $\begin{array}{l}\text { slave_packet_type(1)= DM1 } \\
\text { slave_packet_type(2)= DH1 } \\
\text { slave_packet_type(3)= DM3 } \\
\text { slave_packet_type(4)= DH3 } \\
\text { slave_packet_type(5)= DM5 } \\
\text { slave_packet_type(6)= DH5 } \\
\text { slave_packet_type(7)= DM1 }\end{array}$ \\
\hline bt_trace_min & $\begin{array}{l}\text { Minimum Time Value for the } \\
\text { Bluetooth Trace Output (slots) }\end{array}$ & 1 \\
\hline bt_trace_max & $\begin{array}{l}\text { Maximum Time Value for the } \\
\text { Bluetooth Trace Output (slots) }\end{array}$ & 80 \\
\hline
\end{tabular}

Table 7: Standard Bluetooth Piconet Network Structure Simulation Parameters 


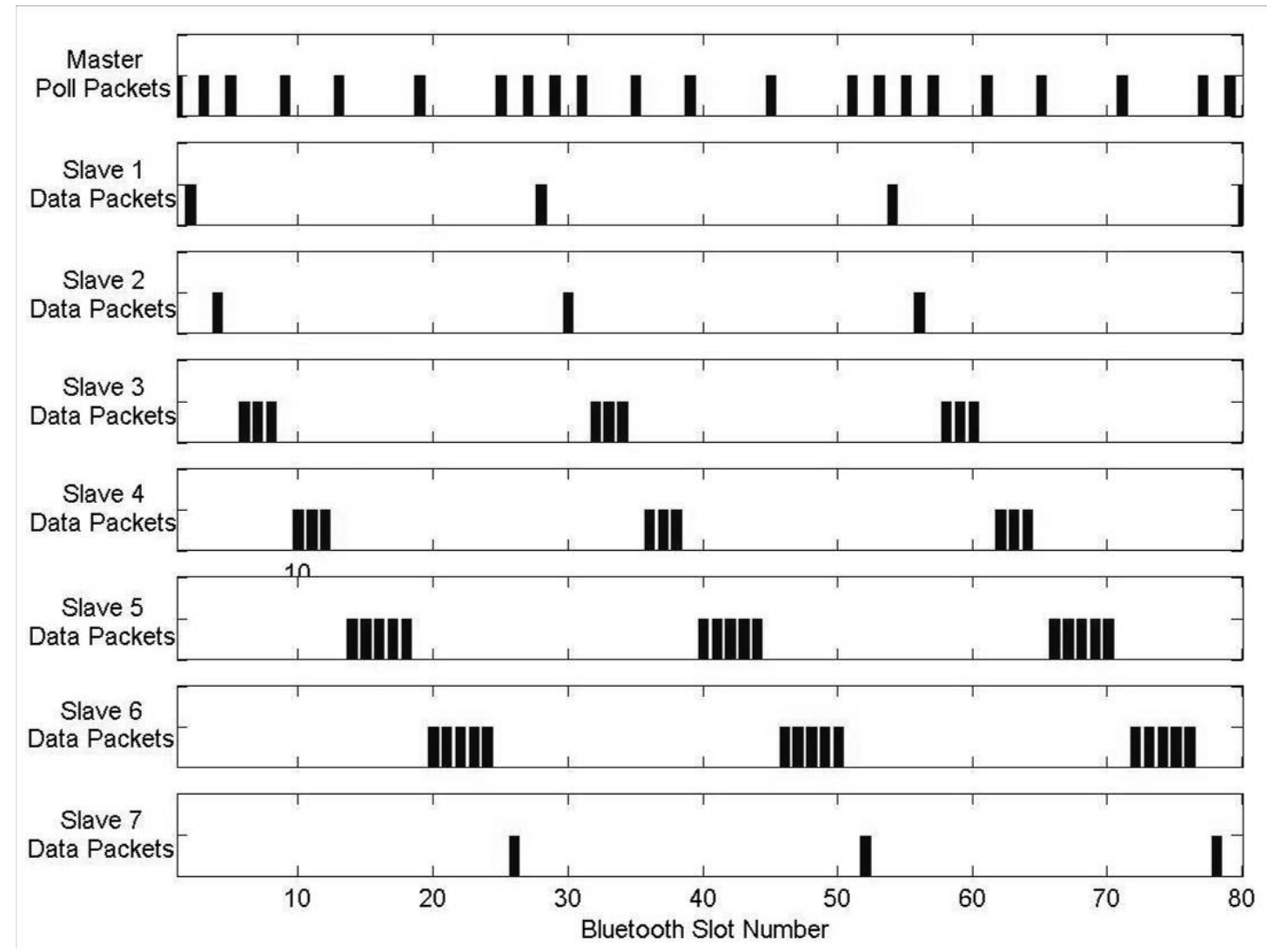

Figure 19: Sample Output for Standard Bluetooth Piconet Simulation

Figure 19 shows the Piconet master repeatedly sending POLL packets to each of the slaves, with each slave responding with a data packet. Time on the Bluetooth Piconet is broken up into Bluetooth time slots of 625 micro-seconds. In this example slave 1 and 2 were set to transmit packets that are one slot in length; Slave 1 is using DM1 packets and Slave 2 us using DH1 packets. This means that in this noise free simulation environment Slave 2 will have a higher throughput. This may not be the case in an environment containing noise as $\mathrm{DHx}$ packets do not contain any error correction capabilities. With this simulation using different packet types per slave, it is expected that each slave will have a different network throughput. This is evident in Figure 20 which shows the throughput for each slave for the Bluetooth simulation trace that appears in Figure 19. 


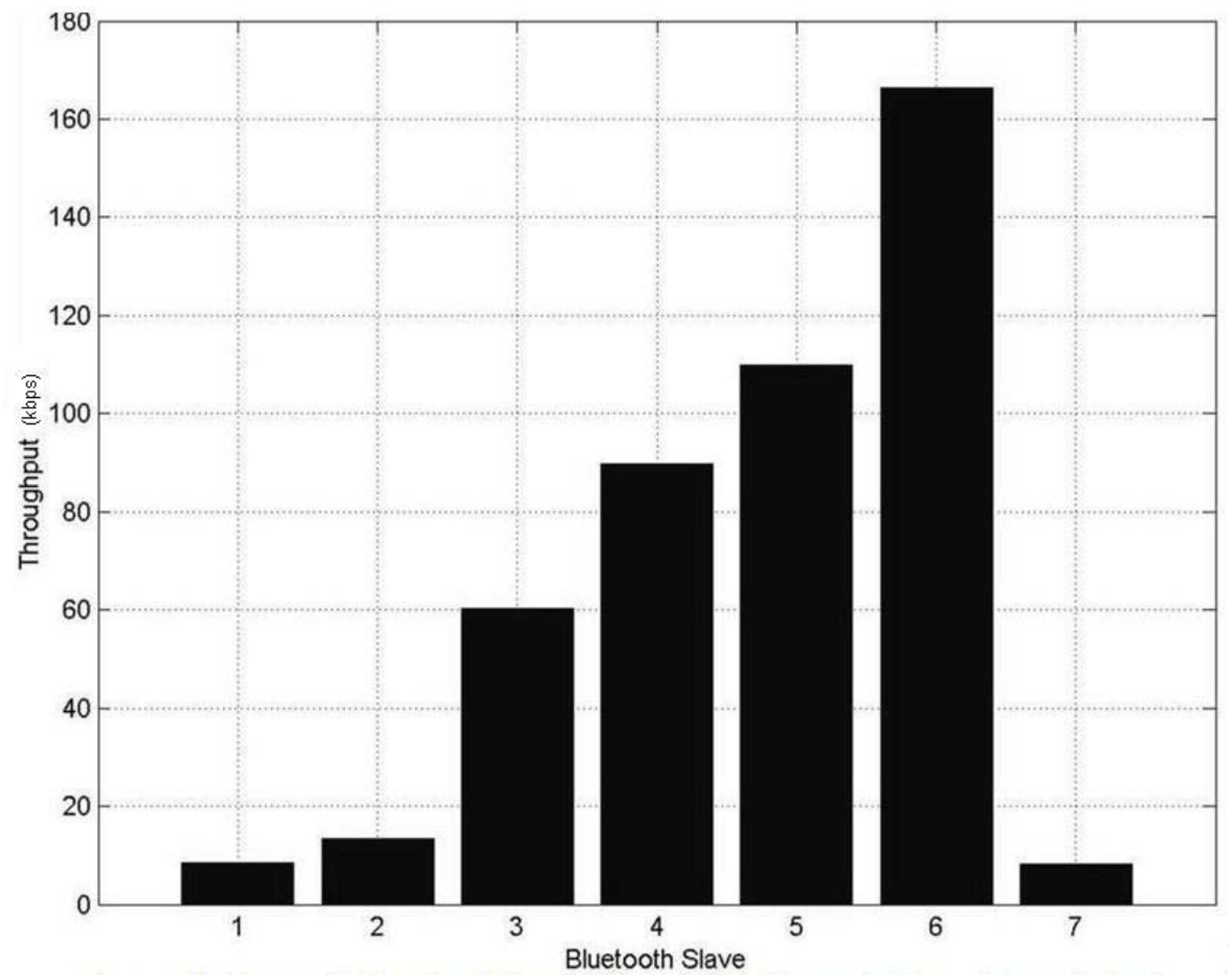

Figure 20: Example Standard Piconet Simulation Throughput per Slave Output

The simulation program was run with a single slave to determine the maximum asymmetric data rate of a Bluetooth Piconet for each of the ACL packet types. These results are compared with the values from the Bluetooth Specification in Table 8.

\begin{tabular}{|lll|}
$\begin{array}{l}\text { Bluetooth } \\
\text { Packet Type }\end{array}$ & $\begin{array}{l}\text { Simulated Throughput } \\
\text { for Single Slave } \\
\text { (kbps) }\end{array}$ & $\begin{array}{l}\text { Bluetooth Specification } \\
\text { Asymmetric Maximum } \\
\text { Data Rate (kbps) [1] }\end{array}$ \\
\hline DM1 & 108.8000 & 108.8 \\
\hline DH1 & 172.8000 & 172.8 \\
\hline DM3 & 387.2000 & 387.2 \\
\hline DH3 & 585.6000 & 585.6 \\
\hline DM5 & 477.8667 & 477.8 \\
\hline DH5 & 723.2000 & 723.2 \\
\hline
\end{tabular}


The simulated throughput calculated over 5 minutes (480000 slots) was the same as that listed in the Bluetooth Specification (except for the rounding used in the specification for DM5 packets) [1]. The complete throughput results for Standard Bluetooth Piconets using different ACL packets are given in Figure 21.

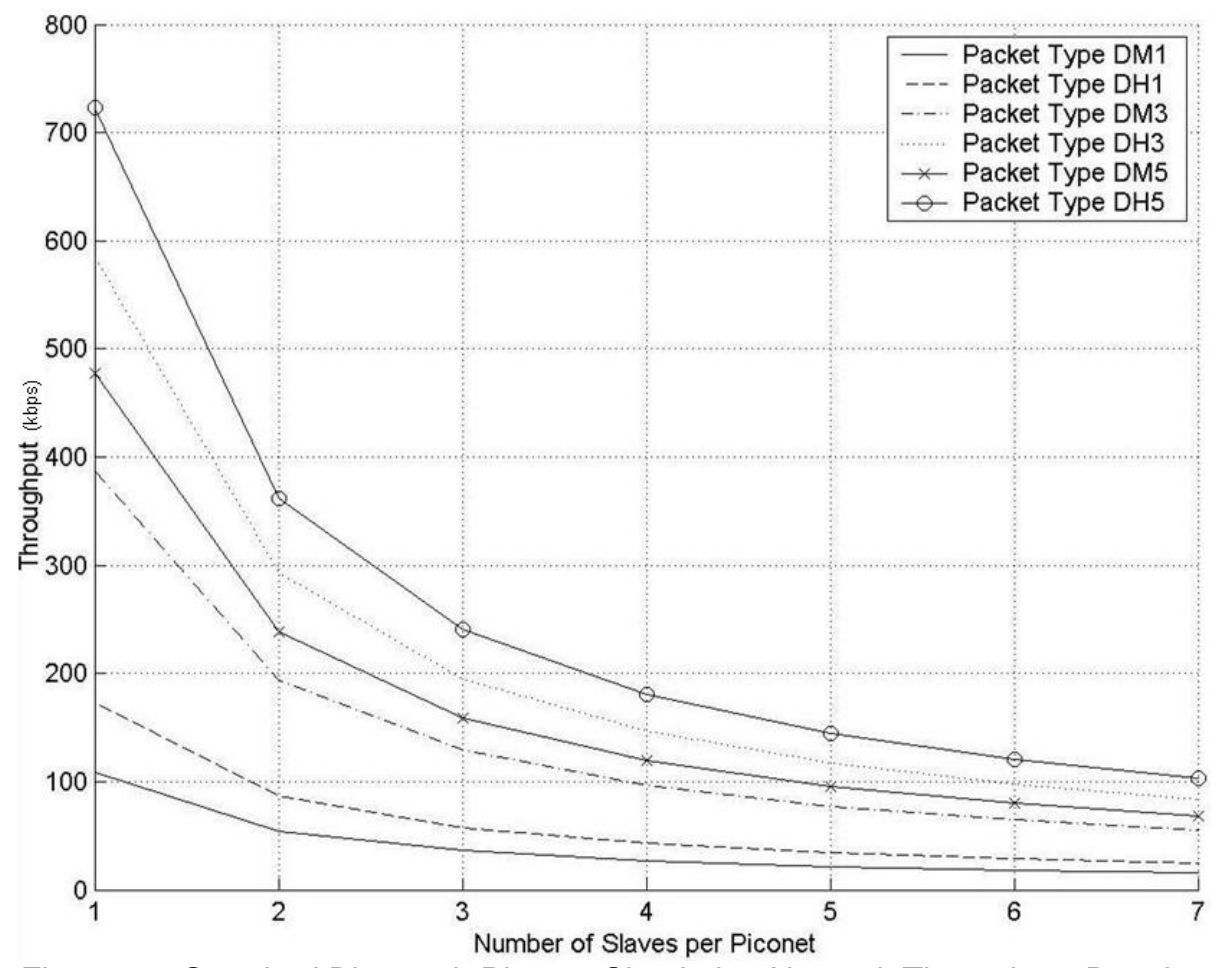

Figure 21: Standard Bluetooth Piconet Simulation Network Throughput Results

As expected, the larger payloads result in higher data throughput in a perfect noise free simulation environment. This basic test verified the accuracy of the simulation. The next step was to add interference from adjacent coexisting Piconets.

\subsubsection{Bluetooth Piconet Coexistence Simulation}

The Bluetooth communications channel frequency hops over 79 hop frequencies to avoid interference from other radio devices in the $2.4 \mathrm{GHz}$ frequency range (including other Bluetooth Piconets). The hopping sequence for each Piconet is defined by the master device's Bluetooth Address and the current phase offset of the hopping pattern is determined by the master device's clock. If there are multiple Piconets coexisting in the same physical location, the hoping sequences will occasionally fall on the same hop channel and then interfere with each other. This means that the larger the number of coexisting Bluetooth Piconets, the more collisions that will occur between hop frequencies and the lower the overall throughput of the Piconet will fall. Bluetooth has three forms of error correction: 
- A Three times repetition of the packet header

- A shortened hamming code for DMx payload data

- An automatic repeat request scheme.

The Standard Bluetooth Piconet Simulation program was modified to include interference from coexisting Piconets, assuming that if interference occurs that error correction is not possible and the packet must be retransmitted.

Many of the Bluetooth structures contain multiple Piconets that will cause interference to each other. By assuming that the hopping sequence randomly hops between the 79 hop channels the probability that a packet will be lost by interference from a coexisting Piconet can be described by Equation 1 .

$$
\begin{aligned}
& P_{\text {error }}=1-\left(1-\frac{1}{79}\right)^{(n-1)} \text { Equation } 1 \\
& \text { Where: } \\
& \begin{array}{ll}
P_{\text {error }} & =\text { Probability that a packet will be lost } \\
n & =\text { Number of coexisting Piconets }
\end{array}
\end{aligned}
$$

Coexisting Piconets sending single slot packets have the greatest potential to cause interference to adjacent Piconets as every communications time slot is on a different Bluetooth hop frequency. This equation assumes that the coexisting Piconets send single slot packets only (resulting in the maximum packet loss). By incorporating Equation 1 into the Bluetooth simulation the effect of Bluetooth Piconet coexistence was examined. The following simulation limitations and assumptions were made in this Bluetooth Piconet coexistence simulation:

- The Bluetooth communication link is never lost.

This simulation was designed to measure the impact of Piconet coexistence on the maximum theoretical throughput of the Bluetooth Piconet. Inquiry, Paging and connection loss were not included.

- There is no noise or interference.

The simulation assumes a perfect environment where the path loss is zero and there are no interfering non-Bluetooth devices. Directional antennas were used on each of the root nodes to eliminate interfering devices.

- The Bluetooth frequency hopping sequence was approximated as a random sequence.

This eliminated the need to take into account the complex frequency hop algorithms which rely on the Bluetooth master's BD_ADDR and current on board clock value. This approximation had only 
a minor effect on the simulation results as each coexisting Piconet frequency hopping sequence appears seemingly random without knowledge of the Bluetooth master's parameters.

- Data was only generated by slaves on the Piconet.

The master only transmited POLL packets to each of the connected slaves. When a Master POLL packet was lost due to coexisting Piconet interference, the corresponding slave lost an opportunity to transmit. The master does not resend lost POLL packets.

- When a frequency hopping channel collision occurred, the entire Bluetooth packet is lost.

It was assumed that error correction was not possible in DMx packets because interference from coexisting Piconets caused more than a single bit error in each FEC codeword. This meant that DMx packets suffering from Piconet coexistence interference were not recoverable using FEC and must be retransmitted.

The flow diagrams for the implemented Bluetooth Piconet Coexistence Simulation are given in Figure 22 and Figure 23. The developed code for the Bluetooth Piconet Coexistence Simulation can be found in Appendix B. The simulation parameters for the Bluetooth Coexistence Simulation are summarised in Table 9. 


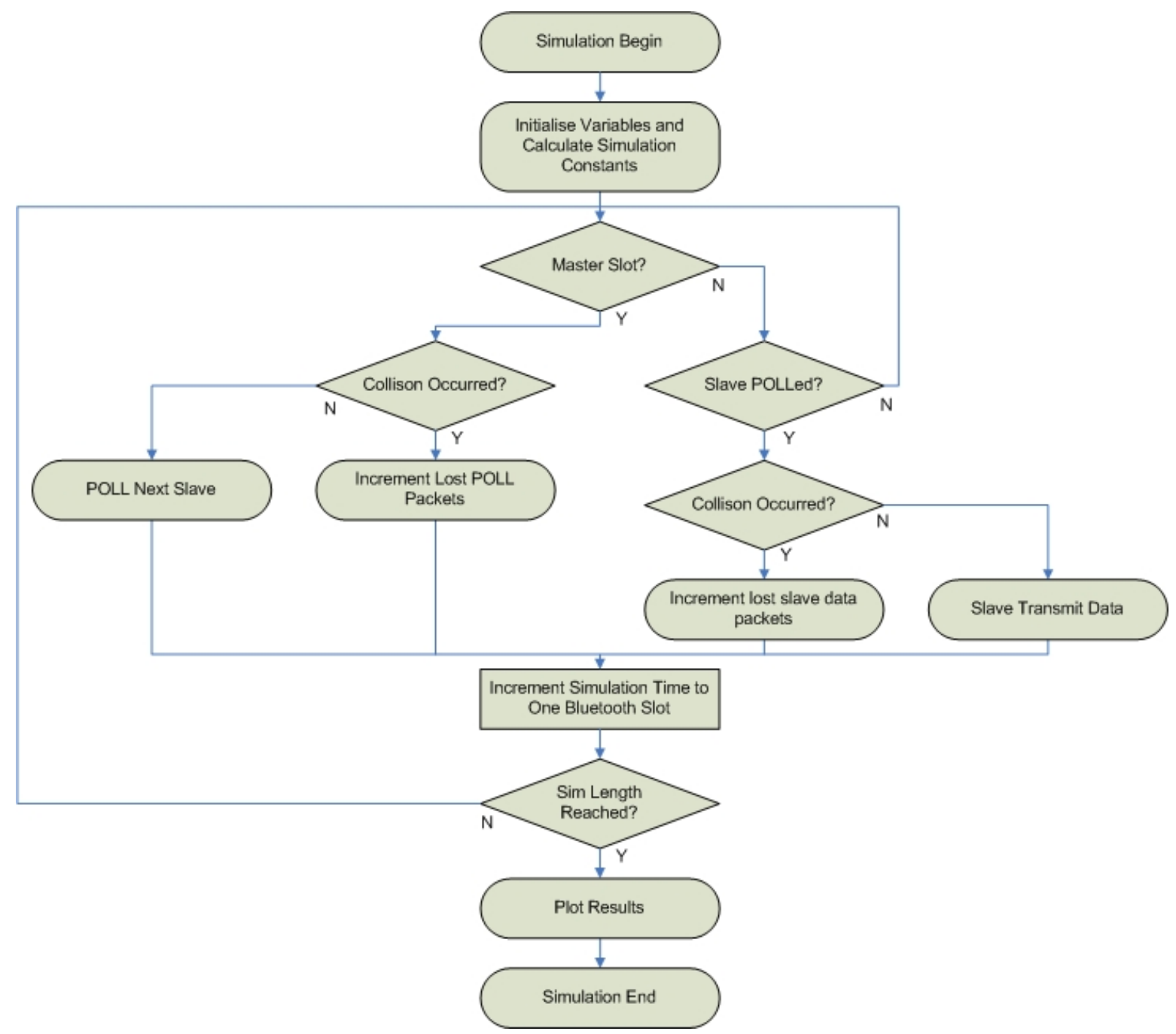

Figure 22: Bluetooth Piconet Coexistence Simulation Main Flow Diagram 

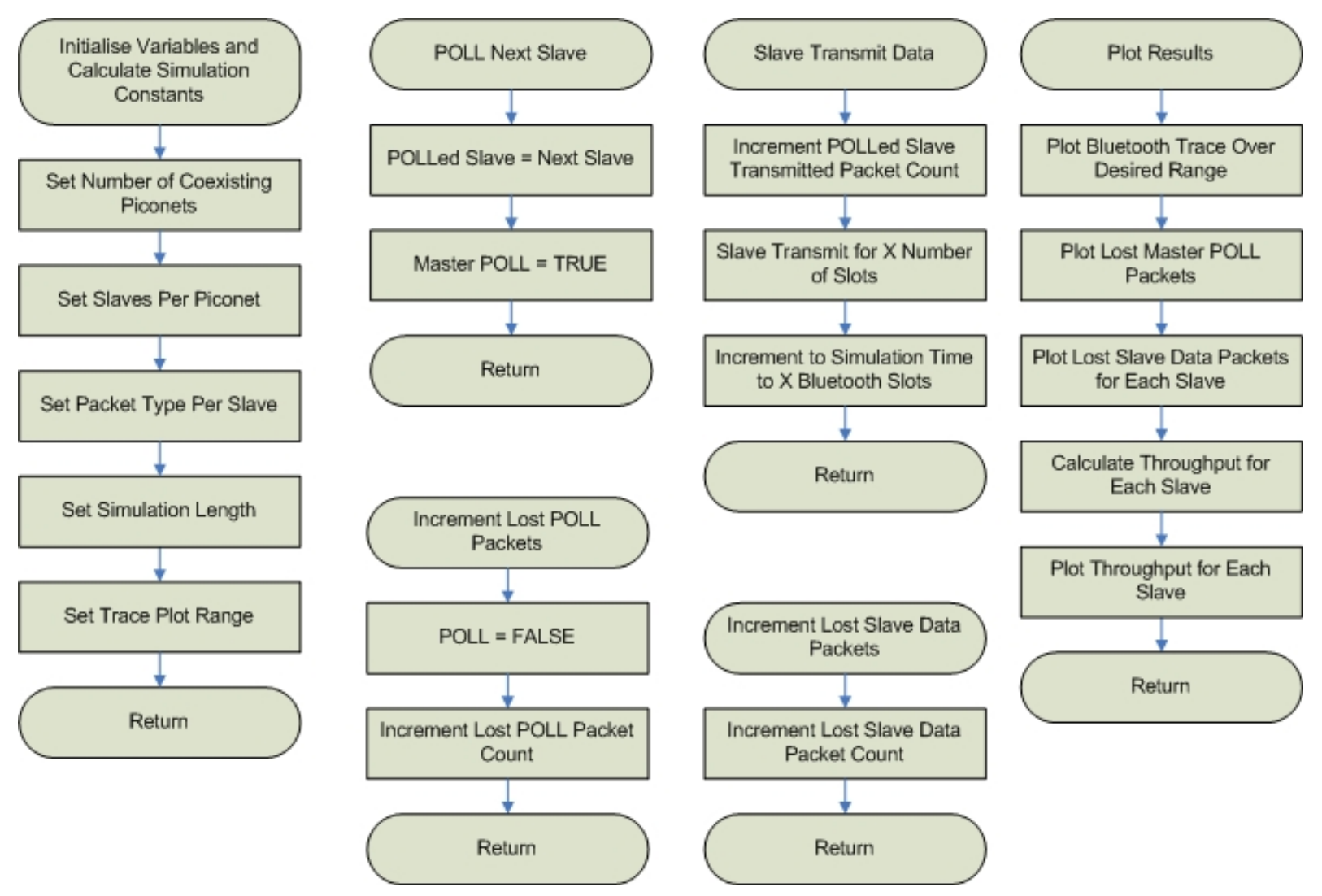

Figure 23: Bluetooth Piconet Coexistence Simulation Flow Diagram

\begin{tabular}{|l|l|l|}
\hline \multicolumn{1}{|l|}{ Simulation Parameter } & Description (units) & Default Value \\
\hline no_slots & Length of Simulation (slots) & 48000 \\
\hline no_slaves & $\begin{array}{l}\text { Number of Slaves per Piconet } \\
\text { (devices) }\end{array}$ & 4 \\
\hline slave_packet_type(X) & $\begin{array}{l}\text { ACL Packet Type Transmitted } \\
\text { by Slave X (ACL Packet Type) }\end{array}$ & $\begin{array}{l}\text { slave_packet_type(1)= DH5 } \\
\text { slave_packet_type(2)= DH5 } \\
\text { slave_packet_type(3)= DH5 } \\
\text { slave_packet_type(4)= DH5 }\end{array}$ \\
\hline no_piconets & $\begin{array}{l}\text { Number of Coexisting Piconets } \\
\text { (Piconets) }\end{array}$ & 4 \\
\hline bt_trace_min & $\begin{array}{l}\text { Minimum Time Value for the } \\
\text { Bluetooth Trace Output (slots) }\end{array}$ & 1 \\
\hline bt_trace_max & $\begin{array}{l}\text { Maximum Time Value for the } \\
\text { Bluetooth Trace Output (slots) }\end{array}$ & 80 \\
\hline
\end{tabular}

Table 9: Bluetooth Piconet Coexistence Simulation Parameters 
Figure 24 is a sample Bluetooth packet trace for a Piconet with four slaves. Slave 1 and 2 were defined as sending DH3 packets and Slave 3 and 4 sending DH5 packets. The grey colour slots indicate that a collision has occurred with a coexisting Piconet and the packet is lost. In this example there were 10 coexisting Piconets sending single slot packets.
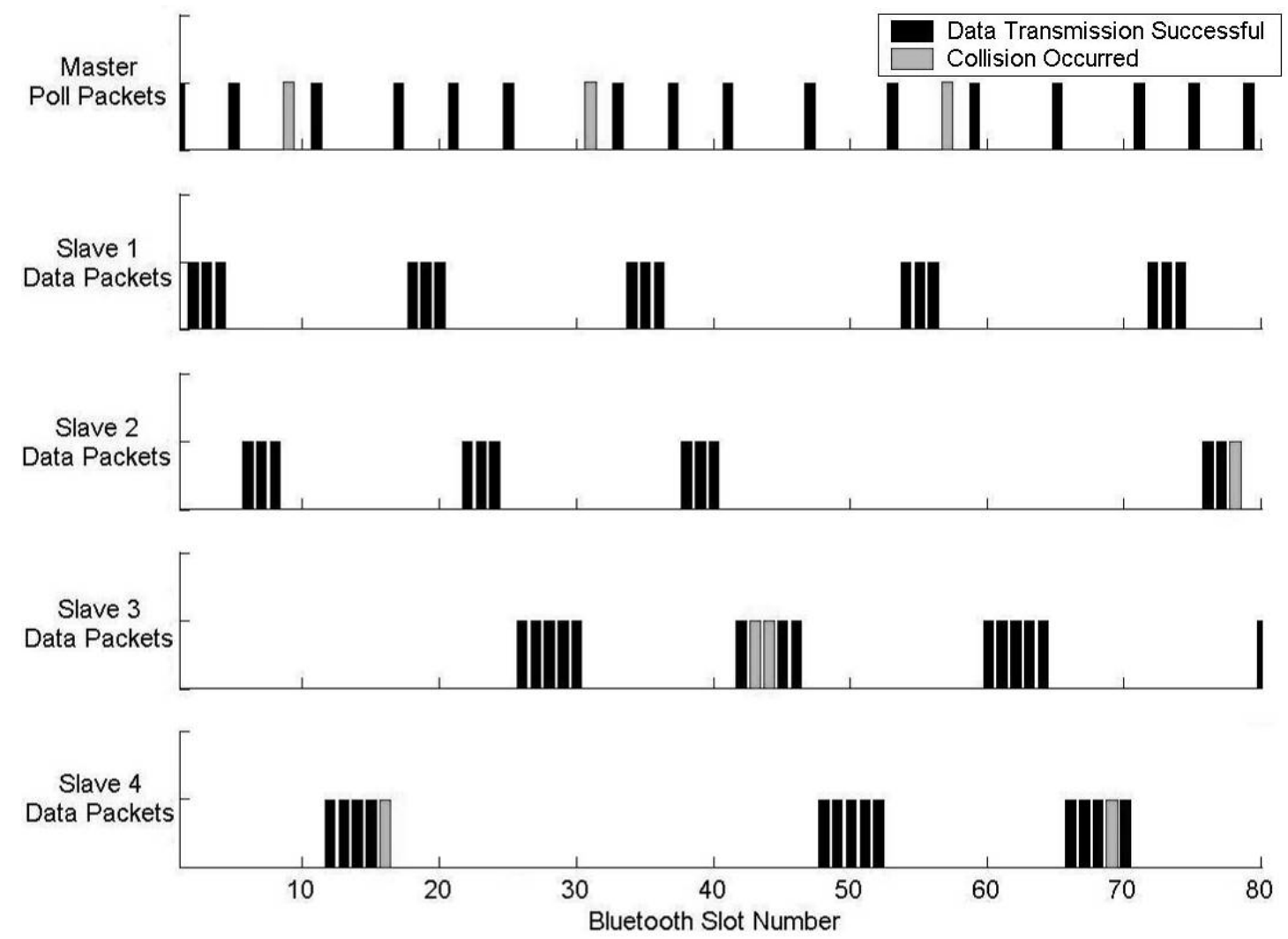

Figure 24: Sample Piconet Coexistence Bluetooth Trace Output

Figure 24 above shows POLL packets from the master being lost due to Piconet interference. Note that at slot number 9 the corresponding slave also loses the opportunity to communicate on the Piconet. Figure 25 shows the corresponding throughput per slave output for the above Piconet coexistence example simulation. 


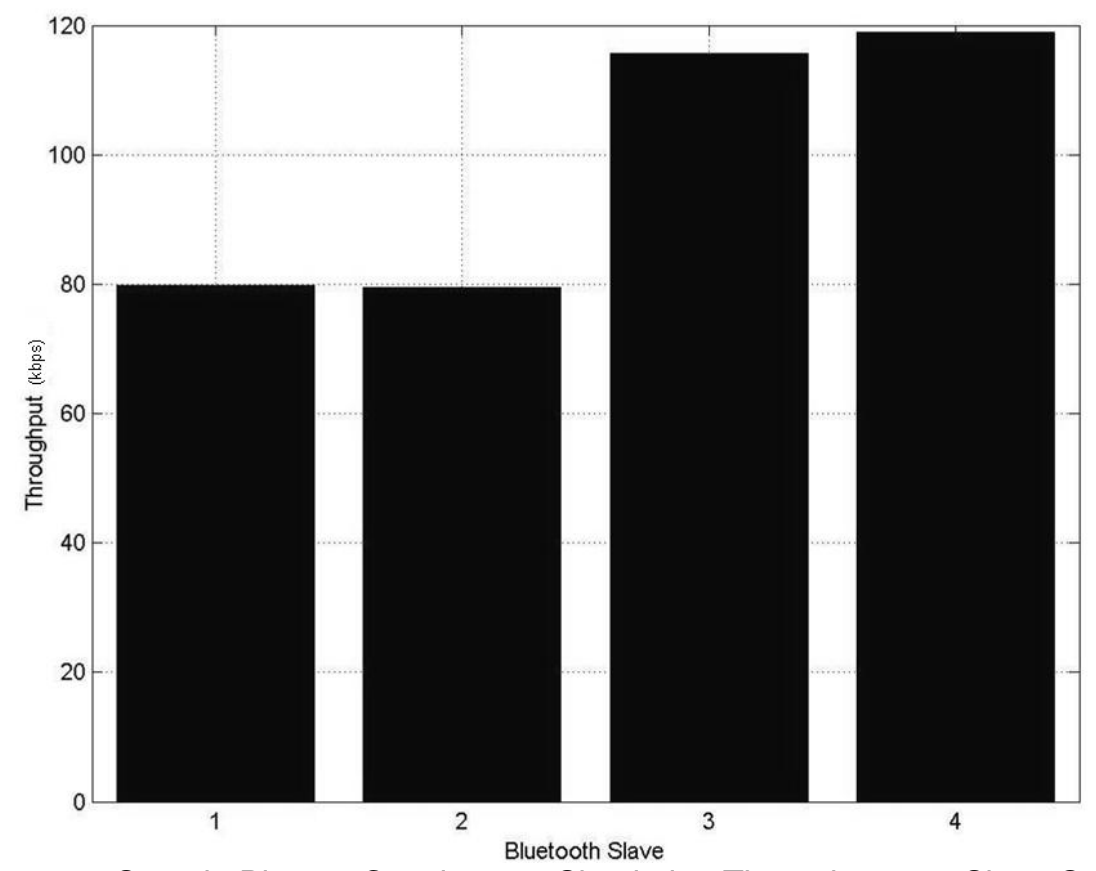

Figure 25: Sample Piconet Coexistence Simulation Throughput per Slave Output

As coexisting Bluetooth Piconets have a pseudo random (assumed random in the simulation) hopping sequence the throughput for each of the slaves was expected to be affected by a different amount. It can be seen that as the number of coexisting Piconets increases, the network throughput for each slave decreases. Figure 26 shows this for each of the Bluetooth packet types for a point to point Bluetooth link. 


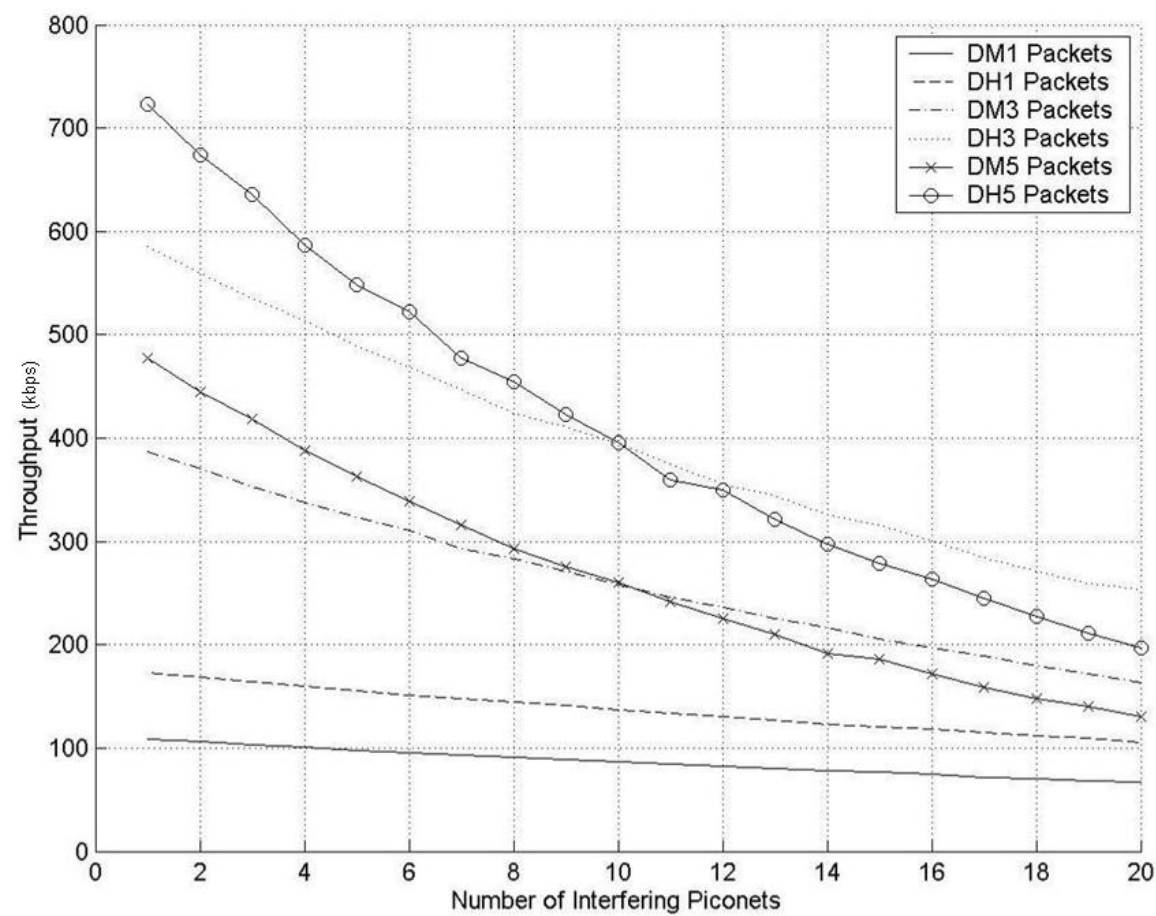

Figure 26: Effect of Coexisting Piconets on Network Throughput.

If one slot of the packet is corrupted the whole packet must be resent, meaning that the larger packets are more likely to require retransmission. Figure 26 demonstrates the decrease in throughput for a point to point Bluetooth link as the number of coexisting Piconets increases. It can be seen that for ten or more coexisting Piconets, a greater throughput can be achieved on the Piconet by transmitting smaller packet types with a reduced payload size. This is because in an environment with a large number of coexisting Piconets smaller packets have a higher probability of being received.

\subsection{Bluetooth Network Structure Simulations}

Bluetooth structure simulation programs were written to compare the theoretical performance of each proposed Bluetooth network structure described in 3 Bluetooth Wireless Sensor Network Design. Extending the Standard Bluetooth Piconet Structure Simulation (outlined in 4.1.1) allowed each of the Bluetooth network structures to be compared and evaluated against theoretical throughput values. Sections of the Bluetooth Specification relevant to each of the network structures were explained and compared with the simulated outputs. Each simulation was designed to provide valuable insight into the operational characteristics of each structure. Results highlighted some important considerations when implementing each structure in hardware. 


\subsubsection{Piconet Sharing Bluetooth Network Structure Simulation}

The Bluetooth specification limits the number of active device to eight per Piconet, one master and seven active slaves. Additional slaves can be connected to a Bluetooth Piconet if they are placed in the low power park mode, where the device gives up the assigned Bluetooth active member address (AM_ADDR) and receives a parked member address (PM_ADDR) and an access request address (AR_ADDR). A full description of the Bluetooth parking method can be found in [1] and is only summarised in this section.

The PM_ADDR and AR_ADDR are 8 bit numbers allowing up to 255 devices to be in park mode. The devices unique Bluetooth Address (BD_ADDR) can also be used when parking and unparking the device. If this method of parking is used there is no limit to the number of parked devices on a Bluetooth Piconet. In practice this is not the case due to embedded devices having strict memory limitations (this is discussed further in 5 Bluetooth Wireless Sensor Network Testing). Each device is assigned a unique PM_ADDR (which is used in master initiated unparking sequences) and device can share AR_ADDR (which are used in slave initiated unparking sequences). Devices that are not assigned PM_ADDR (have all zero PM_ADDR) are unparked using the devices BD_ADDR [1]. The Bluetooth specification uses the Link Manager Protocol stack layer to control Bluetooth parking and unparking of devices. These commands are sent through link manager protocol (LMP) packets.

A Piconet master parks an active slave by sending a LMP park request packet to the slave. Once the slave has received the request to be parked it immediately responds with a LMP accepted packet and starts a timer for six times $T_{\text {poll }}$. Where $T_{\text {poll }}$ is the maximum time allowed between transmissions from the Piconet master to a particular slave on the Piconet. Once $T_{\text {poll }}$ has passed or the slave receives an acknowledgement from the Baseband layer that slave is in park mode. When the Master receives the slaves LMP accepted packet it must wait six times $T_{\text {poll }}$ before using the parked slaves AM_ADDR.

Parked slaves remain synchronised to the Piconet by listening to the beacon channel that is established when a slave is parked. The slave receives the beacon channel parameters when it is placed into park mode. As well as synchronisation, the park beacon exists to allow messages to be passed between the parked slaves and the Piconet master. It is in the park beacon that parked devices are unparked. Figure 27 demonstrates the structure of the Bluetooth beacon channel utilised when devices are in park mode. 


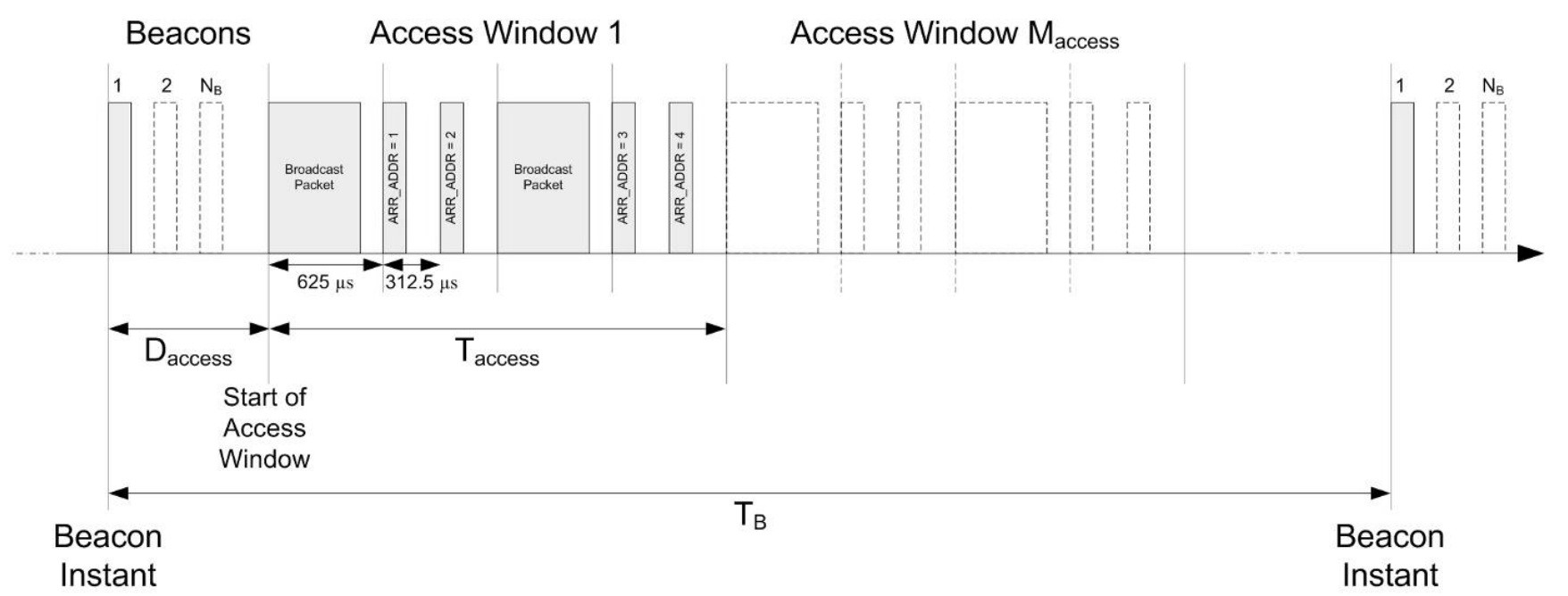

Figure 27: Bluetooth Park Mode Beacon Channel [1]

The number of beacon slots $\left(N_{B}\right)$ and number of slots between beacons $\left(T_{B}\right)$ are chosen based on the Piconet environment. The values are chosen to ensure that there is sufficient beacon slots available so that a parked slave can successfully synchronise in the presence of noise. These beacon slots are used to transmit LMP unpark packets to the slaves. As the packets transmitted in the beacon slots are broadcast packets, any of the parked slaves can use these packets to synchronise with the Piconet. If there are no awaiting master slave transmissions, the master transmits NULL packets so that the slaves may still synchronise with the Piconet. There is a fixed period between the beacon instance and the start of the access window ( $\left.D_{\text {access }}\right)$. The beacon access window is where parked slaves may issue requests to be unparked by the master of the Piconet. The width of the Access Window ( $\left.T_{\text {access }}\right)$ is set based upon the number of AR_ADDR that have been issued to the parked slaves on the Piconet. To increase the reliability of the access window it may be repeated more than once (defined by $\mathrm{M}_{\text {access}}$ ). Bluetooth version 1.1 only specifies the polling technique for channel access in the beacon access window. The access window is a time division duplex scheme similar to that used for channel access in normal Piconet communications. A parked slave can only send a LMP packet to request to be unparked if the previous broadcast packet received from the master was a POLL packet for the slaves AR_ADDR. Slaves with one of the two matching AR_ADDRs can then request to be unparked in the corresponding ID packet half slots. When a slave receives a LMP unpark request it becomes active on the Piconet using the AM_ADDR supplied by the LMP packet. It then waits for a POLL packet from the master, to which it responds with a LMP accepted packet. The slave is now an active member of the Bluetooth Piconet again. 
The following simulation limitations and assumptions were made in this Bluetooth Piconet Sharing Simulation:

- The Bluetooth communication link was never lost.

This simulation was designed to measure the maximum theoretical throughput of the Bluetooth Piconet Sharing Structure. Inquiry, Paging and connection loss was not included in the simulation.

- There was no noise or interference.

The simulation assumes a perfect environment where path loss is zero and there are no interfering non-Bluetooth devices. Directional antennas were used on each of the root nodes, which help to eliminate the effect of interfering (non-Bluetooth) devices. For this reason the park beacon access window was only transmitted once $\left(M_{\text {access }}=1\right)$.

- Data was only generated by slaves on the Piconet.

The master only transmits POLL packets to each of the connected slaves. In the desired application only the mobile nodes generate wireless sensor data.

- $\mathrm{T}_{\text {poll }}$ was set to 50 slots.

A typical value for $T_{\text {poll }}$ was obtained from a Bluetooth Piconet implemented in hardware. The Piconet was sending DM5 / DH5 packets and returned a $T_{\text {poll }}$ value of 43 .

- Slaves were parked / unparked using PM_ADDR and not their BD_ADDR.

This means that up to seven slaves were unparked using a single LMP unpark request packet. If the BD_ADDR was used, only two devices can be unparked at a time and more LMP unpark request packets would be required. This results in a small reduction in overall network throughput.

- $\quad$ Seven AR_ADDR were issued to parked slaves.

It has been assumed that there was a maximum of seven AR_ADDR issued to the parked slaves on the Piconet. If more than seven AR_ADDR are issued, the parking beacons access window increases in size $\left(T_{\text {access }}\right)$. This results in a small reduction in overall network throughput.

- Beacons occupied a maximum of 2 slots.

The number of beacon slots $\left(N_{B}\right)$ was limited so that delay from beacon instant to access window ( $D_{\text {access }}$ ) was 2 slots. A larger value of $N_{B}$ would increase the length of time between the beacon instant and the access window. This would result in a small reduction in overall network throughput. 
- Number of slots between beacon instants $\left(T_{B}\right)$ was 2048 .

A typical value for $T_{B}$ was obtained from a Bluetooth Piconet implemented in hardware. The returned park beacon interval was 2048 slots (1.28 seconds). In standard Bluetooth hardware implementations $T_{B}$ can be varied when parking devices and this is also the case in this simulation.

The flow charts for the implemented Piconet Sharing Structure Simulation appear as Figure 28 and Figure 29. The implement software for the Piconet Sharing Structure Simulation has been included as Appendix C. 


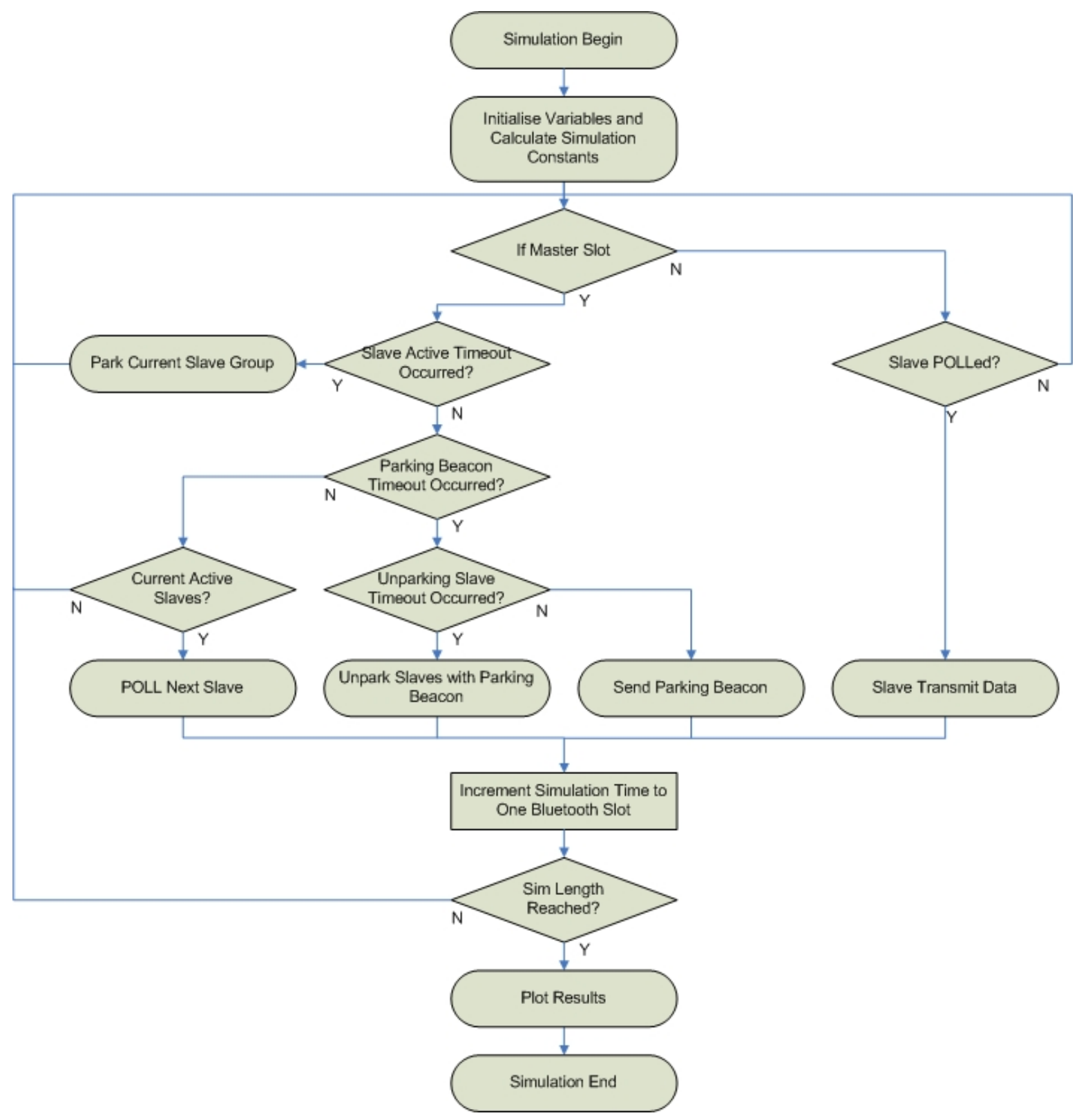

Figure 28: Bluetooth Piconet Sharing Simulation Main Flow Diagram 

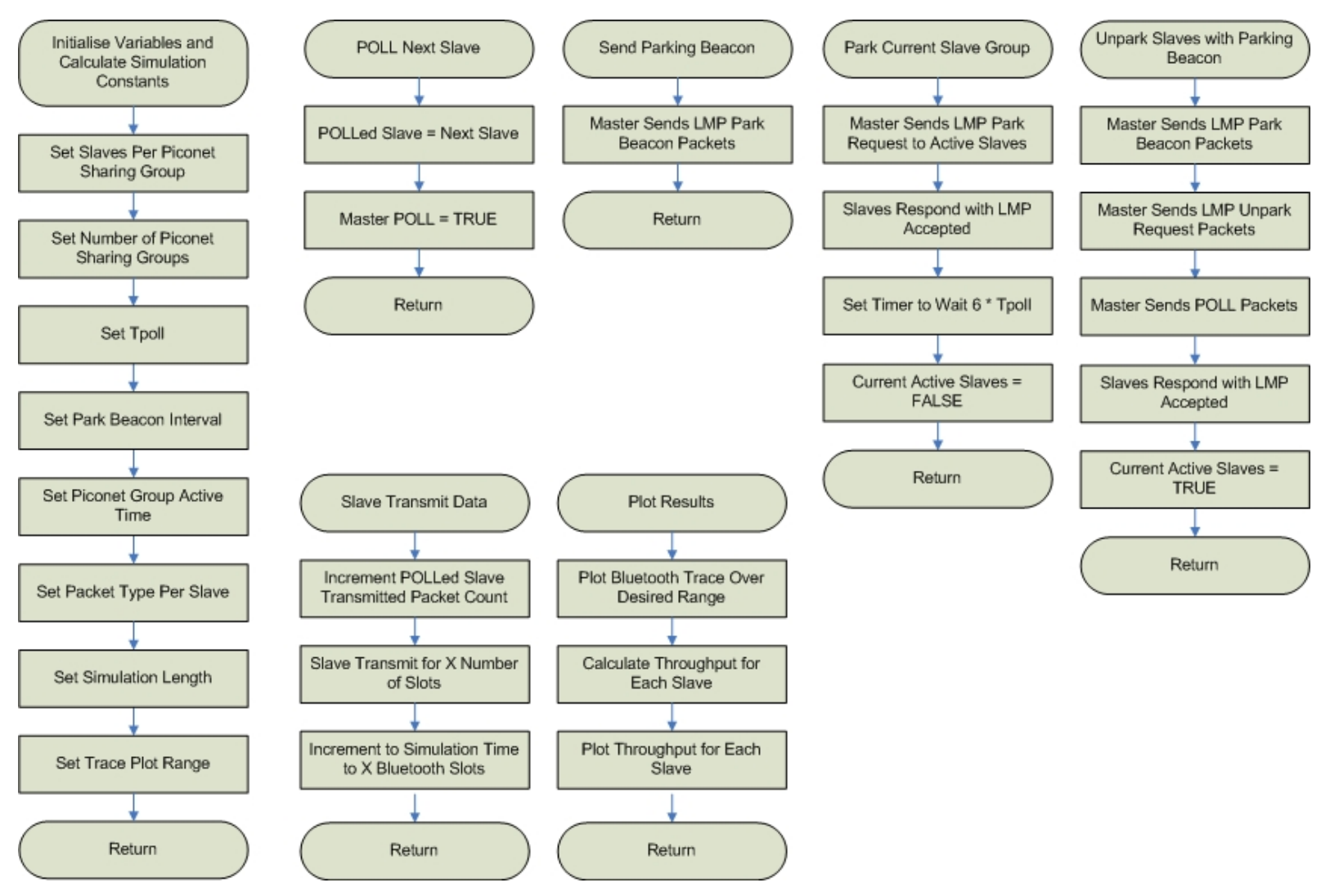

Figure 29: Bluetooth Piconet Sharing Simulation Flow Diagram 


\begin{tabular}{|c|c|c|}
\hline Simulation Parameter & Description (units) & Default Value \\
\hline no_slots & Length of Simulation (slots) & 48000 \\
\hline Tbeacon & Park Beacon Interval (slots) & 2048 \\
\hline active_time & $\begin{array}{l}\text { Time Slaves are active for } \\
\text { (slots) }\end{array}$ & 3746 \\
\hline Tpoll & $\begin{array}{l}\text { Maximum time between } \\
\text { subsequent transmissions } \\
\text { between a master and a slave } \\
\text { (slots) }\end{array}$ & 50 \\
\hline no_slaves_per_swap_group & $\begin{array}{l}\text { Number of Slaves per Piconet } \\
\text { Sharing Swap Group (devices) }\end{array}$ & 5 \\
\hline no_of_swap_groups & $\begin{array}{l}\text { Number of Piconet Sharing } \\
\text { Swap Groups (groups) }\end{array}$ & 2 \\
\hline slave_packet_type $(X)$ & $\begin{array}{l}\text { ACL Packet Type Transmitted } \\
\text { by Slave X (ACL Packet Type) }\end{array}$ & $\begin{array}{l}\text { slave_packet_type(1)= DH5 } \\
\text { slave_packet_type(2)= DH5 } \\
\text { slave_packet_type(3)= DH5 } \\
\text { slave_packet_type(4)= DH5 } \\
\text { slave_packet_type(5)= DH5 } \\
\text { slave_packet_type(6)= DH5 } \\
\text { slave_packet_type(7)= DH5 } \\
\text { slave_packet_type(8)= DH5 } \\
\text { slave_packet_type(9)= DH5 } \\
\text { slave_packet_type(10)= DH5 }\end{array}$ \\
\hline no_piconets & $\begin{array}{l}\text { Number of Coexisting Piconets } \\
\text { (Piconets) }\end{array}$ & 4 \\
\hline bt_trace_min & $\begin{array}{l}\text { Minimum Time Value for the } \\
\text { Bluetooth Trace Output (slots) }\end{array}$ & 3700 \\
\hline bt_trace_max & $\begin{array}{l}\text { Maximum Time Value for the } \\
\text { Bluetooth Trace Output (slots) }\end{array}$ & 3780 \\
\hline
\end{tabular}

Table 10: Bluetooth Piconet Sharing Simulation Parameters

To verify that the Bluetooth park, unpark and park beacon were correctly implemented to match the Bluetooth specification, Bluetooth traces were created to observe these transitional moments in the Bluetooth Piconet sharing structures operation. Figure 30, Figure 31 and Figure 32 show the output Bluetooth traces for the simulation operation. 


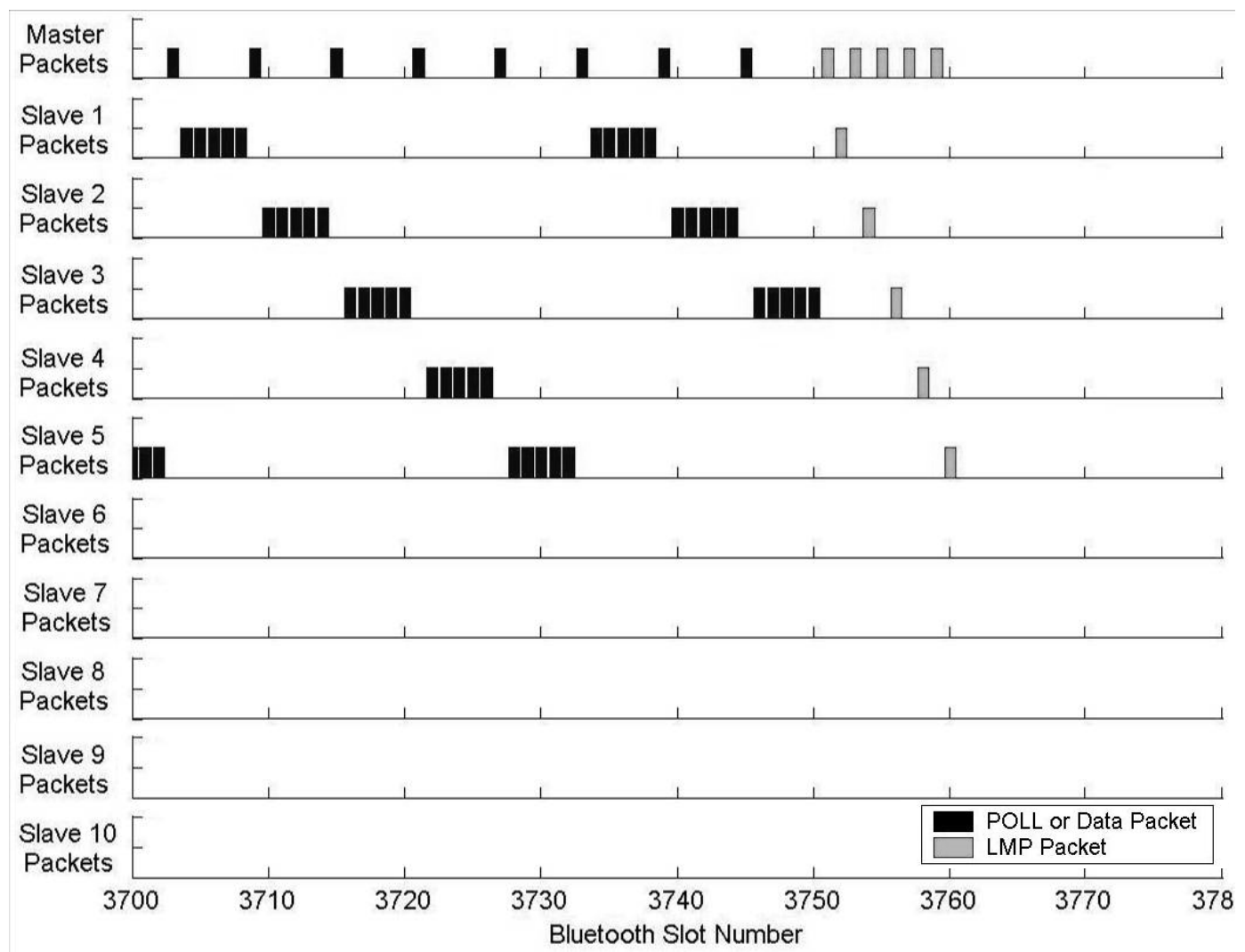

Figure 30: Sample Piconet Sharing Bluetooth Trace Output for Parking Devices

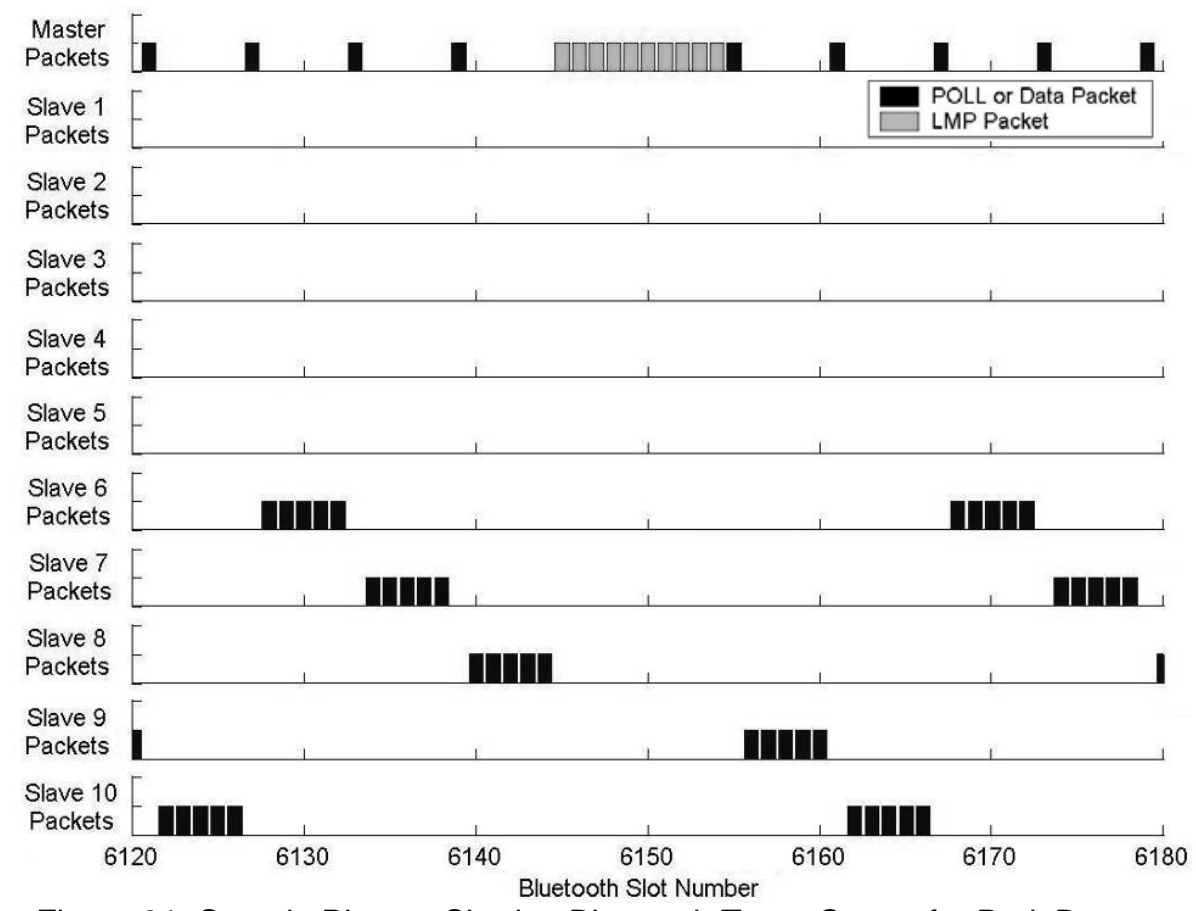

Figure 31: Sample Piconet Sharing Bluetooth Trace Output for Park Beacon - 63 - 


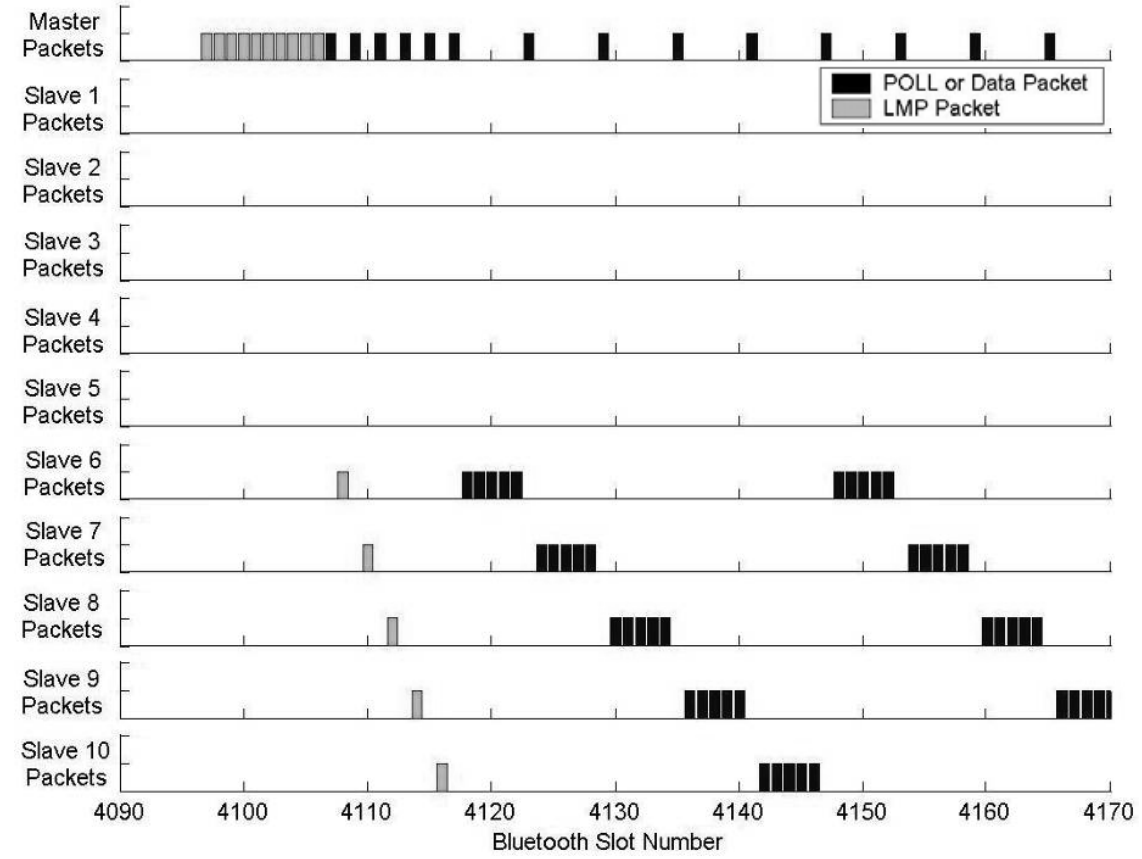

Figure 32: Sample Piconet Sharing Bluetooth Trace Output for Unparking Devices with Parking Beacon The simulated average throughput per Bluetooth device for the example of Piconet sharing ten Bluetooth slaves with two Piconet sharing groups is shown in Figure 33.

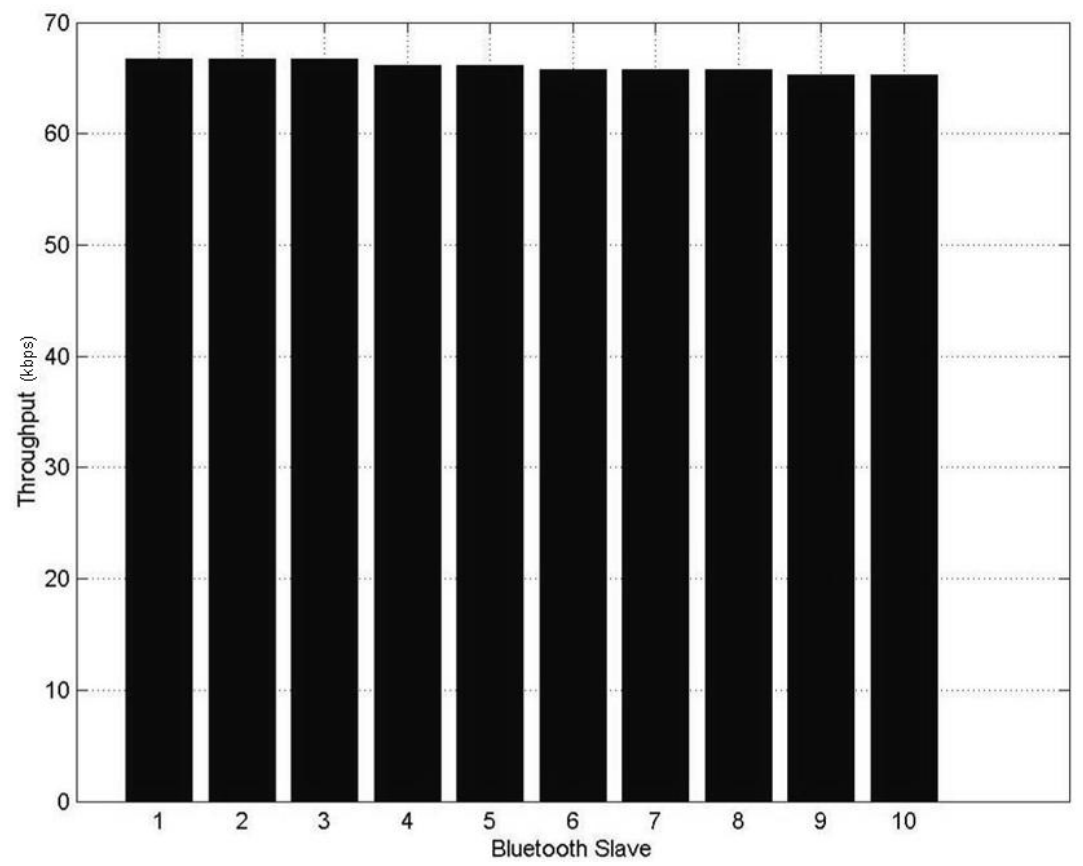

Figure 33: Sample Piconet Sharing Simulation Throughput per Slave Output 
If the active time of each Piconet swap group is selected carefully the devices will be parked just before a park beacon occurs, allowing the next group of devices to be unparked. The active time must be selected to make sure that the Bluetooth channel remains idle for the smallest possible time (allowing the highest average throughput). Figure 34 and Figure 35 show the average throughput results for the Bluetooth Piconet Sharing Structure. The peak throughput for each device can be taken from 4.1.1 Standard Piconet Bluetooth Network Structure Simulation.

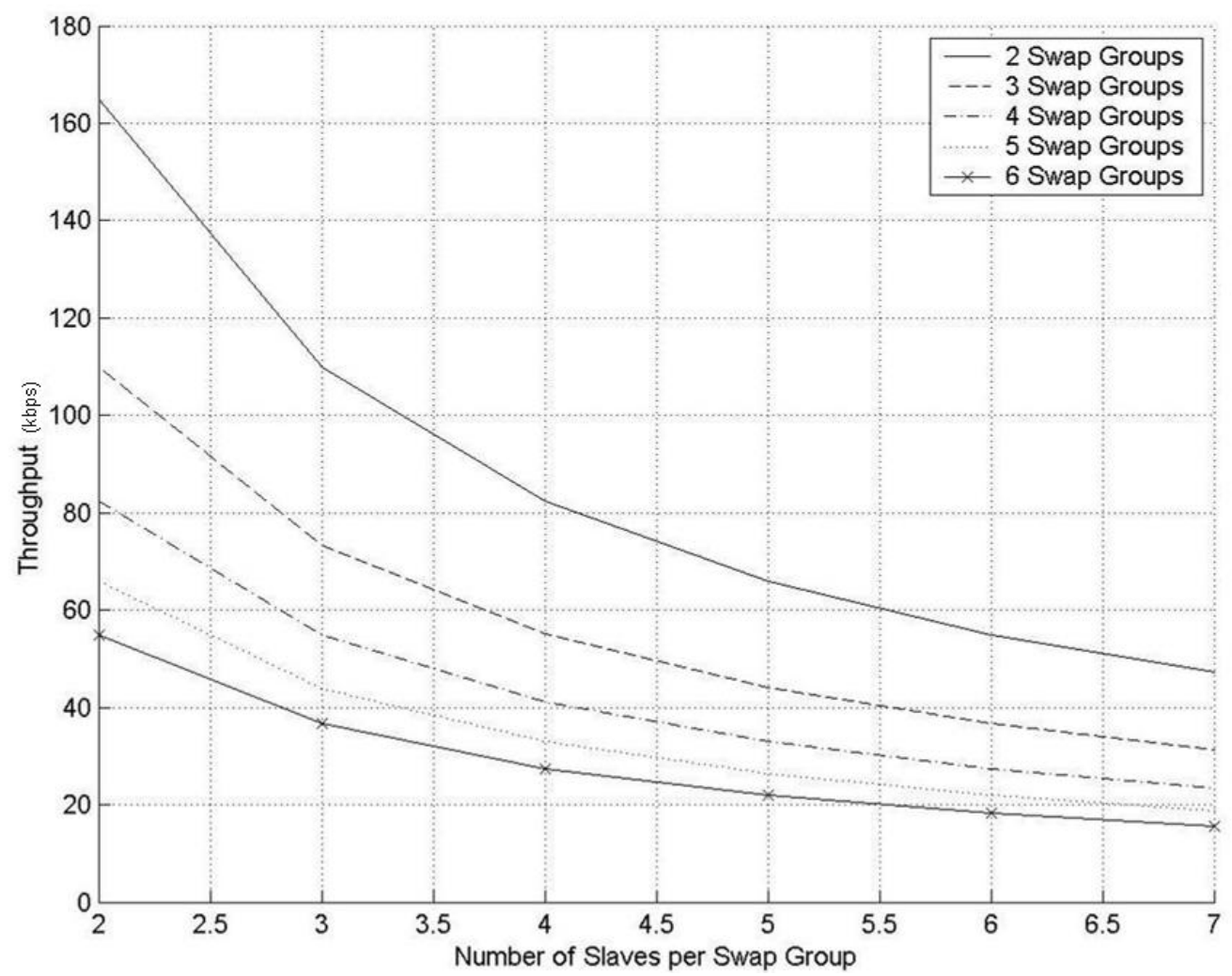

Figure 34: Average Throughput Results for Bluetooth Piconet Sharing Simulation for Differing Numbers of Slaves per Group 


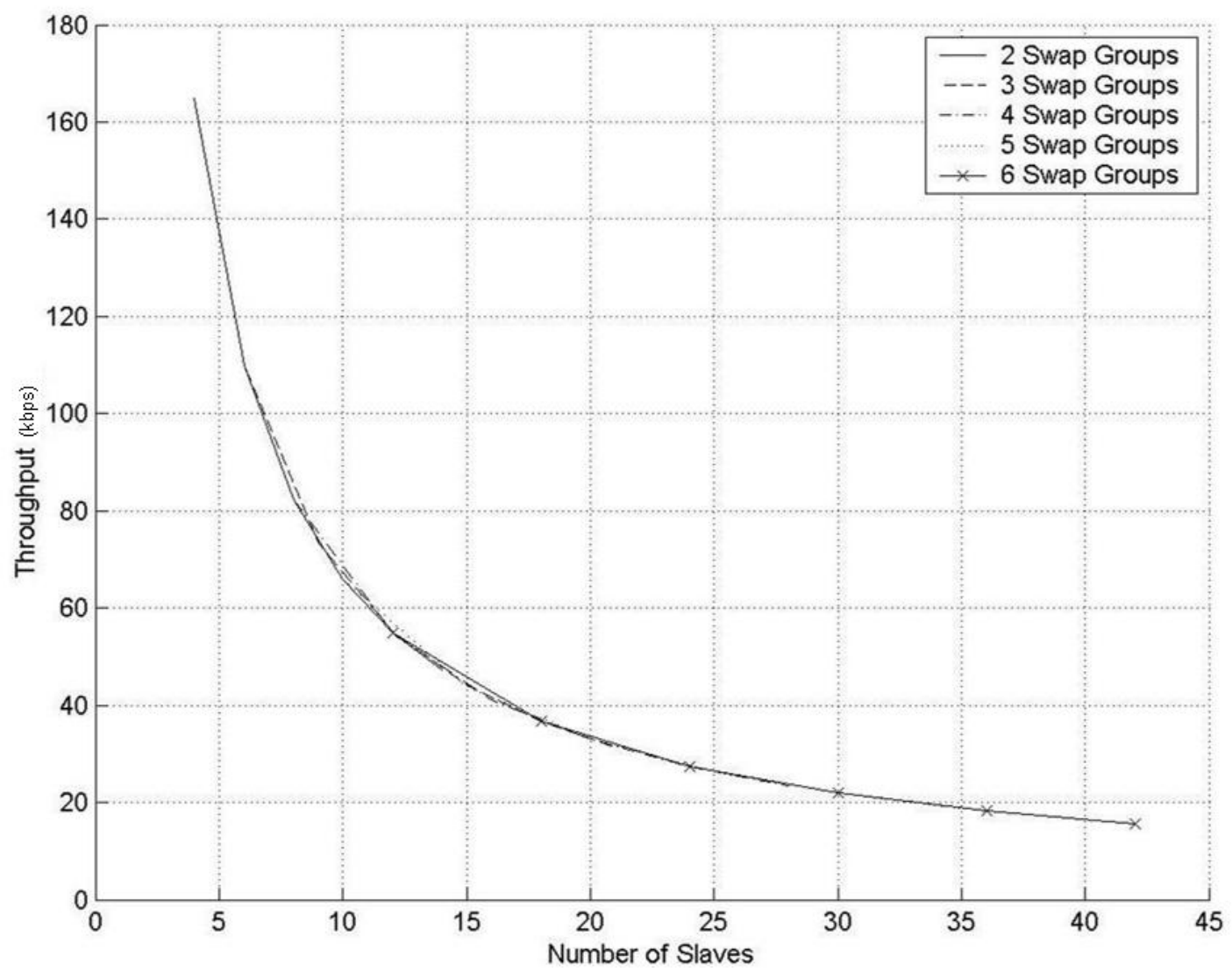

Figure 35: Average Throughput Results for Bluetooth Piconet Sharing Simulation

\subsubsection{Scatternet Tree Bluetooth Network Structure Simulation}

A Bluetooth Scatternet is formed by connecting multiple Piconets together using bridging nodes (see 3.2.3 Scatternet Tree Bluetooth Network Structure). Bridging nodes time share their presence in multiple Piconets, either acting as a slave in both Piconets or acting as a master in one Piconet and a slave in another. The Scatternet Tree Network Structure was built using master / slave bridging nodes only. This allowed for easy routing of the wireless sensor data to the fixed Bluetooth Access point. The Bluetooth specification suggests that the bridging nodes time sharing can be achieved by using the Hold, Park or Sniff Bluetooth modes. Both Hold and Park modes require LMP packets to be sent back and forth to initiate the mode. If the device is placed in sniff mode and the sniff length is chosen correctly, the device can have time to visit another Piconet before the next sniff slot. Figure 36 demonstrates a Bluetooth sniff slot. Dashed lines represent receive packet slots where a packet with matching AM_ADDR field was not received and solid lines represent when one was received. 


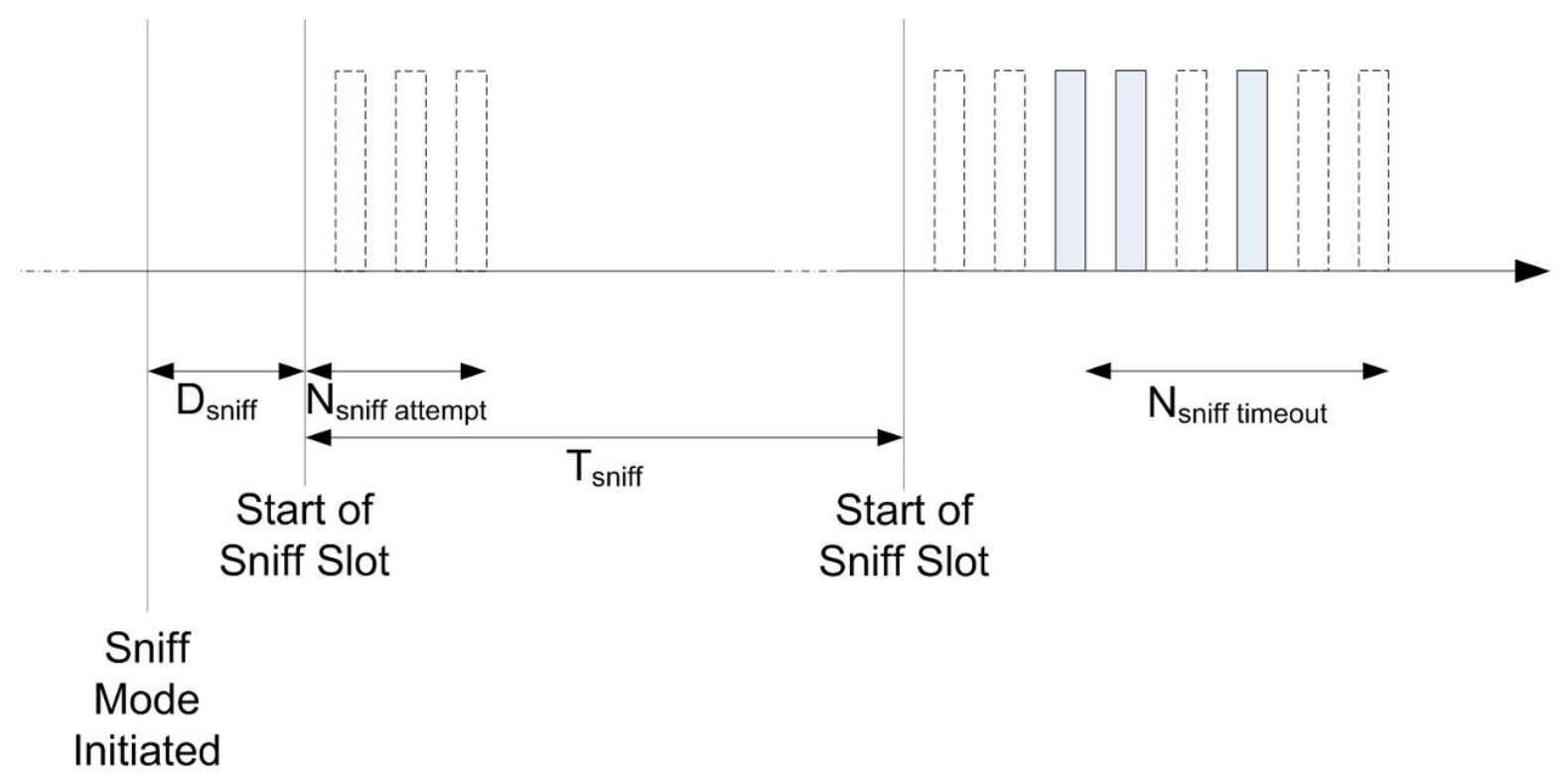

Figure 36: Bluetooth Sniff Mode Sniff Slot [1]

Sniff mode reduces the time the slave is listening to the Piconet. When a slave is placed into sniff mode it is passed the time offset before the first sniff slot $\left(D_{\text {sniff }}\right)$, the interval between sniff slots $\left(T_{\text {sniff }}\right)$, the number of receive slots to wait for a data packet $\left(\mathrm{N}_{\text {sniff attempt }}\right)$ and the number of receive slots until the sniff interval times out $\left(\mathrm{N}_{\text {sniff timeout }}\right)$. When a sniff interval occurs the slave listens for $\mathrm{N}_{\text {sniff attempt }}$ for a packet to be received with a matching AM_ADDR. If a packet is not received the slave goes back into a low power state and awaits the next sniff slot. If a matching packet is received the slave continues listening for $\mathrm{N}_{\text {sniff }}$ timeout or $\mathrm{N}_{\text {sniff attempt }}$ (which ever is larger). An appropriate value of $\mathrm{T}_{\text {sniff }}$ and $\mathrm{N}_{\text {sniff timeout }}$ must be chosen to ensure that the bridging node is in each of the Piconets for the optimum time to ensure the highest possible throughput through the bridging node.

The following limitations and assumptions have been made in this Bluetooth Scatternet Tree Structure Simulation:

- The Bluetooth communication link was never lost.

This simulation was designed to calculate the maximum theoretical throughput of the Bluetooth Scatternet Tree Structure. Inquiry, Paging and connection loss were not included.

- There was no noise or interference.

The simulation assumes a perfect environment where path loss is zero and there are no interfering non-Bluetooth devices. 
- Interference from coexisting Piconet was not included.

The Scatternet Tree Structure contained multiple Piconets connected together to form a Scatternet. These coexisting Piconets will interfere with each other causing a reduction in throughput. This interference was not included in the simulation to keep the simulation complexity to a minimum. The effect of coexisting Piconets was examined in the simulation presented in 4.1.2 Bluetooth Piconet Coexistence Simulation.

- Data was only generated by slaves on the Piconet.

The master only transmits POLL packets to each of the connected slaves.

- Bridging Node Piconet switching time was constant.

In a physical device it takes time for the bridging node to switch between Piconets. This value is dependant on the hardware used and was assumed in the simulation to be six slots (3.75 ms). This value was able to be adjusted in the simulation to more accurately reflect the utilised hardware. The simulation required that this switching delay be an even number of slots.

- Data generated by slaves was at the maximum rate and was sent using DH5 packets.

In this simulation a DH5 packet of data was generated by each slave every time it is polled by the corresponding Master / Slave bridging node. As the Master / Slave bridging node was also a wireless sensing device it also generated data at the same rate as the slaves.

- The Bluetooth Scatternet Tree was limited to a maximum of 2 Scatternet levels.

Limiting the number of Scatternet levels in the simulation greatly decreased the implementation complexity. Limiting the Scatternet levels to 2 limited the maximum number of sensor devices in this structure to 56 .

- Each Piconet in the Simulation contained the same number of slaves.

Imposing this limitation on the simulation further reduces the simulations complexity.

The flow charts for the implemented Bluetooth Scatternet Tree Structure simulation is given in Figure 37 and Figure 38. The Scatternet Tree Structure Simulation code can be found in Appendix D. The simulation parameters for the Scatternet Tree Structure are summarised in Table 11. 


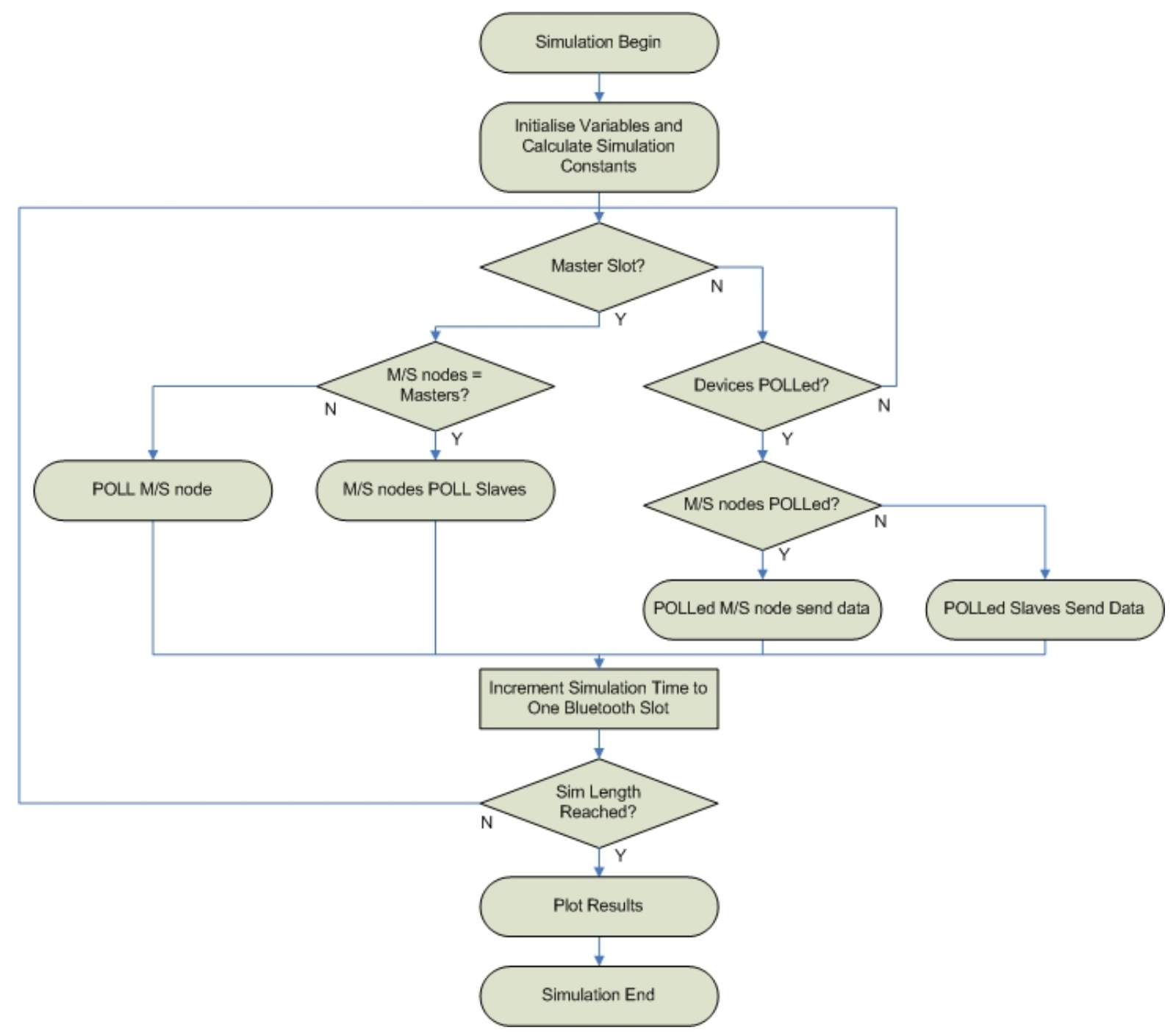

Figure 37: Bluetooth Scatternet Tree Structure Simulation Main Flow Diagram 

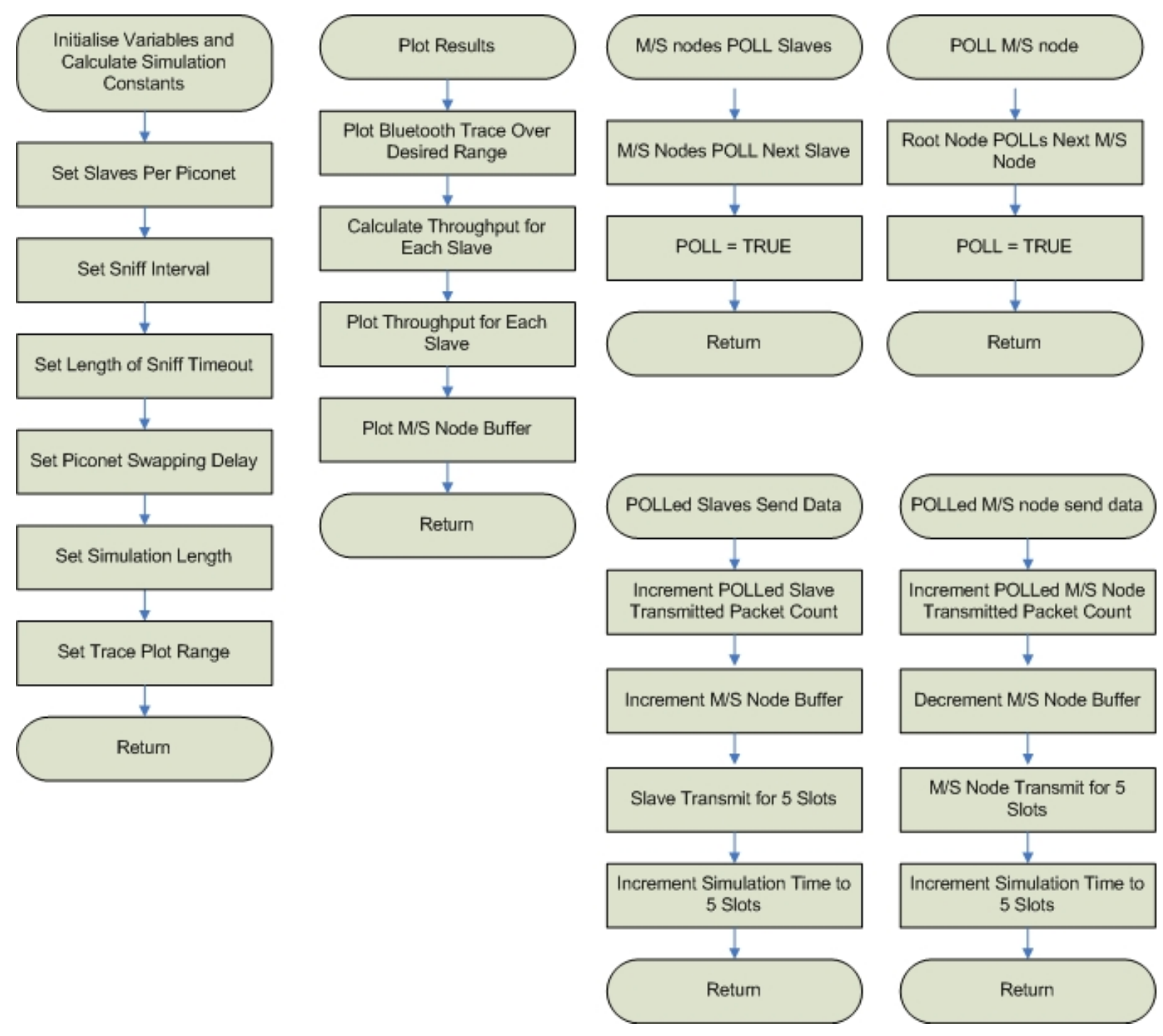

Figure 38: Bluetooth Scatternet Tree Structure Simulation Flow Diagram 


\begin{tabular}{|c|c|c|}
\hline Simulation Parameter & Description (units) & Default Value \\
\hline no_slots & Length of Simulation (slots) & 48000 \\
\hline no_slaves_per_piconet & $\begin{array}{l}\text { Number of Slaves per Piconet } \\
\text { (devices) }\end{array}$ & 3 \\
\hline no_of_levels & $\begin{array}{l}\text { Number of Scatternet Levels } \\
\text { (levels) }\end{array}$ & $\begin{array}{l}2 \text { (this value must be kept as } \\
\text { 2) }\end{array}$ \\
\hline Tsniff & Sniff mode interval (slots) & 4800 \\
\hline Nsniff_timeout & Sniff mode timeout (slots) & 4100 \\
\hline delay & $\begin{array}{l}\text { Delay when swapping Piconets } \\
\text { (slots) }\end{array}$ & 6 \\
\hline bt_trace_min & $\begin{array}{l}\text { Minimum Time Value for the } \\
\text { Bluetooth Trace Output (slots) }\end{array}$ & 3800 \\
\hline bt_trace_max & $\begin{array}{l}\text { Maximum Time Value for the } \\
\text { Bluetooth Trace Output (slots) }\end{array}$ & 3880 \\
\hline
\end{tabular}

Table 11: Bluetooth Scatternet Tree Simulation Parameters

A sample Bluetooth trace is given in Figure 39. In this example a 2 level Scatternet was simulated with three slaves per Piconet. This meant that the simulation contained 3 Master / Slave bridging nodes and 9 slave nodes. The light coloured packets in the Bluetooth trace represent POLL packets from the Piconet master and the dark represent data packets. This Bluetooth trace shows the transition of the bridging nodes from participating as Slaves in the Bluetooth access point Piconet to acting as Masters of the Slave devices. 


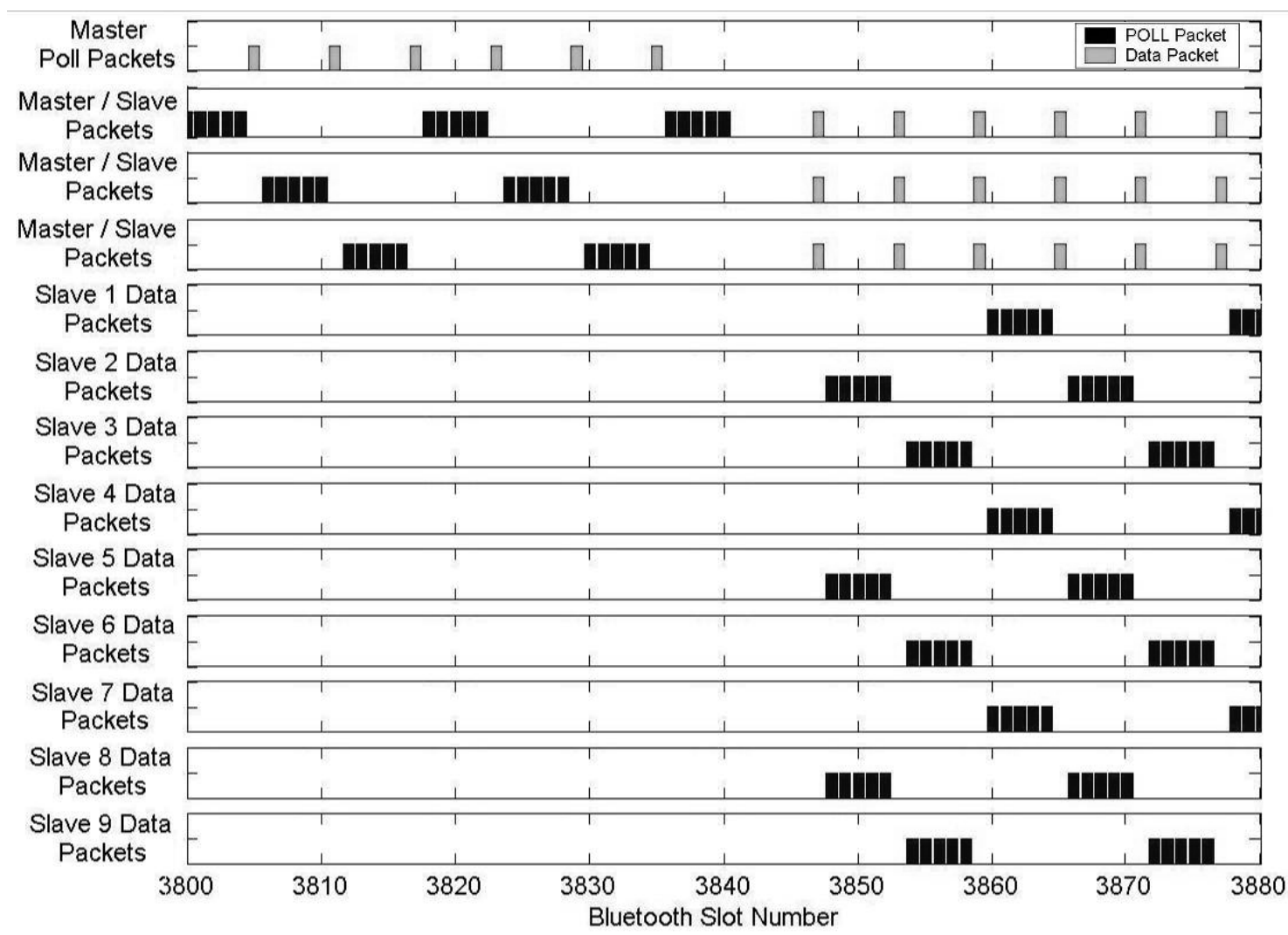

Figure 39: Example Scatternet Tree Bluetooth Trace Output

The optimum sniff length occurs when the bridging node has exactly the correct length of time in the root nodes Piconet to transmit the data that is stored in the data buffer. Equation 2 represents the optimum value for sniff timeout that leads to the highest throughput through the Master / Slave bridging node.

$$
N_{\text {sniff timeout }}=T_{\text {sniff }} \times\left(\frac{N_{\text {slaves per piconet }}+1}{N_{\text {slaves per piconet }}+2}\right)
$$

Where:

$$
\begin{array}{ll}
N_{\text {sniff timeout }} & =\text { Number of receive slots to listen for a matching packet after } \\
& \text { initial received packet } \\
T_{\text {sniff }} & =\text { Interval between sniff slots } \\
N_{\text {slaves per piconet }} & =\text { Number of slaves per Piconet }
\end{array}
$$

Using Equation 2 the optimum sniff timeout for the sample Scatternet Tree (3 slaves per Piconet) was calculated to be 3840 slots (2.4 seconds) when using a sniff interval of 4800 slots (3 seconds). This ensured that the bridging nodes had enough time to transmit all the data that had been buffered from the 
slave nodes and from the attached wireless sensor platform. Figure 40 shows the throughput per node output and data buffer for the optimum sniff length.

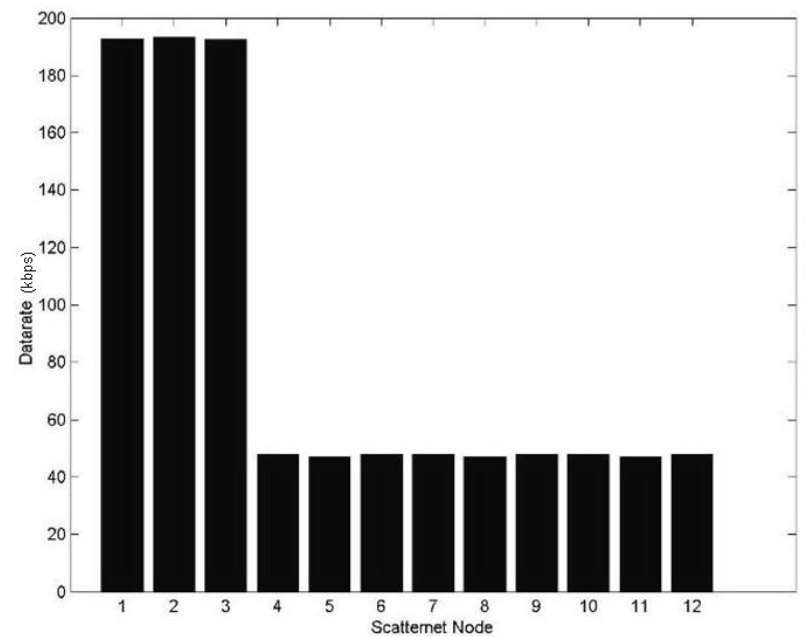

(a)

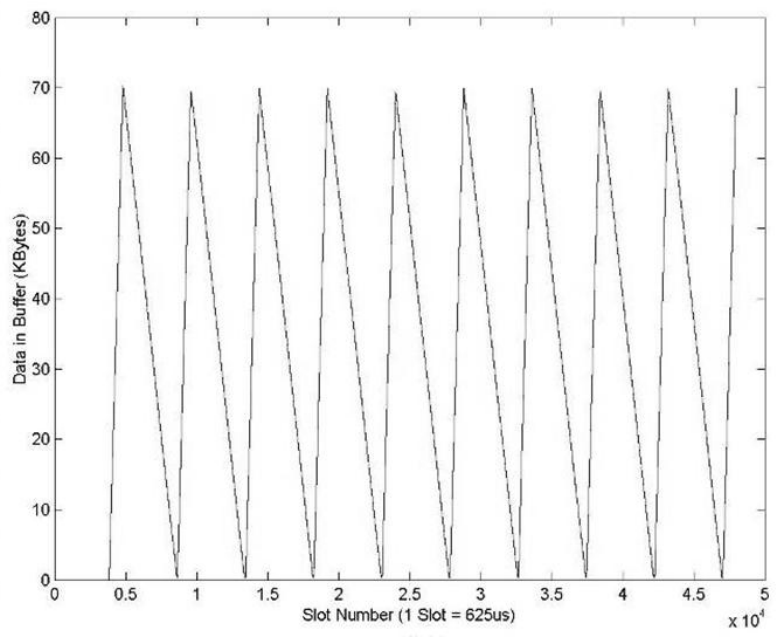

(b)

Figure 40: Example Bluetooth Scatternet Tree Structure with Optimum Sniff Timeout;

(a) Throughput per Node Output and (b) Master / Slave Node Data Buffer

If the sniff time out was too high the bridging node will stay in the root nodes Piconet too long resulting in a lower throughput for the slave devices. This can be seen in Figure 41 as the throughput for the slaves decreased and the data buffer was left empty for large periods of time. In this example the sniff timeout was set to 4100 slots (2.5625 seconds).

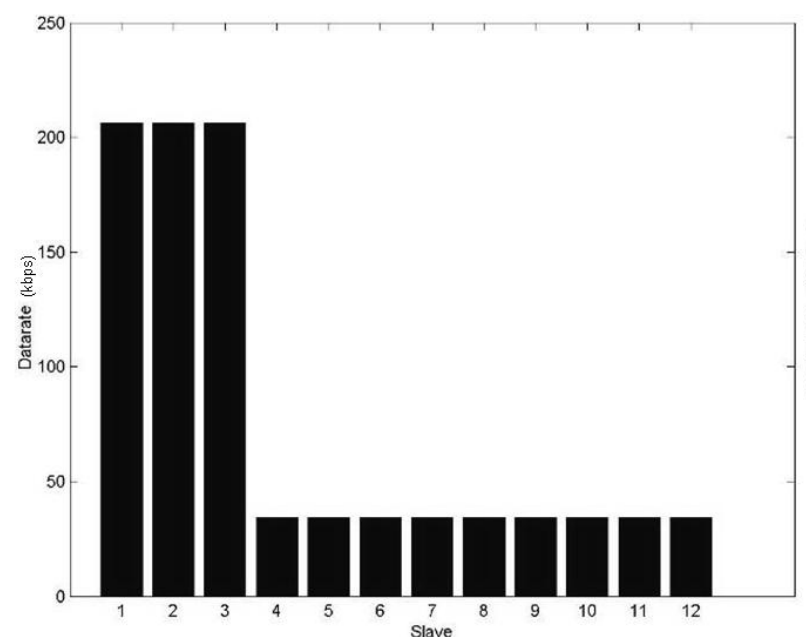

(a)

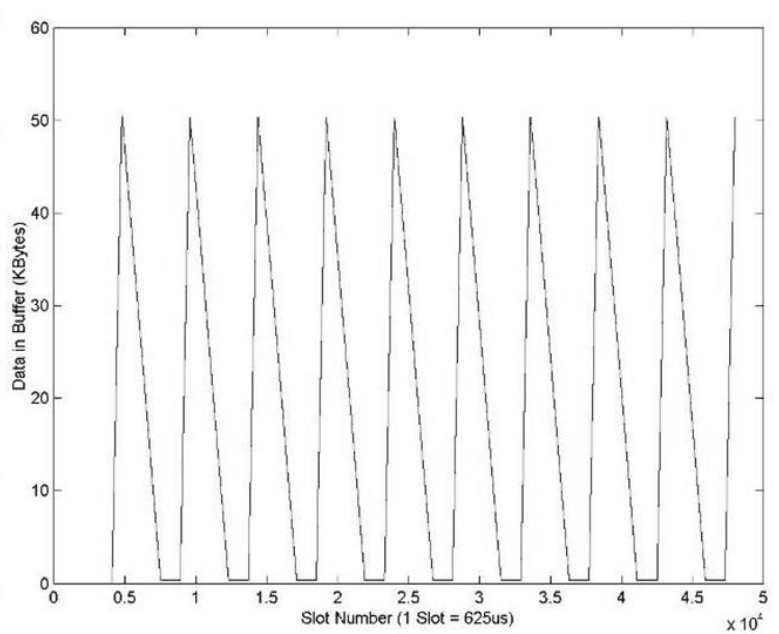

(b)

Figure 41: Example Bluetooth Scatternet Tree Structure with Too Large Sniff Timeout;

(a) Throughput per Node Output and (b) Master / Slave Node Data Buffer -73 - 
When the sniff timeout was set too low the bridging nodes do not spend long enough in the root node Piconet and do not empty the data buffers. Figure 42 shows this with the data buffer continually increasing causing a buffer overflow when implemented in hardware. This example used a sniff timeout of 3500 slots (2.1875 seconds).

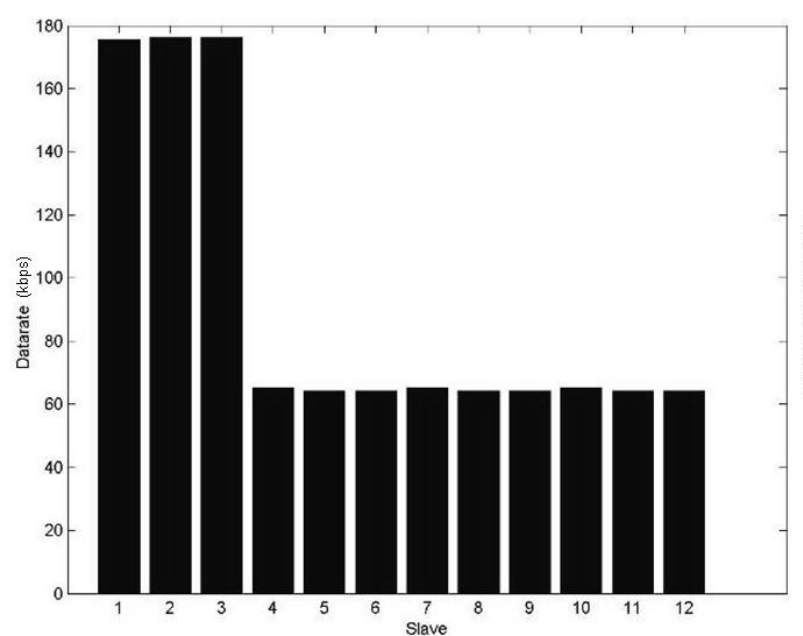

(a)

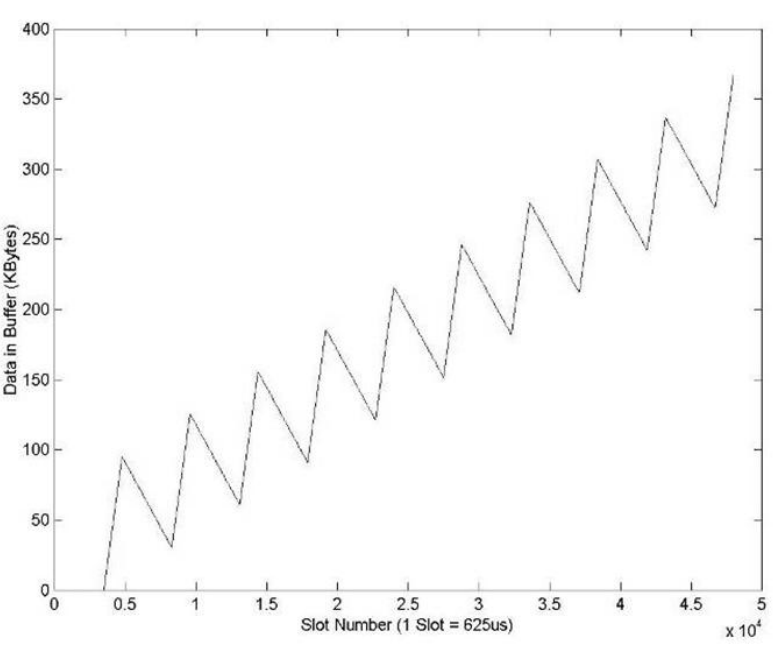

(b)

Figure 42: Example Bluetooth Scatternet Tree Structure with Too Small Sniff Timeout;

(a) Throughput per Node Output and (b) Master / Slave Node Data Buffer

Figure 40 , Figure 41 and Figure 42 highlight the fact that the bridging node in the Scatternet Tree Structure must be allocated enough time to transmit the collected data from the Piconet to the root node. Careful selection of the sniff interval must be undertaken to ensure that a buffer overrun does not occur. When implementing the Scatternet Tree Structure in hardware it was better to err on the side of caution and choose a slightly lower sniff interval than the optimum value. To ensure that the data buffer is emptied on the bridging node an additional software algorithm could be implemented to vary the sniff interval of the bridging node based upon the amount of data in the data buffer.

Figure 43 shows the overall Baseband throughput results for the Bluetooth Scatternet Tree Structure using an optimum sniff time, DH5 packets and a maximum of 2 Scatternet levels (maximum of 56 devices). Table 12 shows the possible node combinations of the Scatternet Tree network structure. The number of coexisting Piconets increases along with the number of sensing devices. The effect of these coexisting Piconets was not taken into consideration in this simulation therefore the network throughput observed in practice will be reduced. 


\begin{tabular}{|l|l|l|}
$\begin{array}{l}\text { Number of } \\
\text { Slaves Per } \\
\text { Piconet }\end{array}$ & $\begin{array}{l}\text { Number of } \\
\text { Coexisting } \\
\text { Piconets }\end{array}$ & $\begin{array}{l}\text { Total Number of Sensing } \\
\text { Devices }\end{array}$ \\
\hline 2 & 3 & 6 \\
\hline 3 & 4 & 12 \\
\hline 4 & 5 & 20 \\
\hline 5 & 6 & 30 \\
\hline 6 & 7 & 42 \\
\hline 7 & 8 & 56 \\
\hline
\end{tabular}

Table 12: Bluetooth Scatternet Tree Network Structure Possible Node Combinations

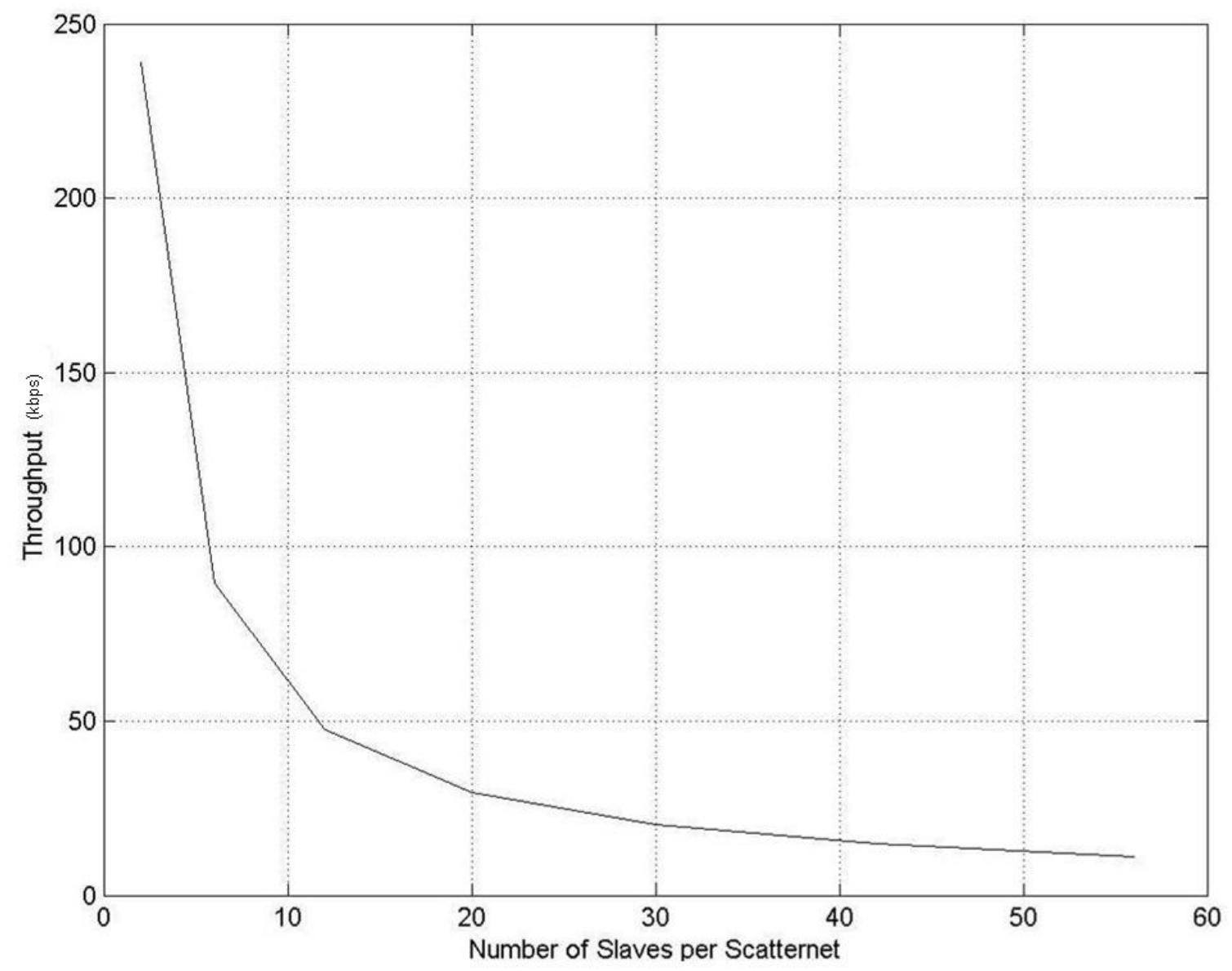

Figure 43: Average Throughput Results for Bluetooth Scatternet Tree Structure Simulation 


\subsubsection{Piconet Tree Bluetooth Network Structure Analysis}

A Piconet Tree is formed by using bridging nodes (that consist of multiple Bluetooth radios) and sensing nodes (that consist of Bluetooth radios and sensor platforms) to form a tree structure (outlined in 3.2.4 Piconet Tree Bluetooth Network Structure). The benefit of using a Piconet based tree structure compared with a Scatternet tree structure is that each Bluetooth radio only communicates in a single Piconet and so the network structure is a lot less resource intensive. The draw back is that bridging nodes only have data routing functionality and cannot support a sensor platform. As this simulation consists of coexisting Piconets the simulation presented in 4.1.2 Bluetooth Piconet Coexistence Simulation has been used to analyse this structure. Table 13 shows the different combinations of devices for this structure (limited to two tree levels). Note that the number of actual sensing devices for the Piconet tree network structure is less than the total number of devices in the structure due to the non sensing bridging nodes.

\begin{tabular}{|l|l|l|l|}
$\begin{array}{l}\text { Number of } \\
\text { Slaves Per } \\
\text { Piconet }\end{array}$ & $\begin{array}{l}\text { Number of } \\
\text { Coexisting } \\
\text { Piconets }\end{array}$ & $\begin{array}{l}\text { Total Number of } \\
\text { Devices (including } \\
\text { bridging nodes) }\end{array}$ & $\begin{array}{l}\text { Total Number of Sensing } \\
\text { Devices (not including } \\
\text { bridging nodes) }\end{array}$ \\
\hline 2 & 3 & 6 & 4 \\
\hline 3 & 4 & 12 & 9 \\
\hline 4 & 5 & 20 & 16 \\
\hline 5 & 6 & 30 & 25 \\
\hline 6 & 7 & 42 & 36 \\
\hline 7 & 8 & 56 & 49 \\
\hline
\end{tabular}

Table 13: Bluetooth Piconet Tree Network Structure Possible Node Combinations

Entering the number of coexisting Piconets from Table 13 into the Piconet Coexistence Simulation the network throughput simulated the Piconet Tree Bluetooth Structure. The network throughput results are given in Figure 44. 


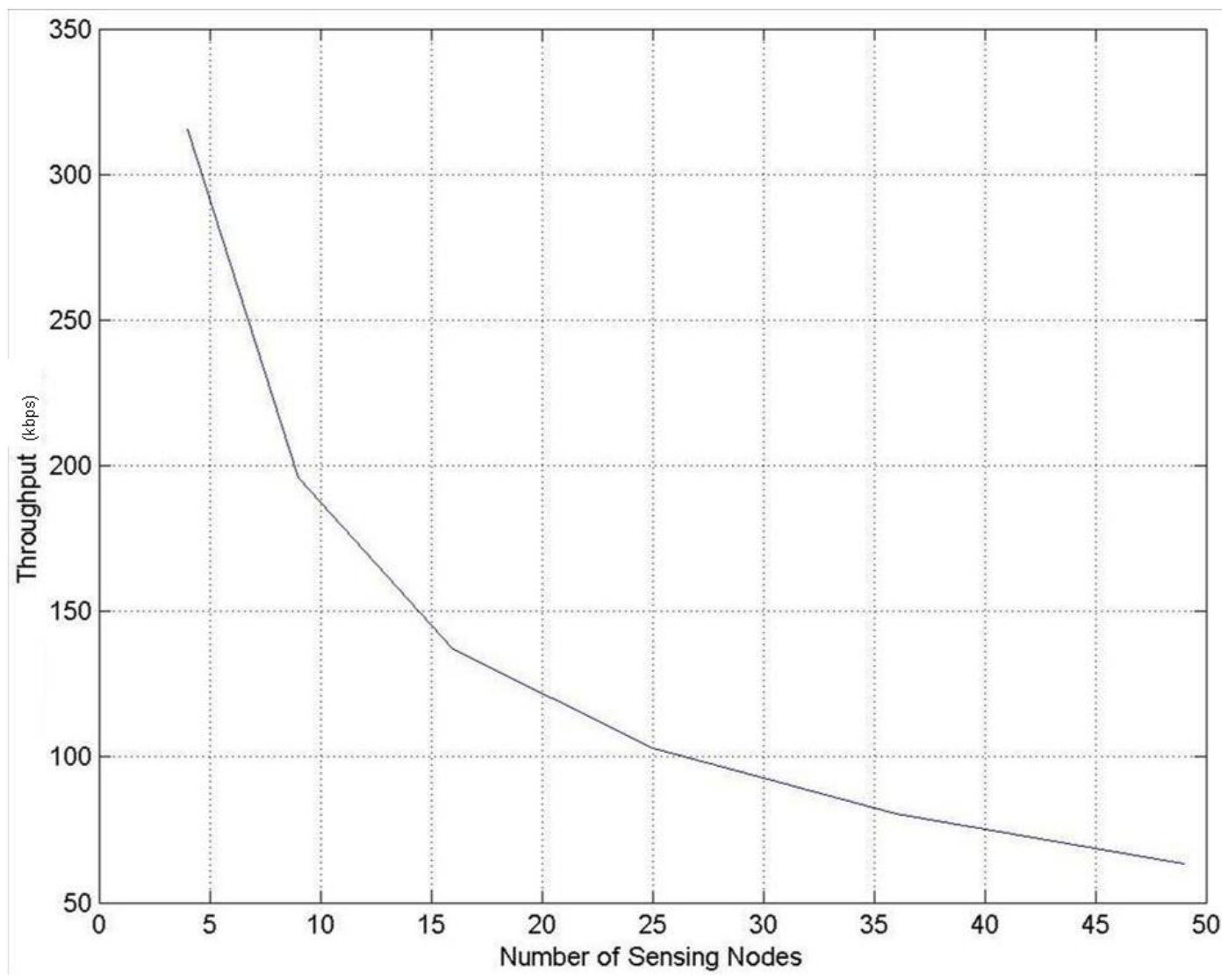

Figure 44: Throughput Results for Bluetooth Piconet Tree Network Structure Simulation

\subsubsection{Multiple Piconet Bluetooth Network Structure Analysis}

The Multiple Piconet Bluetooth Network Structure was created by adding multiple Bluetooth radios to the Bluetooth access point in each wireless cell. This allowed up to seven active slaves per Piconet and higher overall throughput compared with a single Bluetooth radio. This scenario was again made up of coexisting Piconets so the simulation presented in 4.1.2 Bluetooth Piconet Coexistence Simulation was used. Table 14 shows the combination of nodes possible in the Multiple Piconet Bluetooth Network Structure. 


\begin{tabular}{|l|l|l|}
$\begin{array}{l}\text { Number of } \\
\text { Slaves Per } \\
\text { Piconet }\end{array}$ & $\begin{array}{l}\text { Number of } \\
\text { Coexisting } \\
\text { Piconets }\end{array}$ & $\begin{array}{l}\text { Total Number } \\
\text { of Sensing } \\
\text { Devices }\end{array}$ \\
\hline 1 & 4 & 4 \\
\hline 2 & 4 & 8 \\
\hline 3 & 4 & 12 \\
\hline 4 & 4 & 16 \\
\hline 5 & 4 & 20 \\
\hline 6 & 4 & 24 \\
\hline 7 & 4 & 28 \\
\hline
\end{tabular}

Table 14: Bluetooth Multiple Piconet Network Structure Possible Node Combinations

Using the coexisting Piconet simulation (with four coexisting picoents) the network throughput results are shown as Figure 45.

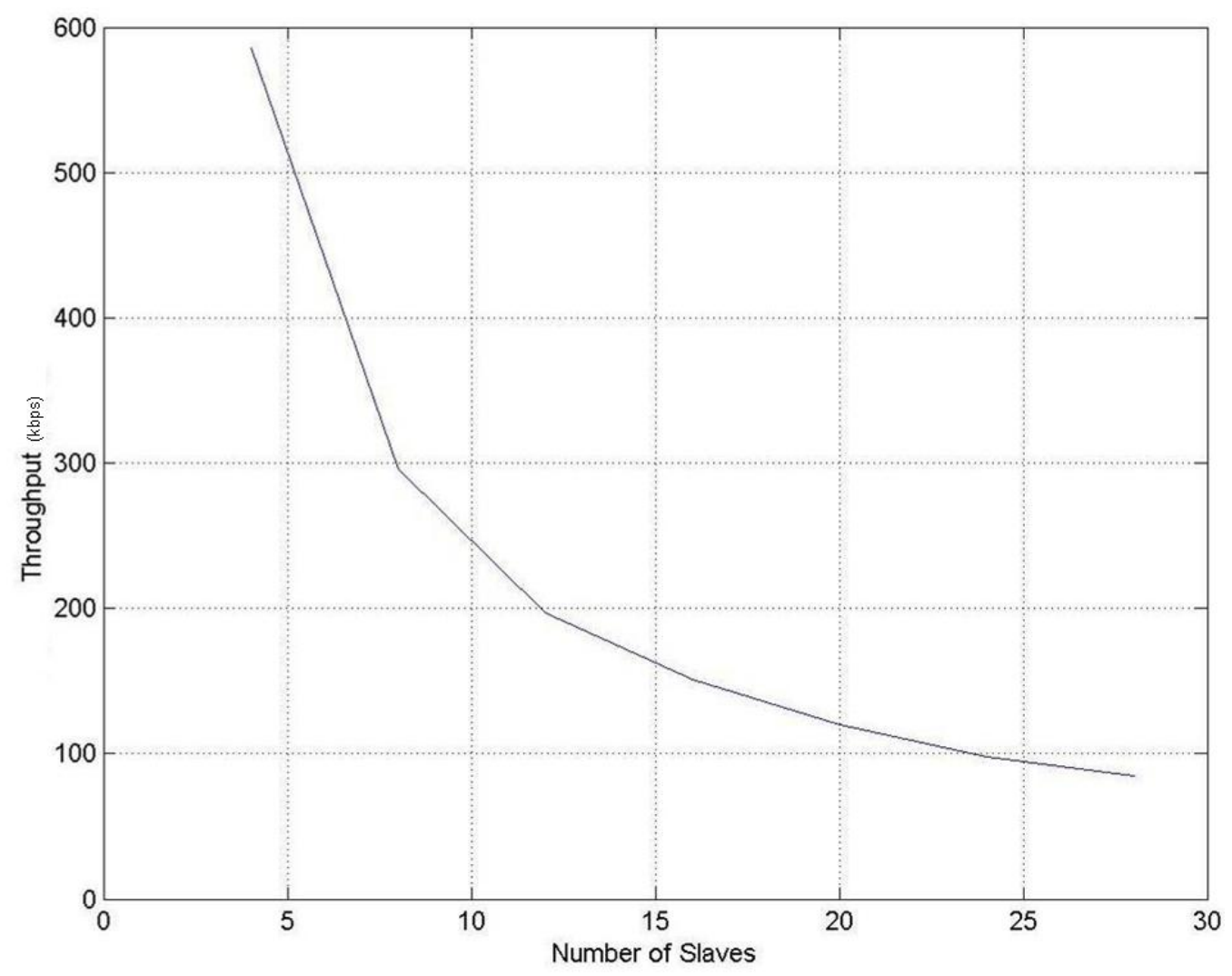

Figure 45: Throughput Results for Multiple Piconet Network Structure Simulation 
As expected the Multiple Piconet Network Structure produced the highest throughput per slave results. These throughput results would be increased further if additional Bluetooth radios were implemented in the Bluetooth access point root node. Increasing the number of Bluetooth radios increases the number of connectable Bluetooth devices, however will result in a higher number of coexisting Piconets.

\subsection{Bluetooth Wireless Sensor Network Simulation Summary}

Four Bluetooth simulation tools have been written in MATLAB to give the theoretical performance of the proposed Bluetooth network structures. These simulation tools have been written in a manner which will make them easily customisable for the analysis of future Bluetooth communication applications.

The Standard Bluetooth Piconet Network Structure Simulation examined Bluetooth communications in a noise free environment to aid in verification of the simulation methodology. The effect of the overall throughput per node on a Bluetooth Piconet can be examined by modifying the number of nodes and Bluetooth packet types.

The Bluetooth Piconet Coexistence Simulation demonstrated the worst case effect of coexisting Bluetooth Piconets. The coexisitng Piconets in this simulation transmitted single ACL packet data (DM1 or DH1), which results in the highest rate of interferance. Simulation parameters such as the number of coexisting picoents and Bluetooth packets types can be modified to examine the effect on the throughput per node. The masters POLLing policy can also be modified to rePOLL a slave if communications were unsuccessful. This had a large effect on the throughput per slave. This simulation demonstrated in an enviroment with a large number of coexisting piconets, transmitting smaller packets with a reduced payload size actually results in a higher throughput per node. This is due to in an environment with a large number of coexisting Piconets, smaller packets having a higher probability of being received.

The Piconet Sharing Bluetooth Network Structure Simulation calculated the theorectical throughput per node for the proposed Piconet Sharing Network Structure. Simulation properties such as number of nodes per swap group, number of swap groups, active time, and park beacon were modified to examine the effect on the throughput. This simulation highlighted the large latency between active communications for each of the slaves.

The Scatternet Tree Bluetooth Network Structure Simulation calculated the theorectical throughput per node of a scatternet tree formed with master / slave bridging nodes. This simulation demonstrated the effect on the Scatternet throughput of the sniff timeout selected. if the value is too large the data buffer of the bridging node remains empty, resulting in a reduced throughput. However If the value of the sniff timeout is too small then the data buffer of the brigding node is never able to empty resulting in a buffer overflow. It was demonstrated that the sniff timeout of the Scatternet Tree bridging nodes should be set based upon the number of slaves per piconet of the Scatternet Tree.

-79 - 
The Piconet Tree Bluetooth Network Structure and the Multiple Piconet Bluetooth Network Structure were anaylised using the Standard Piconet Bluetooth Network Structure Simulation and Bluetooth Piconet Coexistence Simulation tools. All of these simulation tools were specifically written for the developed network structures and represent an original research contribution. These tools allowed theorectical throughput per node values to be obtained for these network structures.

All of the simulated and anaylsised Bluetooth network structures provided the theorectical required throughput per node for the wireless sensing applciation. The proposed network structures were then required to be tested in hardware to investigate the hardware and software limitations of the hardware used. It is not expected that the hardware testing results will be as high as presented in this section due to these hardware limiations. 


\section{Bluetooth Wireless Sensor Network Testing}

This chapter investigates the effectiveness of the developed Bluetooth Network Structures through hardware implementation. Each of the structures proposed in Chapter 3 Bluetooth Wireless Sensor Network Design has been tested using existing Bluetooth hardware and software stack. The test hardware was first tested for conformity to the Bluetooth Specification. Power consumption, connection latencies and range limitations were measured for the wireless sensor nodes. Standard Bluetooth Piconets were constructed and tested with both the root node hardware and wireless sensor node hardware acting as the Piconet master. These tests were undertaken to ensure that up to the maximum of seven slaves could communicate on the Piconet at a time. Each of the proposed Bluetooth network structures was then implemented and tested.

The majority of existing Bluetooth research presents purely simulated results. By implementing each of the developed network structures with available hardware, real world throughput results can be obtained with current devices hardware limitations. Presenting real world hardware testing of standard Bluetooth Piconet structures and the developed network structures is an original contribution to the research field. This real world testing highlighted several limitations with the tested Bluetooth hardware.

The tests described in this section have been undertaken in an anechoic chamber to limit potential interference from other devices. Due to the Bluetooth network structures being at the proof of concept stage, interference from non-Bluetooth devices has been assumed as negligible. The testing location was selected as it is free from all transmissions in the $2.4 \mathrm{GHz}$ ISM band and there are minimal reflections from walls that could possibly cause multi-path effects. Several assumptions have been made when testing each of the network structures, these assumptions have been explained in the relevant sections. These assumptions have allowed the extrapolation of the testing results to demonstrate network throughput at higher node number limits. Limitations in the testing methodology have been highlighted where appropriate.

\section{Test Hardware}

The test hardware used to create the Bluetooth root nodes is made up of an Intrinsic Cerfboard 255 single board computer (SBC) [64] combined with a CC\&C Technologies Bluetooth wireless radio module [65]. The SBC is running Windows Compact Edition (WinCE) 4.2. The CC\&C Bluetooth module contains a Cambridge Silicon Radio (CSR) BlueCore02 (BC02) External Chipset. This chipset can either be flashed with Host Controller Interface $(\mathrm{HCl}$ ) version firmware (Using the WinCE Bluetooth stack as the upper layer protocols) or can be flashed with RFCOMM version firmware (all Bluetooth layers are integrated on the BC02 chip). The SBC has onboard 10/100 Ethernet and this has been used as the test 
high speed data backbone. In practice a high speed non-interfering wireless technology (eg IEEE 802.11a) is a more cost effective and easier solution. The test hardware used in the hardware testing can be seen in Figure 46.

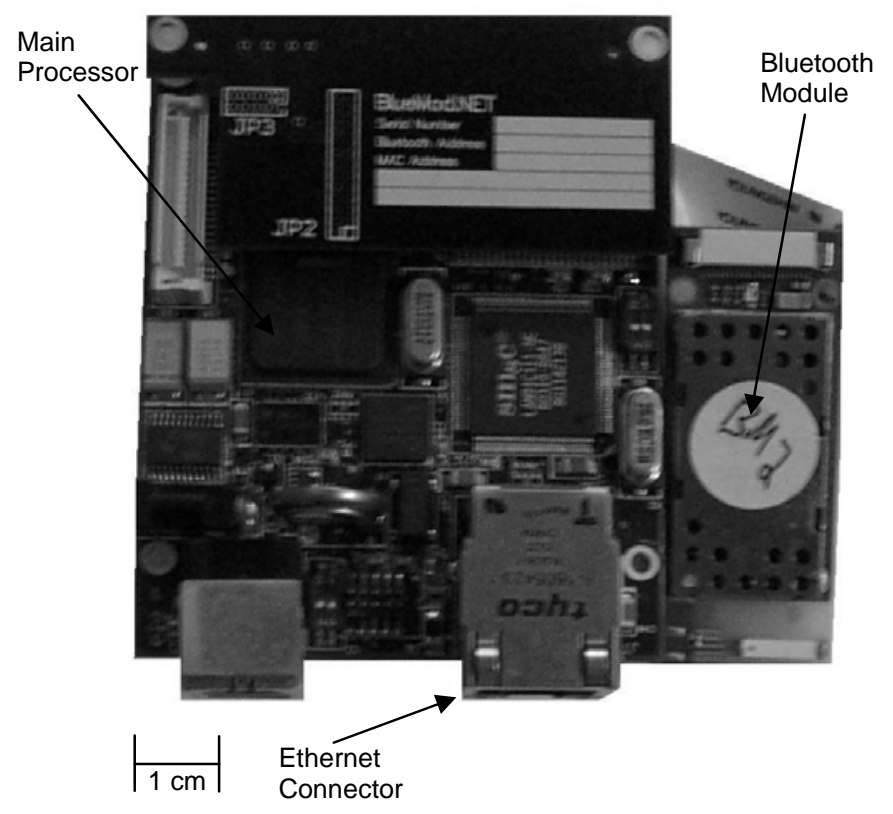

(a)

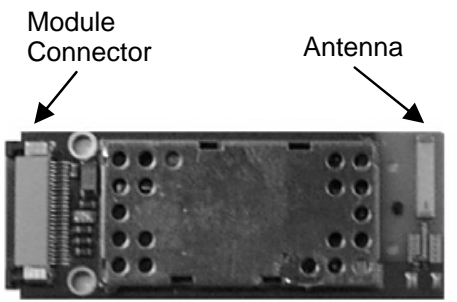

(b)

Figure 46: Bluetooth Hardware Used in Wireless Sensor Testing;

(a) Bluetooth Root Node [64] and (b) Wireless Sensor Node (Radio Only) [65]

The wireless sensor nodes are made up of a CC\&C Bluetooth module and the developed sensor platform (not shown). The BC02 chipsets on the CC\&C modules are flashed with the complete Bluetooth stack and developed network structure and have been designed as simple serial replacement devices to the wireless sensor platform. This allows the developed wireless sensor network to be quickly integrated into existing wireless sensing applications that the current sensor platform is being used for. Figure 47 shows the stack architecture for the Bluetooth root node and wireless sensor node. 


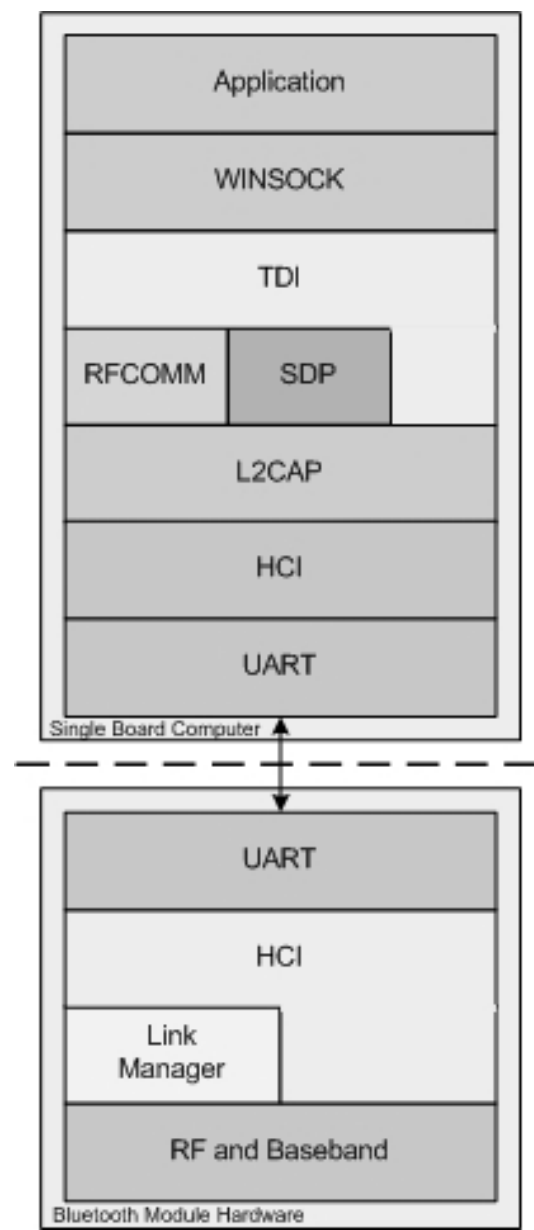

(a)

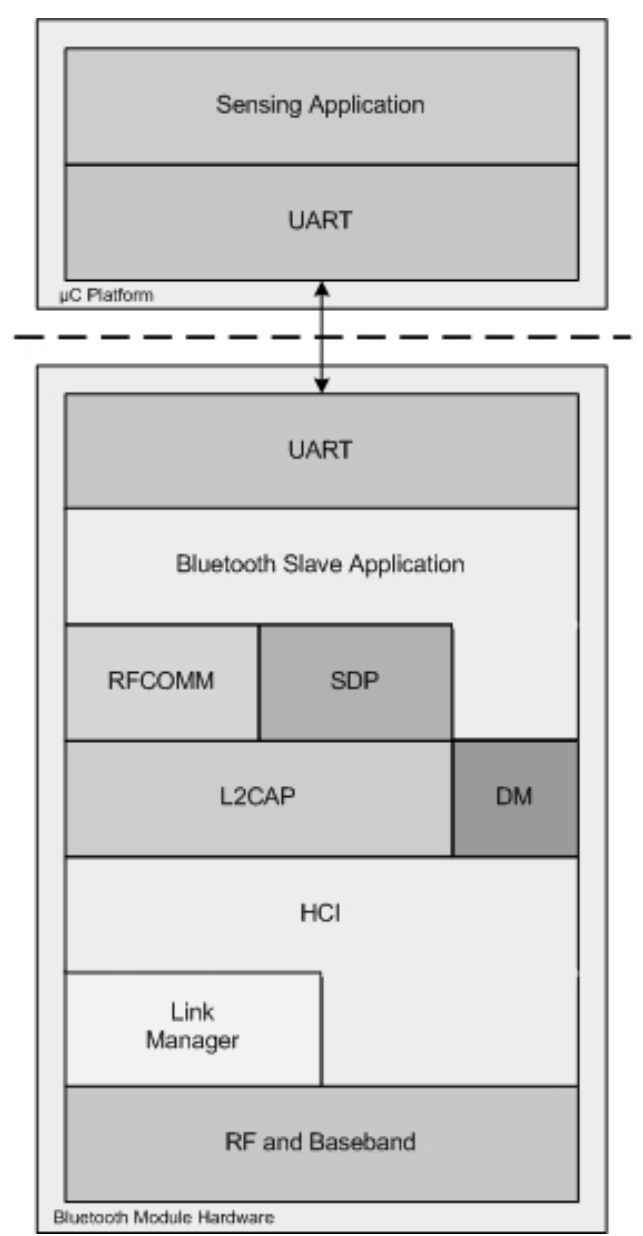

(b)

Figure 47: Bluetooth Root Node and Wireless Sensor Node Stack Architecture

(a) Single Board Computer and Bluetooth Module ( $\mathrm{HCl}$ Firmware) and (b) Sensor Platform and Bluetooth Module (RFCOMM Firmware)

\section{Test Hardware Limitations}

There are several limitations in using the described hardware. The WinCE implementation of the Bluetooth stack only supports a single Bluetooth radio [66]. This means that the Multiple Piconet Network Structure (described in 3.2.5 Multiple Piconet Bluetooth Network Structure) will require the attached Bluetooth chipsets to be flashed with the full version of the stack. This will result in a reduced maximum throughput, due to the reduced resources of the Bluetooth chipset. This limitation is further discussed in chapter 5.2.4 Multiple Piconet Bluetooth Network Structure Testing. The Intrinsic SBC UART drivers were precompiled with a maximum UART baud rate of $115.2 \mathrm{kbps}$. If this baud rate was used to communicate with the attached Bluetooth radio the maximum possible Piconet throughput would be 
approximately $90 \mathrm{kbps}$. The processor of the SBC is an Intel PXA255 with a maximum baud rate of 921.6 kbps. The precompiled UART drivers were required to be modified to increase the implemented maximum baud rate to 921.6 kbps to ensure that the theoretical Bluetooth maximum Piconet throughput was possible.

The $\mathrm{CC} \& \mathrm{C}$ modules utilise the $\mathrm{BC} 02$ chipset. The $\mathrm{HCl}$ firmware version released by $\mathrm{CSR}$ has been fully tested with the Bluetooth standard of seven active slaves [67]. However during testing it was discovered that the $\mathrm{BC} 02$ chipsets have an undocumented maximum of 10 parked slaves on a Piconet (which is not in accordance of the Bluetooth specification). This maximum number of parked slaves impacts on the Piconet Sharing Network Structure (described in 3.2.2 Piconet Sharing Bluetooth Network Structure). This limitation and its effects are further explained in 5.2.1 Piconet Sharing Bluetooth Network Structure Testing.

CSR state that the RFCOMM firmware release is optimised for point to point communications and modifications to the persistent store settings are required for point to multipoint communications. The RFCOMM firmware release notes state that CSR have only tested an RFCOMM link between a master device and up to three slaves [29]. This node limitation is due to the reduced resources of the onboard processor on the BC02 compared with the Intel PXA255 processor on the Intrinsic SBC. The RFCOMM firmware release notes also quote a maximum RFCOMM throughput of approximately $380 \mathrm{kbps}$ [29].

CSR released a document describing the Scatternet support of the $\mathrm{HCl}$ firmware release [68]. This document describes what a Scatternet is and explains which forms of Scatternet are possible with the BC02. CSR describe a master / slave Scatternet mentioning that the BC02 has limited support for this structure. There is no mention as to node or throughput limitations imposed by this network structure. There has been no documentation released that describes Scatternet support for the RFCOMM firmware release which has reduced available resources. Hardware testing is required to verify if Scatternet capability is possible with the RFCOMM firmware release.

The Bluetooth Wireless Sensor Network Testing section of this document is comprised of two parts; hardware testing standard Bluetooth piconets to examine the test hardware's capabilities and hardware implementation and testing of the developed Bluetooth network structures.

\subsection{Standard Bluetooth Piconet Testing}

Preliminary testing was done on the available Bluetooth hardware to examine the limitations in the test Bluetooth hardware and software implementations. Other tests that were conducted measured important parameters of the Bluetooth modules such as range, unparking times and power usage. The results from these tests are important when choosing which Bluetooth network structure is appropriate in different wireless sensor applications. These results also effect the implementation of the chosen network -84 - 
structure. After this point to point Bluetooth testing was complete standard Bluetooth piconet structures were established using the single board computer based root node as a master and also a Bluetooth module with integrated stack layers. Throughput results were measured with both types of Piconet master devices.

\subsubsection{Bluetooth Wireless Sensor Node Testing}

The CC\&C Bluetooth modules need to be tested to ensure that they are suitable for the wireless sensor application. The first test that was undertaken was to measure the entire modules power usage in different conditions and Bluetooth power modes. This allowed the length of operation to be estimated for different battery types. The connection and reconnection time of the wireless node is important as the devices will need to connect and reconnect to each of the root nodes in the wireless sensor network. The unparking times of the wireless sensor nodes affects the performance of several of the proposed network topologies. Measuring these parameters over a series of tests allows a clearer picture of the test hardware and softwares capabilities.

\section{Power Consumption}

Table 15 and Table 16 are the results of the power usage tests on the Bluetooth modules. These tests were using a supply voltage of 3.3 volts and the UART baud rate was set at $115.2 \mathrm{kbps}$. The measured power usage of the Bluetooth modules (along with the sensor platform) will determine how long an athlete can be continually monitored (in sport monitoring applications) for a given power supply.

\begin{tabular}{|l|l|l|}
\hline Module State & $\begin{array}{l}\text { Master Current } \\
\text { Drawn } \\
\text { (VCC = 3.3 V) }\end{array}$ & $\begin{array}{l}\text { Slave Current } \\
\text { Drawn } \\
\text { (VCC = 3.3 V) }\end{array}$ \\
\hline Idle & $2.25 \mathrm{~mA}$ & $2.25 \mathrm{~mA}$ \\
\hline LED constantly on & $3.85 \mathrm{~mA}$ & $3.85 \mathrm{~mA}$ \\
\hline LED blinking at 10Hz & $5.25 \mathrm{~mA}$ & $5.25 \mathrm{~mA}$ \\
\hline Inquiry & $67.45 \mathrm{~mA}$ & \\
\hline Inquiry Scan & & $4 \mathrm{~mA}$ \\
\hline Page & $69.3 \mathrm{~mA}$ & \\
\hline Page Scan & & $3.5 \mathrm{~mA}$ \\
\hline Inquiry Scan and Page Scan & & $6.55 \mathrm{~mA}$ \\
\hline Active (full data) (Constant LED) & $38.3 \mathrm{~mA}$ & $29.95 \mathrm{~mA}$ \\
\hline Active (no data) (Constant LED) & $8.9 \mathrm{~mA}$ & $32.7 \mathrm{~mA}$ \\
\hline
\end{tabular}

Table 15: Bluetooth Module Power Usage

$$
-85-
$$




\begin{tabular}{|l|l|}
\hline Low Power Connection States & $\begin{array}{l}\text { Slave Current } \\
\text { Drawn } \\
\text { (VCC = 3.3 v) }\end{array}$ \\
\hline Active (full data) (Constant LED) & $29.95 \mathrm{~mA}$ \\
\hline Active (no data) (Constant LED) & $32.7 \mathrm{~mA}$ \\
\hline Active (no data) (no LED) & $31.15 \mathrm{~mA}$ \\
\hline Park (no LED) & $2.4 \mathrm{~mA}$ \\
\hline Park (Blinking LED at 10 Hz) & $5.6 \mathrm{~mA}$ \\
\hline Hold (no LED) & $3.95 \mathrm{~mA}$ \\
\hline Hold (Blinking LED at 10 Hz) & $5.2 \mathrm{~mA}$ \\
\hline Sniff (sniff length = 1.28s) (no LED) & $2.35 \mathrm{~mA}$ \\
\hline Sniff (sniff length = 1.28s) (Blinking LED at 10 Hz) & $5.45 \mathrm{~mA}$ \\
\hline Sniff (sniff length = 0.04s) (no LED) & $5 \mathrm{~mA}$ \\
\hline Sniff (sniff length = 0.04s) (Blinking LED at 10 Hz) & $7.5 \mathrm{~mA}$ \\
\hline Table 16: Bluetooth Modul Powe Usage in Bluetoth & $\mathrm{L}$ ( Pow \\
\hline
\end{tabular}

Table 16: Bluetooth Module Power Usage in Bluetooth Low Power States

\section{Connection and Reconnection Latency}

Connection and reconnection latency is the time taken for an initial Bluetooth connection and subsequent reconnection. Measuring the wireless sensor nodes connection and reconnection latencies is important for estimating how long the wireless links will take to recover after a temporary disconnection or root node hand off procedure. The initial connection latency to a previously unconnected node will be much larger than if the device is being reconnected to (assuming that no inquiry procedure has been performed).

When a Bluetooth device performs an Inquiry to determine the devices in range it collects a list of the devices BD_ADDRs and also the current clock offsets of the devices. The Inquiring device can then uses this information to approximately calculate where the Bluetooth device is currently in the Bluetooth hopping pattern and this will greatly reduce the connection time. As the Bluetooth version 1.1 Inquiry procedure has been deemed impractical for use in the developed wireless sensor networks we must examine how long it will take to connect to a Bluetooth device with out the clock phase information. If a device is reconnecting to the same device it will have knowledge of the other devices clock offset and this will greatly reduce the connection time (as with connecting after an Inquiry procedure). As the Bluetooth structures will be connecting and reconnecting to wireless sensor nodes due to temporary drop outs and handoff situations the connection and reconnection times of the utilised Bluetooth hardware must be measured. Connection and reconnection was tested between different nodes multiple times to determine 
the average connection and reconnection delay and the standard deviation of the delay times. Figure 48 and Table 17 summarise the Bluetooth connection and reconnection time testing.

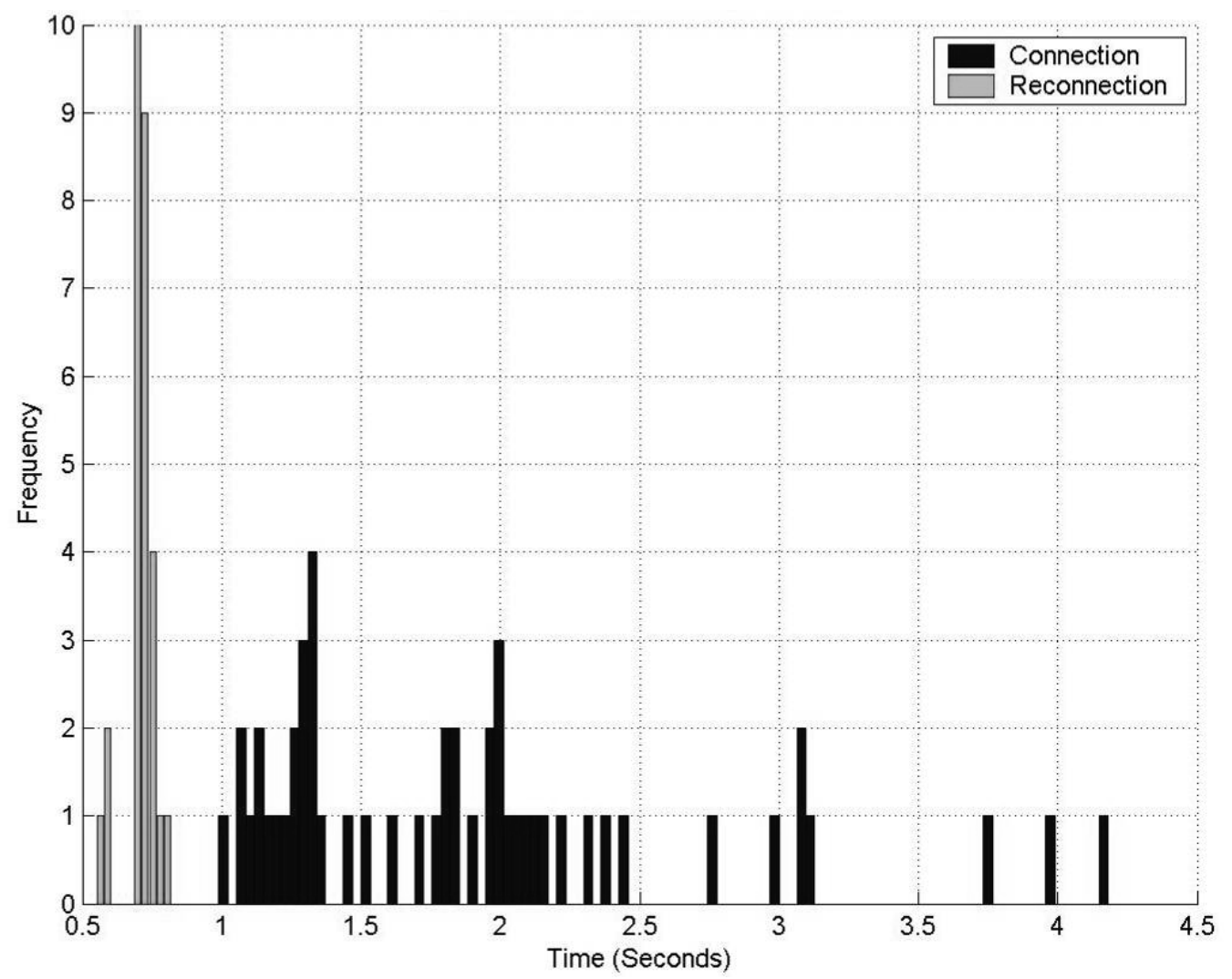

Figure 48: Bluetooth Device Connection and Reconnection Testing

\begin{tabular}{|l|l|l|}
\hline Connection Type & \multicolumn{1}{l|}{$\begin{array}{l}\text { Mean } \\
\text { (Seconds) }\end{array}$} & $\begin{array}{l}\text { Standard } \\
\text { Deviation } \\
\text { (Seconds) }\end{array}$ \\
\hline Initial Connection Time & 1.9035 & 0.7082 \\
\hline Reconnection Time & 0.7696 & 0.0540 \\
\hline
\end{tabular}

Table 17: Average Connection and Reconnection Times

As described by the Bluetooth specification the time taken to reconnect to a Bluetooth device is quicker than an initial connection (without Inquiry procedure). This is due to the connecting device having to use a random value for the far devices hopping phase offset. This difference in connection latency highlights why passing the Bluetooth address and hopping phase offset of the wireless sensor nodes between root nodes is an important part of the proposed hand off procedure. 


\section{Unparking Devices Latency}

The time taken to unpark a device on the Piconet has a measurable effect on the throughput of the proposed Piconet Sharing Network Structure (described in Chapter 3.2.2 Piconet Sharing Bluetooth Network Structure). This is due to the longer the time taken to unpark the slave device, the longer the device remains inactive on the Piconet and cannot transmit the wireless sensor data.

Once a slave on a Bluetooth Piconet is parked, it takes a discrete time to unpark the device to once again allow communications on the Bluetooth piconet. This will be affected by when the device is unparked relative to the next park beacon (as this is when a device is unparked). This means that while a device using a larger park beacon will have a longer battery life, it will generally take longer to unpark the device. Since the Piconet Sharing Network Structure (outlined in 3.2.2 Piconet Sharing Bluetooth Network Structure) continually parks and unparks the connected nodes, it is important to measure the time it takes to unpark a device. Figure 49 and Table 18 shows the measured unparking times for the Bluetooth hardware modules.
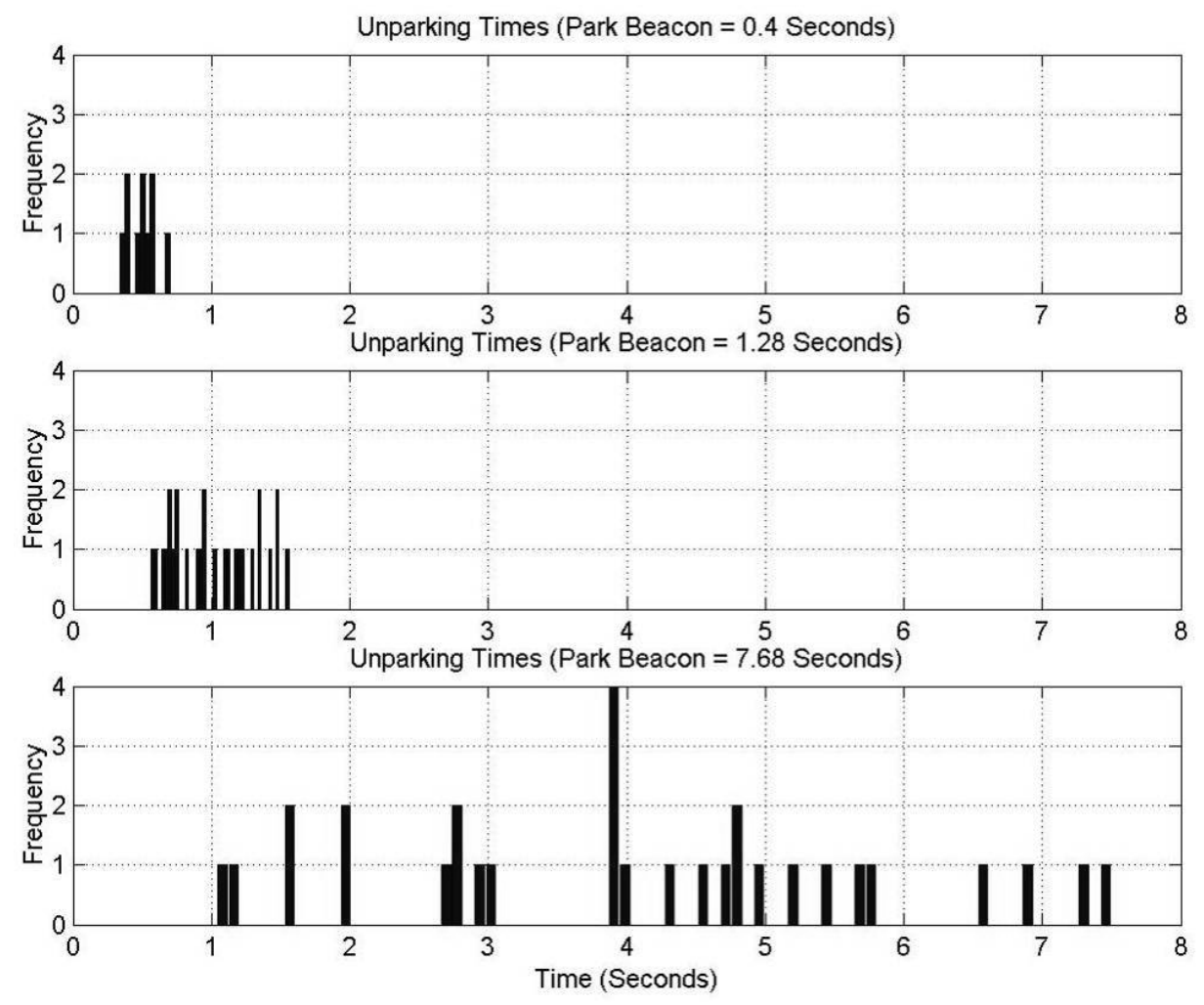

Figure 49: Bluetooth Unparking Times for Varying Park Beacon Lengths 


\begin{tabular}{|l|l|l|}
\hline $\begin{array}{l}\text { Park Beacon Interval } \\
\text { (Seconds) }\end{array}$ & $\begin{array}{l}\text { Mean } \\
\text { (Seconds) }\end{array}$ & $\begin{array}{l}\text { Standard Deviation } \\
\text { (Seconds) }\end{array}$ \\
\hline 0.4 & 0.4970 & 0.7082 \\
\hline 1.28 & 1.0170 & 0.3048 \\
\hline 7.68 & 4.0510 & 1.8032 \\
\hline
\end{tabular}

Table 18: Average Unparking Times for Varying Park Beacon Lengths

As per the Bluetooth specification the longer the park beacon interval the longer the average delay in unparking a device. A trade-off between battery life and unparking latency must be made when selecting the park beacon interval.

\section{Communication Range}

The Bluetooth hardware must have a suitable range for the wireless sensor application. As the range increases from the root node the throughput decreases as the signal to noise ratio decreases. The effect of the communication range had to be considered when implementing the proposed wireless sensor network structures.

The Bluetooth module is a class 1 device meaning that it has a maximum power output of $100 \mathrm{~mW}$ (20 $\mathrm{dBm})$ and an approximate range of 100 meters. If the application does not require a 100 meter range then the output power can be reduced and the devices battery life can be extended. The device range was measured by establishing an RFCOMM connection between two devices and measuring the throughput drop off as the range increases. The baud rate of the Bluetooth hardware was set to 115.2 kbps and the testing device creating the data was set to create data at approximately $80 \mathrm{kbps}$. Figure 50 shows the measured relationship between communication range and throughput. 


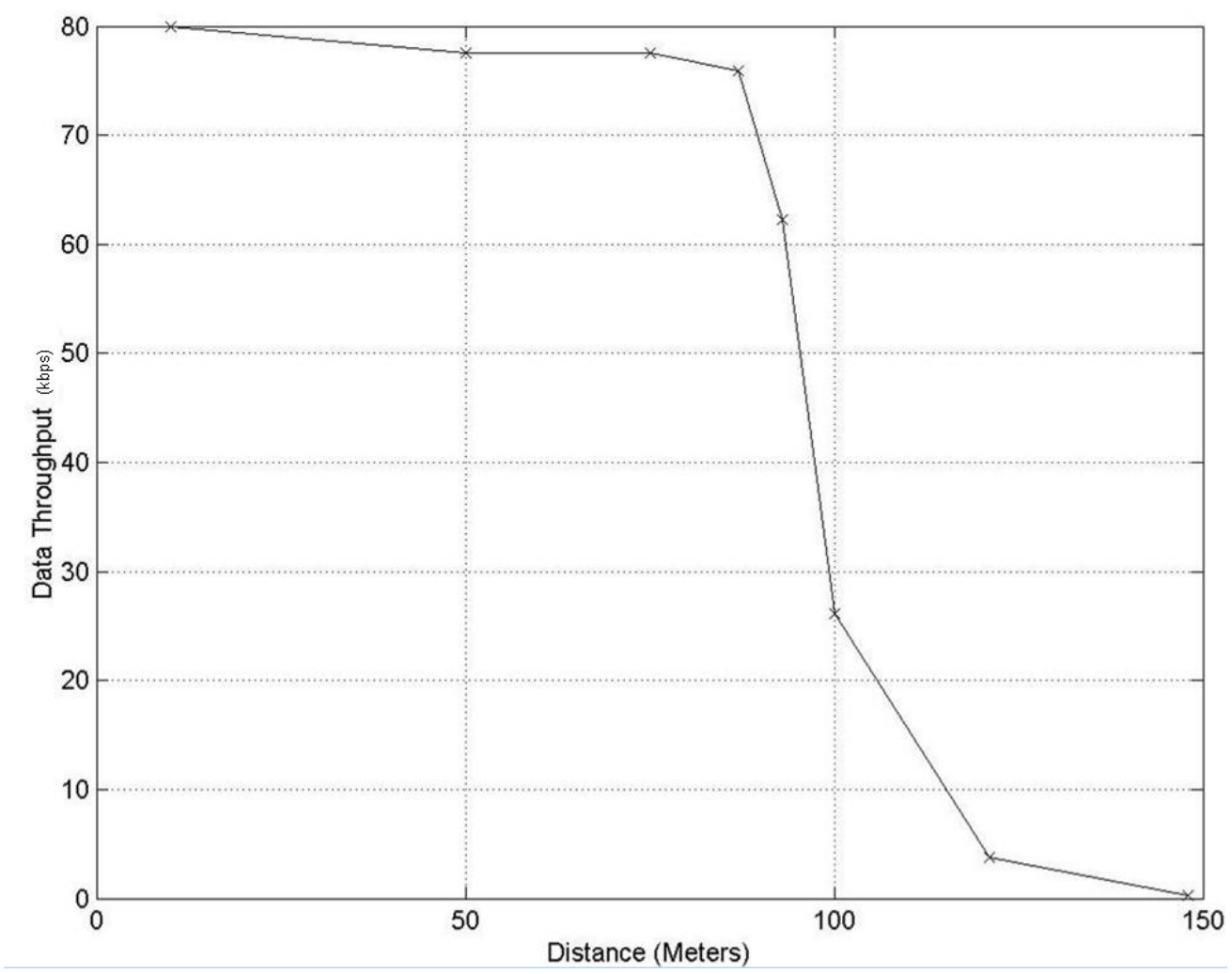

Figure 50: Throughput Drop Off with Increases Communication Range

As the communication range increases the bit error rate (BER) of the link increases due to the received power dropping. At approximately 95 meters the throughput had dropped by $50 \%$ due to packet errors received. Communications between the devices was maintained past 200 meters at low throughput rates. These tests were conducted with the near omidirectional antennas supplied as part of the Bluetooth modules. If directional antennas were used then the communication range of the device would be much larger, due to the high gain of the directional antennas.

\subsubsection{Root Node Created Standard Piconet Bluetooth Network Structure Testing}

All of the proposed network structures utilise the single board computer as the root node and the Bluetooth modules as the wireless sensor nodes. A standard Piconet using this configuration was implemented to compare the performance of the test hardware with the Bluetooth Specification.

Using the single board computer (upper stack layers) and Bluetooth module (lower stack layers) as the Piconet master node point to multipoint based connections were made to a number of slave nodes. As the single board computer forms the root node for all of the Bluetooth network structures it is important to verify that no unexpected throughput constraints exist that will limit the Bluetooth network throughput. Standard Windows sockets were used to establish RFCOMM based connections between the WinCE 
based root node and the BlueStack based wireless sensor nodes. The corresponding BlueStack RFCOMM messages can be found in Appendix E: Bluetooth RFCOMM Message Sequence Chart. The measured throughput per slave for a root node master can be seen in Figure 51.

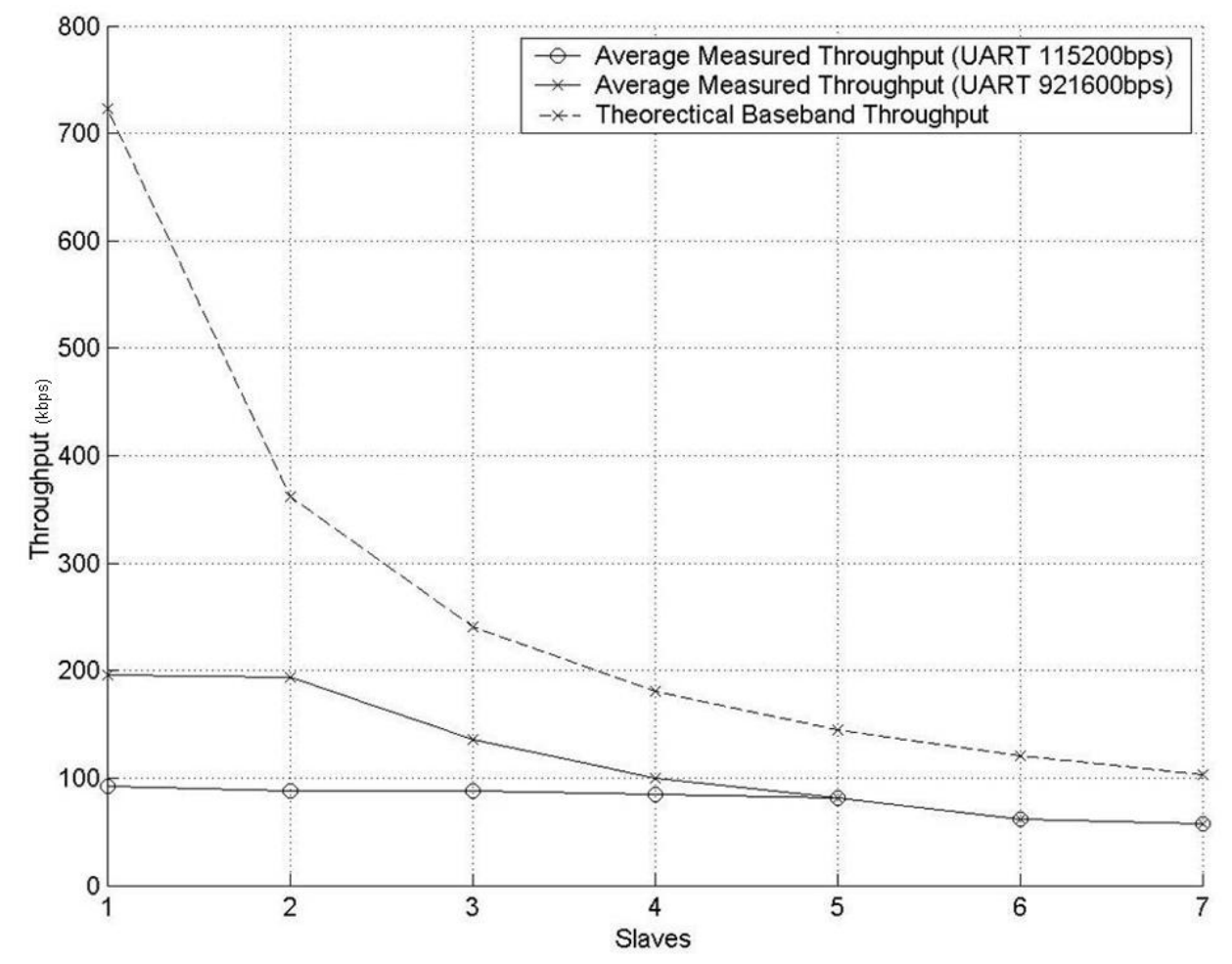

Figure 51: Measured Throughput per Slave for Root Node Created Standard Piconet

During root node Piconet testing it was discovered that the precompiled UART driver for the single board computer only had baud rates included up to 115.2 bps (the Intel processor supports UART baud rates up to $921.6 \mathrm{kbps}$ ). Figure 51 clearly shows the effect of the default UART setting by the maximum throughput being limited to approximately $92.160 \mathrm{kbps}$ (the maximum throughput of a UART baud rate of 115.2 bps).Modifying the precompiled baud rate table allowed the UART baud rate to be increased to the maximum $921.6 \mathrm{kbps}$ which allows for the maximum Bluetooth piconet throughput. The piconet throughput at this baud rate was limited to approximately $200 \mathrm{kbps}$. This is the maximum measured throughput of the RFCOMM link between the WinCE device and the CSR based Bluetooth modules. Figure 51 also shows the theoretical baseband throughput for the Bluetooth piconet for comparison. The drop in throughput between the measured RFCOMM piconet throughput (with $921.6 \mathrm{kbps}$ baud rate) and the theoretical baseband throughput is a combination of RFCOMM overhead, stack implementation limitations and non optimum connection settings. 


\subsubsection{Mobile Node Created Standard Piconet Bluetooth Network Structure Testing}

Many of the proposed network structures all use the Bluetooth modules as masters of Bluetooth Piconets. It was therefore necessary to test the throughput of standard Bluetooth Piconet with a CSR based Bluetooth module as the Piconet master.

In a firmware release note CSR state that an RFCOMM link has only been tested between a master device and three slaves [29]. Hardware testing showed that a Piconet with more than 4 slaves actively communicating data caused the Piconet master to stop functioning. The measured RFCOMM throughput for a standard Bluetooth Piconet created with a Bluetooth module appears below as Figure 52.

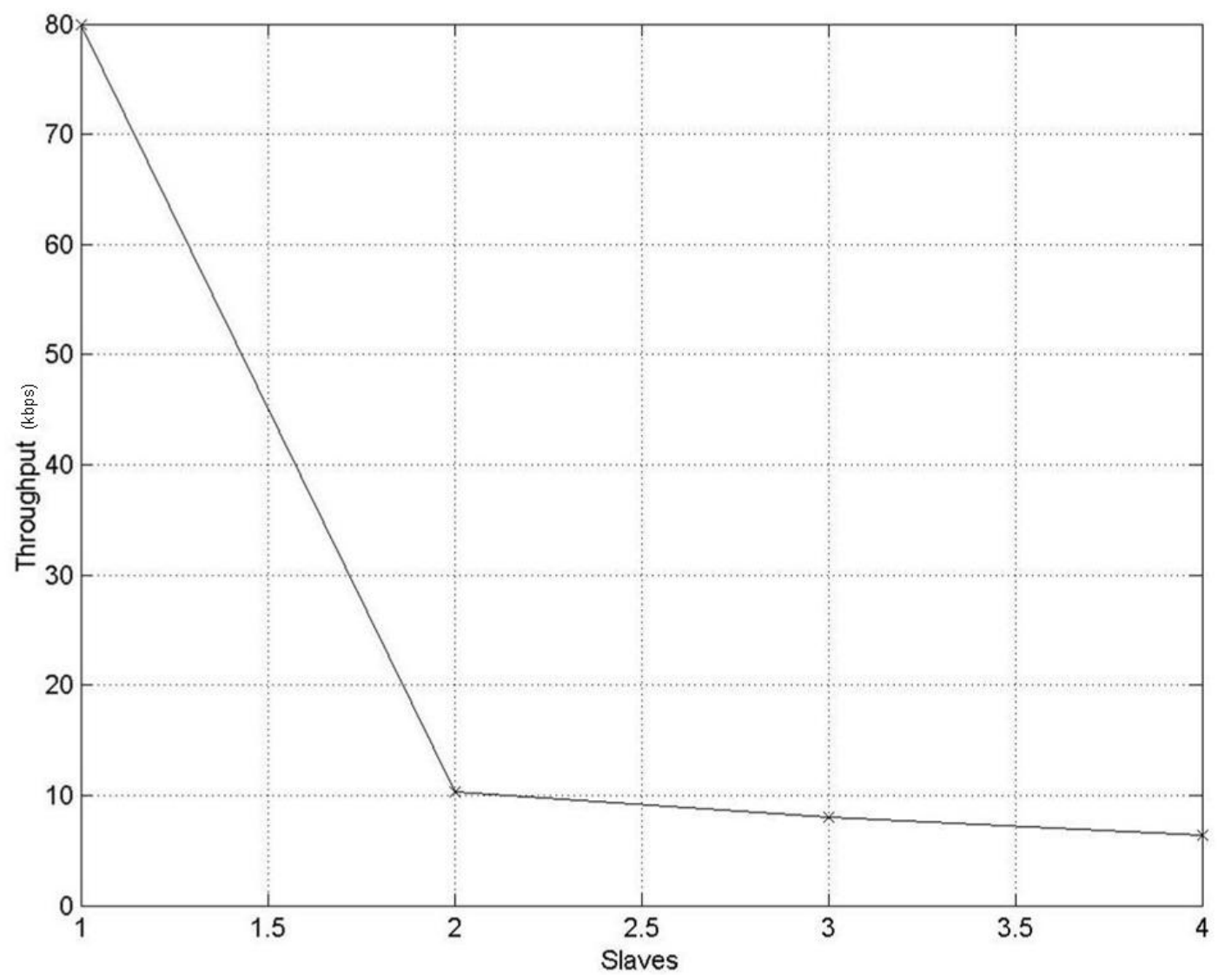

Figure 52: Measured Throughput per Slave for Bluetooth Module Created Standard Piconet

The above testing was completed with a module UART baud rate of $115.2 \mathrm{bps}$. Therefore the maximum piconet throughput was limited to approximately $90 \mathrm{kbps}$. The test data generation devices used generated data at an approximate maximum of $80 \mathrm{kbps}$. 


\subsection{Developed Bluetooth Network Structure Testing}

With the basic hardware functionality assessed, investigation into the performance of the proposed Bluetooth wireless sensor network structures was undertaken. Using the Intrinsic Cerfboard single board computer (SBC) and CC\&C Technologies Bluetooth modules the Bluetooth network structures described in Chapter 3.2 Bluetooth Wireless Sensor Network Structures was implemented and tested. Throughput per node results were measured for each of the network structures to determine if each satisfied the requirements for the targeted wireless sensor network application.

Each network structure uses a Bluetooth root node which acts as a gateway between the Bluetooth wireless sensor network and the high speed backbone to the central data server. This Bluetooth root node is built from a Cerfboard SBC (running WinCE) and a CC\&C Technologies Bluetooth module (running BlueStack). The WinCE creates Bluetooth RFCOMM connections to the wireless sensor nodes using standard Windows Sockets. The Windows Socket interface to the Bluetooth stack also allows for devices to be placed into the Bluetooth low power states (sniff, hold and park mode). The wireless sensor nodes are using the CSR BlueStack Bluetooth stack. The corresponding stack messages to each network structure have been placed into appendices and referenced where appropriate.

Testing was done with a limited number of sensor nodes and serval assumptions have been made to extrapolate the measured results into the full network structure results. Where result extrapolation has occurred the assumptions have been clearly stated and explained. Each structure is presented with both measured results and then extrapolated results for the total possible number of network nodes. The wireless sensor application that the network has been designed for requires a total throughput of 4500 bps (three channels of 10 bit data at 150 times a second). Each network structure has been compared to this throughput requirement. A summary of each network structure's results is then presented and comments were made regarding the appropriateness of each structure for various wireless sensor networks applications.

\subsubsection{Piconet Sharing Bluetooth Network Structure Testing}

The Piconet Sharing network structure allows more than seven Bluetooth slaves to communicate on a Piconet by sharing the Piconet active member addresses (AM_ADDR) between the slaves on the Piconet. This effectively increases the time division communications on the Piconet beyond the maximum of seven active slaves. Chapter 3.2.2 Piconet Sharing Bluetooth Network Structure outlines the network structure in detail. Appendix $\mathrm{F}$ contains the corresponding BlueStack message sequences for parking and Unparking Bluetooth devices. 
Preliminary testing of the Bluetooth hardware found an undocumented limitation in the CSR BlueCore Chipset. It was discovered that a maximum of 10 devices can be parked at a time. This is not in accordance with the Bluetooth specification. This limits the number of devices that can be implemented in the piconet sharing structure. Testing was still undertaken with the CSR Chipset based CC\&C modules, however assumptions had to be made to extrapolate the testing results to beyond the maximum parked device limit. To achieve extrapolated results for the Piconet Sharing network structure while keeping the number of parked device below the CSR parked slaves limit, only the first Piconet Sharing Group of each test was fully populated. This means that group 0 of each test had the maximum number of slaves per group for each test while each other piconet sharing group only contained a reduced number of slaves. Figure 53 shows an example of the Piconet Sharing node extrapolation.

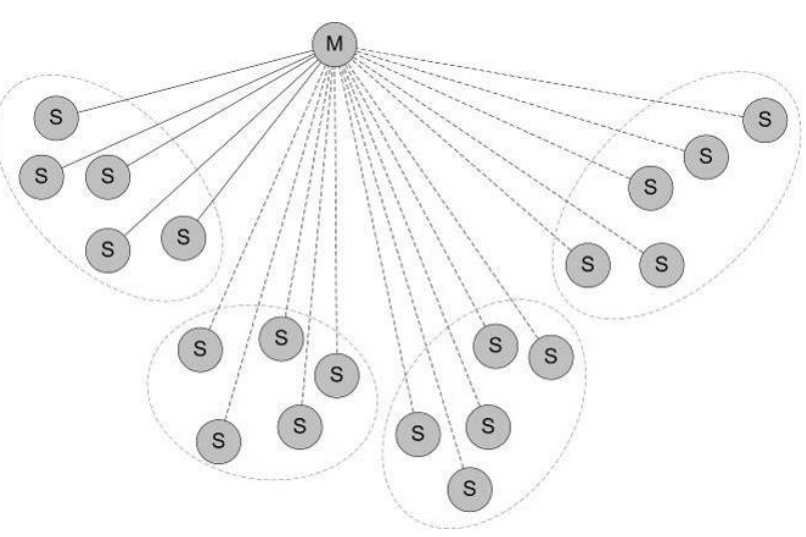

(a)

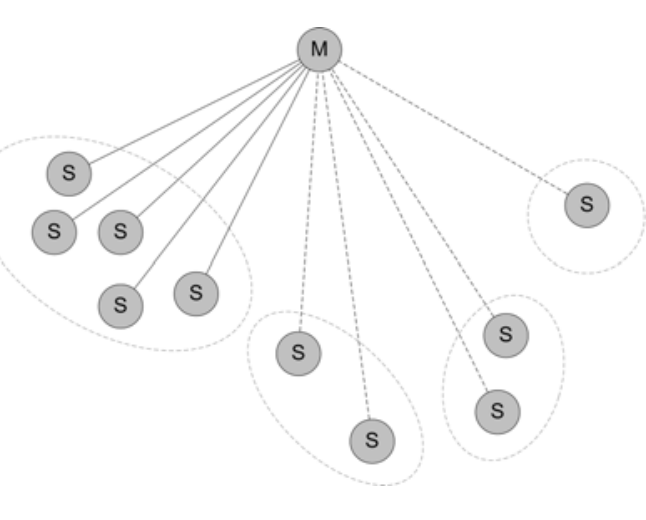

(b)

Figure 53: Piconet Sharing Node Extrapolation

(a) Extrapolated Piconet Sharing Structure and (b) Actual Tested Piconet Sharing Structure

The full set of Piconet Sharing testing results has been included as Appendix G: Piconet Sharing Testing Results. Using these testing results and the method of extrapolation show in Figure 53 the extrapolated Piconet Sharing results were calculated for up to 20 nodes. Table 19, Table 20 and Table 21 show the extrapolated Piconet Sharing results. These testing results were taken test data sources running with an UART baud rate of 115.2 bps. This has limited the throughput results of the Piconet Sharing Network Structure as the full available network bandwidth was not utilised in testing. Retesting the Piconet Sharing Structure with data sources running at a higher baud rate will yield higher throughput results. 


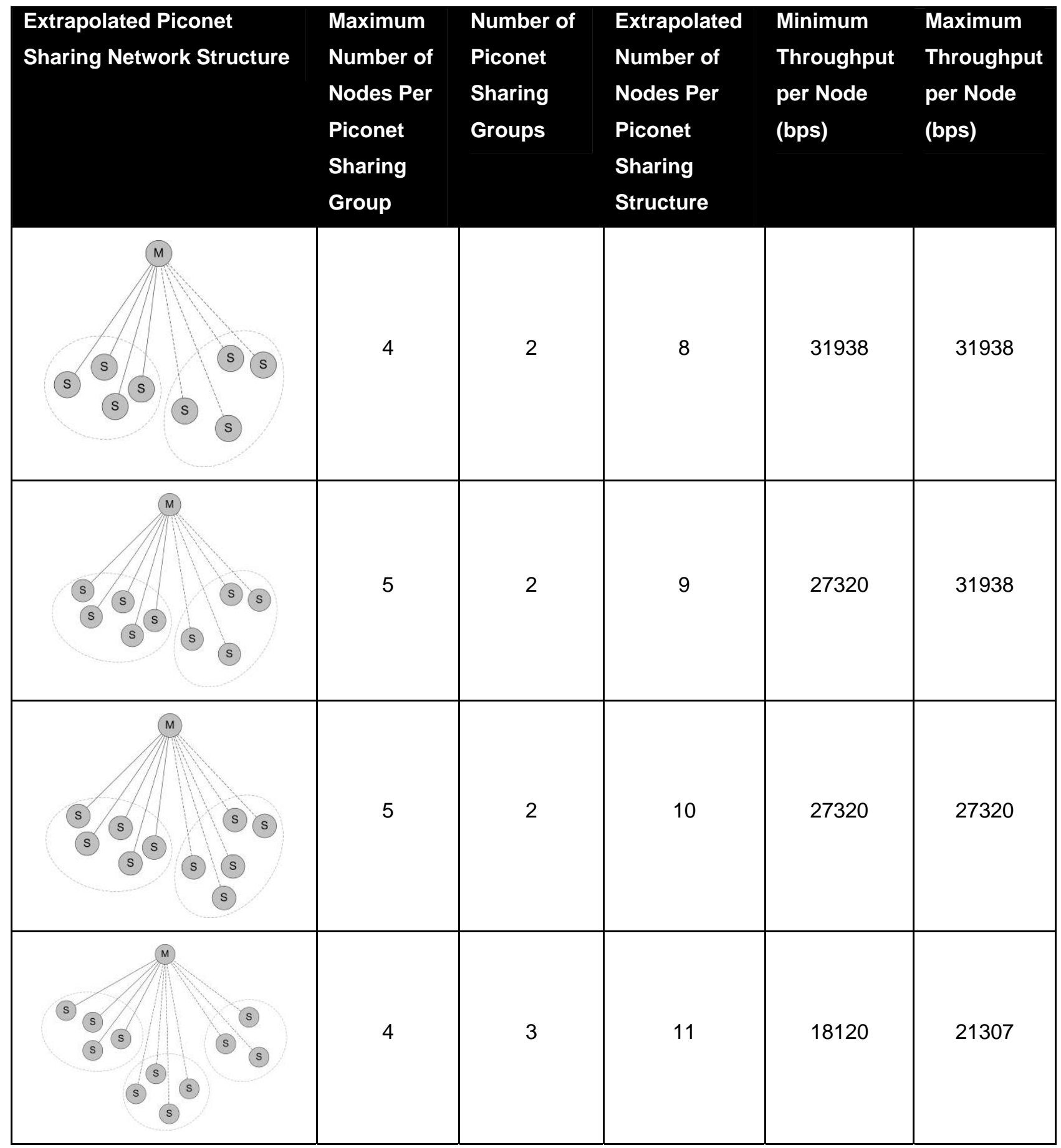

Table 19: Extrapolated Piconet Sharing Hardware Testing Results

$-95-$ 


\begin{tabular}{|c|c|c|c|c|c|}
\hline $\begin{array}{l}\text { Extrapolated Piconet } \\
\text { Sharing Network Structure }\end{array}$ & $\begin{array}{l}\text { Maximum } \\
\text { Number of } \\
\text { Nodes Per } \\
\text { Piconet } \\
\text { Sharing } \\
\text { Group }\end{array}$ & $\begin{array}{l}\text { Number of } \\
\text { Piconet } \\
\text { Sharing } \\
\text { Groups }\end{array}$ & $\begin{array}{l}\text { Extrapolated } \\
\text { Number of } \\
\text { Nodes Per } \\
\text { Piconet } \\
\text { Sharing } \\
\text { Structure }\end{array}$ & $\begin{array}{l}\text { Minimum } \\
\text { Throughput } \\
\text { per Node } \\
\text { (bps) }\end{array}$ & $\begin{array}{l}\text { Maximum } \\
\text { Throughput } \\
\text { per Node } \\
\text { (bps) }\end{array}$ \\
\hline 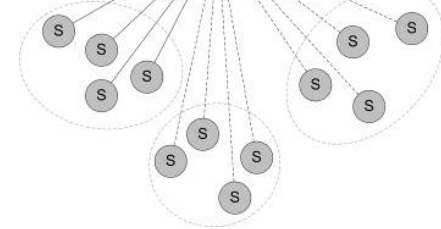 & 4 & 3 & 12 & 18120 & 18120 \\
\hline 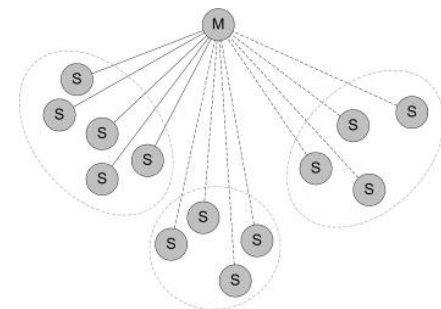 & 5 & 3 & 13 & 17672 & 18120 \\
\hline 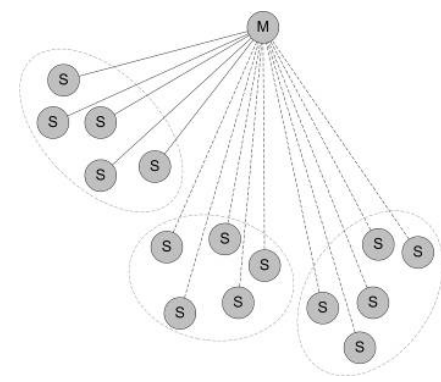 & 5 & 3 & 15 & 17672 & 17672 \\
\hline 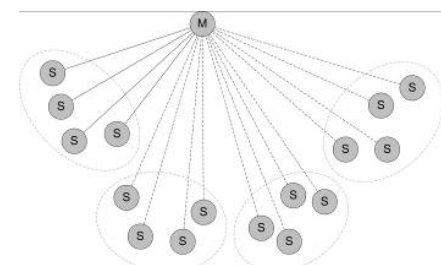 & 4 & 4 & 16 & 14547 & 14547 \\
\hline
\end{tabular}

Table 20: Extrapolated Piconet Sharing Hardware Testing Results 


\begin{tabular}{|c|c|c|c|c|c|}
\hline $\begin{array}{l}\text { Extrapolated Piconet } \\
\text { Sharing Network Structure }\end{array}$ & $\begin{array}{l}\text { Maximum } \\
\text { Number of } \\
\text { Nodes Per } \\
\text { Piconet } \\
\text { Sharing } \\
\text { Group }\end{array}$ & $\begin{array}{l}\text { Number of } \\
\text { Piconet } \\
\text { Sharing } \\
\text { Groups }\end{array}$ & $\begin{array}{l}\text { Extrapolated } \\
\text { Number of } \\
\text { Nodes Per } \\
\text { Piconet } \\
\text { Sharing } \\
\text { Structure }\end{array}$ & $\begin{array}{l}\text { Minimum } \\
\text { Throughput } \\
\text { per Node } \\
\text { (bps) }\end{array}$ & $\begin{array}{l}\text { Maximum } \\
\text { Throughput } \\
\text { per Node } \\
\text { (bps) }\end{array}$ \\
\hline $\begin{array}{l}\text { (3) } \\
\text { (3) } \\
\text { (3) } \\
\text { (3) }\end{array}$ & 5 & 4 & 17 & 14041 & 14547 \\
\hline 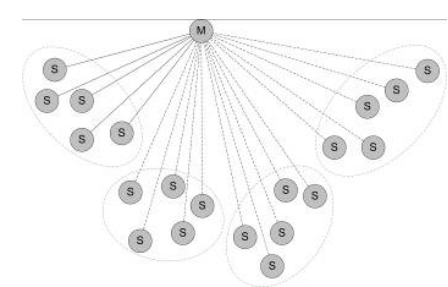 & 5 & 4 & 20 & 14041 & 14041 \\
\hline
\end{tabular}

Table 21: Extrapolated Piconet Sharing Hardware Testing Results

The throughput results outlined in Table 19, Table 20 and Table 21 were averaged over three tests running for five minutes per test. This length of time was chosen as it produced repeatable average throughput per second results. Figure 54 shows the extrapolated throughput for the Piconet Sharing Network Structure. 


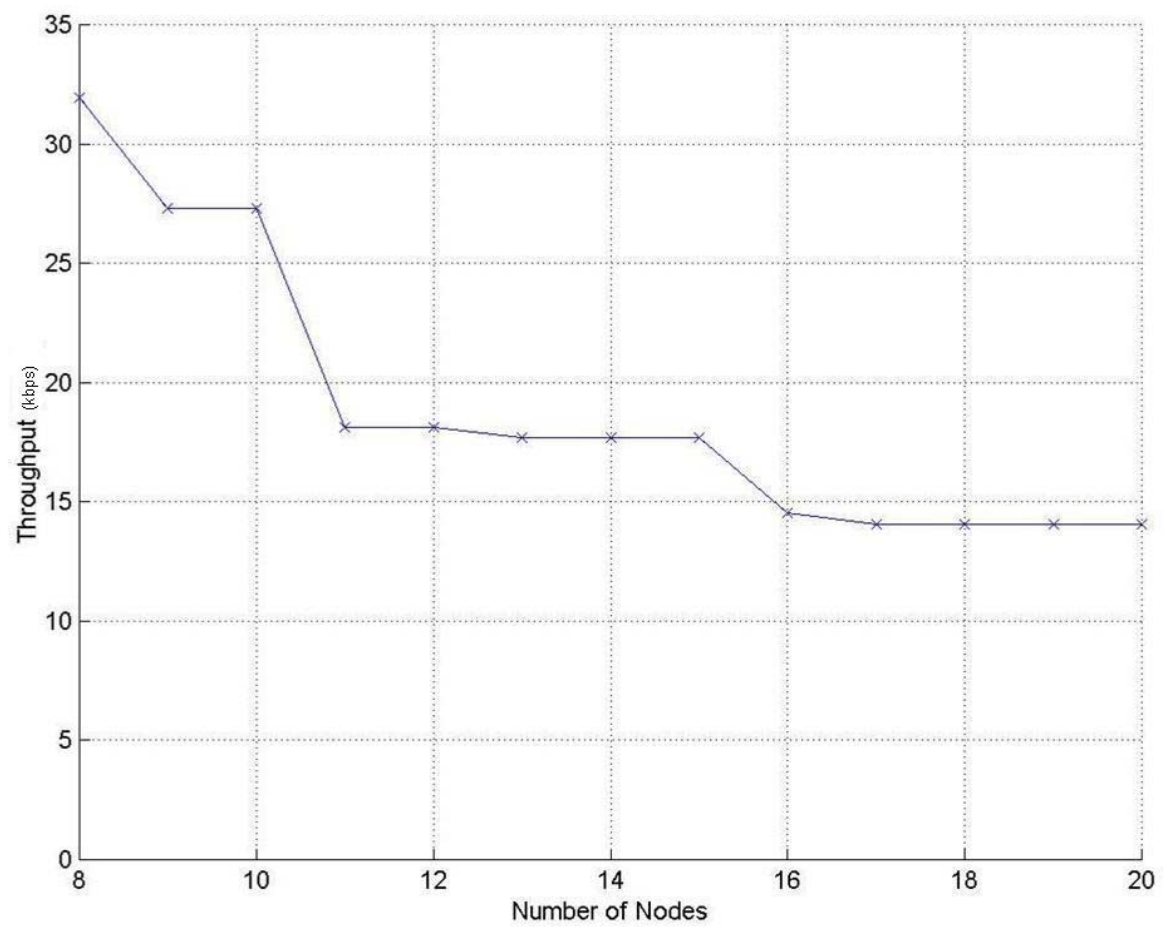

Figure 54: Extrapolated Bluetooth Piconet Sharing Network Structure Minimum Throughput

The major limitation of the Piconet Sharing Network Structure is that parked slaves can not communicate on the Piconet. This means that the longer each group remains active on the piconet the longer the device will have to wait and buffer the data until it becomes active again. During the changeover between Piconet Sharing Groups there is no communications on the Piconet. The longer the active time for each group a smaller number of group changeovers occur in a given time, increasing the average throughput. Figure 55 shows the effect on average throughput when increasing the active time of each Piconet Group on the piconet. These results were taken with a Piconet Sharing Network with three slaves per group and three Piconet Sharing groups (each slave has a UART of 115.2 bps). Figure 56 shows the latency that is inherently part of the Piconet Sharing Network Structure. 


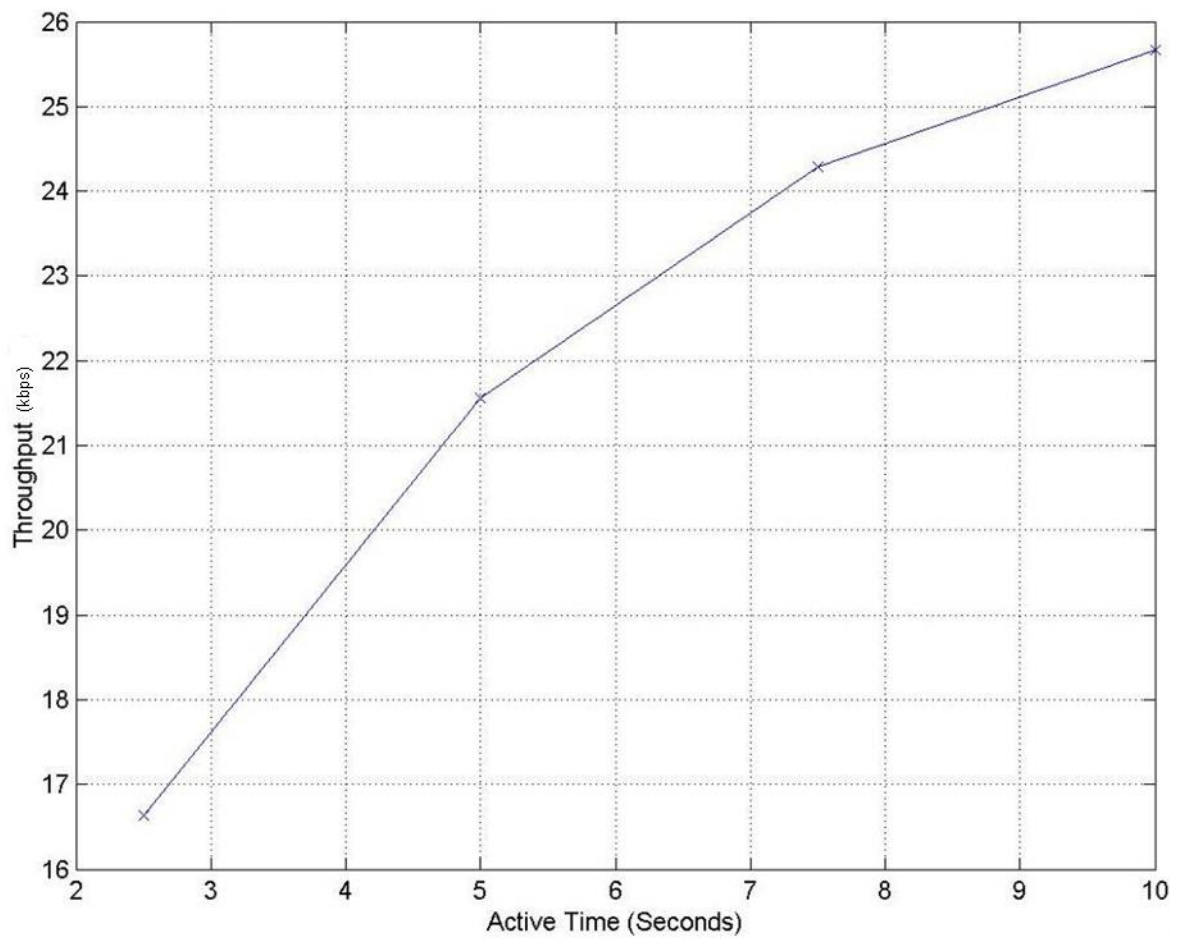

Figure 55: Effect of Average Piconet Sharing Throughput of Piconet Sharing Group Active Time

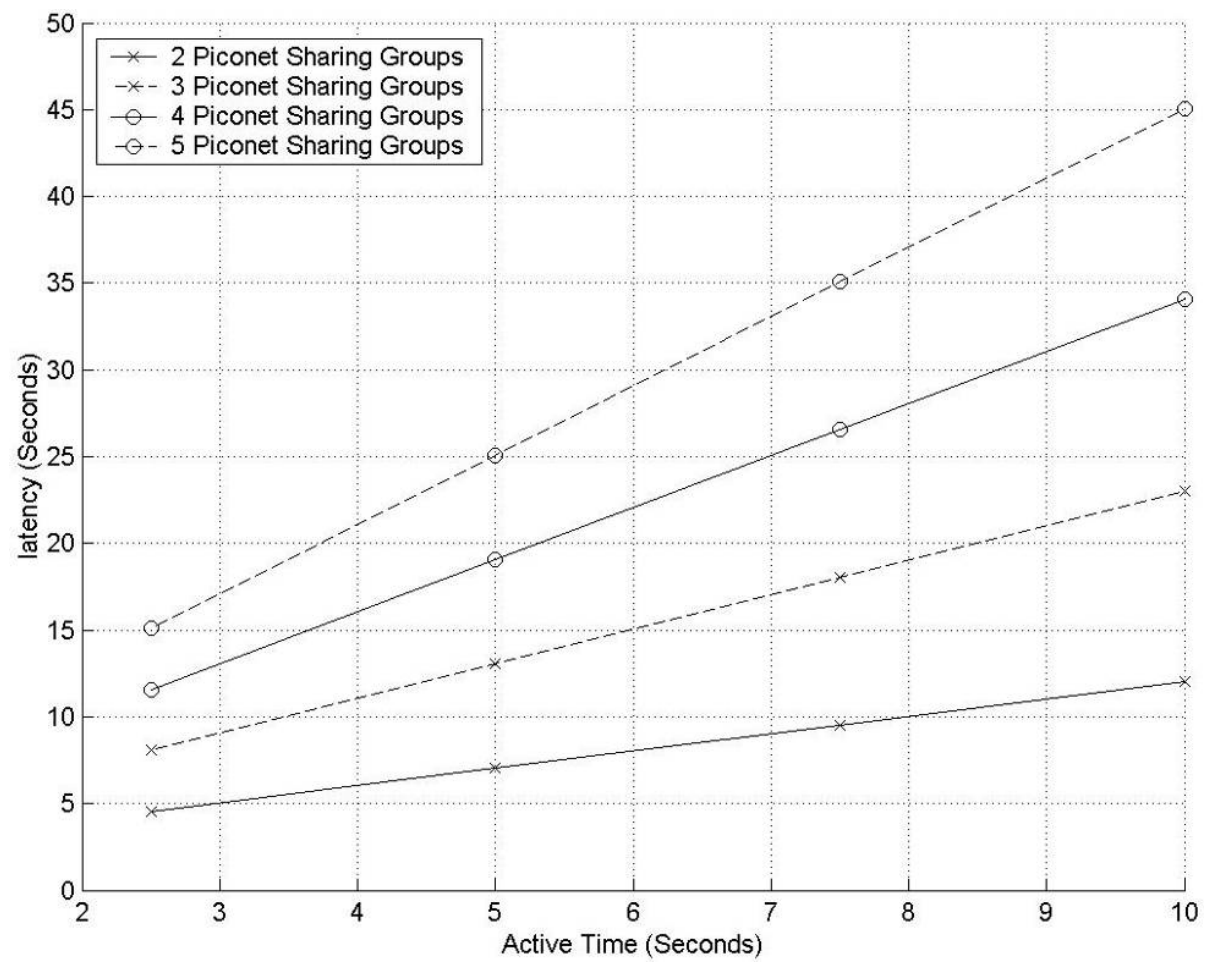

Figure 56: Piconet Sharing Network Structure Latency

-99 - 
The Piconet Sharing latency shown in Figure 56 was calculated using Equation 3. Values for average parking time and Unparking time were taken from hardware testing conducted in 5.1 Standard Bluetooth Piconet Testing.

$$
\text { Latency }=\left(T_{\text {Active }} *\left(N_{\text {Groups }}-1\right)\right)+\left(\left(T_{\text {Park }}+T_{\text {Unpark }}\right) * N_{\text {Groups }}\right) \quad \text { Equation } 3
$$

Where:

Latency = Length of time between active periods on the Piconet.

$T_{\text {Active }} \quad=$ Length of time each Piconet Group is active on the Piconet.

$N_{\text {Groups }} \quad=$ Number of Piconet Groups in the Piconet Sharing Structure.

$T_{\text {Park }} \quad=$ Length of time taken to park a device

$T_{\text {Unpark }} \quad=$ Length of time taken to unpark a device

As the latency time increases for slaves in the Piconet Sharing Structure more data is required to be buffered until the next active communication period. If the wireless sensor nodes are unable to buffer this amount of data, then data will be lost. This latency between communication periods is the major limitation of the Piconet Sharing Structure.

Piconet Sharing has been demonstrated in hardware as a technique to extend the node limitations in Bluetooth networks beyond seven devices. To be a viable option in wireless sensor networks additional Bluetooth hardware must be sourced that is able to be connected to more than ten parked devices at a time. With the current CSR BlueCore02 based hardware this network structure is not suitable for wireless sensor network applications.

\subsubsection{Scatternet Tree Bluetooth Network Structure Testing}

The Scatternet Tree Bluetooth Network Structure is formed by Bluetooth nodes acting as a master and a slave simultaneously forming a bridge between two Piconets. This allows tree structures to be created using multiple Bluetooth Piconets which allow more than seven Bluetooth devices to communicate actively at a time. Chapter 3.2.3 Scatternet Tree Bluetooth Network Structure outlines this network structure in detail. CSR have released no information regarding the RFCOMM firmware builds Scatternet capabilities. Throughput testing of this network structure demonstrated the RFCOMM firmware builds limited Scatternet capabilities.

CSR state that their $\mathrm{HCl}$ firmware build supports master / slave Scatternet formation, however give no indication to the Scatternet capabilities of the integrated RFCOMM firmware builds. The higher strain placed on the CSR BlueCore 02 Chipset by the complete Bluetooth stack may render master / slave Scatternets formation with the complete RFCOMM build impossible. The written Scatternet simulation - 100 - 
(presented in 4.2.2 Scatternet Tree Bluetooth Network Structure Simulation) allowed full control over the lower layers of the Bluetooth stack to ensure the optimum switching between piconets of the master I slave bridging nodes. The implemented hardware (BlueCore 02) and firmware (BlueStack) did not allow for such fine tuning of the lower Bluetooth baseband operations. For these reasons it is expected that the Scatternet implemented in hardware will produce lower throughput results than seen in the simulation.

The Bluetooth Scatternet firmware was written in such a matter that any mobile node can be a bridging node or just a slave node, based on instructions from the master root node. Once an RFCOMM connection has been established with a slave it immediately starts sending the buffered sensor data from the wireless sensor platform. The master can at any time request that the connected slave connect to another Bluetooth device to become a master / slave bridging node. When this occurs the master / slave bridging node sends both the attached sensor platforms data and the sensor data from the slave nodes. The bridging node must also append identification header information onto the outgoing data to identify to the master root node with wireless sensor platform the data is from. The BlueStack message sequence for the implemented master / slave Scatternet nodes can be found in Appendix $\mathrm{H}$ : Bluetooth Scatternet Message Sequence Chart.

Testing master / slave Scatternets in hardware showed that the throughput limitation was as expected the bridging nodes. Table 22 shows the hardware testing results of a single master / slave bridging node. The table only includes results with up to three slaves as beyond this the behaviour of the bridging node was unpredictable and frequently became unresponsive. This has been attributed to the limited resources of the $\mathrm{BC} 02$ Chipset. The implemented Scatternet Tree Network Structure has been limited to a maximum of two Scatternet levels. This makes the tagging of the sensor data with an identification header by the bridging node a simpler task as well as reducing the complexity of Scatternet Tree implementation. Preliminary testing of Scatternet Tree structures with more than two levels showed unpredictable results. 


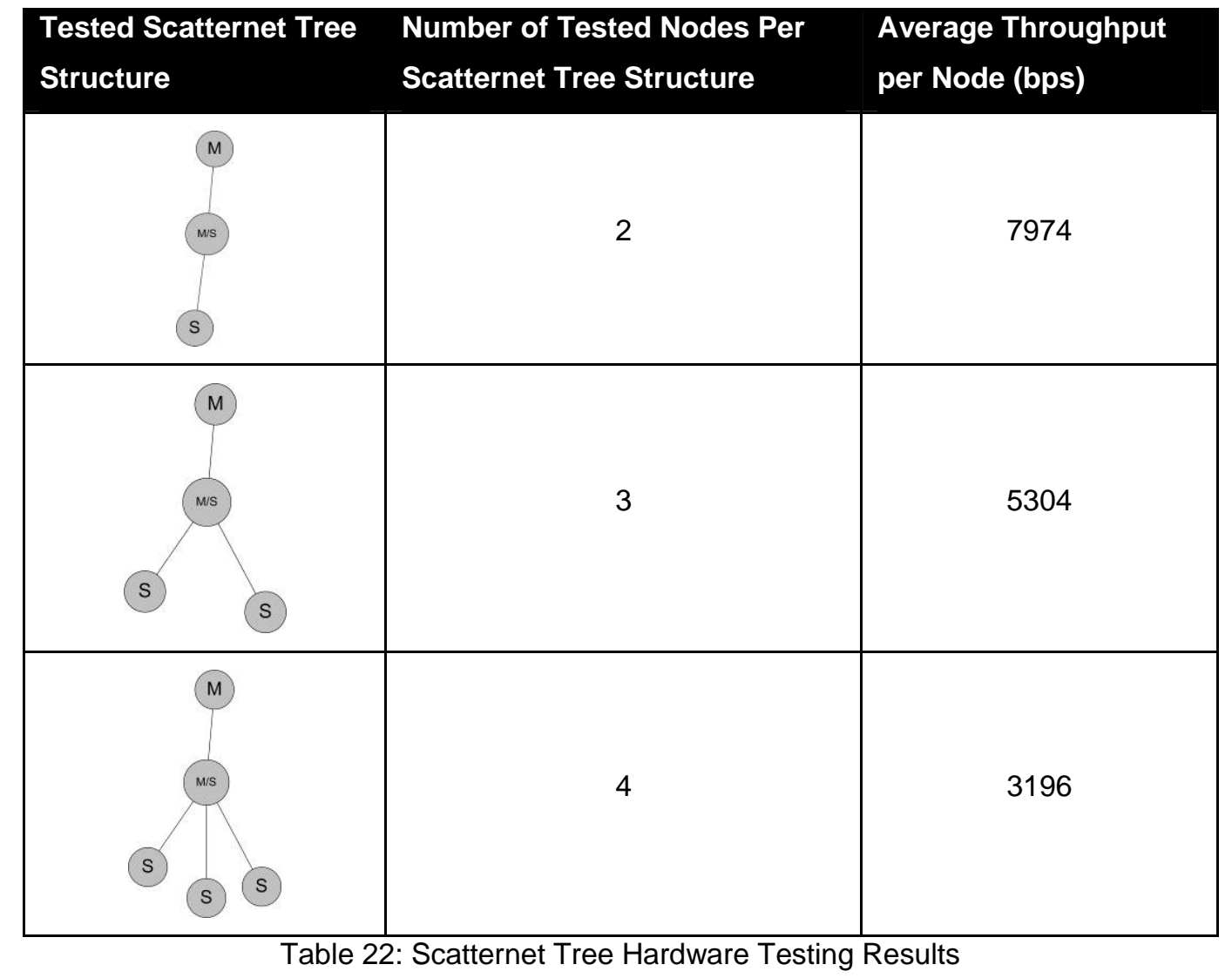

The throughput results in Table 22 have been averaged over three throughput measurement tests running for ten minutes each. This testing provided consistent average throughput per second results.

The results from the single Scatternet bridging node can be extrapolated to give the throughput results for up to 28 sensor nodes. As the major throughput of the Scatternet Network Structure is the throughput limitation of the bridging node, the lack of Piconet coexistence will have little effect on the throughput extrapolation. Table 23 summarises the extrapolated Scatternet Tree testing results. Figure 57 is the extrapolated minimum throughput for the Scatternet Tree Structure (limited to two Scatternet levels). 


\begin{tabular}{|c|c|c|c|c|}
\hline $\begin{array}{l}\text { Extrapolated Scatternet } \\
\text { Tree Network Structure }\end{array}$ & $\begin{array}{l}\text { Actual Number of } \\
\text { Tested Nodes Per } \\
\text { Scatternet Tree } \\
\text { Structure }\end{array}$ & $\begin{array}{l}\text { Extrapolated } \\
\text { Number of } \\
\text { Nodes Per } \\
\text { Scatternet Tree } \\
\text { Structure }\end{array}$ & $\begin{array}{l}\text { Minimum } \\
\text { Throughput } \\
\text { per Node } \\
\text { (bps) }\end{array}$ & $\begin{array}{l}\text { Maximum } \\
\text { Throughput } \\
\text { per Node } \\
\text { (bps) }\end{array}$ \\
\hline 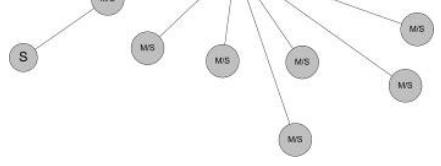 & 2 & 8 & 7974 & $\begin{array}{c}56829 * \\
\text { *(not Scatternet) }\end{array}$ \\
\hline (3) & 2 & 14 & 7974 & 7974 \\
\hline (3) & 3 & 15 & 5304 & 7974 \\
\hline 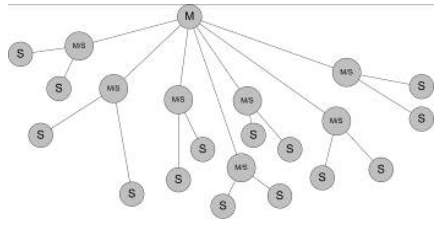 & 3 & 21 & 5304 & 5304 \\
\hline 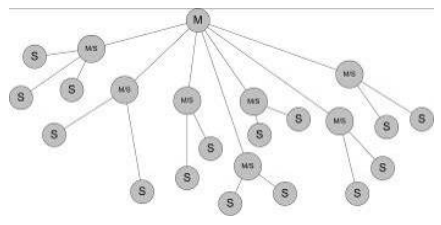 & 4 & 22 & 3196 & 5304 \\
\hline (3) (3) & 4 & 28 & 3196 & 3196 \\
\hline
\end{tabular}

Table 23: Extrapolated Scatternet Tree Hardware Testing Results 


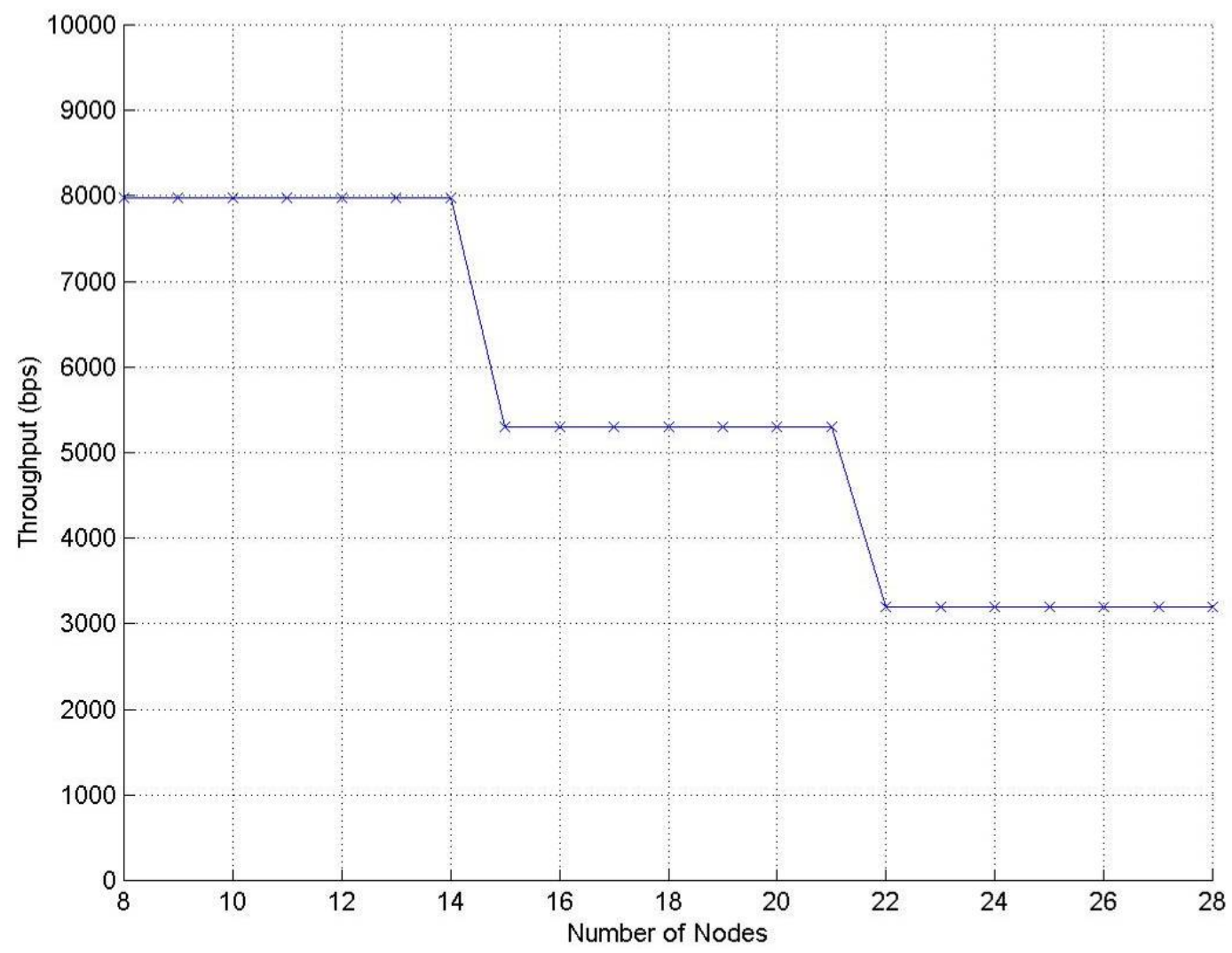

Figure 57: Extrapolated Bluetooth Scatternet Tree Network Structure Minimum Throughput

As expected the throughput results of the hardware testing were significantly lower than the simulated values. To achieve optimum Scatternet throughput results the hardware needs to be designed with Scatternet implementation in mind. Improved Scatternet results would be obtained if the upper Bluetooth layers were moved onto an additional processor freeing up resources for Scatternet implementation. Alternatively other Bluetooth hardware needs to be sourced that provides greater Scatternet functionality.

The major limitation with the implemented Scatternet Tree Network Structure was reliability. As the Scatternet bridging node is required to communicate in multiple Piconets while maintaining up to four RFCOMM connections it is utilising most (if not all) of the on board limited resources. Frequently during testing the Scatternet Bridging Node became unresponsive or favoured specific slave devices on the Piconet. These unexplainable events have been attributed to the limited resources of the BlueCore chip which was mainly designed for point to point communications.

As long as the throughput per sensor node is limited to a maximum of $3 \mathrm{kbps}$ and the number of nodes per Scatternet bridging node is limited to three, then the Scatternet Tree Network Structure provided reliable throughput results. This network structure is therefore appropriate in Bluetooth based wireless sensor networks. 


\subsubsection{Piconet Tree Bluetooth Network Structure Testing}

The Piconet Tree Network Structure is a cross between the Scatternet Tree Network Structure (described in 5.2.2 Scatternet Tree Bluetooth Network Structure Testing) and the standard Bluetooth Piconet (described in 5.1.3 Mobile Node Created Standard Piconet Bluetooth Network Structure Testing). The Piconet Tree Structure is a tree structure built from standard Bluetooth Piconets. This is done by the bridging nodes consisting of two Bluetooth radios. Creation and maintenance of the Piconet Tree Network Structure is similar to the Scatternet Tree Structure, this is handled by the master root node device. The BlueStack message sequence for standard RFCOMM based Bluetooth Piconets can be found in Appendix E - Bluetooth RFCOMM Message Sequence Chart. Commands and data are passed between the slave and master radios on the bridging node via serial communications. The stack architecture of the Piconet Tree Network Structure bridging nodes is shown in Figure 58 along with a standard Bluetooth slave wireless sensor node.

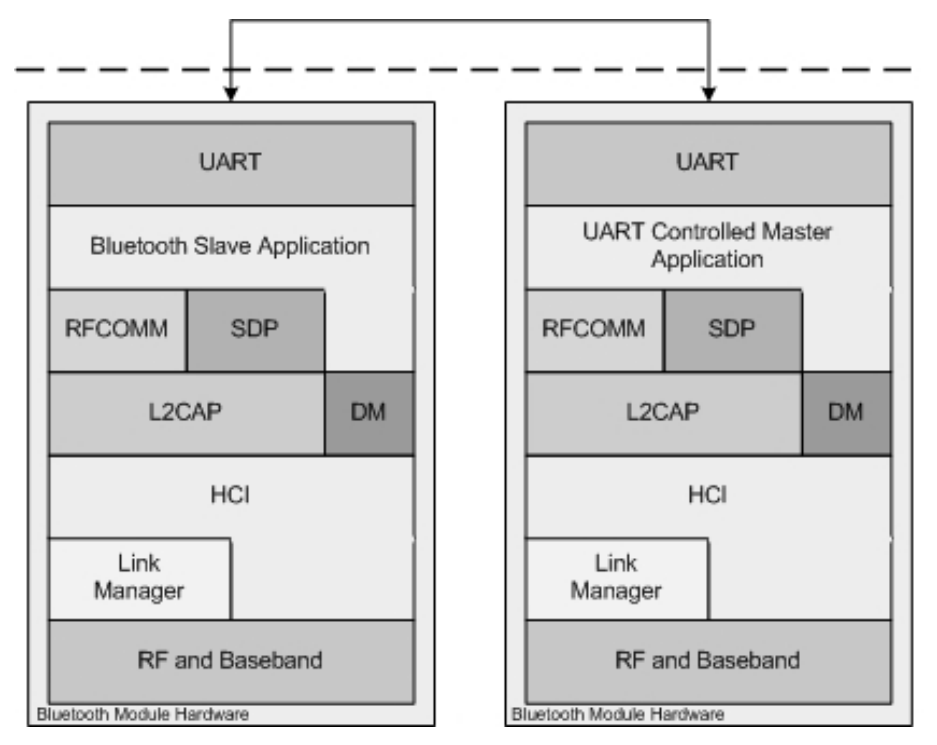

(a)

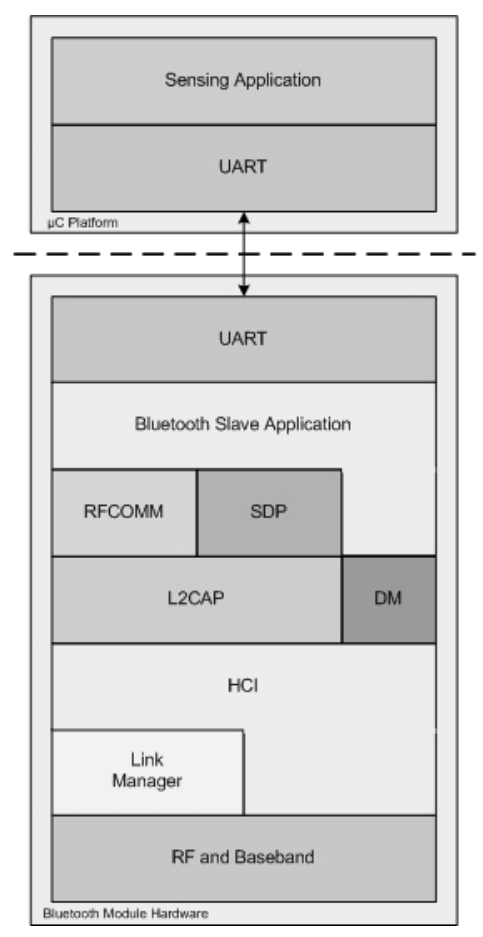

(b)

Figure 58: Bluetooth Piconet Tree Stack Architecture

(a) Piconet Tree Bridging Node Stack Architecture and (b) Piconet Tree Sensing Node Stack Architecture The testing results of a single Piconet Tree Bridging Node and a number of Piconet Tree Sensing Nodes appear below as Table 24 . 


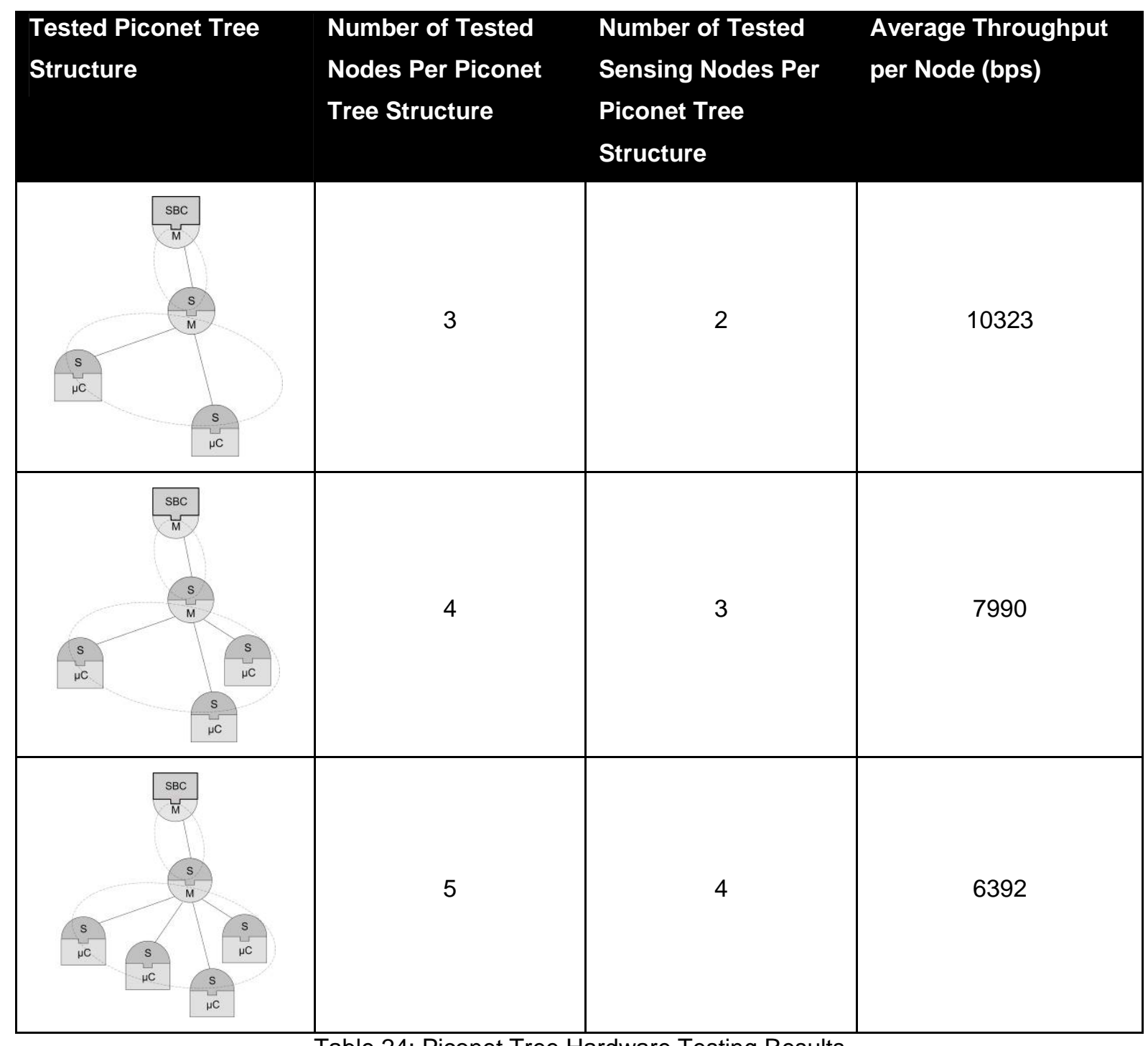

Table 24: Piconet Tree Hardware Testing Results

The testing results appearing in Table 24 were averaged over three throughput tests running for ten minutes each. This amount of testing resulted in a repeatable throughput per sensing node.

Testing the Bridging Node with more than four slaves resulted in unpredictable results with the master device in the Bridging Node becoming unresponsive. The major throughput limitation of Piconet Sharing Structure is the master radio on the Bridging Node. It is for this reason that Piconet coexistence will have little effect on the tested hardware results and extrapolation of the results in Table 24 will yield accurate results for a fully populated Piconet Tree Network Structure. Table 25 and Table 26 are the result of this extrapolation. 


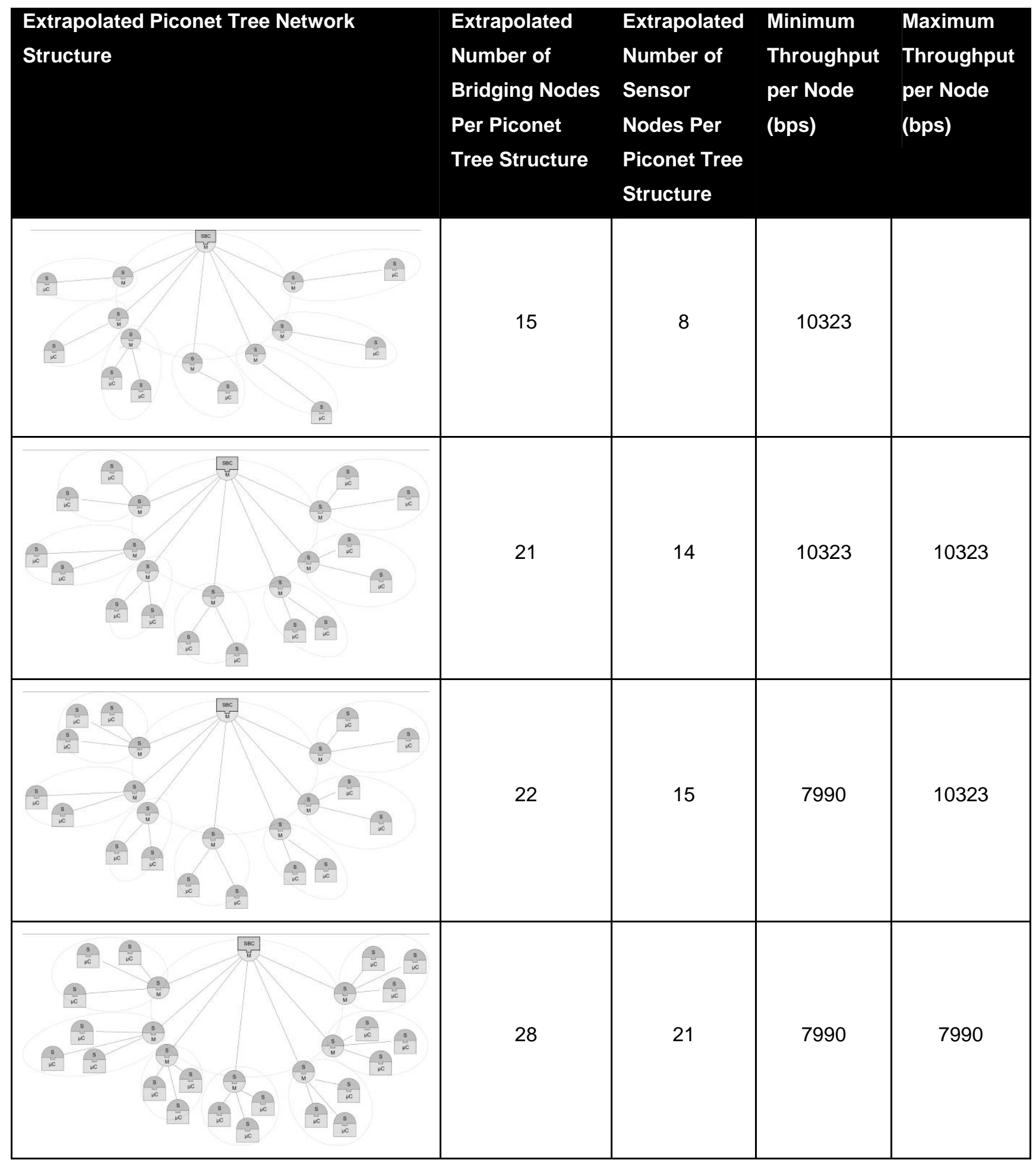

Table 25: Extrapolated Piconet Tree Hardware Testing Results - 107 - 


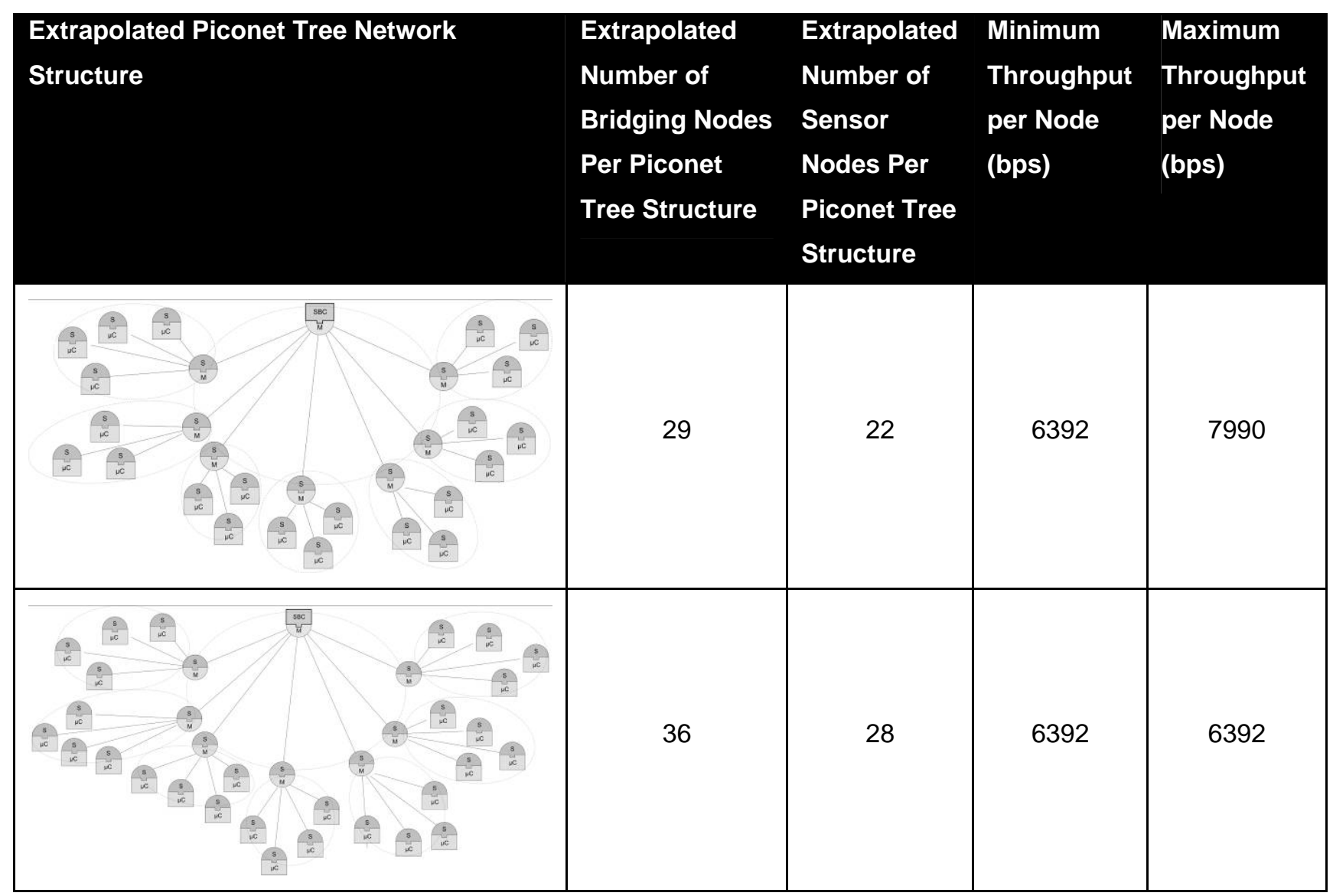

Table 26: Extrapolated Piconet Tree Hardware Testing Results

The extrapolated minimum throughput per node for the Piconet Tree Structure appears as Figure 59. The major limitation of the Piconet Tree Network Structure is that it requires non sensing bridging nodes as part of the network. These bridging nodes contain two Bluetooth radios and therefore do not support a sensor platform. 


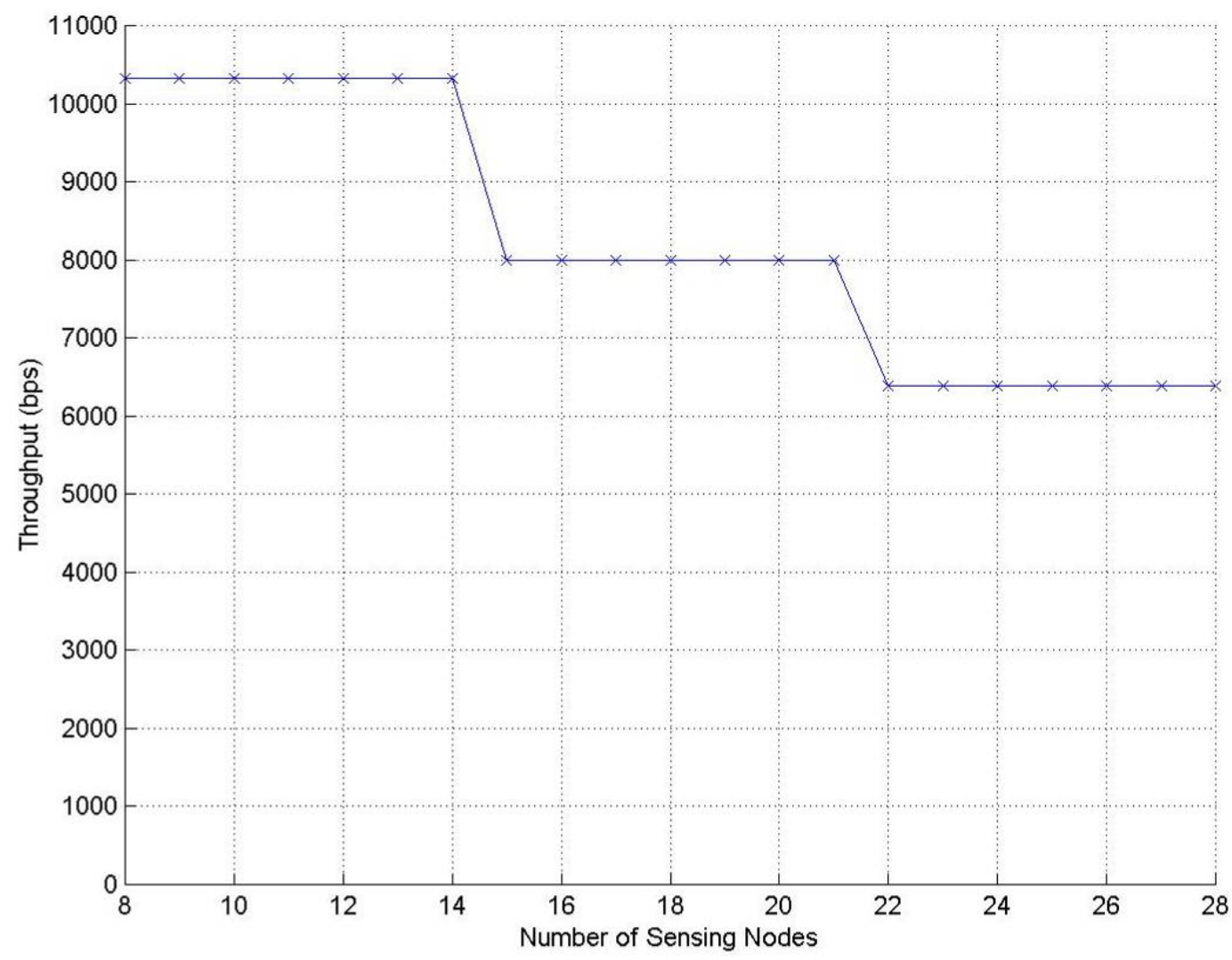

Figure 59: Extrapolated Bluetooth Piconet Tree Network Structure Minimum Throughput

The Piconet Tree Network Structure is most suitable for wireless sensing applications where multiple sensor platforms are on a single mobile object, so that the daughter nodes relay the data back to the bridging node which communicates back to the root node. If this network is tightly grouped class 2 Bluetooth devices can be used, which results in an extended battery life.

\subsubsection{Multiple Piconet Bluetooth Network Structure Testing}

The Multiple Piconet Bluetooth Network Structure creates multiple Piconets per Bluetooth root node by attaching multiple Bluetooth radios to the single board computer (SBC). The WinCE Bluetooth stack implementation is limited to a single Bluetooth radio, so the upper Bluetooth layers have been implemented on board the Bluetooth hardware (as part of the RFCOMM firmware build). The Piconet creation, management and data transfer is handled by serial commands to and from the SBC. While the SBC processor (Intel PXA255) has four UARTs, only two of the UARTs support the required $921.6 \mathrm{kbps}$ baud rate required for Bluetooth communications. A possible solution for this is to implement USB to serial conversion chips in hardware that support the full Bluetooth baud rate. The stack architecture of the root node in the Multiple Piconet Network Structure is shown in Figure 59. 


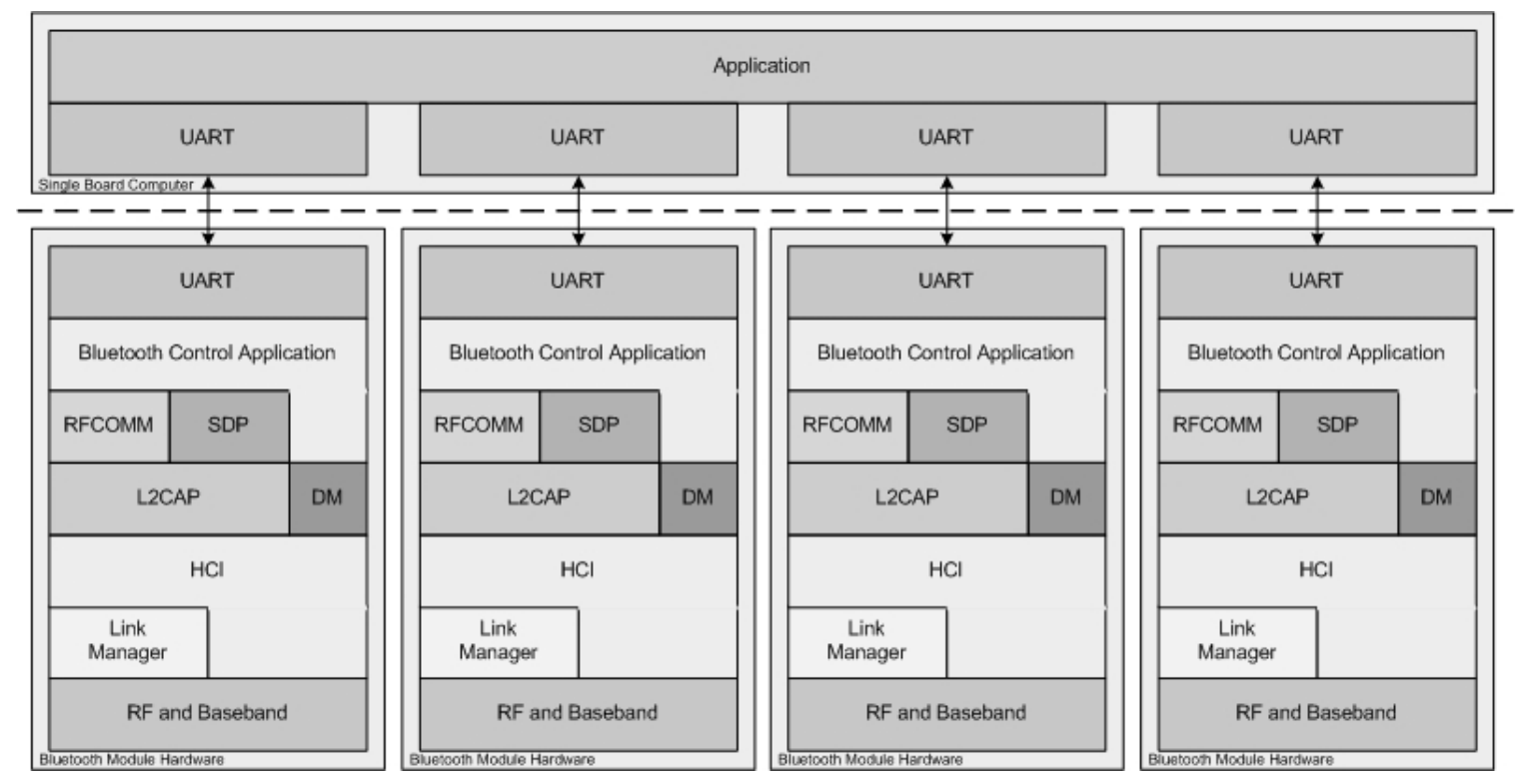

Figure 60: Bluetooth Multiple Piconet Network Structure Stack Architecture

The Multiple Piconet Network Structure uses the multiple Bluetooth radios to form RFCOMM based connections with the wireless sensor nodes. Appendix E outlines the BlueStack message sequence for RFCOMM based connections. The results of the hardware testing of a single radio in the Multiple Piconet Network Structure have been included as Table 27. The testing results appearing in Table 27 have been averaged over three tests, each running for ten minutes in length. 


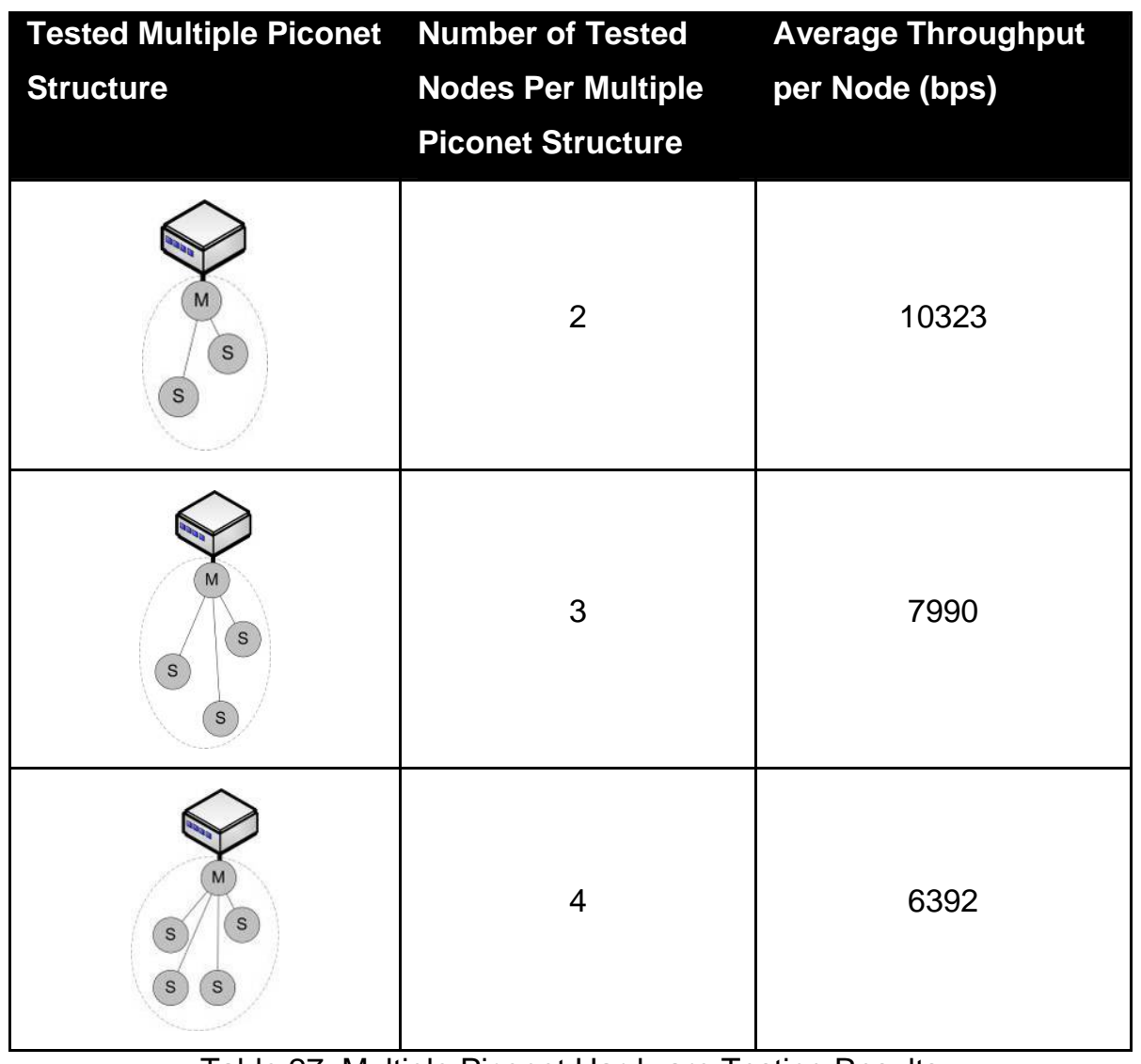

Table 27: Multiple Piconet Hardware Testing Results

The results in Table 27 have been extrapolated to provide the overall network throughput for a Multiple Piconet Network Structure with four Bluetooth radios. The effect of Piconet coexistence has not been taken into account in the extrapolation of results due to the network structures throughput bottleneck being the master Bluetooth devices. Table 28 is a summary of the extrapolated testing results for the Multiple Piconet Network Structure. 


\begin{tabular}{|c|c|c|c|c|}
\hline $\begin{array}{l}\text { Extrapolated Multiple Piconet } \\
\text { Network Structure }\end{array}$ & $\begin{array}{l}\text { Actual Number of } \\
\text { Tested Nodes Per } \\
\text { Multiple Piconet } \\
\text { Structure }\end{array}$ & $\begin{array}{l}\text { Extrapolated } \\
\text { Number of Nodes } \\
\text { Per Multiple } \\
\text { Piconet Structure }\end{array}$ & $\begin{array}{l}\text { Minimum } \\
\text { Throughput } \\
\text { per Node } \\
\text { (bps) }\end{array}$ & $\begin{array}{l}\text { Maximum } \\
\text { Throughput } \\
\text { per Node } \\
\text { (bps) }\end{array}$ \\
\hline $\begin{array}{llll}(s & & s & s \\
& s & s & \end{array}$ & 2 & 8 & 10323 & 10323 \\
\hline$(S) S S_{S}^{M} \int_{S}^{M}(S)$ & 3 & 9 & 7990 & 10323 \\
\hline 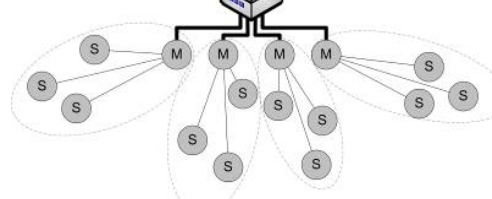 & 3 & 12 & 7990 & 7990 \\
\hline 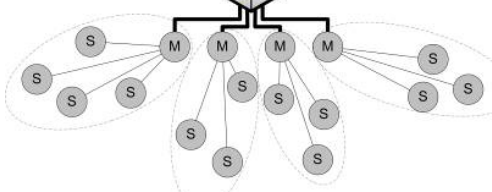 & 4 & 13 & 6392 & 7990 \\
\hline 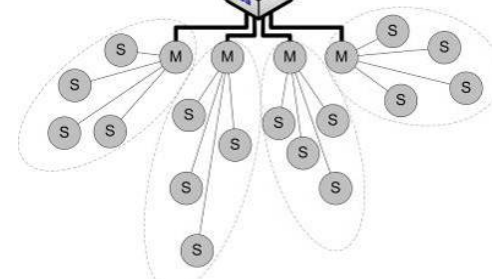 & 4 & 16 & 6392 & 6392 \\
\hline
\end{tabular}

Table 28: Extrapolated Multiple Piconet Hardware Testing Results 
The minimum throughput per wireless node can be seen in Figure 61. These throughput results are for four Bluetooth radios per Bluetooth root node. Increasing the number of attached radios will increase the throughput per node, but will also cause a greater amount of interference from the coexisting Piconets.

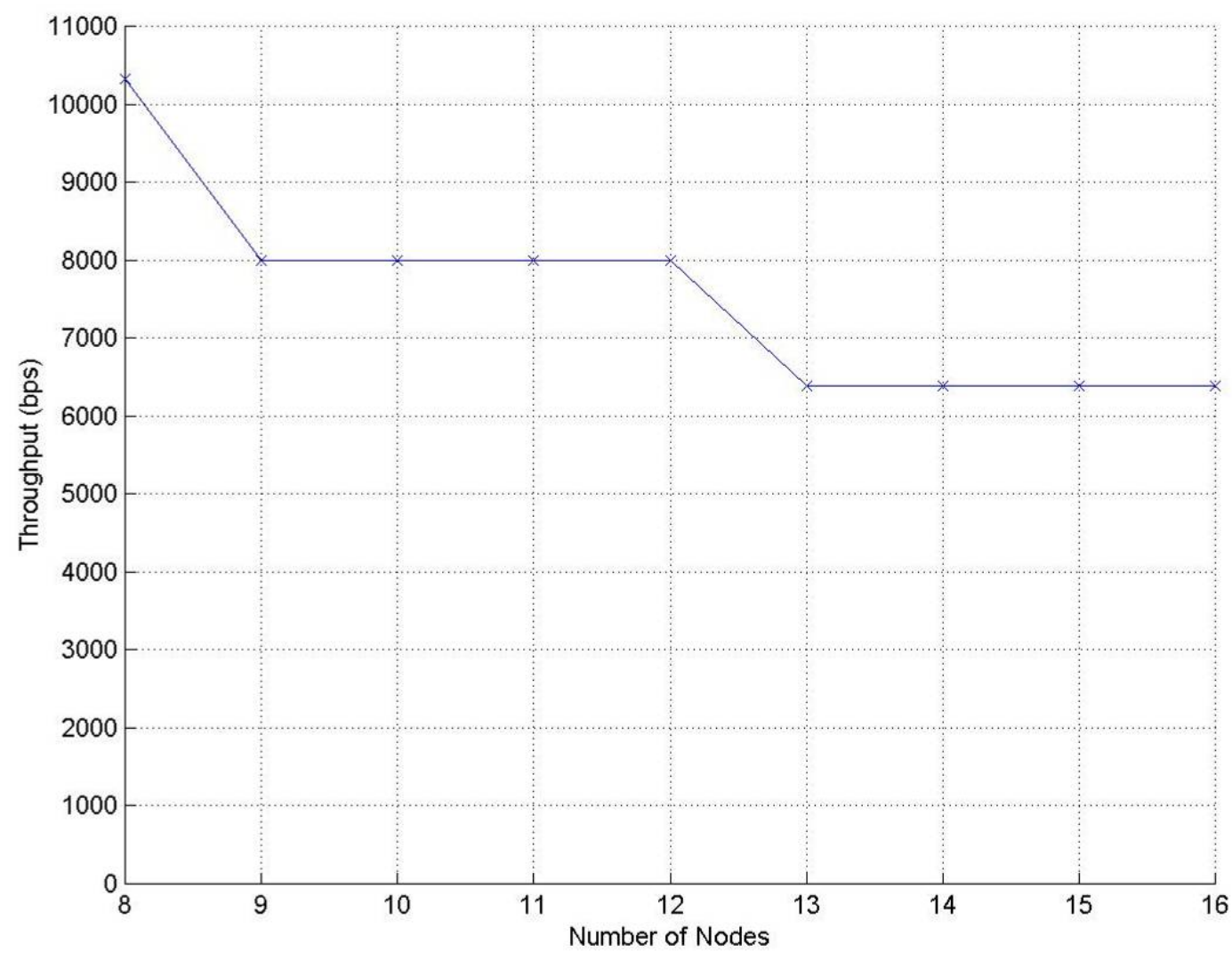

Figure 61: Extrapolated Bluetooth Multiple Piconet Network Structure Minimum Throughput

The major limitation of the Multiple Piconet Network Structure is the reduced throughput due to the upper Bluetooth layers of the stack being implemented on board the CSR BlueCore Chipset. A more practical solution is to write an additional Bluetooth stack layer that would allow multiple Bluetooth physical radios to access the same upper layer stack. Figure 62 shows the location of the proposed stack layer in the Bluetooth stack architecture. 


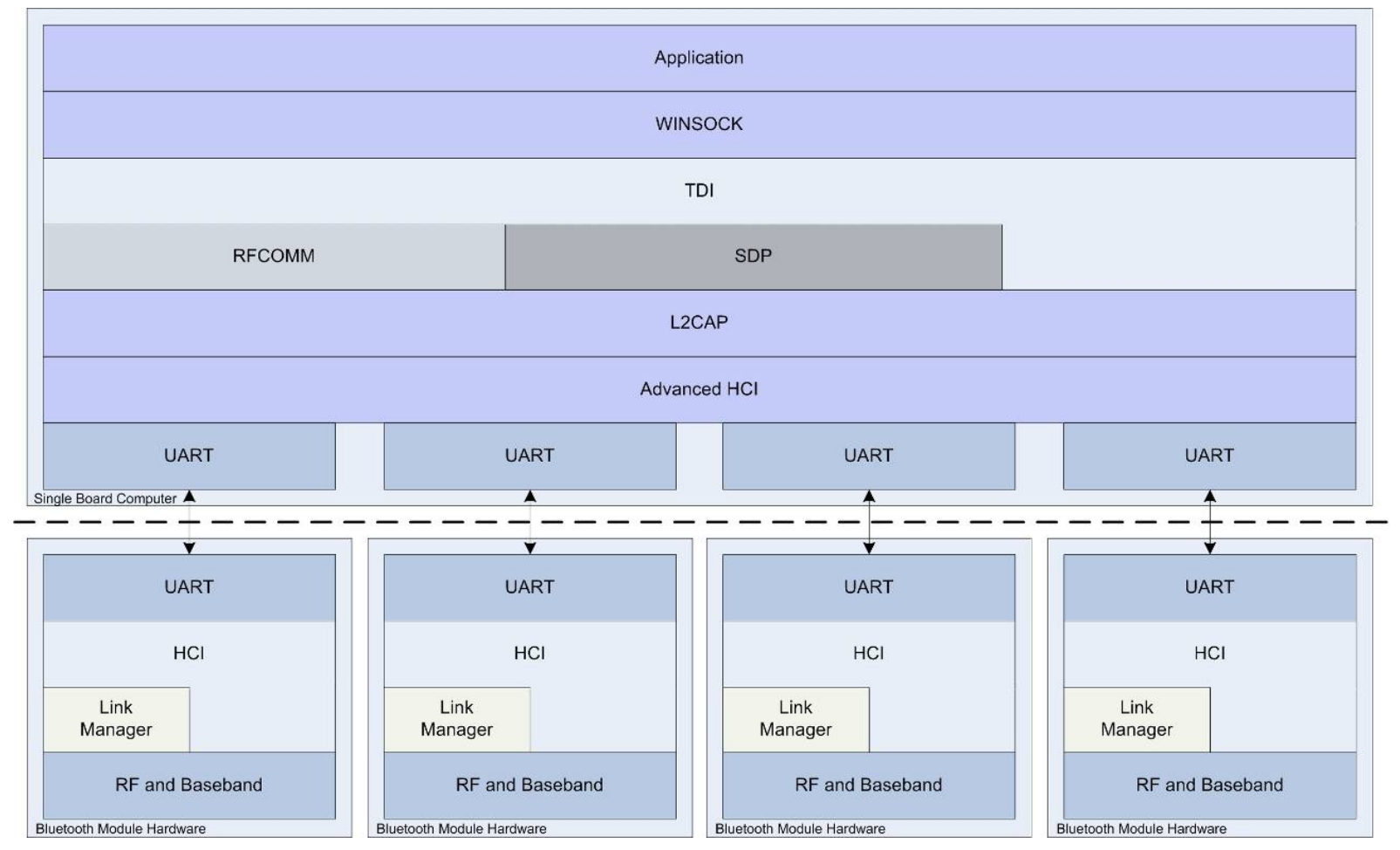

Figure 62: Proposed Advanced Host Control Interface Bluetooth Stack Layer

This advanced host control interface layer $(\mathrm{AHCl})$ would allow more than seven Bluetooth devices to actively communicate with a Bluetooth access point without imposing any throughput limitations or function limitations on the forms of communication. To the authors knowledge no such stack layer has been proposed or implemented at the time of this works publication and as such is an area for on going research.

\subsection{Bluetooth Wireless Sensor Network Testing Summary}

The implementation of the Bluetooth Network Structures with available hardware has highlighted various limitations with the test hardware. The wireless sensor application that the network has been designed for requires a total throughput of 4500 bps (three channels of 10 bit data at 150 times a second). Each network structure has been compared to this throughput requirement.

When constructing a standard Bluetooth Piconet with the embedded Bluetooth modules (RFCOMM firmware build using the CSR BC02 - Ext chipset), the device is not able to successfully maintain a Piconet with the Bluetooth standard of seven slaves. The maximum number of active slaves that could be sustained was four. 
Standard Piconet hardware testing Bluetooth also proved that the CSR BC02 chipset had an undocumented limit of a ten devices parked at a time. This limited the Piconet Sharing Structures usefulness in this application. To be able to successfully implement the Piconet Sharing Bluetooth Network Structure additional Bluetooth hardware must be sourced that can support a greater number of parked slaves on a Piconet. The Piconet Sharing Network Structure was still tested in hardware by extrapolating the number of nodes per structure. With this extrapolation the Piconet Sharing Structure was able to provide the required data rate for more than 20 nodes.

Testing of the Scatternet Tree network structure demonstrated that the limited resources of the Bluetooth Chipset used was unable to reliably support Scatternet operation (using the RFCOMM firmware build). Testing was completed using the hardware with a maximum of three slaves per bridging node Piconet. Due to the hardware resource limitation the throughput results for the Scatternet Tree were much smaller than expected from the corresponding Bluetooth Simulation. Control over the bridging node Piconet swapping was not successfully achieved and this also impacted on the low throughput results. Even with these limitations the Scatternet Tree Structure was able to provide the required data rate for up to 20 nodes.

Piconet Tree hardware testing demonstrated that a stable tree network could be constructed if two Bluetooth radios were used for the bridging node. A maximum of four slaves could be connected to the bridging node, due to the limitations of using the Bluetooth modules as the master of the Piconet. The Bluetooth modules used only had a single UART so the bridging nodes could not support wireless sensor platforms. This structure is appropriate for wireless sensing applications that require multiple sensor platforms on a single athlete or object. The Piconet Tree network structure provided the required sensor platform throughput for over 30 nodes.

The Multiple Piconet Bluetooth network structure creates multiple Piconets controlled by the root node by using multiple Bluetooth radios. The WinCE based Bluetooth Access Points used only supported a single Bluetooth radio. Therefore the upper layers of the Bluetooth stack were implemented on board the Bluetooth modules and controlled using serial commands. This meant that a maximum of four slaves could be connected per Piconet (due to the chipset limitation). Testing of the Multiple Piconet network structure showed that over 20 nodes were supported at the required wireless sensor platform data rate. Creating an Advanced Host Controller Interface (AHCl) Bluetooth layer to support more than one Bluetooth radio would allow much higher data rates to be achieved for this structure.

The testing of the developed network structures in hardware allowed real world throughput results to be obtained. Many of the tests performed in this body of work have not be attempted before (with this hardware) and therefore are an original contribution to the research field. Several hardware limitations have been discovered. 
All of the proposed structures proved useful for different wireless sensing applications. These results serve as a proof of concept and showed that Bluetooth based wireless sensor networks can function with more than seven active slaves at a time. Combining the described network structures with multiple root nodes allows Bluetooth wireless sensor networks to be created that support node requirement of more than 50 nodes. 


\section{Conclusion}

The proposed Bluetooth network structures have been developed to expand the usefulness of the Bluetooth specification for use in wireless sensor networks. These structures have been compared via simulated results and have been tested using existing Bluetooth hardware. Each structure has inherit advantages and disadvantages, with each being useful for different sensing applications. Combinations of the developed structures can be implemented for different wireless sensing applications. While the developed network structures have been implemented for using in wireless sensing applications, the same principles can be applied for other applications. Expanding Bluetooth communications beyond the eight device node limit expands usefulness of the Bluetooth specification for applications requiring more than eight devices.

The Bluetooth wireless protocol has been investigated for use in wireless sensor networks for sports monitoring applications. This body of work has produced several outcomes including novel Bluetooth network structures that increase the usefulness of the Bluetooth specification. A thorough literature review examined current and future wireless network standards highlighting several appropriate alternatives to the Bluetooth specification for wireless sensing applications. A critical review of the Bluetooth specification was undertaken to determine techniques for extending the node limitations. Four Bluetooth network structures have been developed for wireless sensing applications. A MATLAB based simulation tool was written to calculate the Bluetooth communications throughput under various conditions. This simulation was modified to calculate theoretical throughput values for the developed network structures. Hardware testing of the developed Bluetooth network structures highlighted several limitations (some undocumented) in the hardware and software Bluetooth implementations.

This chapter summarises the work completed and the measurable research outcomes. Hardware and software limitations have been summarised and should be taken into consideration when implementing the wireless sensor applications with tested Bluetooth hardware. Future work has been proposed to increase the stability of the developed network structures and further increase the usefulness of the Bluetooth specification for wireless sensor networks.

\subsection{Project Summary}

This body of work has produced several major outcomes that are useful in the many fields not just for use in sports monitoring. Many of the outcomes and results achieved by this research have not been accomplished by any other research, some of which has resulted in conference publications included as Appendix I, J and $\mathrm{K}$ and referenced as $[69,70,71]$. The major outcomes of this body of work are: 
- Provided a thorough investigation into current and future wireless standards that affect the area of wireless sensor networks

- Developed a cutdown MATLAB simulation of communications on a Bluetooth Piconet

- Thorough testing of selected Bluetooth hardware, exposing undocumented limitations in Bluetooth hardware implementations

- Designed, simulated and experimentally tested four novel Bluetooth Network Structures

- Proposed Advanced Host Control Interface (AHCl) Bluetooth stack layer that allows multiple Bluetooth radios.

\subsection{Limitations Encountered}

During the course of the Bluetooth testing several major hardware and software limitations were discovered. The major limitations were:

- Bluetooth hardware parking limitation.

It was discovered that the Bluetooth radios used had an undocumented limit of ten parked devices at any time. This effected the implementation of the Piconet Sharing Network Structure.

- Bluetooth hardware instability when creating Scatternets using onboard upper Bluetooth stack layers (RFCOMM firmware build).

It was shown that master / slave bridging nodes could only support a limited number of slaves (up to three) before the limited resources of the BlueCore chipset was reached. Even below this limit Scatternet operation was unreliable.

- Throughput limitation when using Bluetooth hardware with integrated stack layers as master of piconet.

It was shown that the Bluetooth hardware using onboard upper Bluetooth stack layers could only support a limited number of slaves (up to four) when creating piconets.

- WinCE Bluetooth stack implementation only supports a single Bluetooth radio.

\subsection{Future Work}

While this body of research has already provided several notable outcomes, like any body of work there is always on going areas of work. Areas of future work on this project include:

- Integration of new features into the Bluetooth simulation software

- Rewrite of firmware for release (currently proof of concept stage) 
- Investigation into further Bluetooth hardware (that will provide better Scatternet support)

- Implementation of an advanced host control interface (AHCl) Bluetooth stack layer

\subsection{Bluetooth Wireless Sensor Network Recommendations}

Several Bluetooth Network Structures have been developed for implementation with multiple root nodes in a wireless sensing environment. Each Network Structure has advantages and limitations and is suitable for different wireless sensor applications.

The Piconet Sharing Network Structure provided the highest overall throughput of any of the tested network structures. However this comes at the cost of having a high latency between active communication periods. If a node is unable to buffer the sensor data during the inactive times, then buffer overrun will occur and data will be lost. This network structure was not possible to implement with the current Bluetooth hardware (CSR BlueCore02) as this had an unspecified limit of up to ten parked device on a piconet at a time. This limit is not in accordance with the Bluetooth specification and alternate Bluetooth hardware should not suffer this problem.

The Scatternet Tree Network Structure allowed a tree style network to be set up with single Bluetooth radios. However hardware testing showed that the Bluetooth hardware was unable to create stable Scatternets (with the full Bluetooth stack onboard). An additional processor must be used for the Bluetooth upper layers or additional Bluetooth hardware must be sought that has greater Scatternet capabilities.

The Piconet Tree Network Structure provided a reliable tree style network to be created. The limitation of this structure is that it requires non-sensing routing nodes that consist of two Bluetooth radios. This network structure is ideally suited to applications where small numbers of sensor nodes are grouped together in a local area (such as multiple sensors on a single athlete). In this way the sensor nodes can relay the sensor data to the routing node which can transmit the data back to the fixed route node. If the range is short enough the power on the sensor nodes can be reduced to further increase the battery life time.

The Multiple Piconet Network Structure allowed the root node (Bluetooth access point) to connect to more than seven active slaves at a time. The Bluetooth implementation part of the WinCE operating system used on the root node only supports a single Bluetooth radio at a time. For this reason the upper Bluetooth layers were utilised on the embedded Bluetooth hardware and controlled via serial commands. Implementing an advanced host controller interface $(\mathrm{AHCl})$ that is compatible with multiple Bluetooth radios will allow Bluetooth access points to seamlessly connect to greater than seven active slaves at a time without any functional restrictions. 
The Piconet Sharing and Multiple Piconet Structures are single-hop networks meaning that each wireless sensor node has a direct link to the root node and is only responsible for the one wireless sensor platforms data. In a noisy environment where temporary node drop outs are frequent this style network is preferable over multi-hop networks as they require less network reconfiguration. While the Scatternet Tree and Piconet Tree Network structures are limited to be in range of the root node (for node discovery purposes), they still suffer this problem as they are essentially multi-hop networks as some nodes act as routing nodes for the sensor data to get back to the root node.

Bluetooth is an established wireless technology that has plenty of industry support and hardware vendors. As shown in this body of work Bluetooth can be appropriate technology in wireless sensor networks, but further research is required to fine tune the described network structures. The outcomes demonstrated in this body of work are proof of concept results, and dedicated Bluetooth stack implementations are required to take advantage of the functionality described in this work. 


\section{References}

[1] Bluetooth SIG, Specification of the Bluetooth System, Volume 1: Core, Version 1.1, 2001

[2] D. A James, N. Davey and L. Gourdeas, A modular integrated platform for microsensor applications, Procedings of SPIE vol 5274, Microelectronics: Design, Technology and Packaging, Bellingham, WA, 2004

[3] Accton White Paper, 802.11: Wireless Networking. A Guide to Wireless Networks and 802.11x Technologies and Standards, viewed online 28 March 2006, http://www.accton.com/homepage/main3/whitepaper/paper_2.htm

[4] IEEE Computer Society, IEEE Std 802-2001 IEEE Standards for Local and Metropolitan Area Networks: Overview and Architecture, The Institute of Electrical and Electronics Engineers Inc, December 2001

[5] Wikipedia, the free encyclopedia. IEEE 802.11, viewed online 28 March 2006, http://en.wikipedia.org/wiki/802.11

[6] Atheros Communications White Paper, Super G Maximising Wireless Performance, viewed online 11 April 2006, http://www.super-g.com/atheros_superg_whitepaper.pdf

[7] Wikipedia, the free encyclopedia. IEEE 802.15, viewed online 11 April 2006, http://en.wikipedia.org/wiki/802.15

[8] IEEE Computer Society, 802.15.3 IEEE Standard for Information technology - Telecommunications and information exchange between systems - Local and metropolitan area networks - Specific requirements, Part 15.3: Wireless Medium Access Control (MAC) and Physical Layer (PHY) Specifications for High Rate Wireless Personal Area Networks (WPANs), The Institute of Electrical and Electronics Engineers Inc, September 2003

[9] Bluetooth Demystified, Nathan J. Muller, 2001, McGraw Hill

[10] J. Scarisbrick and P. Goodridge, Fourth Quarter and Full Year 2005 Financial Results, Cambridge Silicon Radio, 28 February, 2006, viewed online 28 March 2006, http://ir.csr.com/csr/fininfo/presentations/prelim_results05/2006_02_28_pr.pdf

[11] WiMedia Alliance, WiMedia Alliance - Home Page, viewed online 29 September 2006, http://www.wimedia.org/en/index.asp

[12] UWB Forum, UWB Forum, viewed online 29 September 2006, http://www.uwbforum.org/

[13] Engadget, Freescale and Motorola ditch the UWB Forum, Posted Apr 7th 2006, viewed online 18 April 2006, http://www.engadget.com/2006/04/07/freescale-and-motorola-ditch-the-uwb-forum/

[14] ECMA International, Standard ECMA-368 High Rate Ultra Wideband PHY and MAC Standard, December 2005 
[15] Wikipedia, the free encyclopedia. Ultra Wideband, viewed online 18 April 2006, http://en.wikipedia.org/wiki/Ultra_wideband

[16] IEEE Computer Society, 802.15.4 IEEE Standard for Information technology - Telecommunications and information exchange between systems - Local and metropolitan area networks - Specific requirements, Part 15.4: Wireless Medium Access Control (MAC) and Physical Layer (PHY) Specifications for Low-Rate Wireless Personal Area Networks (LR-WPANS), The Institute of Electrical and Electronics Engineers Inc, October 2003

[17] IEEE Computer Society, IEEE Std 802-2001 IEEE Standards for Local and Metropolitan Area Networks: Overview and Architecture, The Institute of Electrical and Electronics Engineers Inc, December 2001

[18] Figure 8 Wireless, ZigBee Implementer's Guide Version 1.1, Figure 8 Wireless, Inc, San Diego, CA, May 2005

[19] Wikipedia, the free encyclopedia. ZigBee, viewed online 11 April 2006, http://en.wikipedia.org/wiki/zigbee

[20] IEEE 802.15.4a Task Group, IEEE 802.15 WPAN Low Rate Alternative PHY Task Group 4a (TG4a), viewed online 11 April 2006, http://www.ieee802.org/15/pub/TG4a.html

[21] T. Cooklev, Wireless Communication Standards: A study of IEEE 802.11, 802.15 and 802.16, IEEE Standards Wireless Networks Series, Standards Information Network, IEEE Press 2004

[22] T. F. Budinger, Biomonitoring with Wireless Communications, Annual Review of Biomedical Engineering, Vol. 5: 383-412 (Volume publication date August 2003)

[23] K. Zhang, P. Werner, M. Sun, X. Pi-Sunyer, C. N. Boozer, Measurement of human daily physical activity, Journal of Obesity Research, Volume 11 Number 1, Pages 33 - 40, 2003

[24] B. Heller, S. Haake, The Smart Sensor Project: Empowering the User, Conference Proceedings of Asia-Pacific Congress on Sports Technology, Tokyo Institute of Technology, September 2005.

[25] D. A. James, I. Jaffari, A System for Multi-Limb Motion Monitoring Using Tri-Axial Accelerometer Nodes on a Wireless Network, Conference Proceedings of Asia-Pacific Congress on Sports Technology, Tokyo Institute of Technology, September 2005.

[26] J. J. Kavanagh, S. Morrison, D. A. james, R. Barrett, Reliability of Segmental Accelerations Measured Using a New Wireless Gait Analysis System, Elservier Journal of Biometrics, Accepted 20 September 2005

[27] K. Zhang, Y. Li, R. Grenfell, A. Hahn, C. Gore, T. Rice , Developing Prototype Multisensor GPS Rower Monitoring and Coaching Systems for Olympics, FIG Working Week, Intercontinental Athenaeum Athens, Athens, Greece, May 2004 
[28] Bluetooth SIG, Specification of the Bluetooth System, Specification Volume 2: Core System Package, Version $2.0+$ EDR, 2004

[29] Cambridge Silicon Radio, BlueCore 2-External RFCOMM 1.1v16.8.3 Software Release Note, 2003

[30] Bluetooth SIG, Specification of the Bluetooth System, Core System Package [Controller Volume], Version 1.2, 2003

[31] S. Baatz, M. Frank, R. Göpffarth, D. Kassatkine, P. Martini, M. Schetelig, A. Vilavaara, Handoff Support for Mobility with IP over Bluetooth, Conference Proceedings of 25th Annual Conference on Local Computer Networks (LCN'00), Tampa, FL, USA, November 2000

[32] W. H. Lee, Y. Joo, K. H. Tchah, Y. Kim, D. Eom, Handoff Provisioning in Bluetooth Wireless Personal Area Networks, IEEE Transactions on Consumer Electronics, Vol. 49, No. 4, November 2003

[33] S. Chung, H. Yoon, J. Cho, A Fast Handoff Scheme For IP over Bluetooth, Conference Proceedings International Conference on Parallel Processing Workshops, 2002

[34] W. H. Lee, Y. Joo, K. H. Tchah, Y. Kim, D. Eom, Handoff Provisioning in Bluetooth Wireless Personal Area Networks, IEEE Transactions on Consumer Electronics, Vol. 49, No. 4, November 2003

[35] S. Baatz, M. Frank, R. Göpffarth, D. Kassatkine, P. Martini, M. Schetelig, A. Vilavaara, Handoff Support for Mobility with IP over Bluetooth, Conference Proceedings of 25th Annual Conference on Local Computer Networks (LCN'00), Tampa, FL, USA, November 2000

[36] Personal Area Networking Working Group, Personal Area Network Profile, Version 1.0, February 2003

[37] S. Feldmann, K. Kyamakya, A. Zapater, Z. LuAn, Indoor Bluetooth-based Positioning System: Concept, Implementation and Experimental Evaluation, viewed online 9 May 2006, http://projekte.|3s.uni-hannover.de/pub/bscw.cgi/0/25772

[38] J. Hallberg, M. Nilsson, K. Synnes, Positioning with Bluetooth, 10th International Conference on Telecommunications, 2003

[39] T. Salonidis, P. Bhagwat, L. Tassiulas and R. LaMaire. Distributed Topology Construction of Bluetooth Personal Area Networks. IEEE Infocom, 2001

[40] T. Melodia and F. Cuomo. Ad hoc networking with Bluetooth: key metrics and distributed protocols for Scatternet formation. Elsevier's Journal of Ad Hoc Networks, Volume 2, Issue 2, Pages 185-202. April 2004.

[41] C. Law, A. K. Mehta, and K.Y. Siu. Performance of a new Bluetooth scatternet formation protocol. Proceedings of MobiHoc'01, Long Beach, CA. October 2001.

[42] Y. Kawamoto, V.W.S. Wong, V.C.M. Leung, A two-phase scatternet formation protocol for Bluetooth wireless personal area networks, Proceedings of Conference IEEE Wireless Communications and Networking, 2003 
[43] Z. Wang, R. J. Thomas, Z. Haas, Bluenet - a New Scatternet Formation Scheme, Proceedings of 35th Annual Hawaii International Conference on System Sciences, 2002

[44] G. V. Z'aruba, S. Basagni, I. Chlamtac, Bluetrees - Scatternet Formation to Enable Bluetooth-based Ad Hoc Networks, Proceedings of IEEE International Conference on Communications, 2001

[45] P. Flittner, IEEE P1451.5 Wireless Sensor Interface Working Group Comments on use of Bluetooth Technology, IEEE P1451.5 Wireless Sensor Interface Working Group, viewed online 9 May 2006, http://grouper.ieee.org/groups/1451/5/,

[46] IEEE 11073 Working Group, Guidelines for the use of RF wireless technologies, IEEE 11073-00101 Health informatics - Point-of-care medical device communication, viewed online 9 May 9, 2006, http://www.ieee1073.org/standards/11073-00101/11073-00101.html

[47] K. Bult, A. Burstein, D. Chang, M. Dong, M. Fielding, E. Kruglick, J. Ho, F. Lin, T.H. Lin, W.J. Kaiser, H. Marcy, R. Mukai, P. Nelson, F. Newberg, K.S.J. Pister, G. Pottie, H. Sanchez, O.M. Stafsudd, K.B. Tan, C.M. Ward and S. Xue, J. Yao. Low Power Systems for Wireless Microsensors, Proceedings of the 1996 International Symposium on Low Power Electronics and Design. Monterey, CA, USA. pages 17-22. August 1996.

[48] R. Min, M. Bhardwaj, S.H. Cho, A. Sinha, E. Shih, A. Wang, and A.P. Chandrakasan. Low-Power Wireless Sensor Networks. The 14th International Conference on VLSI Design (VLSID '01), 2001

[49] J. Hill, R. Szewczyk, A. Woo, S. Hollar, D. Culler, and K. Pister. System architecture directions for networked sensors. In Tenth International Conference on Architectural Support for Programming Languages and Operating Systems, pages 93 -104, November 2000.

[50] J. M. Kahn, R. H. Katz and K. S. J. Pister. Next century challenges: Mobile networking for 'smart dust'. Proceedings of International Conference on Mobile Computing and Networking in Seattle, pages 271-278, 1999

[51] I. Chatzigiannakis, T. Dimitriou, M. Mavronicolas, S. Nikoletseas and P. Spirakis, A Comparative Study of Protocols for Efficient Data Propagation in Smart Dust Networks. 9th International Euro-Par Conference on Parallel Processing. Klagenfurt, Austria. Pages1003 - 1016. August 26-29, 2003

[52] Crossbow Technology Inc., Motes, Smart Dust Sensors, Wireless Sensor Networks, viewed online 29 July 2006, http://www.xbow.com/Products/Wireless_Sensor_Networks.htm

[53] Berkeley UC, Tiny OS Community Forum, An open-source OS for the networked sensor regime, viewed online 29 July 2006, http://www.tinyos.net/

[54] Intel Mote strategic research project, Intel Mote, viewed online 23 May 2006, http://www.intel.com/research/exploratory/motes.htm

[55] BTnodes - A Distributed Environment for Prototyping Ad Hoc Networks, viewed online 23 May 2006, http://www.btnode.ethz.ch/ 
[56] M. Leopold, M. B. Dydensborg, P. Bonnet, Bluetooth and Sensor Networks: A Reality Check, Proceedings of the 1st international conference on Embedded networked sensor, Los Angeles, California, USA, 2003

[57] G. V. Z'aruba, S. Basagni, I. Chlamtac, Bluetrees - Scatternet Formation to Enable Bluetooth-based Ad Hoc Networks, Proceedings of IEEE International Conference on Communications, 2001

[58] J. Beutel, M. Dyer, L. Meier, L. Thiele, Scalable Topology Control for Deployment-Support Networks, Fourth International Symposium on Information Processing in Sensor Networks (IPSN), April 2005

[59] W. H. Lee, Y. Joo, K. H. Tchah, Y. Kim, D. Eom, Handoff provisioning in Bluetooth wireless personal area networks, IEEE Transactions on Consumer Electronics, Volume: 49, Issue: 4, November 2003

[60] A. Kumarm, BlueHoc: Bluetooth Performance Evaluation Tool, viewed online 13 July 13, 2006, http://bluehoc.sourceforge.net/

[61] OPNET Technologies, OPNET Making Networks and Applications Perform, viewed online 13 July 2006, http://www.opnet.com/

[62] Highland Systems, Bluetooth Simulation Model Suite for OPNET, viewed online 13 July 2006, www.highsys.com/products/Suitetooth.pdf

[63] The MathWorks, MATLAB and Simulink for Technical Computing, viewed online 13 July 2006, http://www.mathworks.com/

[64] Intrinsyc Software, Intrinsyc Software - Device Management, CerfBoard 255 Developer's Kit, viewed online 30 July 2006, http://www.intrinsyc.com/products/mob_ref_sys/cerfboard_255_se/

[65] CC\&C Technologies Inc., CC\&C Bluetooth Modules, viewed online 11 April 2005, http://www.ccandc.com.tw/product/bt-module.htm

[66] Microsoft Developer Network, Bluetooth Stack Implementation Considerations, viewed online 30 July 2006,http://msdn.microsoft.com/library/default.asp?url=/library/enus/wceddk40/html/cxreflmplementationConsiderations.asp

[67] Cambridge Silicon Radio, BlueCore HCIStack1.1v14.8 Software Release Note, October 2002

[68] Cambridge Silicon Radio, BlueCore Scatternet Support, August 2005

[69] M. J. Fraser, D. A. James and D. V. Thiel, Wireless Network Topology for Monitoring Mobile Agents, Proceedings of SPIE vol 5651 Biomedical Applications of Micro- and Nanoengineering II, Sydney, NSW, 2004

[70] M. J. Fraser, D. A. James and D. V. Thiel, Innovative techniques for extending the range and node limits in Bluetooth-based wireless sensor networks, Proceedings of SPIE vol 6035 Microelectronics: Design, Technology and Packaging II, Brisbane, QLD, 2005 
[71] D. A. James and M.J. Fraser, The application of wireless technology and sensors for sporting applications, The Japan Society of Mechanical Engineers (Bioengineering Division), Sports Engineering Symposium, Kanazawa, 2006 (accepted for publication) 
Appendix A: Standard Piconet Bluetooth Network Structure Simulation Code

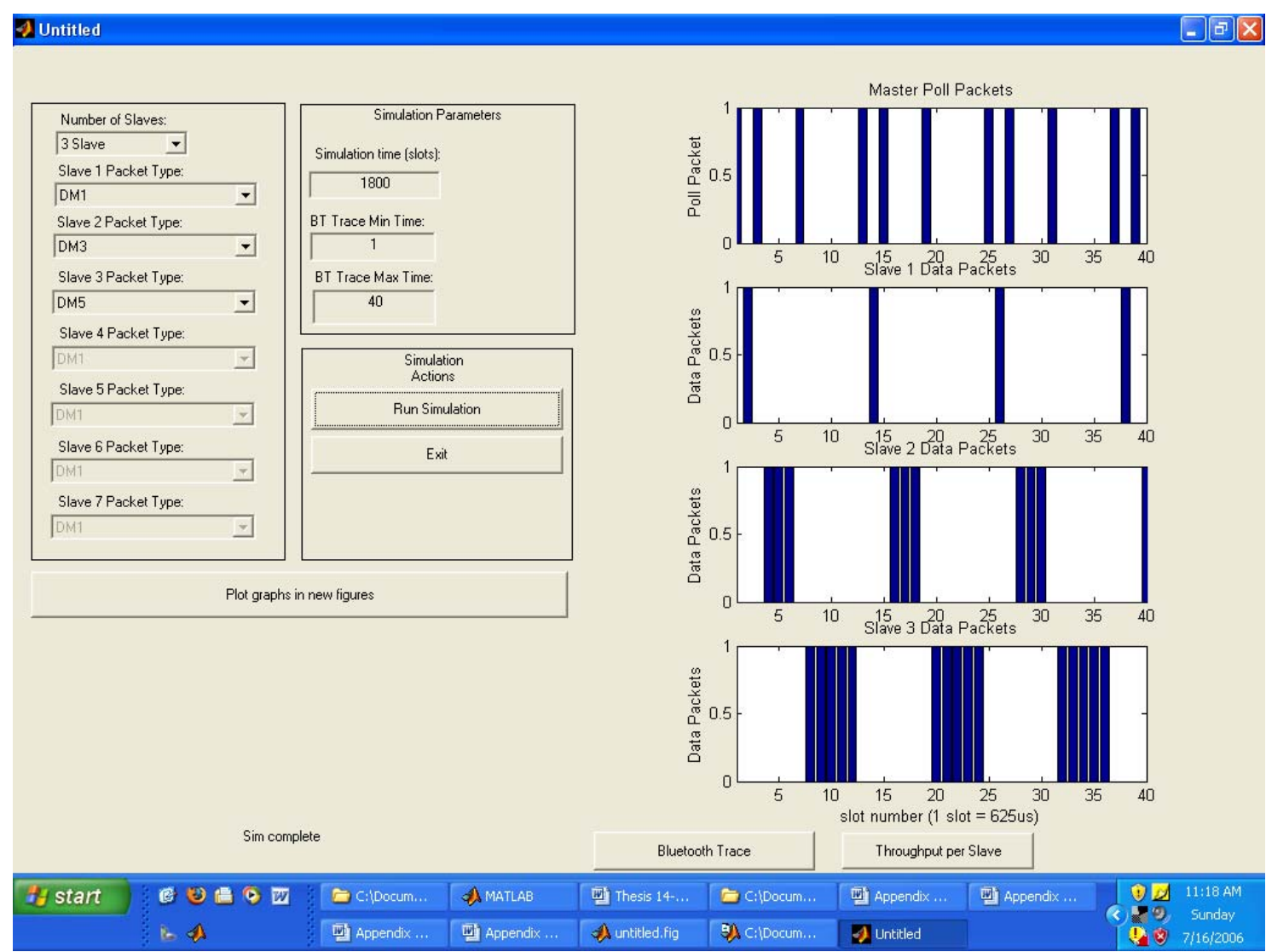




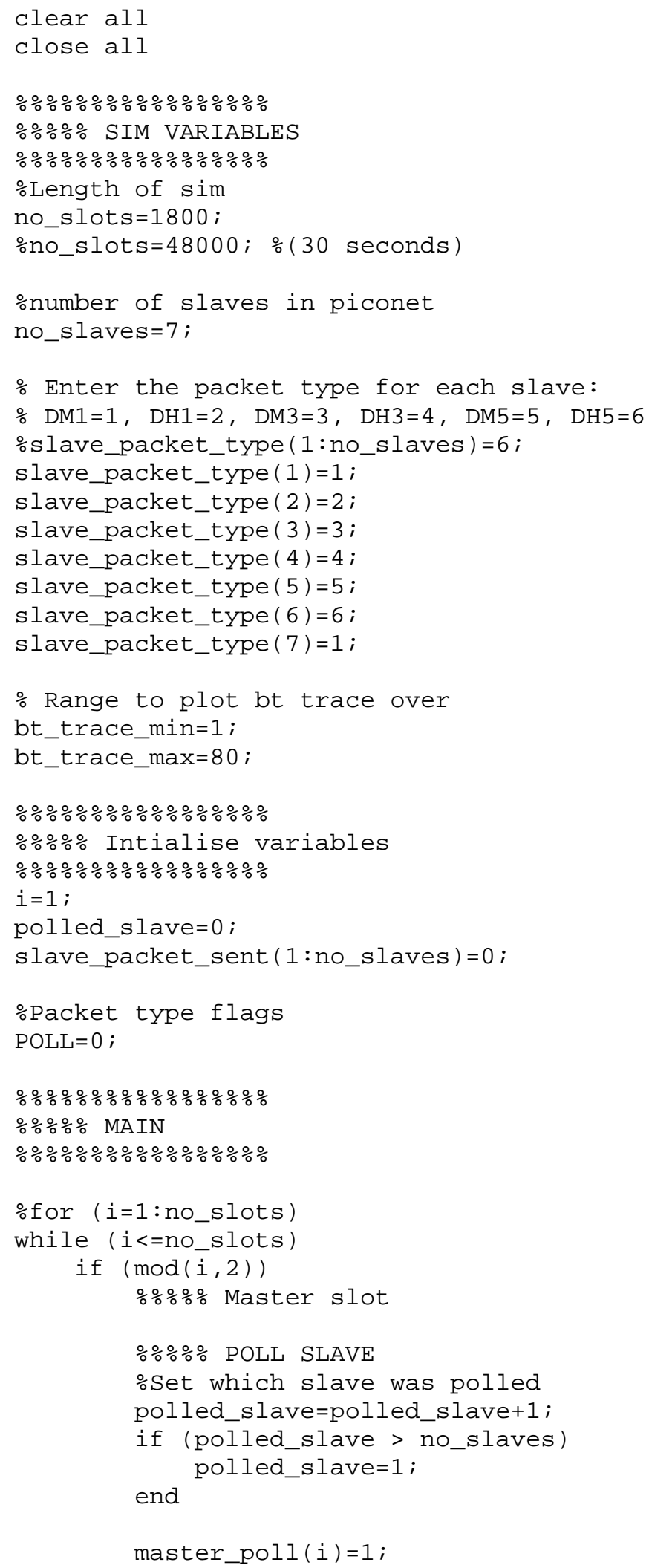




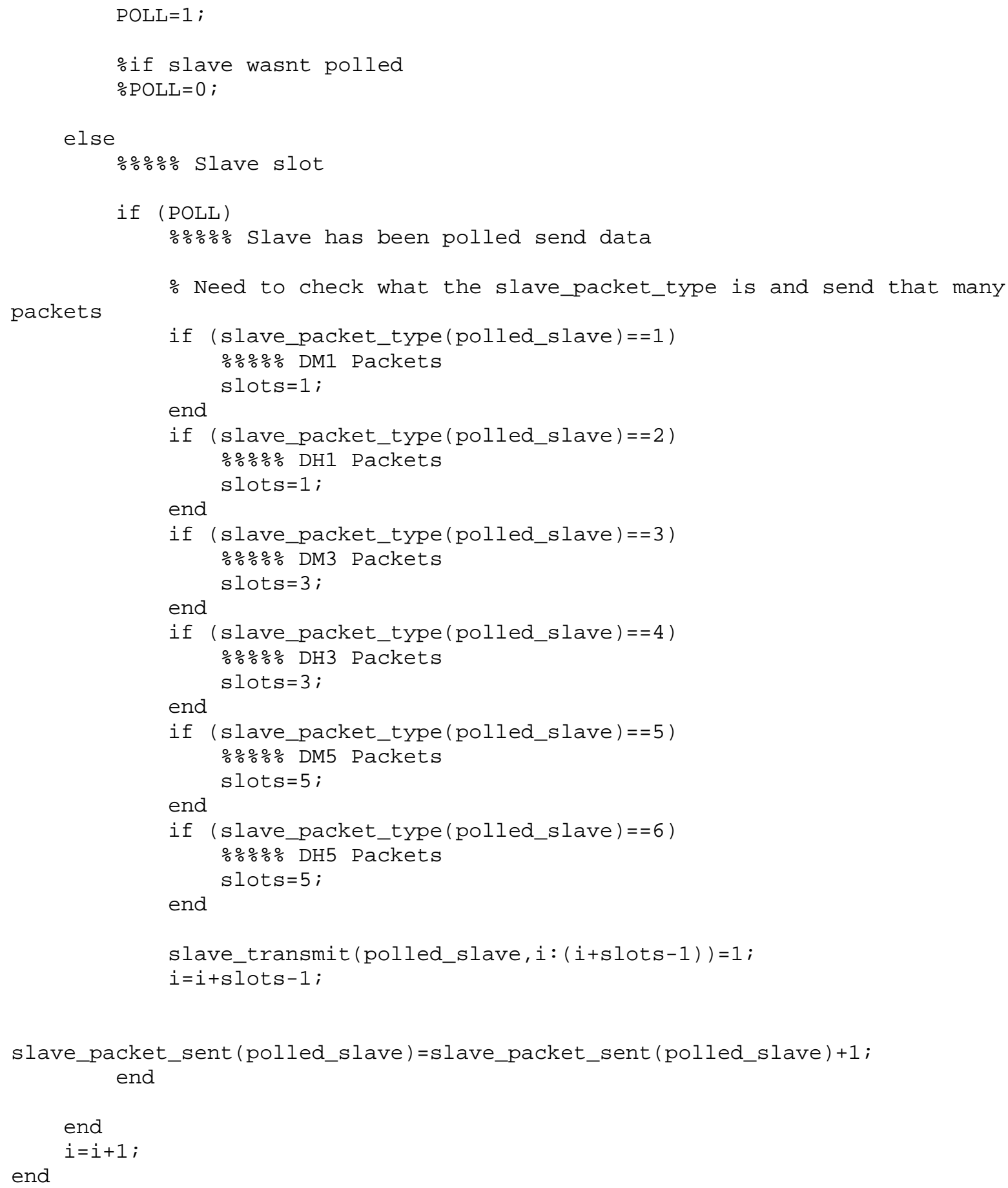




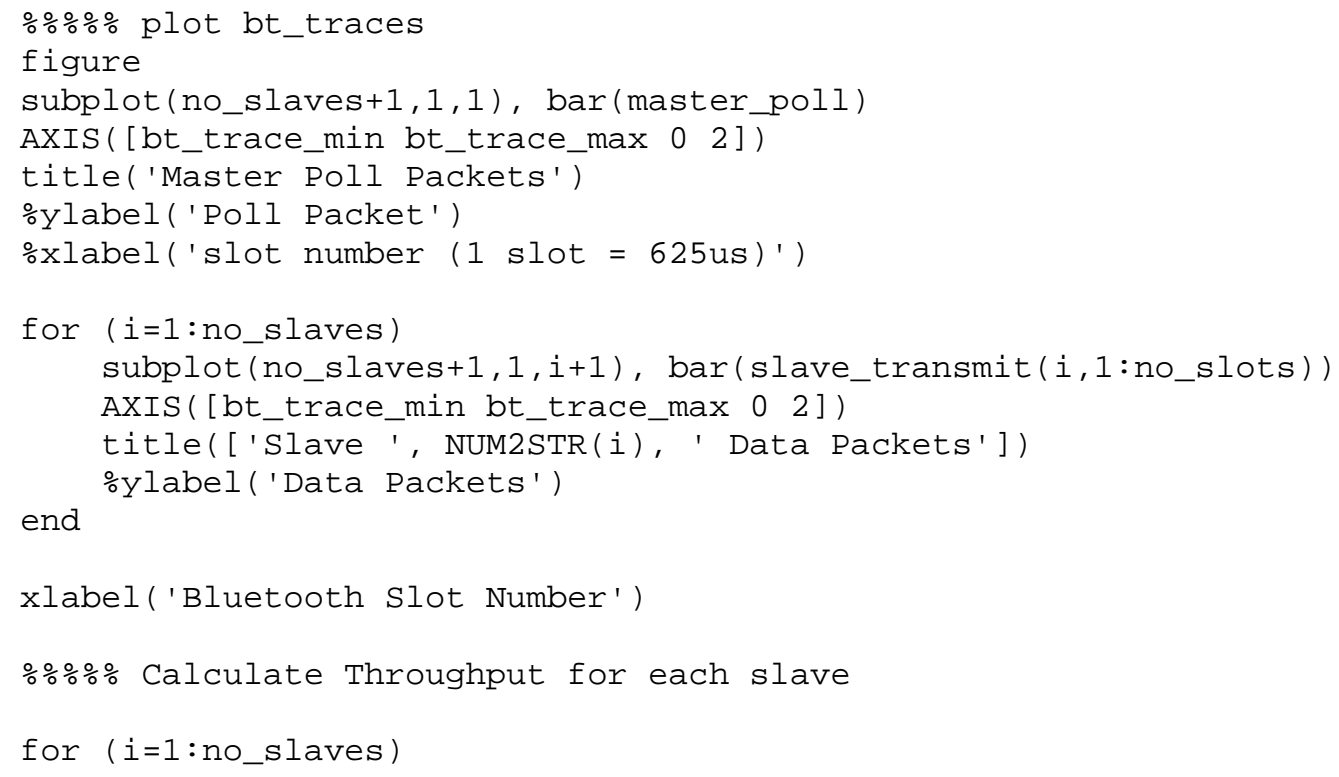


datarate

figure

\%bar (datarate/1024)

bar (datarate/1000)

grid

title('Baseband data rate throughput per slave')

ylabel('Throughput (Kbps)')

xlabel('Bluetooth Slave') 


\section{Appendix B: Bluetooth Piconet Coexistence Simulation Code}

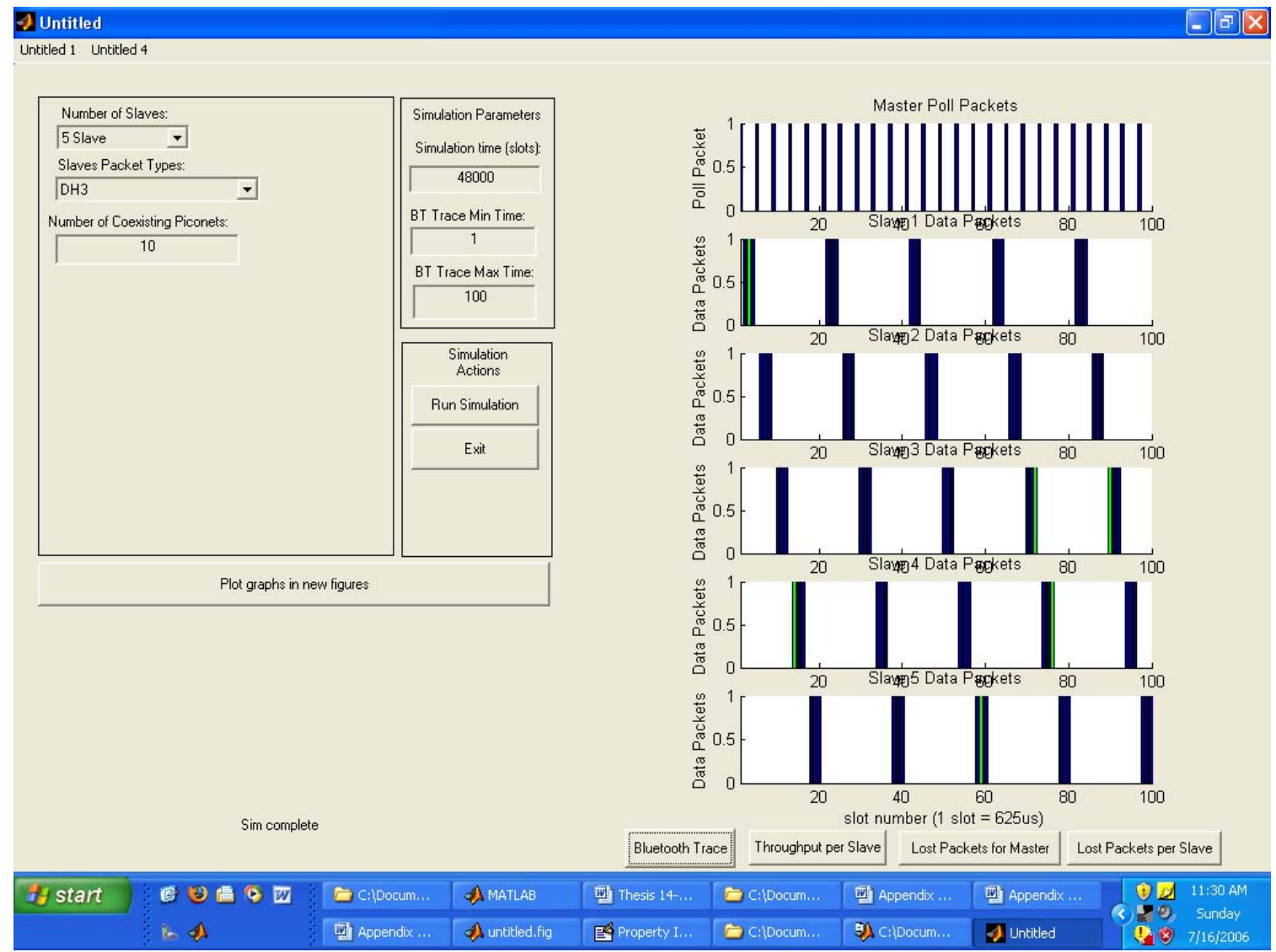


close all

\%\%\%\%\%\%\%\%\%\%\%\%\%\%

$\% \% \%$ SIM VARIABLES

\%\%\%\%\%\%\%\%\%\%\%\%\%\%

\%Length of sim

\%no_slots $=180$;

\%no_slots $=360$;

no_slots $=48000$; $\%(30$ seconds $)$

\%number of slaves in piconet

no_slaves $=4$;

\% Enter the packet type for each slave:

\% DM1=1, DH1=2, DM3=3, DH3=4, DM5=5, DH5=6

slave_packet_type $(1:$ no_slaves $)=6$;

$\% \quad$ slave_packet_type (1) $=4$;

$\%$ slave_packet_type (2) $=4$;

$\%$ slave_packet_type $(3)=6$;

$\%$ slave_packet_type $(4)=6$;

$\%$ slave_packet_type (5)=3;

$\% \%$ Coexisting Piconets

no_piconets=4;

\%\% Chance of an error for each packet (assuming the interfering piconet is sending single packets (which is the worst case))

Perror $=1-(1-1 / 79)^{\wedge}($ no_piconets -1$)$;

\% Range to plot bt trace over

bt_trace_min=1;

bt_trace_max $=80$;

\%\%\%\%\%\%\%\%\%\%\%\%\%\%\%

$\% \% \%$ Intialise variables

\%\%\%\%\%\%\%\%\%\%\%\%\%

$i=1$;

polled_slave $=0$;

slave_packet_sent $(1$ : no_slaves $)=0$;

\%Packet type flags

$\mathrm{POLL}=0$;

packet_error_slave=zeros (no_slaves,no_slots);

packet_error_master=zeros $(1$, no_slots );

lost_packets_master $=0$;

lost_packets_slaves=zeros $(1$, no_slaves $)$;

slave_transmit=zeros (no_slaves, no_slots);

master_poll=zeros(1, no_slots);

\%\%\%\%\%\%\%\%\%\%\%\%\%\%

\%\%\% MAIN

\%\%\%\%\%\%\%\%\%\%\%\%\%\%\% 


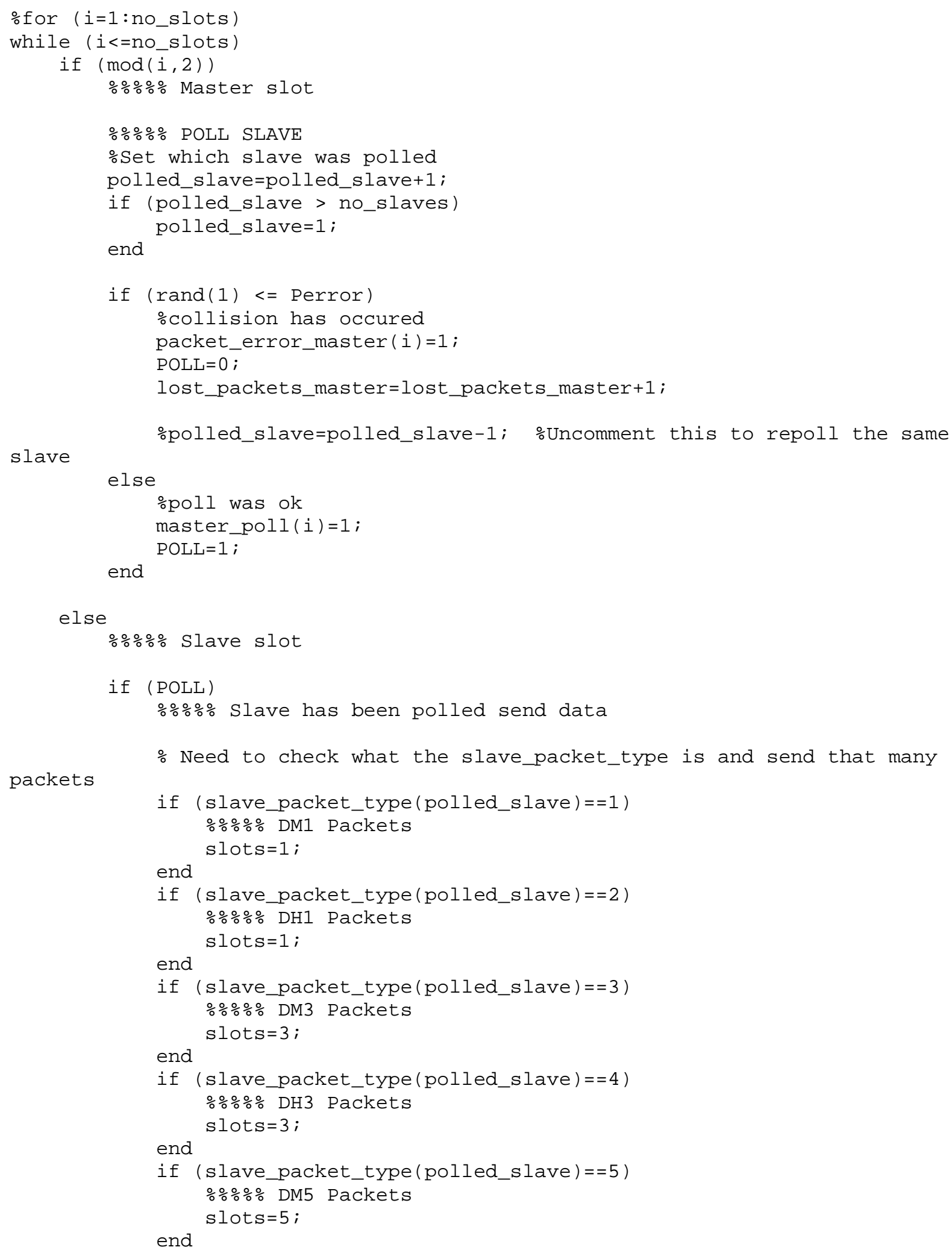




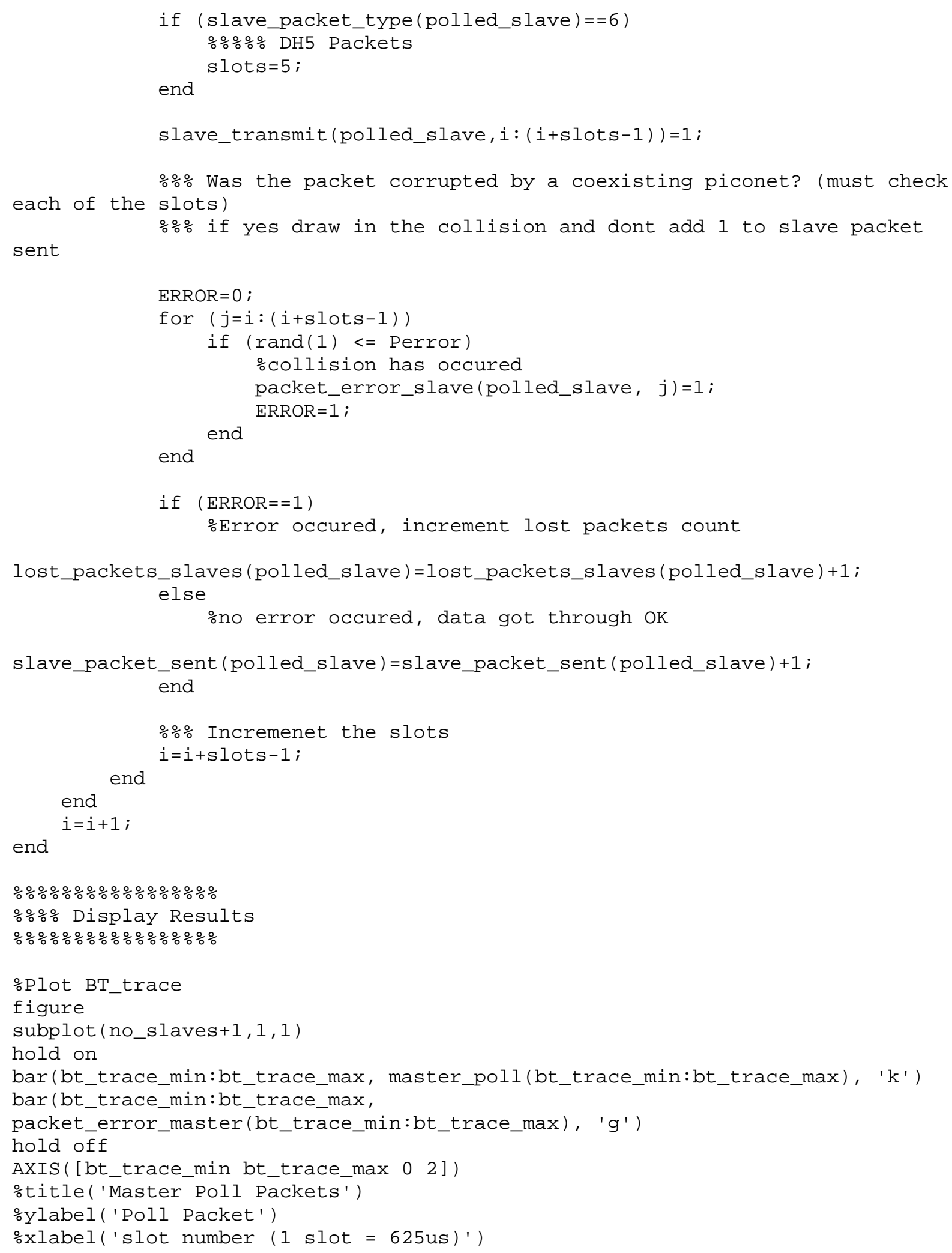




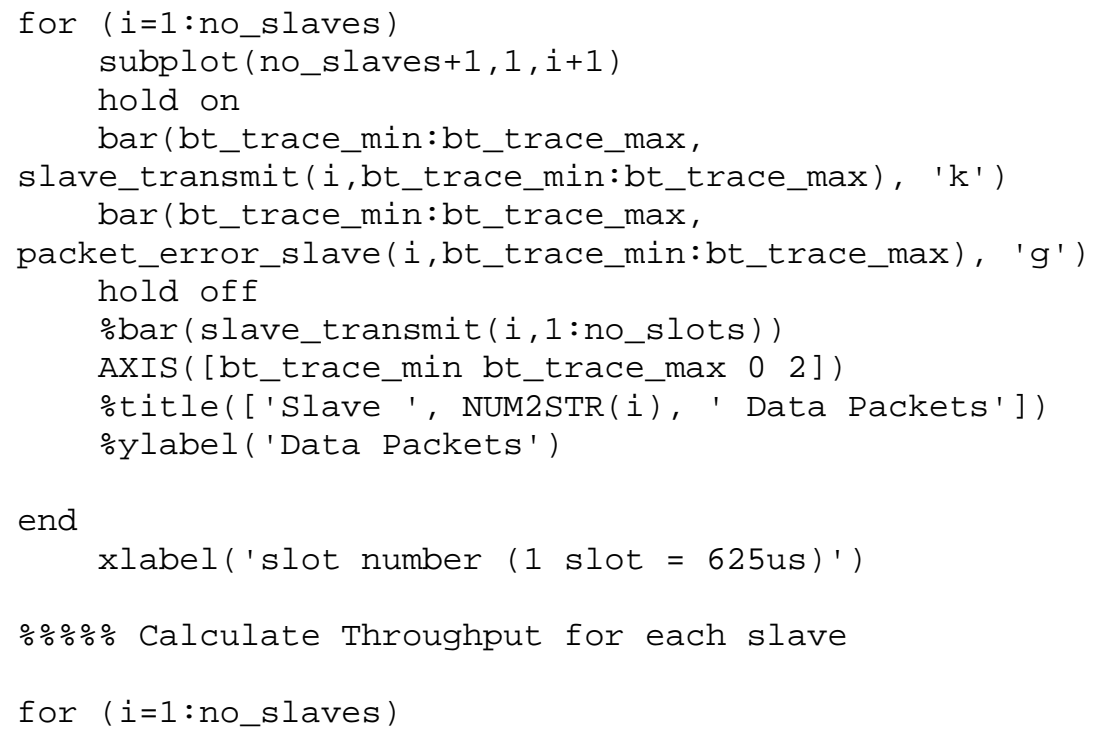

end

xlabel('slot number $(1$ slot $=625 u s)$ ')

\%\%\% Calculate Throughput for each slave

for (i=1:no_slaves)

$\%$ Need to check what the slave_packet_type is and work out datarate for that many packets sent

if (slave_packet_type(i)==1)

\%\%\% DM1 Packets (user payload 17 bytes)

end datarate $(i)=($ slave_packet_sent $(i) * 17 * 8) /($ no_slots * 0.000625$)$;

if (slave_packet_type $(i)==2$ ) $\% \% \%$ DH1 Packets (user payload 27 bytes) end 
grid

\%Plot packet errors

figure

bar(lost_packets_master)

title('Lost Packets for master')

ylabel('Lost Packets (Packets)')

xlabel('master')

figure

bar(lost_packets_slaves)

title('Lost Packets per slave')

ylabel('Lost Packets (Packets)')

xlabel('slave')

figure

hold on

bar(bt_trace_min:bt_trace_max, master_poll(bt_trace_min:bt_trace_max), 'k')

bar(bt_trace_min:bt_trace_max,

packet_error_master(bt_trace_min:bt_trace_max), 'g')

legend('Data Transmission Successful', 'Collision Occurred')

AXIS([bt_trace_min bt_trace_max+200 0 2] )

hold off 


\section{Appendix C: Piconet Sharing Bluetooth Network Structure Simulation}

\section{Code}

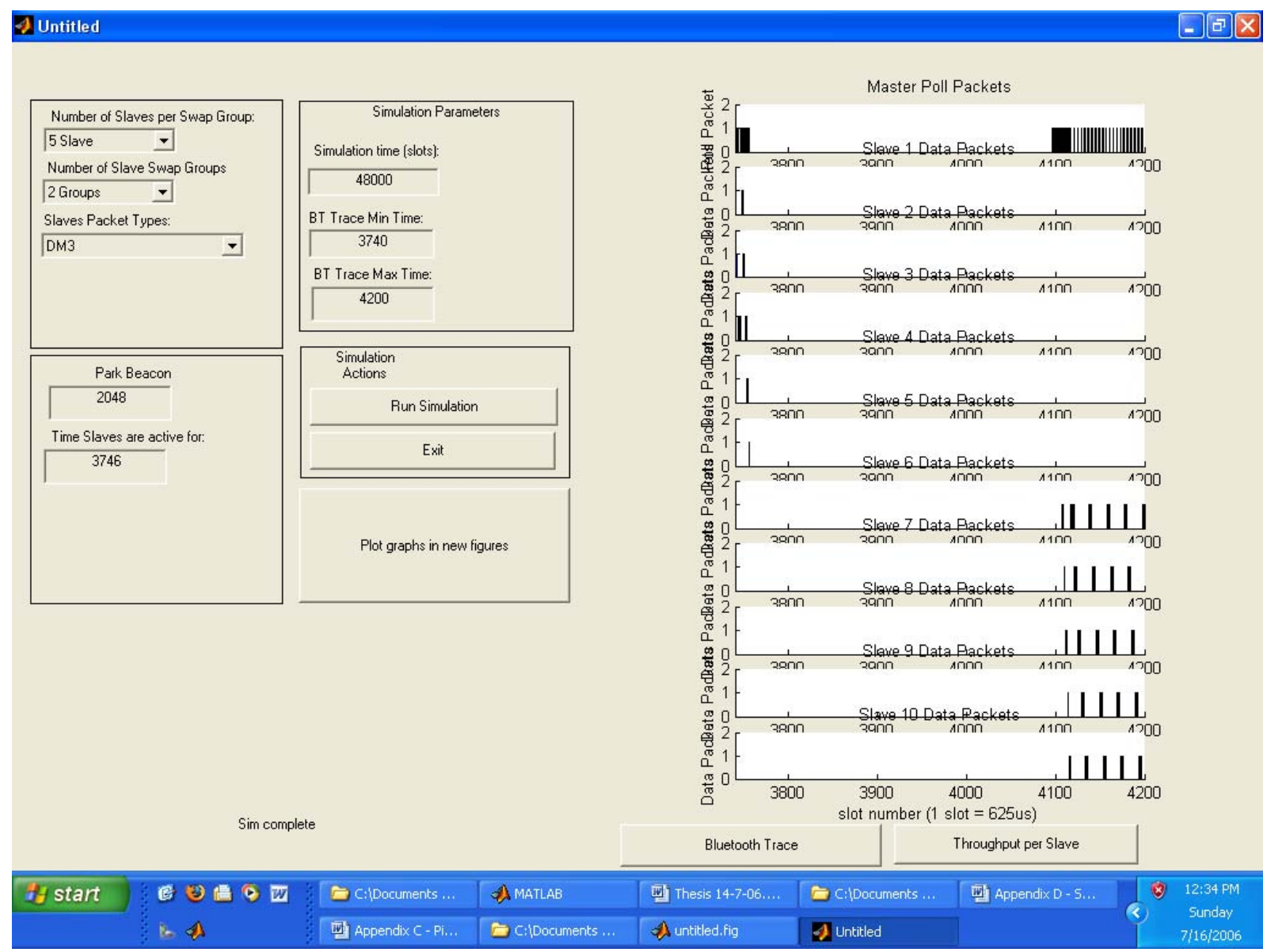


clear all

close all

\%\%\%\%\%\%\%\%\%\%\%\%\%\%

$\% \% \% \%$ SIM VARIABLES

\%\%\%\%\%\%\%\%\%\%\%\%\%\%

\%Length of sim

\%no_slots $=1800$;

\%no_slots $=48000$; $\%$ (30 seconds )

\%no_slots $=16000 ; \%(10$ seconds $)$

no_slots $=480000$; $\%$ (5 mins $)$

\%no_slots $=4800000$; \%(50 mins )

\%Park beacon

Tbeacon=2048; \%The park beacon returned by Bluemod, when parking a pan module

\%Time that slave groups are active for

active_time=3746;

\%active_time $=5000$;

\% Tpoll (Bluetooth spec defines this as maximum time between subsequent transmissions between a master and a slave)

Tpoll=50; \%This value is based on seven slaves transmiting DH5/DM5 packets, there are 43 slots between transmissions (added a bit to be sure)

\%number of slaves per swap group

no_slaves_per_group=5

\%number of groups

no_of_swap_groups=2;

\%number of slaves in piconet

no_slaves=no_slaves_per_group*no_of_swap_groups ;

\%create array of slaves

$\mathrm{s}=1$;

for ( $g=1$ : no_of_swap_groups)

for ( $h=\overline{1}:$ no_slaves_per_group)

slaves $(\mathrm{g}, \mathrm{h})=\mathrm{s}$;

end $\mathrm{s}=\mathrm{s}+1$;

end

\% Enter the packet type for each slave:

\% $\mathrm{DM} 1=1, \mathrm{DH} 1=2, \mathrm{DM} 3=3, \mathrm{DH} 3=4, \mathrm{DM} 5=5, \mathrm{DH} 5=6$

\% Data type must be same for all slaves, this is so park beacon can $\%$ easily be calculated and is always in the same place.

slave_packet_type $(1$ : no_slaves $)=6$;

\% Range to plot bt trace over

bt_trace_min $=4090$;

bt_trace_max $=4170$; 


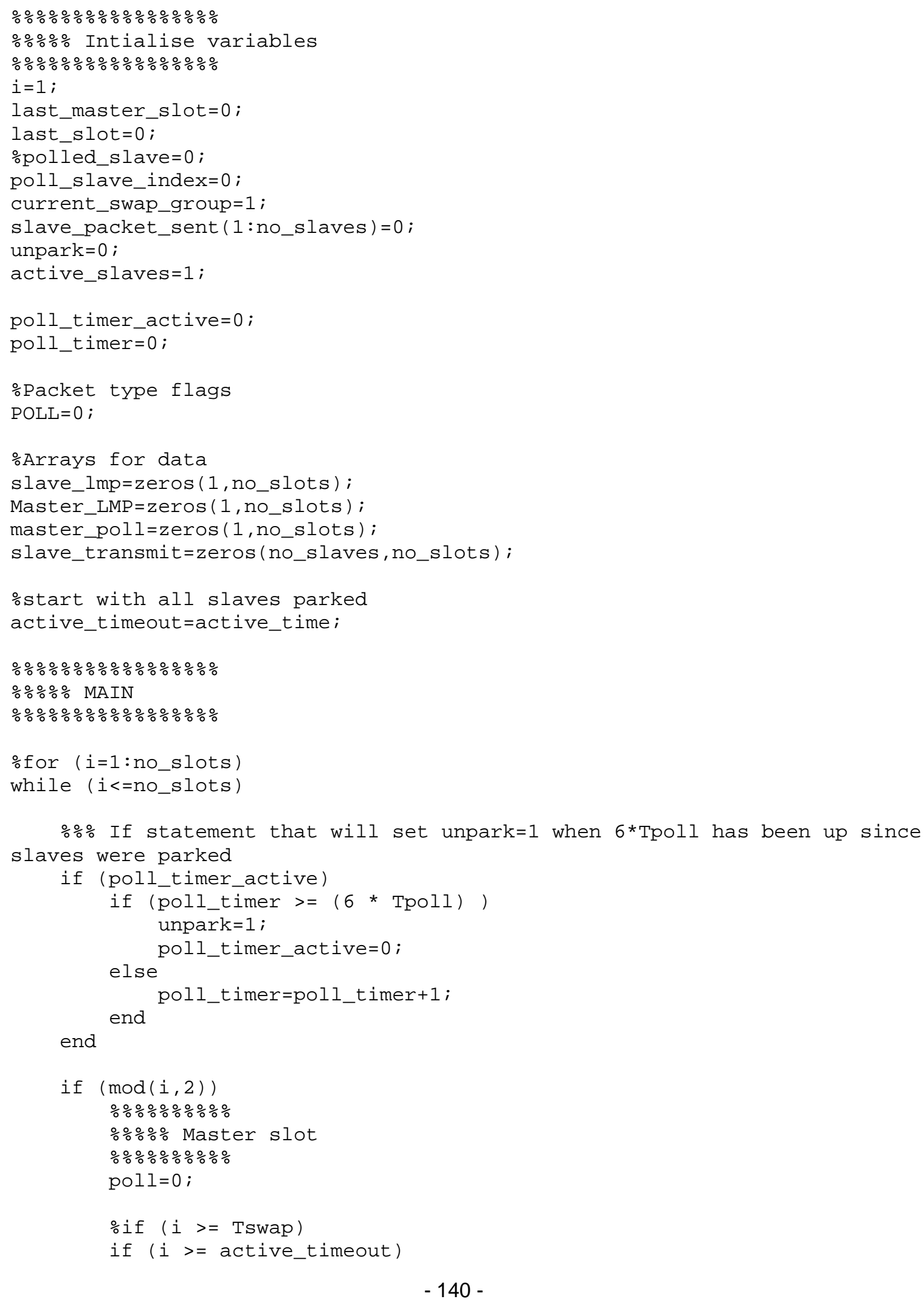




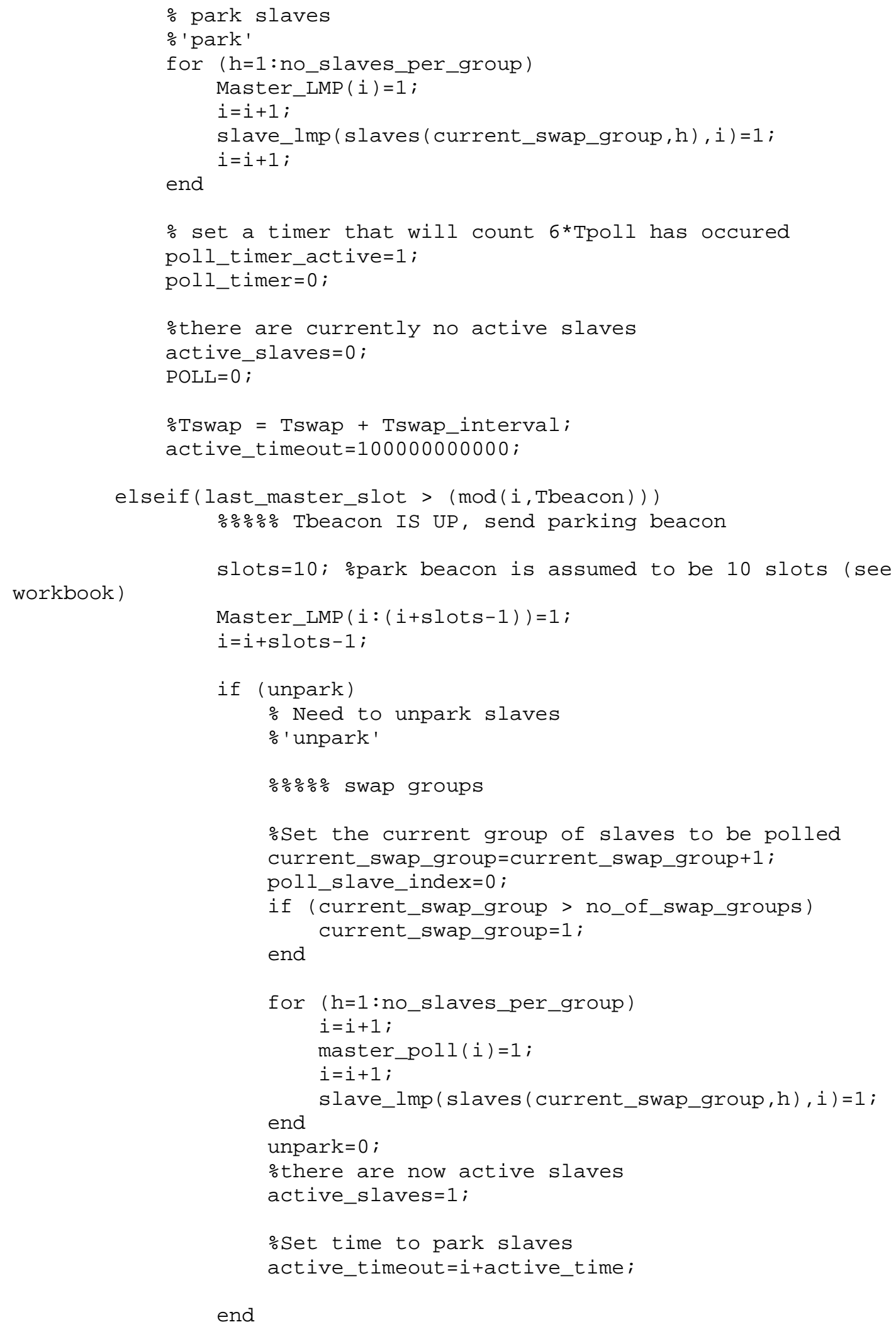




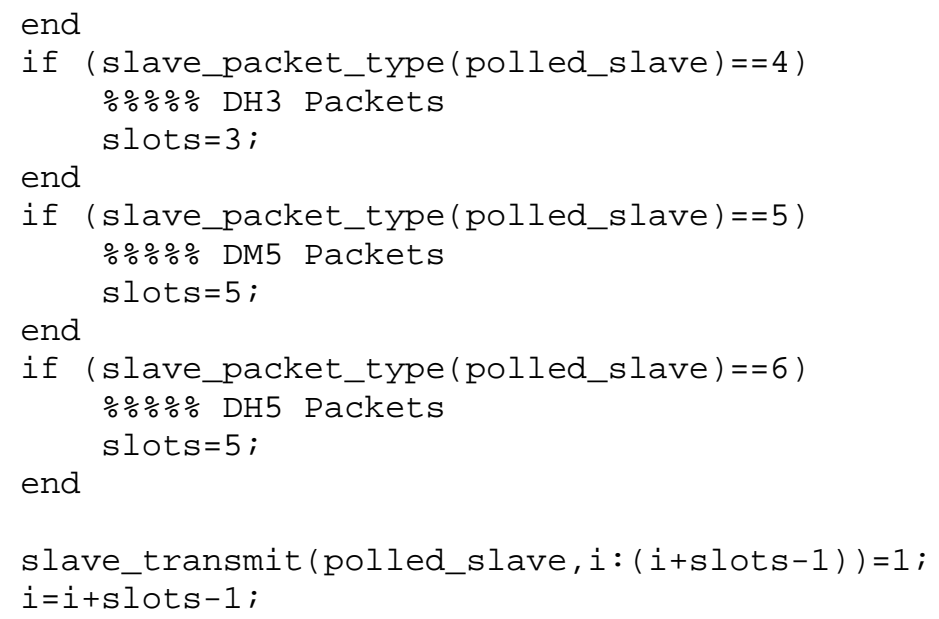




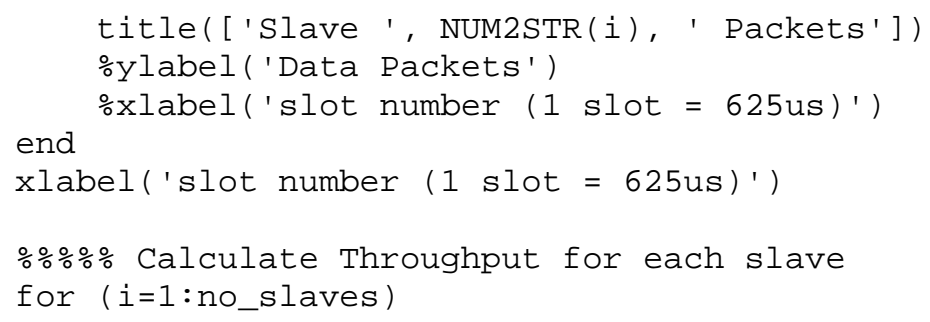


Appendix D: Scatternet Tree Bluetooth Network Structure Simulation Code

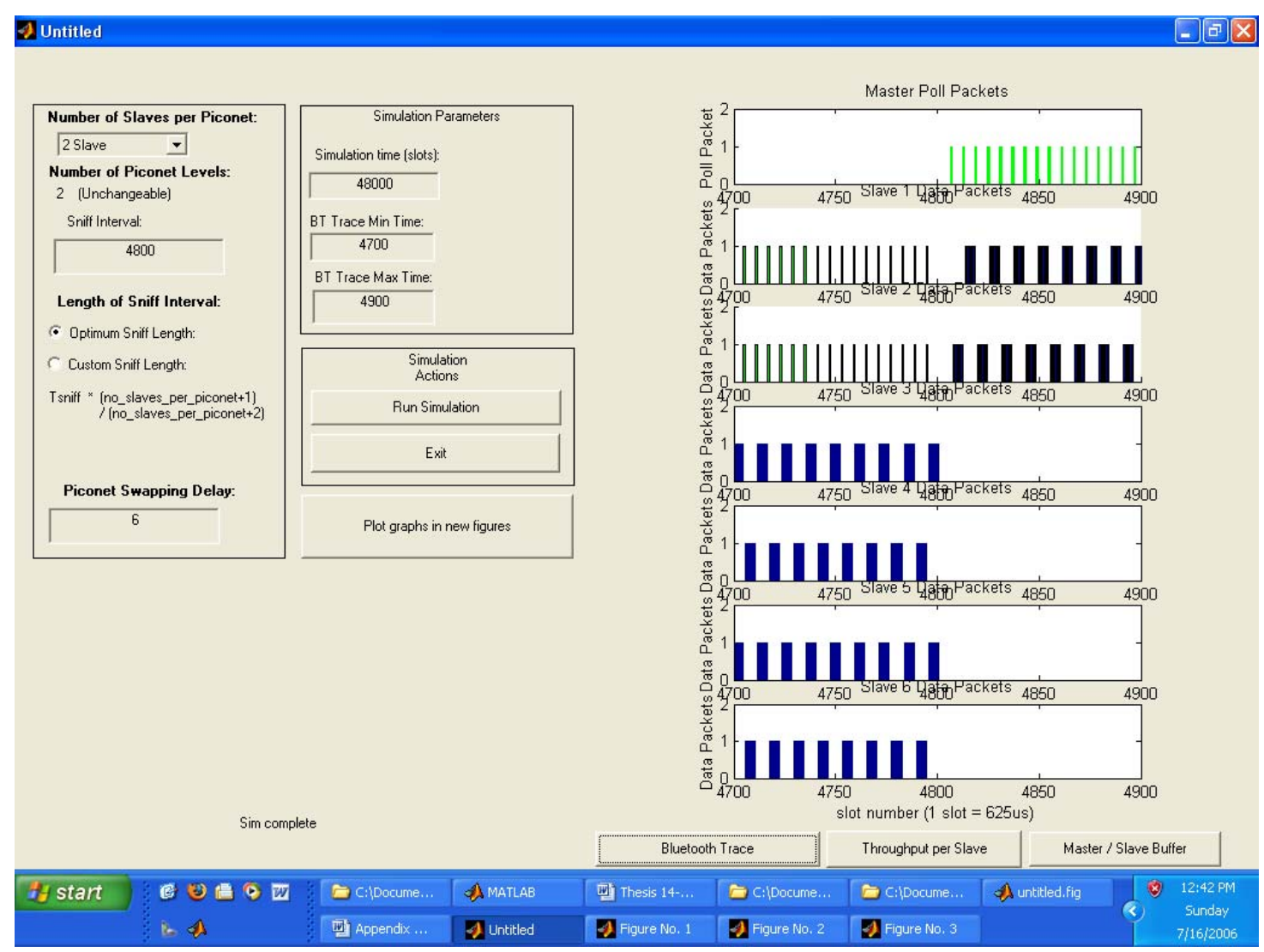


close all

\%\%\%\%\%\%\%\%\%\%\%\%\%\%

$\% \% \%$ SIM VARIABLES

\%\%\%\%\%\%\%\%\%\%\%\%\%\%

$\% \%$ Length of $\mathrm{sim}$

\%no_slots $=700$;

no_slots $=48000 ; \%(30$ seconds $)$

$\% \%$ number of slaves per piconet

no_slaves_per_piconet=3;

$\% \%$ number of piconet levels (root node is level $\odot$ )

no_of_levels=2; \%Must be set to 2 (This sim demonstrates the throughput of the M/S scatternet bridge)

$\% \%$ Tsniff Sniff interval $* * *$ Need to work out what Win CE's default settings are for this

Tsniff $=4800$;

\%Tsniff=2400;

$\% \%$ Length of sniff interval

$\%$ Nsniff_timeout=Tsniff * $1 / 2 ; \%$ as master and slave

$\%$ Nsniff_timeout=Tsniff * $3 / 4 ; \% 3 / 4$ as slave

$\%$ Nsniff_timeout=Tsniff * $4 / 5 ; \% 4 / 5$ as slave

$\%$ Nsniff_timeout=Tsniff * $5 / 6 ; \% 5 / 6$ as slave

Nsniff_timeout=Tsniff * (no_slaves_per_piconet+1) /

(no_slaves_per_piconet+2); \%This is optimum (for M/S nodes sending data)

(Master must "no_slaves_per_piconet" + 1 times longer as a slave to send the data

$\% \%$ Slot delay when swapping piconets (Must be even)

delay=6;

$\% \%$ number of nodes in scatternet (not including root node)

no_nodes $=0$;

for (i=1:no_of_levels)

end

no_nodes=no_nodes+no_slaves_per_piconet^ ${ }^{i}$;

no_nodes

$\% \%$ number of piconets in scatternet

no_piconets=1;

for (i=1: (no_of_levels-1))

end no_piconets=no_piconets+no_slaves_per_piconet^i;

$\% \%$ create array of slaves

$\mathrm{s}=1$;

for ( $g=1$ : no_piconets)

for ( $h=1$ : no_slaves_per_piconet)

slaves $(\bar{g}, h)=s$;

$\mathrm{s}=\mathrm{s}+1$; 


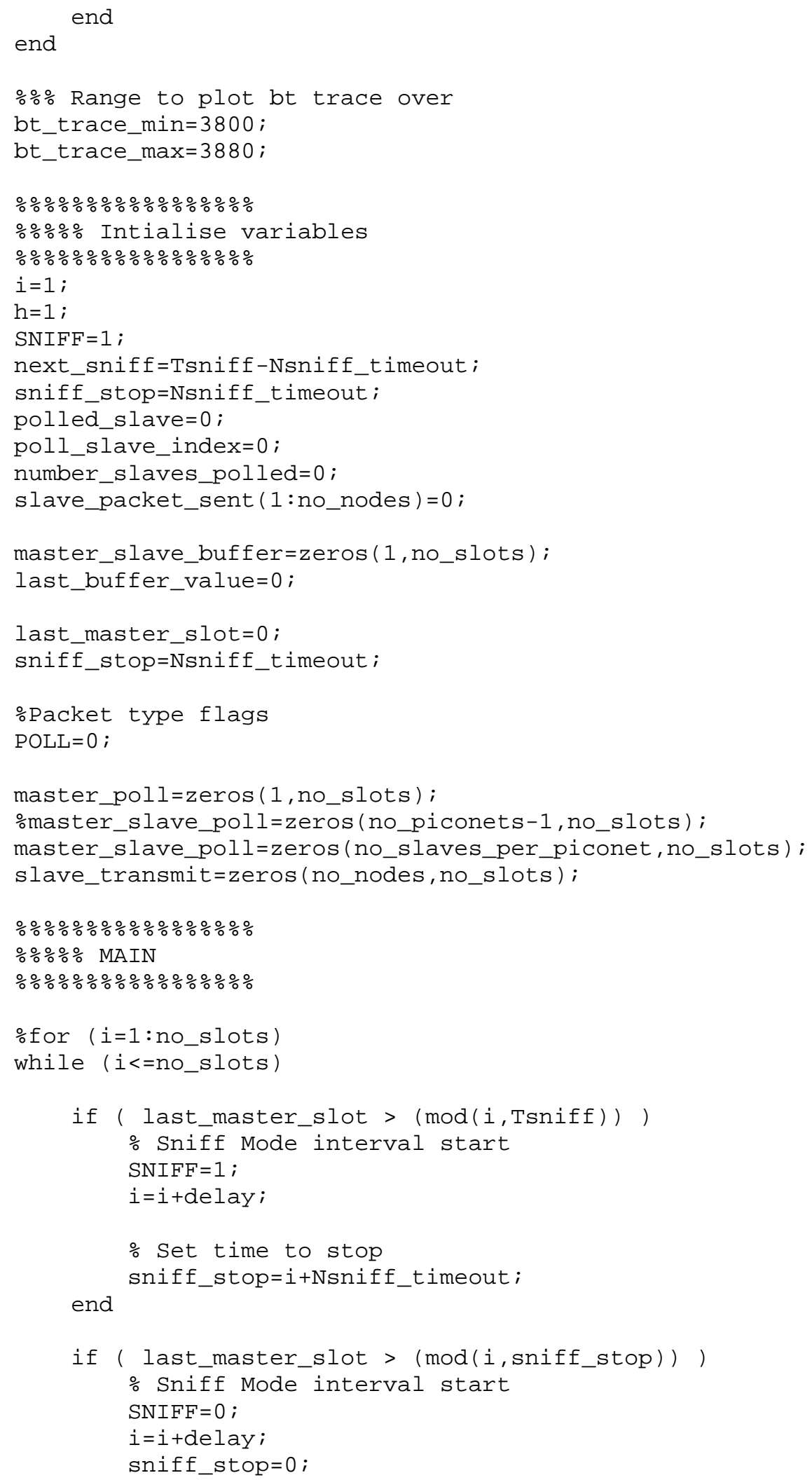


end

if $(\bmod (i, 2))$

$\% \% \%$ Master slot

if (SNIFF)

$\% \%$ Sniff Mode, Therefore M/S nodes are Slaves and Master root

node needs to poll them.

current_piconet_group=1;

$\% \% \%$ POLL SLAVE

$\%$ Set which slave was polled

poll_slave_index=poll_slave_index+1;

if (poll_slave_index > no_slaves_per_piconet) poll_slave_index $=1$;

end

\%poll slave

polled_slave=slaves(current_piconet_group, poll_slave_index); master_poll(i) $=1$;

POLL=1;

number_slaves_polled=1;

else

$\% \% \%$ Not Sniff Mode, Therefore M/S nodes are Masters and need to poll their slaves

\%Set which slave was polled

poll_slave_index $=p o l l \_s l a v e \_i n d e x+1$;

if (poll_slave_index > no_slaves_per_piconet)

end poll_slave_index $=1$;

for (current_piconet_group=2: no_piconets )

\%poll slave

polled_slave(current_piconet_group-

1) =slaves (current_piconet_group, poll_slave_index) ;

master_slave_poll(current_piconet_group-1,i)=1;

POLL=1;

end

end

number_slaves_polled=no_slaves_per_piconet ;

else

last_master_slot $=\bmod (i, T s n i f f)$;

$\% \% \%$ Slave slot

if (POLL)

$\% \% \%$ Slave has been polled send data

if (number_slaves_polled == 1)

$\% \%$ only one slave was polled (M/S nodes are slaves)

$\% \% \%$ Always DH5 Packets

slots $=5$; 
slave_transmit (polled_slave, $i:(i+s l o t s-1))=1$;

$i=i+\operatorname{slots}-1$;

slave_packet_sent $($ polled_slave $)=$ slave_packet_sent $($ polled_slave $)+1$;

$\% \% \mathrm{M} / \mathrm{S}$ node 1 has sent data, subtract this from the buffer if (polled_slave==1)

last_buffer_value=last_buffer_value-1;

if (last_buffer_value $<0$ )

\%Buffer can't send data it doesn't have

end last_buffer_value $=0$;

end

else

for (current_piconet_group=2: no_piconets)

masters) $\% \%$ More than one slave was polled (M/S nodes are

1), i: (i+slots -1$))=1$;

\section{\%\%\% Always DH5 Packets}

slots=5;

slave_transmit(polled_slave(current_piconet_group-

slave_packet_sent (polled_slave(current_piconet_group-

1) ) =slave_packet_sent (polled_slave(current_piconet_group-1) )+1;

end

$i=i+s l o t s-1$

$\% \% \% / S$ node 1 has recieved data, add this to the buffer

last_buffer_value=last_buffer_value+1;

$\% \% \% * * * \mathrm{M} / \overline{\mathrm{S}}$ node 1 generates it's own data (as often as the

slaves generate data), add this to the buffer

$\% \% \%$ This happens 1/no_slaves_in_piconet of the time

if ( $h==$ no_slaves_per_piconet)

last_buffer_value=last_buffer_value+1;

end $\mathrm{h}=\odot$;

$\mathrm{h}=\mathrm{h}+1$;

end

end

end

master_slave_buffer $(i)=$ last_buffer_value;

end $i=i+1$;

\%\%\%\%\%\%\%\%\%\%\%\%\%\%

\%\%\% Display Results

\%\%\%\%\%\%\%\%\%\%\%\%\%

\%\%\%\% plot bt_traces 
$\% \%$ Plot root node $\mathrm{BT}$ _trace

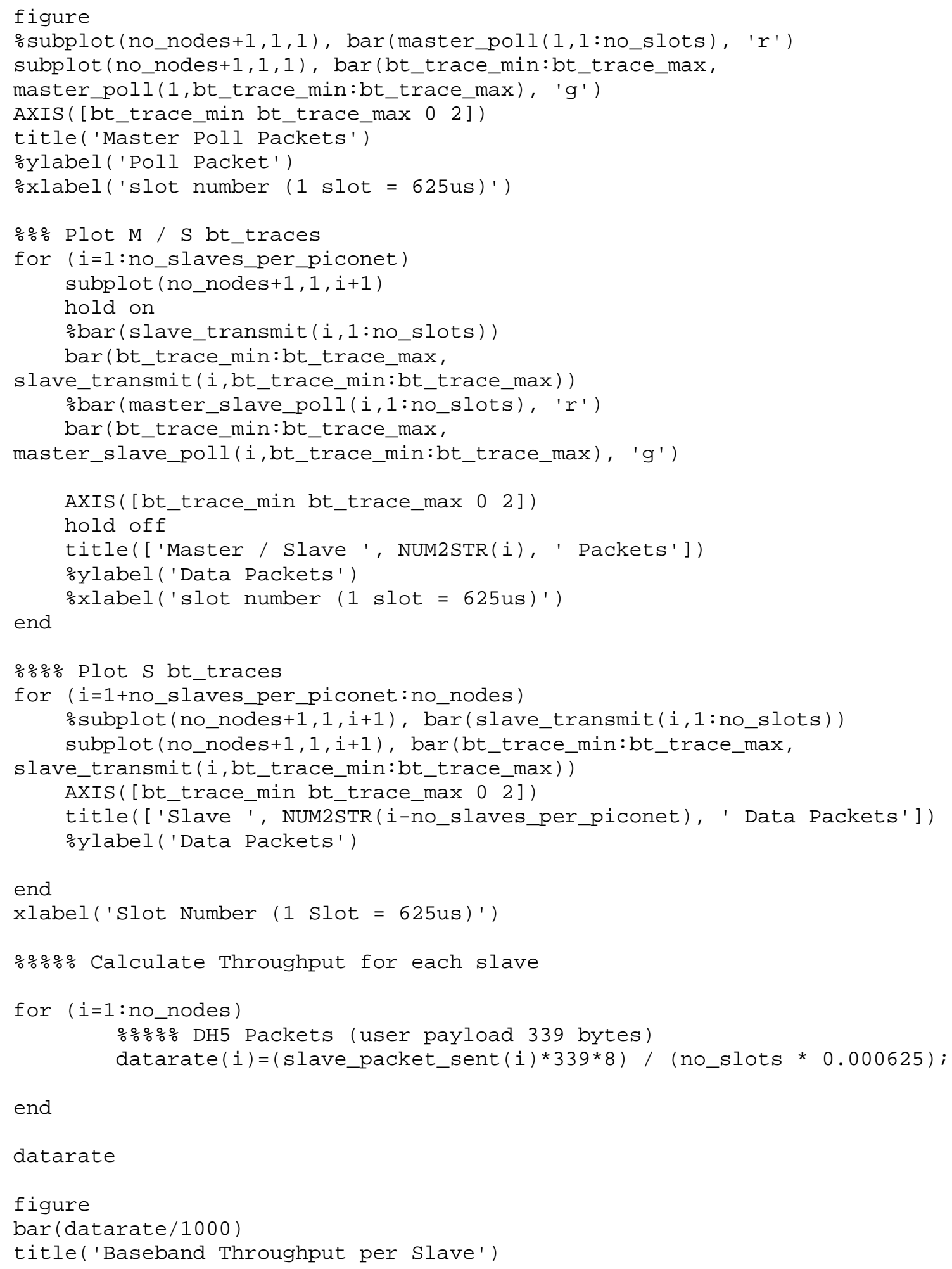

end

datarate

figure

bar (datarate/1000)

title('Baseband Throughput per Slave') 


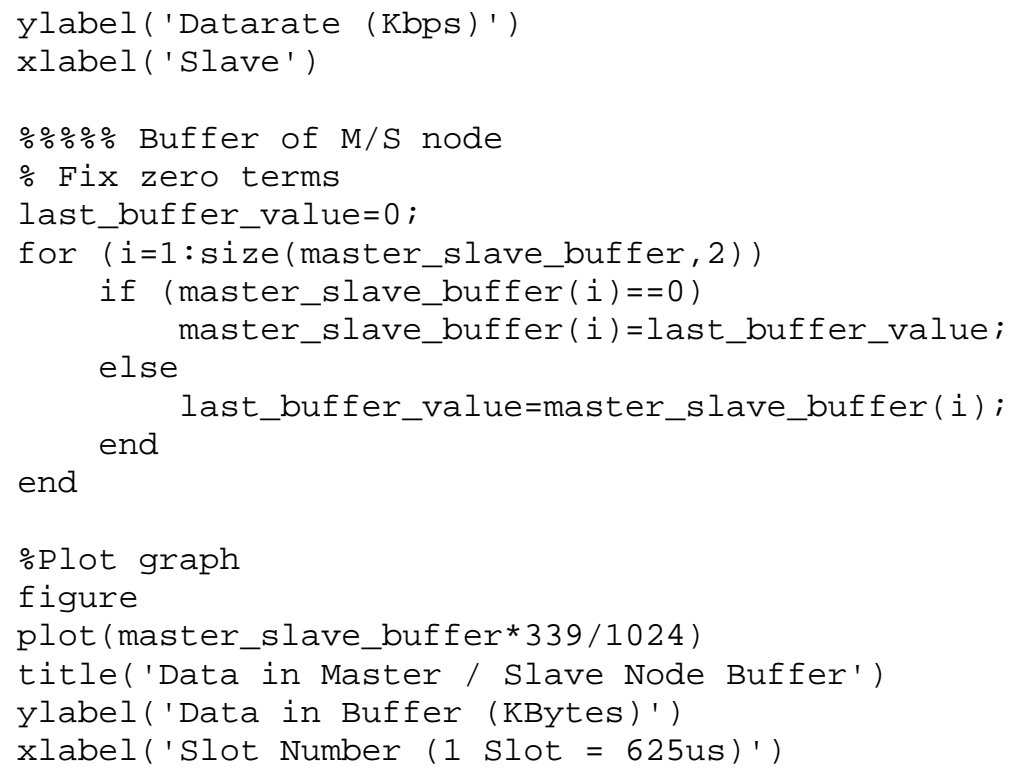




\section{Appendix E: Bluetooth RFCOMM Message Sequence Chart}

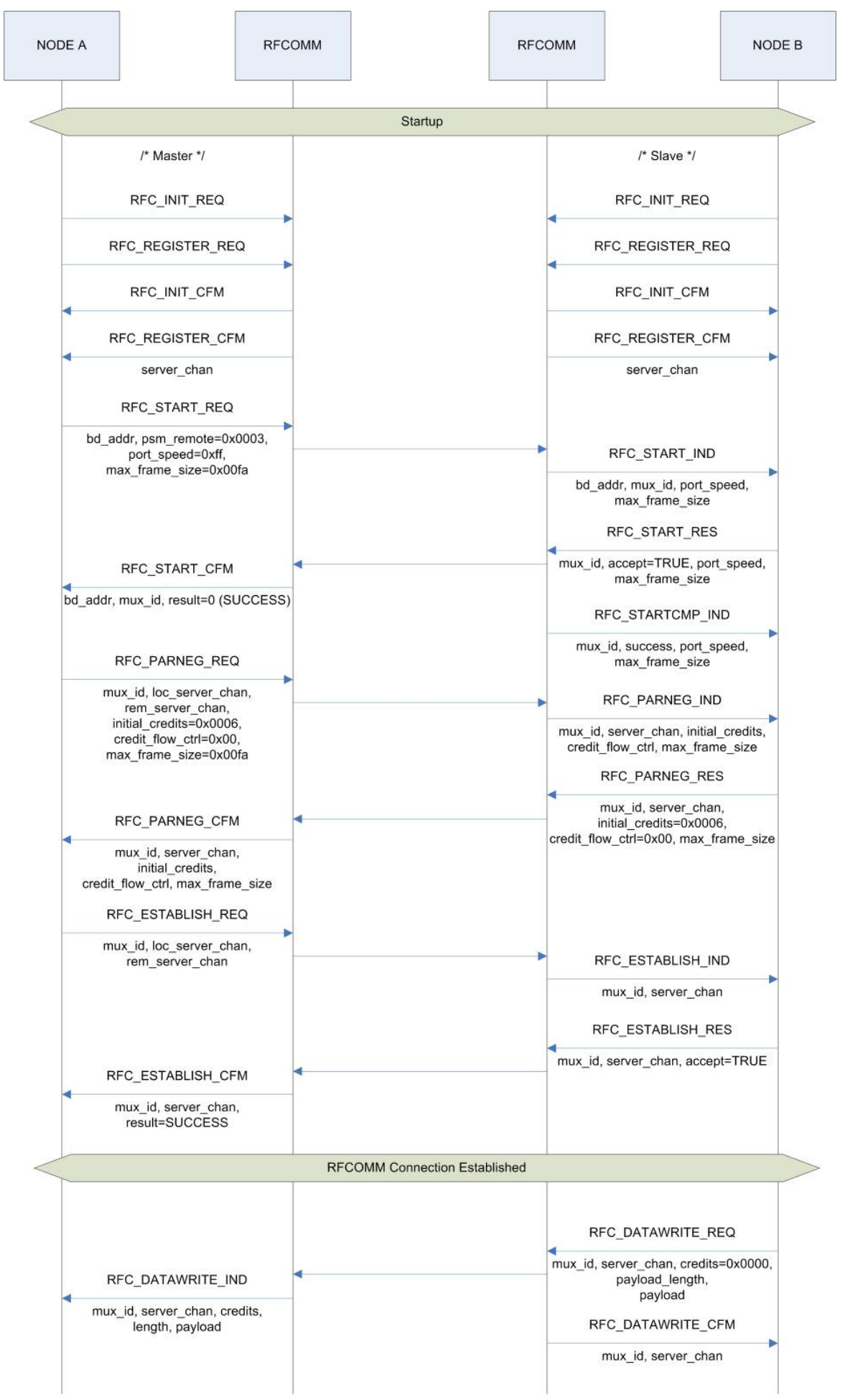




\section{Appendix F: Bluetooth Piconet Sharing Message Sequence Chart}

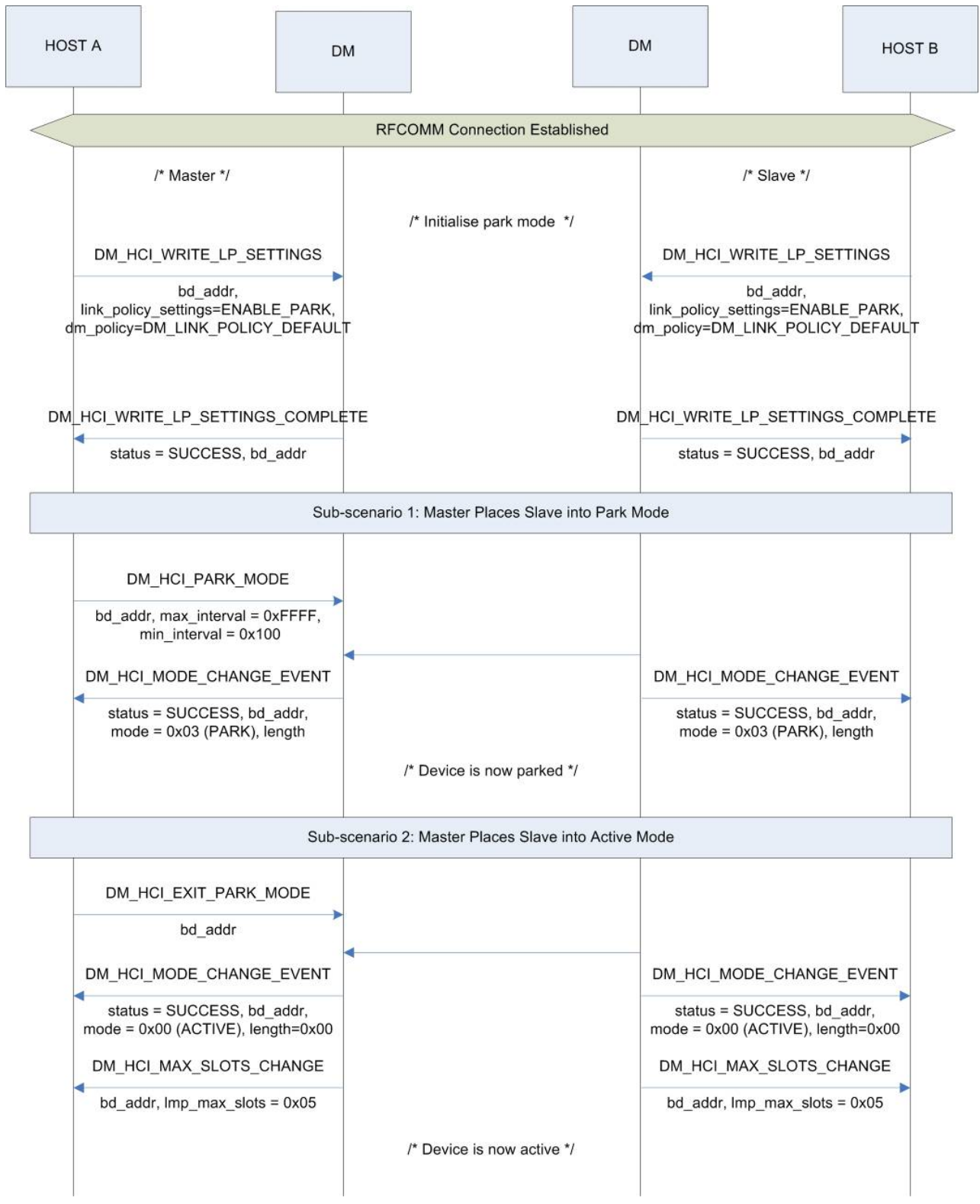




\section{Appendix G: Piconet Sharing Testing Extrapolation}

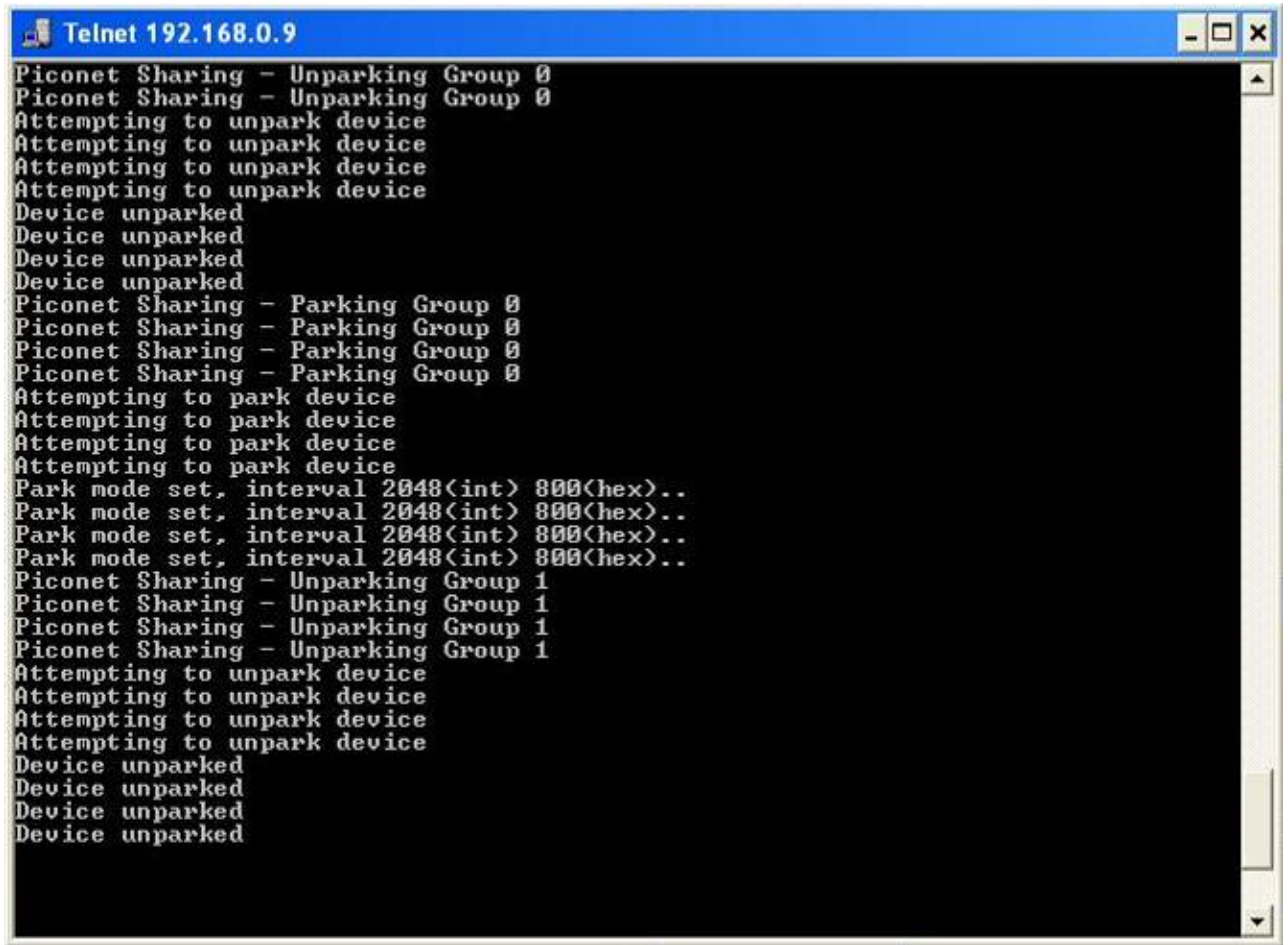




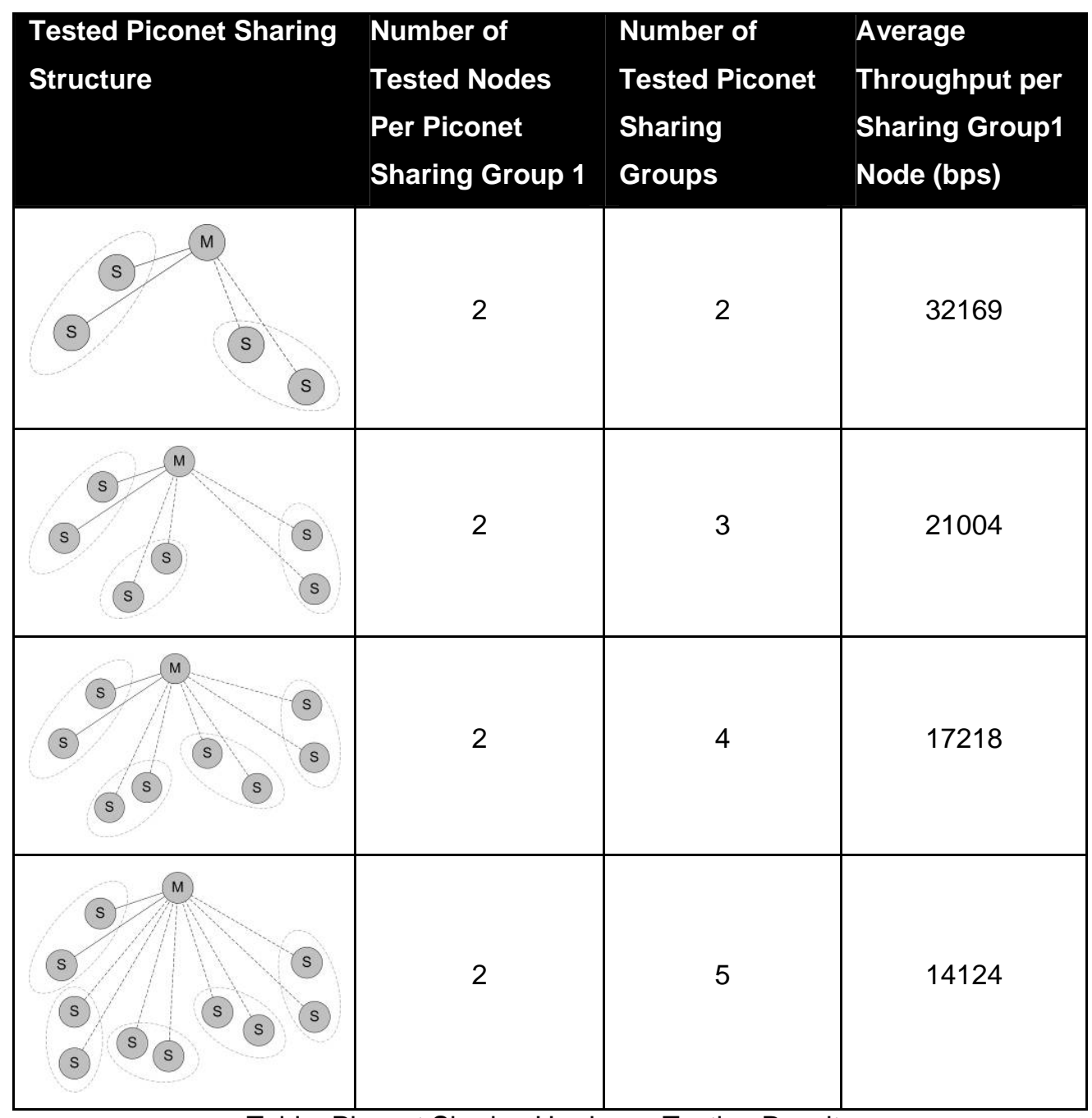

Table: Piconet Sharing Hardware Testing Results 


\begin{tabular}{|c|c|c|c|}
\hline $\begin{array}{l}\text { Tested Piconet Sharing } \\
\text { Structure }\end{array}$ & $\begin{array}{l}\text { Number of } \\
\text { Tested Nodes } \\
\text { Per Piconet } \\
\text { Sharing Group } 1\end{array}$ & $\begin{array}{l}\text { Number of } \\
\text { Tested Piconet } \\
\text { Sharing } \\
\text { Groups }\end{array}$ & $\begin{array}{l}\text { Average } \\
\text { Throughput per } \\
\text { Sharing Group1 } \\
\text { Node (bps) }\end{array}$ \\
\hline (s) & 3 & 2 & 32951 \\
\hline (s) $\int_{s}^{M} \quad$ s s & 3 & 3 & 21307 \\
\hline 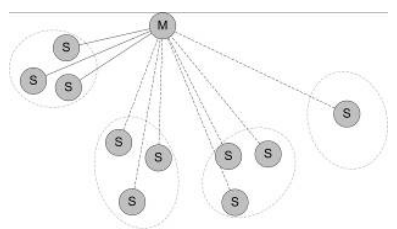 & 3 & 4 & 16107 \\
\hline
\end{tabular}

Table: Piconet Sharing Hardware Testing Results 


\begin{tabular}{|c|c|c|c|}
\hline $\begin{array}{l}\text { Tested Piconet Sharing } \\
\text { Structure }\end{array}$ & $\begin{array}{l}\text { Number of } \\
\text { Tested Nodes } \\
\text { Per Piconet } \\
\text { Sharing Group } 1\end{array}$ & $\begin{array}{l}\text { Number of } \\
\text { Tested Piconet } \\
\text { Sharing } \\
\text { Groups }\end{array}$ & $\begin{array}{l}\text { Average } \\
\text { Throughput per } \\
\text { Sharing Group1 } \\
\text { Node (bps) }\end{array}$ \\
\hline (s) & 4 & 2 & 31938 \\
\hline 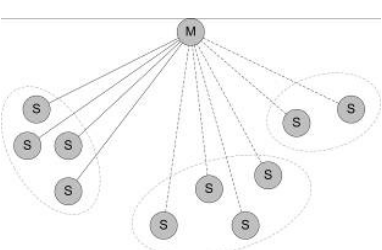 & 4 & 3 & 18120 \\
\hline 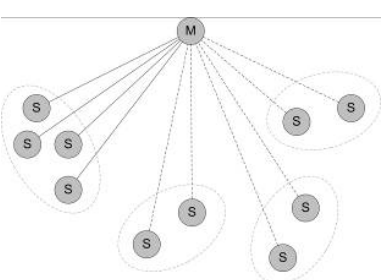 & 4 & 4 & 14547 \\
\hline
\end{tabular}

Table: Piconet Sharing Hardware Testing Results 


\begin{tabular}{|c|c|c|c|}
\hline $\begin{array}{l}\text { Tested Piconet Sharing } \\
\text { Structure }\end{array}$ & $\begin{array}{l}\text { Number of } \\
\text { Tested Nodes } \\
\text { Per Piconet } \\
\text { Sharing Group } 1\end{array}$ & $\begin{array}{l}\text { Number of } \\
\text { Tested Piconet } \\
\text { Sharing } \\
\text { Groups }\end{array}$ & $\begin{array}{l}\text { Average } \\
\text { Throughput per } \\
\text { Sharing Group1 } \\
\text { Node (bps) }\end{array}$ \\
\hline 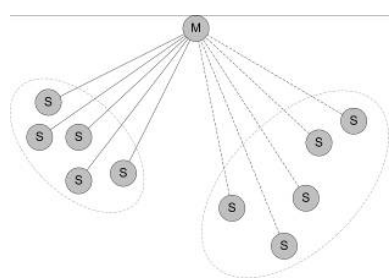 & 5 & 2 & 27320 \\
\hline $\int_{\text {(5) }}^{(3)}$ (s) $^{8}$ & 5 & 3 & 17672 \\
\hline 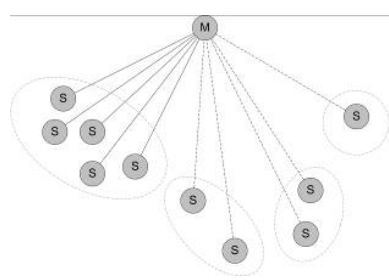 & 5 & 4 & 14041 \\
\hline
\end{tabular}

Table: Piconet Sharing Hardware Testing Results 
Appendix H: Bluetooth Scatternet Message Sequence Chart

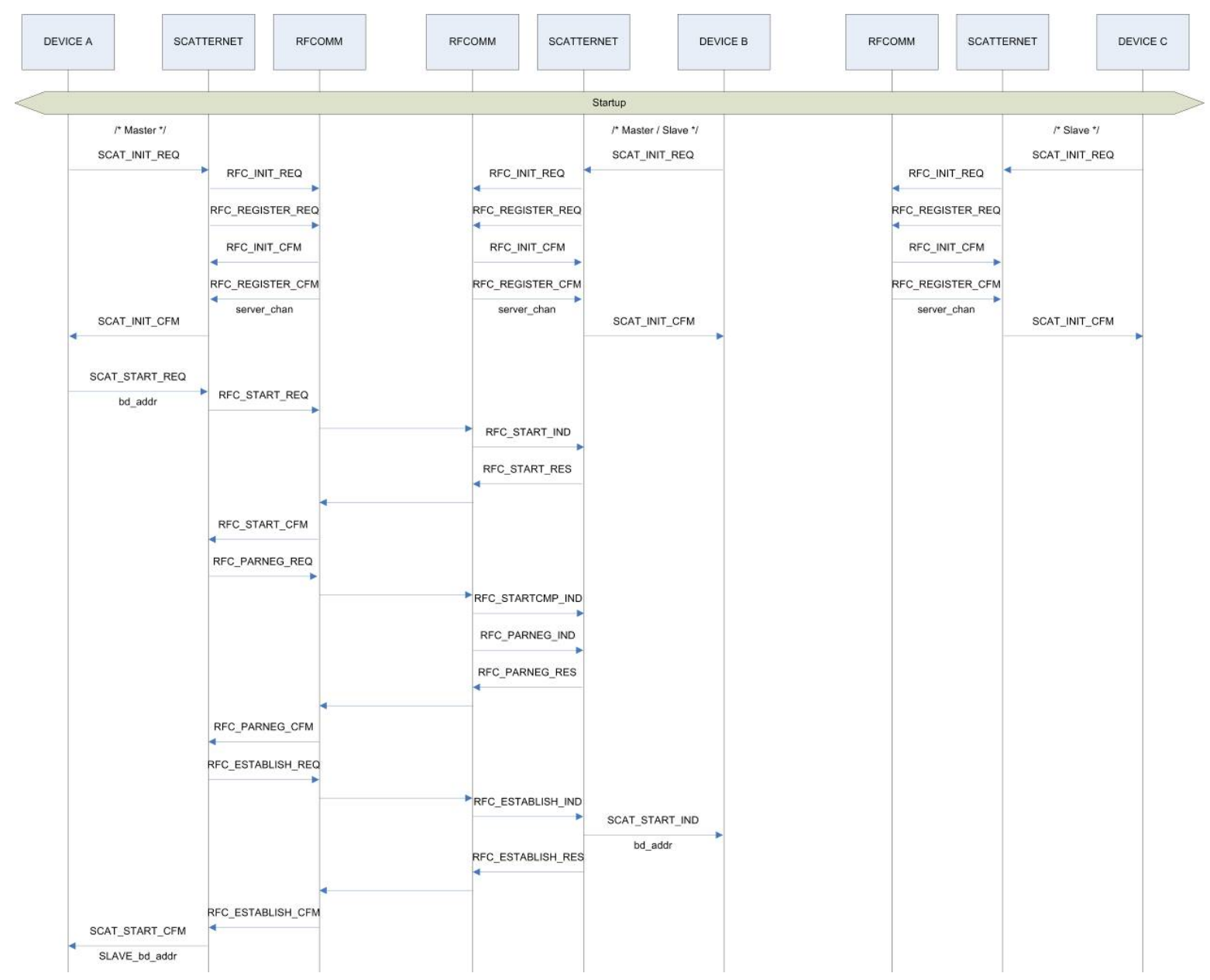




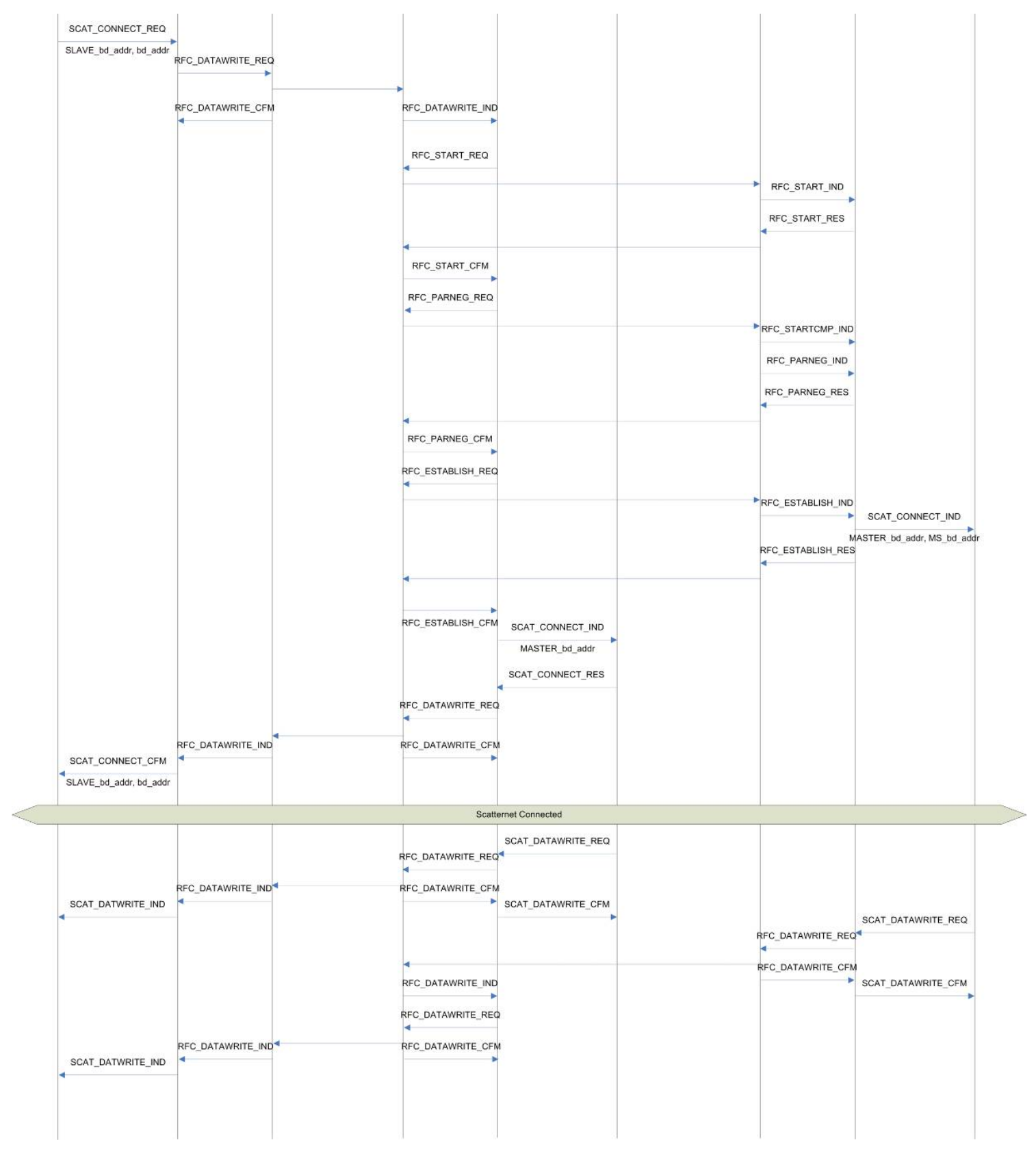

